\title{
Solid Waste Program Fiscal Year 1997 Multi-Year Work Plan WBS 1.2.1
}

J. G. Riddelle

Date Published

August 1996

Prepared for the U.S. Department of Energy Assistant Secretary for Environmental Management

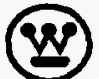

Management and Operations Contractor for the

U.S. Depertment of Energy under Contract DE-AC06-87AL10930

Approved for public release; distribution is unlimited 
LECAL DISCLANER

This report wes prepered as an eccount of work sponsored by an spency of the United States Government. Neither the United States Government nor eny agency thereof, nor eny of their employess, nor any of their contractors, subcontractors or their employeos, makes any werrenty, express or implied, or assumes any legal liability or responsibility for the aceuracy, completeness, or any third party's use or the results of auch use of any information, apparatus, product, or process disclosed, or represents that its use would not infringe privately owned rights. Reforence herein to any spocific commerciel product, process, or service by trede nemo, tredemark, memufecturer, or otherwise, does not necesserily constitute or imply its endoreement, recommendetion, or favoring by the United States Government or any egency thereof or its contrectors or subcontractors. The views and opinions of euthors expressed herein do not necessarily state or raflect those of the United Stetes Government or any agency thereof.

This report has been reproduced from the best aveilable copy. Available in paper copy and microfiche.

Aveilable to the U.S. Department of Energy and its contractors from

U.S. Department of Energy

Office of Scientific and Technical Informetion (OST)

P.O. Box 62

Oak Ridge, TN 37831

(615) 576-8401

Printed in the Urited Stetes of Ameries

DISCLM-3.CHP $\{1-91\}$ 


\section{RELEASE AUTHORIZATION}

Document Number: WHC-SP-1114, Rev. 2

Document Title: $\quad$ Solid Waste Program Fiscal Year 1997 Multi-Year Work Plan WBS 1.2.1

Release Date: $\quad 9 / 30 / 96$

This document was reviewed following the procedures described in WHC-CM-3-4 and is:

APPROVED FOR PUBLIC RELEASE

WHC Information Release Administration Specialist:

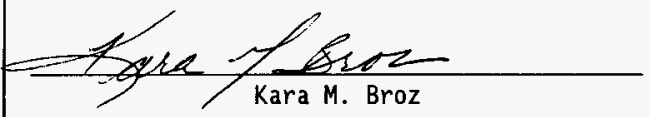

September 30, 1996 


\section{MYWP Approval Sheet \\ Solid Waste Program 1.2.1}

Mk Aenar

T. K. Teynor, Director, Waste Programs Division, Contracting Officer's Representative, DOE-RL

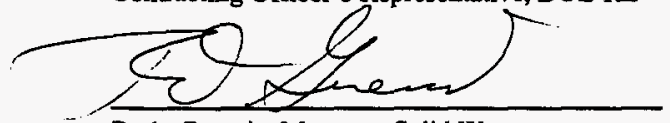

Rudy Guercia, Manager, Solid Waste

Program, DOE-RL

Whtametar fo.

W. H. Hamilton, Jr., Manager,

Solid Waste Disposal, WHC

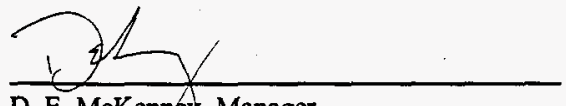

D. E. McKennex, Manager,

Restoration and Upgrade Programs, WHC

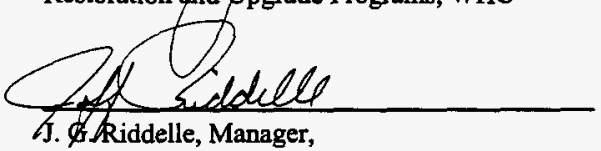

Solid Waste Programs, WHC
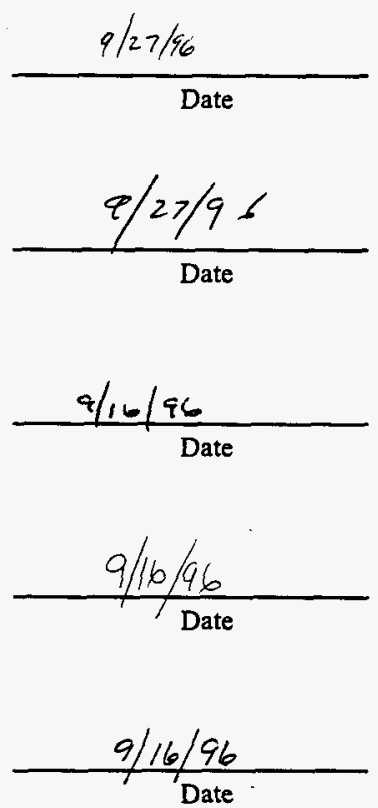

This MYWP is approved with reference to letter 96-WPD-019, Waste Programs Fiscal Year 1997 Multi-Year Work P1an (MYWP) signed on September 26, 1996, by Thomas K. Teynor, Waste Programs Division. 


\section{TABLE OF CONTENTS}

1.0 TECHNICAL BASELINE $\ldots \ldots \ldots \ldots \ldots \ldots \ldots \ldots \ldots \ldots \ldots 1-4$

1.1 Program Mission $\ldots \ldots \ldots \ldots \ldots \ldots \ldots \ldots \ldots \ldots \ldots \ldots$

1.2 Program End Point Targets $\ldots \ldots \ldots \ldots \ldots \ldots \ldots \ldots \ldots \ldots \ldots \ldots$

1.2.1 End Point Targets . . . . . . . . . . . . . . . . 1-9

1.2 .2 Technical Objectives $\ldots \ldots \ldots \ldots \ldots \ldots \ldots \ldots \ldots \ldots \ldots$

1.2 .3 Safety Objectives ..................... 1-10

1.3 Summary-Level Forecasting Data $\ldots \ldots \ldots \ldots \ldots \ldots \ldots \ldots$

1.3.1 Waste Type Data ..................... 1-10

1.3.2 Nuclear Materials . . . . . . . . . . . . . . . . 1-28

1.3 .3 Facilities $\ldots \ldots \ldots \ldots \ldots \ldots \ldots \ldots \ldots \ldots \ldots \ldots \ldots 1-28$

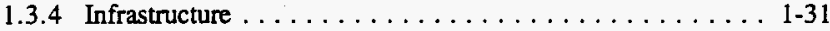

1.4 Drivers $\ldots \ldots \ldots \ldots \ldots \ldots \ldots \ldots \ldots \ldots \ldots \ldots \ldots \ldots \ldots \ldots \ldots \ldots \ldots, 34$

1.4.1 Key Drivers ....................... 1-34

1.4.2 Other Drivers . . . . . . . . . . . . . . . . . . . 1-34

1.5 Functional Definitions ..................... 1-34

1.5.1 Manage Solid Waste Remediation System . . . . . . . . . . 1-34

1.5.2 Acquire Solid Waste Remediation System ............ 1-36

1.5.3 Operate and Maintain Solid Waste Remediation System . . . . . 1-36

1.5.4 Dispose of Solid Waste Remediation System . . . . . . . . 1-37

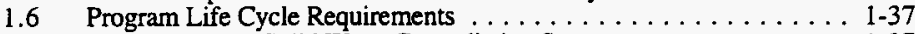

1.6.1 Manage Solid Waste Remediation System . . . . . . . . . $1-37$

1.6.2 Acquire Solid Waste Remediation System . . . . . . . . . 1-37

1.6.3 Operate and Maintain Solid Waste Remediation System . . . . . 1-37

1.6.3.1 Remedy Solid Waste Remediation System . . . . . . . 1-39

1.6.4 Dispose of Solid Waste Remediation System . . . . . . . . 1-39

1.7 Program Issues and Assumptions $\ldots \ldots \ldots \ldots \ldots \ldots \ldots \ldots, 1-40$

2.0 WORK BREAKDOWN STRUCTURE $\ldots \ldots \ldots \ldots \ldots \ldots \ldots \ldots .2-1$

2.1 Work Breakdown Structure Hierarchy $\ldots \ldots \ldots \ldots \ldots \ldots \ldots .2 .1-1$

2.2 Work Breakdown Structure Dictionary . . . . . . . . . 2.2-1

2.3 Work Breakdown Structure Responsibility Assignment Matrix . . . 2 2.3-1

3.0 SCHEDULE BASELINE $\ldots \ldots \ldots \ldots \ldots \ldots \ldots \ldots \ldots \ldots \ldots \ldots \ldots \ldots$

3.1 Program Master Baseline Schedule $\ldots \ldots \ldots \ldots \ldots \ldots \ldots \ldots$ 3.1-1

3.2 Milestone List $\ldots \ldots \ldots \ldots \ldots \ldots \ldots \ldots \ldots \ldots \ldots \ldots \ldots . .2 .1$

3.3 Milestone Description Sheets $\ldots \ldots \ldots \ldots \ldots \ldots \ldots \ldots . . \ldots \ldots .3 .1$

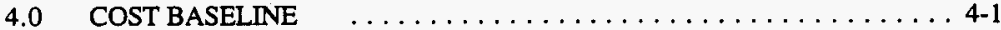

$4.1 \quad B / A$ and Cost Baseline Summaries By Year . . . . . . . . .1-1

4.2 Basis of Estimate $\ldots \ldots \ldots \ldots \ldots \ldots \ldots \ldots \ldots \ldots \ldots \ldots \ldots \ldots \ldots \ldots \ldots, 2-1$

4.3 Planned Staffing Profiles $\ldots \ldots \ldots \ldots \ldots \ldots \ldots \ldots \ldots \ldots \ldots \ldots \ldots \ldots \ldots .3 .1$ 


\section{TABLE OF CONTENTS (cont.)}

4.4 Project Work Scope Mapping . . . . . . . . . . . . . . . . 4.4-1

4.5 Cost Savings ...................... 4.5-1

5.0 EXECUTION YEAR $\ldots \ldots \ldots \ldots \ldots \ldots \ldots \ldots \ldots \ldots \ldots \ldots \ldots$

5.1 Performance Measure, Objectives, measures, and expectations . . . . 5.1-1

5.2 Program Performance Baseline Schedule . . . . . . . . . . . 5.2-1

5.3 Cost Baseline by Month . . . . . . . . . . . . . . . 5.3-1

5.4 Cost Element by Year $\ldots \ldots \ldots \ldots \ldots \ldots \ldots \ldots \ldots \ldots \ldots . \ldots \ldots . \ldots \ldots \ldots$

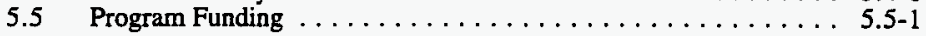

List of Tables:

1.2.1-1 End Point Targets $\ldots \ldots \ldots \ldots \ldots \ldots \ldots \ldots \ldots \ldots \ldots$

1.2.2-1 Technical Objectives . . . . . . . . . . . . . . . . . . 1-10

1.2.3-1 Safety Objectives . . . . . . . . . . . . . . . . . . 1-10

1.3.1-1 Waste Type Data . . . . . . . . . . . . . . . . . . 1-12

1.3.2-1 Nuclear Materials . . . . . . . . . . . . . . . . . . N/A

1.3.3-1 Facility Inventory . . . . . . . . . . . . . . . . . . . . 1-28

1.3.4-1 Infrastructure Requirements - Average Demand . . . . . . . . . . . . 1-32

1.3.4-2 Infrastructure Requirements - Peak Demand . . . . . . . . . . . 1-33

1.4.1-1 Key Drivers $\ldots \ldots \ldots \ldots \ldots \ldots \ldots \ldots . \ldots \ldots \ldots$ 1-34

1.5.3-1 Operate and Maintain Solid Waste

Remediation System Functions . . . . . . . . . . . . . . 1-36

1.6.3-1 Operate and Maintain Solid Waste

Remediation System . . . . . . . . . . . . . . . . . . . . . . . . 1-37

1.6.3.1-1 Remedy Solid Waste Remediation System . . . . . . . . . . . . . . . 1-39

1.7-1 Program Issues and Assumptions . . . . . . . . . . . . . 1-42 


\subsection{TECHNICAL BASELINE}

The technical baseline describes the work (functions) to be accomplished and the technical standards that govern the work. The following information is provided in this section of the MYWP: Program Mission; Program End Point Targets; Summary-Level Forecasting Data (waste type, nuclear material, facility, infrastructure needs); Drivers (key mission and regulatory): Functional Definitions; Program Life Cycle Requirements; and Program Issues and Assumptions.

\subsection{PROGRAM MISSION}

The Hanford Mission Plan, Volume 1, Site Guidance identifies the need for the Solid Waste Program to "treat, store, and dispose of a wide variety of solid material types consisting of multiple radioactive and hazardous waste classes". This includes "future Hanford Site activities which will generate new wastes that must be handled as cleanup activities are completed." Solid wastes are typically categorized as transuranic (TRU) waste, low-level waste (LLW), low-level mixed waste (LLMW), and non-radioactive hazardous waste.

To meet the mission plan need, the Solid Waste Program has defined its mission as the following receive, store, treat, decontaminate, and dispose of solid radioactive and nonradioactive dangerous wastes in a safe, cost effective and environmentally compliant manner.

As part of the Hanford Site's current mission to clean up the site, the Solid Waste Program is responsible for:

- waste located in burial grounds identified in the Low Level Burial Grounds Dangerous Waste Permit Application (burial grounds 218-E-10, 218-E-12B, 218-W-3A, 218-W$3 \mathrm{AE}, 218-\mathrm{W}-4 \mathrm{~B}, 218-\mathrm{W}-4 \mathrm{C}, 218-\mathrm{W}-5,218-\mathrm{W}-6$ [future site]), including final closure.

- $\quad$ stored solid waste mainly in buildings which are part of the Central Waste Complex, and the Transuranic Storage and Assay Facility (TRUSAF), and the Nonradioactive Dangerous Waste Storage Facility (currently in standby mode).

- managing receipts of newly generated solid waste from onsite programs and offsite generators.

- providing treatment, storage, disposal of solid waste at both onsite and offsite locations.

- storage of Shippingport Pressurized Water Reactor Core II Fuel in the T Plant canyon, and Training Reactor, Isotopes, General Atomics (TRIGA) Fuel in the active burial grounds in the 200 Areas. 
- treatment of LLMW will be principally managed through the commercial thermal treatment contract ans the commercial stabilization by macroencapsulation contract.

- WRAP 1, T Plant, and a new TPA M-33/M-91 Facility will treat the TRU waste to within acceptable standards.

- providing decontamination services for high dose waste, low dose waste, and contaminated equipment.

- managing low dose rate waste equipment for repair and return to service.

The technical functions and requirements for the Solid Waste Program are delineated in the Solid Waste Program Technical Baseline Description (WHC-SD-WM-RPT-060, current revision).

The Solid Waste Program Technical Baseline Description employs a system engineering approach to establish the program's technical baseline. The purpose of this document is to view the program as a single system and document the external and internal interfaces necessary to successfully manage a program of this magnitude. By defining the program's baseline, the effects of uncertainties and changes (internally and externally driven) to key elements of the program can be understood. Impacts of these changes to the mission and the functions of the program can be assessed and controlled.

The Solid Waste Program's mission does not include management of solid waste buried in inactive burial grounds that will be remediated by the Environmental Restoration Mission Area nor does it include CERCLA waste generated on site by the Environmental Restoration contractor. However, there will be some waste generated by the ER contractor that is within the scope of the mission (I.e., it will be shipped to the Solid Waste Program as newly generated waste).

The following paragraphs describe the type of waste, decontamination services, and the storage of Reactor Irradiated Nuclear Material (RNM).

\section{Transuranic (TRU) Waste}

Management of all retrieved, stored, and newly generated TRU (and TRU mixed) waste shall be the responsibility of the Solid Waste Program. The program is responsible for retrieving waste placed in retrievable storage since 1970 (located in 218-W-3A, $-4 \mathrm{~B},-4 \mathrm{C}$, and 218-E-12B Burial Grounds). The program is also responsible for providing onsite TRU waste storage until an offsite disposal system is ready to accept Hanford Site material. The program is to provide for treatment and certification of TRU waste to meet waste acceptance criteria for disposal. The program is also responsible for arranging for transportation of the TRU waste prepared for 
offsite disposal at the Waste Isolation Pilot Plant (WIPP). Treatment, certification, and preparation for transport to WIPP of TRU waste will be performed at WRAP, T Plant, and a new TPA M-33/M-91 facility.

\section{Low-Level Mixed Waste}

The Solid Waste Program is responsible for the receipt, treatment, interim storage, and disposal of low-level mixed waste assigned to the Hanford Site for disposition. The Hallam sodium will continue to be maintained by the program during FY1997. The program provides systems (include tracking databases, waste configuration and permit maintenance) to assure that waste is regulatorily acceptable. Where offsite treatment is required to allow disposal of the waste, program responsibility includes onsite storage and arranging for transport to the offsite treatment location. Final disposition will occur in a RCRA permitted onsite disposal system. Program responsibility includes closure and post closure maintenance of the disposal system.

\section{Low-Level Waste}

The Solid Waste Program is responsible for the receipt and disposal of multiple categories of low-level waste assigned to the Hanford Site for disposition. Final disposition will occur in an onsite disposal system. Program responsibility includes closure of the disposal system. It is not the intent of the program to "store" LLW except in special cases.

\section{Hazardous Waste}

The Solid Waste Program provides for disposition of hazardous wastes generated by onsite programs. The program is responsible for arranging for transportation of hazardous waste to an offsite treatment, storage or disposal site. Currently the 616 building is in standby condition, and is available for interim storage of hazardous waste should it be needed.

\section{Decontamination Services}

The Solid Waste Program provides decontamination services of high dose rate waste and contaminated equipment to meet applicable standards for disposal, storage, re-use, or free release. The Solid Waste Program also performs decontamination, waste verification/characterization, and repackaging of incoming wastes for reduction in radiation fields and recategorization of wastes from mixed to non-mixed and/or Greater Than Category 3 low-level to Category 1 or 3 low-level or, if possible, to levels making the waste a releasable (nonradioactive) material. Low dose rate waste contaminated equipment is also managed for repair and return to service. 


\section{Storage of Reactor Irradiated Nuclear Material (RINM)}

The Solid Waste Program through the T Plant canyon provides storage for RINM, namely the Shippingport Pressurized Water Reactor Core II (DOE 1995). In addition a small amount of Training Reactor, Isotopes, General Atomics (TRIGA) fuel in the active burial grounds in the 200 Areas has been classified as RINM and is to be managed as such (DOE 1995). The disposition of this RINM was addressed in the DOE programmatic SNF Fuel Management and INEL ER and WM programs FEIS. The Solid Waste Program may provide functions required to implement the record of decision of this EIS. In the interim, the Solid Waste Program will continue its existing storage management of the RINM and may complete interim actions to continue safe management. As yet, no definitive date $h$ as been set for removal of the TRIGA fuel from its current storage in the burial grounds.

\section{Canister Storage Building}

The canister storage building will be maintained by the Solid Waste Program at the conclusion of the SNF Project, currently scheduled for FY2001.

\section{Closure of LLW Burial Grounds}

The LLW Burial Grounds will be closed in accordance with the applicable RCRA guidelines and Performance Assessment (PA).

\section{Programmatic Assumptions}

- The WRAP TRU schedule reflecting TRU line startup on 10/1/97 and WIPP shipment on 1/1/98 is not fully funded. Work will begin in FY1997 anticipating supplemental funding in FY1997. TRU line work will cease approximately February 28, 1997 should supplemental funding not become available.

- DOE has stated that the FY1999 ADSs may be requested by waste type. This is a significant and currently undefined effort and not included in FY 1997 planning. This may however be included in future outyear planning.

- Contact-handled LLMW stabilization treatment will consist of direct disposal, onsite treatment, and commercial treatment. Transfer of waste to the vendor awarded the CH-LLMW stabilization treatment contract will meet Tri-Party Agreement milestone M-19 on September 30, 1999.

- Trench 34 of burial ground $218-W-5$ will be operated in a standby mode but will not be operated or receive waste for disposal in FY1997. Trench 31 will receive waste in a storage mode during 


\section{SOLID WASTE PROGRAM}

WBS 1.2.1

FY1997, and will transfer to disposal in FY2000.

- A deep trench (approximately $72 \mathrm{ft}$ deep) is planned to be constructed in FY1997 to receive forecasted waste starting in FY1998. This deeper than normal trench provides a higher disposal volume for the same RCRA cover cost.

- Processing required for oversized TRU and LLMW and RH-TRU and RH LLMW will be determined under Tri-Party Agreement Milestone M-91, as defined by the Project Management Plans due in 1999 and 2000. Funding for implementation of the PMP's, therefore, has very little basis.

- The DOE walk-in work account (1A1) is contract-approved work through the MYWP and Work Authorization signatures. Workscope will be detailed throughout the year by DOE-RL.

- Environmental Restoration Program will lead the DNFSB-94-2 composite analysis. SWP effort provides inventory estimates and source term inputs to modeling of parametric data and reviews of data outputs and draft reports. Also, the composite analysis will have no impact on LLW burial ground operations during FY1997 and outyears.

- Part B permit applications for Solid Waste facilities have been submitted to Ecology. Current new facility construction will be accomplished under interim status (Hanford Federal Facility Agreement and Consent Order, 89-10 Rev. 1). It is assumed that final status and approval of all required permits will be obtained to support facility operation. The low level burial grounds closure schedule will be renegotiated prior to approval of the Part B permit.

- The T Plant head end contains miscellaneous LLW, excess chemicals, and abandoned process equipment. This waste poses an industrial and radiological hazard to T Plant personnel during administrative controlled entries for surveillance of the area. Solid Waste Program assumes that the Nuclear Energy program (NE-60) retains responsibility for those chemicals and dispositions them properly. 


\subsection{PROGRAM END POINT TARGETS}

This section identifies the significant end point targets, technical objectives and safety objectives to be achieved in accomplishing the program mission. This includes general and specific objectives, deliverables, or activities that are essential to the program mission. This information provides an overview of the activities and deliverables that are contained in the schedule section.

\subsubsection{End Point Targets}

Table 1.2.1-1 contains the End Point Targets that are established in the Hanford Mission Direction Document. They provide the basis for the program mission and are implemented through technical requirements contained in the Hanford Site Technical Baseline.

Table 1.2.1-1 End Point Targets.

\begin{tabular}{||l|l||}
\hline 1 & $\begin{array}{l}\text { MDD. Central Plateau. Final. 28 } \\
\text { Retrievably stored TRU waste retrieved, processed, shipped offsite to WIPP. }\end{array}$ \\
\hline 2 & $\begin{array}{l}\text { MDD, Central Plateau. Final. 29 } \\
\text { Low-level and low-level mixed waste from onsite and offsite sources (including PNNL special case wastes) will } \\
\text { continue to be disposed of in the } 200 \text { Areas (provided it meets the Hanford Site Solid Waste Acceptance } \\
\text { Criteria). }\end{array}$ \\
\hline 3 & $\begin{array}{l}\text { MDD. Central Plateau. Interim, 30 } \\
\text { Offsite TRU shipments to Hanford for interim storage will be reinstated. }\end{array}$ \\
\hline
\end{tabular}

\subsubsection{Technical Objectives}

Table 1.2.2-1 contains the Technical Objectives that achieve the end point targets. They are established in various Program specific documents and are implemented through technical requirements contained in the Hanford Site Technical Baseline. 
Table 1.2.2-1 Technical Objectives - Solid Waste Programs.

1 Start TRU retrieval project, WRAP TRU line and transfer TRU Waste to WIPP

Retrieve TRU waste beginning 2001 (TPA-M-91) and complete by 2004 (TPA-M-91)

Complete RH LLMW \& TRU Project Management plans by 1999 \& 2000 (TPA-M-91) and implement

Closure of burial grounds per RCRA and Performance Assessment requirements

Remove Spent Fuel from T-Plant

Establish RMW treatment contracts and process waste at TPA M-19 defined throughput rates (Note, Possible acceleration to meet "Alm 10 year plan")

Maintain the radioactive mixed waste burial trenches (Trenches T-31 and T-34 of burial ground $218-W-5$ ). Operation of one trench as storage capacity is planned for 1997-2000.

\subsubsection{Safety Objectives}

Table 1.2.3-1 contains the Safety Objectives that are established in the Mission Direction Document.

Table 1.2.3-1 Safety Objectives.

\begin{tabular}{||l|l||}
\hline 1 & $\begin{array}{l}\text { MDD, Central Core, Safety Objective } \mathbf{S 5 3} \\
\text { Conduct operations and maintenance of the Nonradioactive Waste Storage Facility (Building 616) in cold } \\
\text { standby mode. }\end{array}$ \\
\hline 2 & $\begin{array}{l}\text { MDD. Central Plateau, Safety Objective } \mathbf{S 3 9} \\
\text { Maintain Liquid Waste Tank Car, provide maintenance, repairs, testing and certification. }\end{array}$ \\
\hline 3 & $\begin{array}{l}\text { MDD. Central Plateau. Safety Objective } \mathbf{S 4 0} \\
\text { Maintain and operate WRAP I facility. }\end{array}$ \\
\hline 4 & $\begin{array}{l}\text { MDD. Central Plateau. Safety Objective } \mathbf{S 4 1} \\
\text { Provide surveillance, maintenance and monitoring of contaminated equipment stored in 221-T canyon. }\end{array}$ \\
\hline 5 & $\begin{array}{l}\text { MDD. Central Plateau. Safety Objective } \mathbf{S 4 2} \\
\text { provide decontamination and maintenance services in support of essential safety operations at the 2706-TA } \\
\text { Building: equipment decontamination, LLW, and RMW packaging. }\end{array}$ \\
\hline 6 & $\begin{array}{l}\text { MDD. Central Plateau. Safety Objective } \mathbf{S 4 3} \\
\text { Conduct operations, surveillance, and maintenance of the 224-T TRU Storage and Assay Facility (TRUSAF), the } \\
\text { Central Waste Complex (CWC) storage facilities. }\end{array}$ \\
\hline 7 & $\begin{array}{l}\text { MDD. Central Plateau, Safety Objective } \mathbf{S 4 4} \\
\text { Conduct of operations, surveillance and maintenance of the Low Level Waste Burial Grounds (LLBG) }\end{array}$ \\
\hline
\end{tabular}

\subsection{SUMMARY-LEVEL FORECASTING DATA}

This section contains forecast information about the program inputs and outputs during the program life 
cycle. The forecast information is an integral part of the technical basis for the planning, scheduling, and budgeting process.

\subsubsection{Waste Type Data}

Table 1.3.1-1 contains the waste (Solid Waste, Tank Waste, Liquid Effluents, Special Case Waste) inventory and volume projection data. These data are used to track the waste through generation, transfer, receipt, storage, and disposition. The Program schedule and budget reflect the plans for disposition of waste. 


\section{SOLID WASTE PROGRAM \\ WBS 1.2.1}

EY 1997 Program Plan

WHC-SP-1114.REV 2

Table 1.3.1-1 Waste Volume Inventory

(cubic meters) (Sheet 1 of 16)

\begin{tabular}{|c|c|c|c|c|c|c|c|c|c|c|c|c|c|c|}
\hline & $\begin{array}{l}u w \\
\text { (sol) }\end{array}$ & $\begin{array}{l}\mathrm{UMW} \\
\text { (SOL) }\end{array}$ & TAU & TRUM & HAZ & HLW & $\begin{array}{l}\text { uw } \\
\text { (UO) }\end{array}$ & $\begin{array}{l}\text { LLMW } \\
\text { (LIO) }\end{array}$ & $\begin{array}{l}\text { Industrial } \\
\text { Wasto } \\
\text { Walter }\end{array}$ & $\begin{array}{l}\text { Trealed } \\
\text { Luqud } \\
\text { Ethicent }\end{array}$ & $\begin{array}{l}\text { Sanikary } \\
\text { (LiO) }\end{array}$ & $\begin{array}{l}\text { Sanitary } \\
\text { (5OL) }\end{array}$ & Asbostos & $\begin{array}{l}\text { Spocial } \\
\text { Cas9 } \\
\text { Watt. }\end{array}$ \\
\hline \multicolumn{15}{|l|}{ FY 1997} \\
\hline Begin Imverttory & 122.78 & 0245.52 & 159827 & 2024 & & & & & & & & & & \\
\hline Received & 556325 & 100020 & 410.28 & 101.871 & 4603 & & & & & & & & & \\
\hline Generated & 200.30 & 19.88 & & 7.0 & 1.0 & 113.55 & & & & 2072.0 & & 205 & & \\
\hline \multicolumn{15}{|l|}{ Rechuced } \\
\hline Transferred & & & & & 48.18 & $\mathbf{1 1 3 . 5 6}$ & & & & 20720 & & 25 & & \\
\hline Disposed & 4846.76 & & & & & & & & & & & & & \\
\hline End Inventory & & 808.9 & 16057.7 & 304.15 & & & & & & & & & & \\
\hline \multicolumn{15}{|l|}{ FY 1998} \\
\hline Begin Inventory & & 889.8 & 16357.7 & 391.15 & & & & & & & & & & \\
\hline Received & 4641.31 & 719.457 & 477.326 & 126433 & 41.79 & & & & & & & & & \\
\hline Generated & 224.12 & 19.38 & & & 1.0 & 113.5 & & & & 1514 & & & & \\
\hline Recluced & & & 1174.45 & 16022 & & & & & & & & & & \\
\hline Transferred & & & & & 43.04 & 113.5 & & & & 1514 & & & & \\
\hline Disposed & 306203 & & & & & & & & & & & & & \\
\hline End Inventory & & 9418.03 & 15645.3 & 33824 & & & & & & & & & & \\
\hline \multicolumn{15}{|l|}{ FY 1999} \\
\hline Begin Imventory & & 9418.00 & 15645.3 & 338.24 & & & & & & & & & & \\
\hline Peceived & 438668 & 823349 & 513115 & 134.613 & 41.78 & & & & & & & & & \\
\hline Generated & 325.31 & & 1300.71 & 130.79 & 1.0 & 113.55 & & & & 1514 & & & & \\
\hline Rectuced & & 475.88 & & & & & & & & & & & $\cdot$ & \\
\hline Transterred & & & 118289 & 178.96 & 49.98 & 113.55 & & & & 1514 & & & & \\
\hline Disposed & 3738.14 & & & & & & & & & & & & & \\
\hline End Inventory & & 865481 & 16811 & 414.53 & & & & & & & & & & \\
\hline \multicolumn{15}{|l|}{ FY 2000} \\
\hline Begin Inventory & & $9654 . B 1$ & 16311 & 414.53 & & & & & & & & & & \\
\hline Received & 6136.74 & 119124 & 634328 & 100.371 & 41.78 & & & & & & & & & \\
\hline Generated & 260.50 & 24 & 559.8 & 19.92 & 1.0 & 113.55 & & & & 1514 & & & & \\
\hline \multicolumn{15}{|l|}{ Reduced } \\
\hline Transferred & & & 6023 & 56.06 & 42.78 & 113.55 & & & & 1514 & & & & \\
\hline Disposed & 3374.94 & 858.31 & & & & & & & & & & & & \\
\hline End Inventory & & 920.34 & 16028.6 & 408.65 & & & & & & & & & & \\
\hline \multicolumn{15}{|l|}{ FY2001 } \\
\hline Begin Inventory & & 9420.34 & 16826.6 & 468.65 & & & & & & & & & & \\
\hline Received & 5621.04 & 171833 & 221.621 & 306.776 & 41.52 & & & & & & & & & \\
\hline Generated & 23047 & 400.66 & 1354,91 & & & 113.55 & & & & 1325 & & & & \\
\hline Reduced & & & & 13727 & & & & & & & & & & \\
\hline
\end{tabular}


Table 1.3.1-1 Waste Volume Inventory

(cubic meters) (Sheet 2 of 16)

\begin{tabular}{|c|c|c|c|c|c|c|c|c|c|c|c|c|c|c|}
\hline & $\begin{array}{l}\text { 亗 } \\
\text { (SOL) }\end{array}$ & $\underset{\text { (SOL) }}{\mathbb{L}}$ & TAU & TRUM & HAZ & HLW & 㟧 & (LUN) & $\begin{array}{l}\text { Inoustrits } \\
\text { Wosto } \\
\text { Water }\end{array}$ & $\begin{array}{l}\text { Treated } \\
\text { Uats } \\
\text { Efiluent }\end{array}$ & $\begin{array}{l}\text { Santary } \\
\text { (UL) }\end{array}$ & $\begin{array}{l}\text { Santary } \\
(\mathrm{SOO})\end{array}$ & Astostos & $\begin{array}{l}\text { Spacial } \\
\text { Coses } \\
\text { Wasto }\end{array}$ \\
\hline Transferted & & & 847.76 & 43.81 & 4253 & 113.55 & & & & & & & & \\
\hline Disposed & 281432 & 1913.19 & & & & & & & & & & & & \\
\hline End Imentory & & 8273.75 & 17440.1 & 674.21 & & & & & & & & & & \\
\hline \multicolumn{15}{|l|}{ FY 2002} \\
\hline Begin Inventory & & 527375 & 17440.1 & 67421 & & & & & & & & & & \\
\hline Received & 6071.83 & 100521 & 250.178 & 304255 & 42.807 & & & & & & & & & \\
\hline Generated & 367.17 & 1436.49 & 707.07 & & 1.0 & 113.55 & & & & 1325 & & & & \\
\hline Recuced & & & & $2 \boldsymbol{2 0}$ & & & & & & & & & & \\
\hline Transferred & & & 438.26 & 18383 & 43.81 & 113.55 & & & & 1305 & & & & \\
\hline Disposed & 4009.62 & 2925.93 & & & & & & & & & & & & \\
\hline End Inventory & & $\operatorname{ses} 8.13$ & 173418 & 061.43 & & & & & & & & & & \\
\hline \multicolumn{15}{|l|}{ FY 2003} \\
\hline Begin Imventory & & 2258.13 & 179638 & 861.43 & & & & & & & & & & \\
\hline Received & 5297.87 & 134220 & 113285 & 373204 & 43.678 & & & & & & & & & \\
\hline Generated & 355.08 & 1558.60 & 1027.54 & 9.02 & 1.0 & 113.55 & & & & 1325 & & & & \\
\hline \multicolumn{15}{|l|}{ Reduceod } \\
\hline Transferred & & & 524.39 & 189.85 & 44.68 & 113.55 & & & & 1325 & & & & \\
\hline Disposed & 2713.78 & 2008.49 & & & & & & & & & & & & \\
\hline End Inventory & & 8000.43 & 18544.9 & 1043.60 & & & & & & & & & & \\
\hline \multicolumn{15}{|l|}{ FY 2004} \\
\hline Begin Inventory & & 8000.43 & 18549 & 1043.68 & & & & & & & & & & \\
\hline Received & 4944.17 & 1675.51 & 68.436 & 36.016 & 43.679 & & & & & & & & & \\
\hline Generated & 333.67 & 1780.36 & 823.28 & 167.78 & 1.0 & 113.55 & & & & 1305 & & & & \\
\hline \multicolumn{15}{|l|}{ Reduced } \\
\hline Transferred & & & 350.19 & 183.67 & 44.68 & 113.55 & & & & 1325 & & & & \\
\hline Disposed & 2338.65 & 2689.57 & & & & & & & & & & & & \\
\hline End Inventory & & 9661.45 & 19071.2 & 105368 & & & & & & & & & & \\
\hline \multicolumn{15}{|l|}{ FY 2005} \\
\hline Begin Imventory & & 9651.45 & 190712 & 1063.68 & & & & & & & & & & \\
\hline Received & 2747.14 & 2141.03 & B5.94 & 35.008 & 43.421 & & & & & & & & & \\
\hline Generated & 391.88 & 247.35 & 70352 & 128 & 1.0 & 113.55 & & & & 1325 & & & & \\
\hline \multicolumn{15}{|l|}{ Peduced } \\
\hline Transterred & & & 367.59 & 16.14 & 44.42 & 113.55 & & & & 1305 & & & & \\
\hline Disposed & 2006.70 & 1786.60 & & & & & & & & & & & & \\
\hline End imventory & & 105028 & 19667.8 & 1053.68 & & & & & & & & & & \\
\hline \multicolumn{15}{|l|}{ FY2006 } \\
\hline Begin Inventory & & 105288 & 19567.8 & 106360 & & & & & & & & & & \\
\hline
\end{tabular}


Table 1.3.1-1 Waste Volume Inventory

(cubic meters) (Sheet 3 of 16)

\begin{tabular}{|c|c|c|c|c|c|c|c|c|c|c|c|c|c|c|}
\hline & UW & 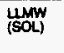 & TRU & TRUM & HAZ & HLW & LW & $\begin{array}{l}\mathrm{LMW} \\
(\mathrm{L} / \mathrm{O})\end{array}$ & $\begin{array}{l}\text { Inctustrial } \\
\text { Whatie } \\
\text { Weter }\end{array}$ & $\begin{array}{l}\text { Treated } \\
\text { Uaght } \\
\text { Efiluent }\end{array}$ & $\begin{array}{l}\text { Santany } \\
\text { (UO) }\end{array}$ & $\begin{array}{l}\text { Santany } \\
\text { (SOL) }\end{array}$ & Astoectose & $\begin{array}{l}\text { Soecha } \\
\text { Case } \\
\text { Wasto }\end{array}$ \\
\hline Received & 272325 & 1705.67 & 62.581 & 30382 & 43,421 & & & & & & & & & \\
\hline Generated & 31821 & 620.04 & 79678 & 4.86 & t.o & $\$ 13.55$ & & & & $\$ 25$ & & & & \\
\hline \multicolumn{15}{|l|}{ Reduced } \\
\hline Transferred & & & 358.02 & 15.12 & 44.42 & 113.56 & & & & xess & & & & \\
\hline Disposed & 2020.81 & 167308 & & & & & & & & & & & & \\
\hline End Inventory & & 100782 & $20058 . \theta$ & 1073.68 & & & & & & & & & & \\
\hline \multicolumn{15}{|l|}{ FY 2007} \\
\hline Begin Inventory & & 100762 & 20058.9 & 107368 & & & & & & & & & & \\
\hline Fecoived & 278.00 & 2002.4 & 69281 & 30.382 & 43.421 & & & & & & & & & \\
\hline Generated & 327.46 & 746.54 & B5028 & 0.16 & 1.0 & 113.55 & & & & tses & & & & \\
\hline \multicolumn{15}{|l|}{ Rectuced } \\
\hline Transferred & & & 30274 & 10.42 & 44.42 & 113.55 & & & & 135 & & & & \\
\hline Disposed & 2206.64 & 1500.74 & & & & & & & & & & & & \\
\hline End Invertitory & & 118038 & 20580.4 & 1083.68 & & & & & & & & & & \\
\hline \multicolumn{15}{|l|}{ FY 2008} \\
\hline Begin inventory & & 118068 & 20580.4 & 1083.68 & & & & & & & & & & \\
\hline Received & 2752.25 & 254484 & 2241 & 30.382 & 43.421 & & & & & & & & & \\
\hline Generated & 335.29 & 656.82 & 045.04 & 0.16 & 1.0 & 113.55 & & & & 1305 & & & & \\
\hline \multicolumn{15}{|l|}{ Reduced } \\
\hline Trensferted & & & 306.49 & 10.42 & 44.42 & 113.55 & & & & 1325 & & & & \\
\hline Disposed & 2279.7 & 151843 & & & & & & & & & & & & \\
\hline End Irwentory & & 13011.5 & 211362 & 1008.68 & & & & & & & & & & \\
\hline \multicolumn{15}{|l|}{ FY 2009} \\
\hline Begin Imventory & & 13011.5 & 211362 & 1083.68 & & & & & & & & & & \\
\hline Received & 250433 & 3174.19 & 22.461 & 10.382 & 32421 & & & & & & & & & \\
\hline Generated & 577.2 & & & & 1.0 & 113.55 & & & & 1325 & & & & \\
\hline Peduced & & 17400 & 377.17 & B1.59 & & & & & & & & & & \\
\hline Transferred & & & 27301 & 10.42 & 34.42 & 113.56 & & & & 1305 & & & & \\
\hline Disposed & 2273.68 & 1235.01 & & & & & & & & & & & & \\
\hline End Invertiony & & 14648.1 & 204932 & 1001.92 & & & & & & & & & & \\
\hline \multicolumn{15}{|l|}{ FY 2010} \\
\hline Begin Inventory & & 14648.1 & 204932 & 1001.82 & & & & & & & & & & \\
\hline Received & 2064.37 & $2 \operatorname{sen} 76$ & 22401 & 10.382 & 33.421 & & & & & & & & & \\
\hline Generated & B15.14 & & & & 1.0 & 113.55 & & & & 1320s & & & & \\
\hline Reduced & & 1126.85 & 1492.91 & 22.1 & & & & & & & & & & \\
\hline Transferred & & & 507.91 & 22.85 & 34.42 & 113.55 & & & & 1320 & & & & \\
\hline Disposed & 251078 & 1356.4 & & & & & & & & & & & & \\
\hline End Inventory & & 14950.9 & 184026 & 88724 & & & & & & & & & & \\
\hline
\end{tabular}


Table 1.3.1-1 Waste Volume Inventory

(cubic meters) (Sheet 4 of 16)

\begin{tabular}{|c|c|c|c|c|c|c|c|c|c|c|c|c|c|c|}
\hline & $\stackrel{u W}{\text { (SOL) }}$ & 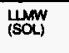 & $\operatorname{TRU}$ & TRUM & HAZ & H.W & $\begin{array}{l}\text { LW } \\
\text { (념) }\end{array}$ & (LiC) & $\begin{array}{l}\text { Incustriel } \\
\text { Waste } \\
\text { Water }\end{array}$ & $\begin{array}{l}\text { Trouted } \\
\text { Livid } \\
\text { Eflivent }\end{array}$ & $\begin{array}{l}\text { Sankary } \\
\text { (uO) }\end{array}$ & $\begin{array}{l}\text { Sentery } \\
\text { (SOL) }\end{array}$ & Asboctos & $\begin{array}{l}\text { Soescil } \\
\text { Comeste }\end{array}$ \\
\hline \multicolumn{15}{|l|}{ Fr 2011} \\
\hline Begin Irventory & & 14850.9 & 18486.6 & 88724 & & & & & & & & & & \\
\hline Received & 434124 & 370830 & 133336 & 18357 & 114.119 & & & & & & & & & \\
\hline Generated & 1387.34 & & & & 1.0 & 11355 & & & & 1325 & & & & \\
\hline Rectuced & & 2127.58 & 132271 & 33.43 & & & & & & & & & & \\
\hline Transferred & & & 870.75 & 13264 & 115.12 & 113.55 & & & & 1300 & & & & \\
\hline Disposed & 5241.86 & 1787.3 & & & & & & & & & & & & \\
\hline End inventory & & 14581.9 & 103023 & 721.43 & & & & & & & & & & \\
\hline \multicolumn{15}{|l|}{ FY 2012} \\
\hline Begin Inventory & & 14581.9 & 168023 & 721.43 & & & & & & & & & & \\
\hline Received & 435724 & 336231 & 133396 & 19.357 & t15.918 & & & & & & & & & \\
\hline Generated & 1300.3 & & & 40.17 & 1.0 & 11355 & & & & 1320 & & & & \\
\hline Recuced & & 1380.19 & 2667.37 & & & & & & & & & & & \\
\hline Transferred & & & 1206.14 & 189.32 & 116.92 & 113.55 & & & & 1305 & & & & \\
\hline Disposed & 5255.84 & 2839.53 & & & & & & & & & & & & \\
\hline End Inventory & 5.1 & 13443 & 12438 & 581.54 & & & & & & & & & & \\
\hline \multicolumn{15}{|l|}{ FY 2013} \\
\hline Begin Inventory & 5.1 & 13443 & 12436 & 581.54 & & & & & & & & & & \\
\hline Received & 5572.81 & 4385.58 & 133.396 & 118.387 & 185.918 & & & & & & & & & \\
\hline Generated & 1506.99 & & & & 1.0 & 113.55 & & & & 1325 & & & & \\
\hline Reduced & & 2041.78 & 1921.17 & 104.81 & & & & & & & & & & \\
\hline Transferted & & & 1181.59 & 140.90 & 16692 & 11355 & & & & 1305 & & & & \\
\hline Disposed & 6KAB & 3348.54 & & & & & & & & & & & & \\
\hline End Inventory & 0.5 & 12190.1 & $\$ 040.4$ & 428.06 & & & & & & & & & & \\
\hline \multicolumn{15}{|l|}{ FY 2014} \\
\hline Begin Inventory & 0.5 & 12199,1 & 8340.4 & 20.06 & & & & & & & & & & \\
\hline Received & 5801.78 & 4682.1 & 134.396 & 120.385 & 166.497 & & & & & & & & & \\
\hline Generated & 1287.8 & & & 100.68 & 1.0 & 113.55 & & & & 1305 & & & & \\
\hline Reduced & & 1782.56 & 109784 & & & & & & & & & & & \\
\hline Transferred & & & 105408 & 267.78 & 967.5 & 113.55 & & & & 1325 & & & & \\
\hline Disposed & 6400.59 & 4021.06 & & & & & & & & & & & & \\
\hline End Inventory & & 1085.5 & 7186.87 & 36223 & & & & & & & & & & \\
\hline \multicolumn{15}{|l|}{ FY 2015} \\
\hline Begin Inventory & & 10257.5 & 7198.87 & 362.23 & & & & & & & & & & \\
\hline Recaived & 5558.55 & 2404.14 & 132396 & 119.357 & 160.497 & & & & & & & & & \\
\hline Generated & 132882 & & & & 1.0 & 11355 & & & & 1325 & & & & \\
\hline Rechuced & & 1113.82 & 2233.54 & 2.08 & & & & & & & & & & \\
\hline Transferred & & & 434.75 & 184.16 & 167.5 & 113.55 & & & & 1200 & & & & \\
\hline
\end{tabular}




\section{SOLID WASTE PROGRAM WBS 1.2.1}

WHC-SP-1114,REV 2

Table 1.3.1-1 Waste Volume Inventory

(cubic meters) (Sheet 5 of 16)

\begin{tabular}{|c|c|c|c|c|c|c|c|c|c|c|c|c|c|c|}
\hline & $\begin{array}{l}\text { LW } \\
\text { (SOL) }\end{array}$ & $\mathrm{WMW}_{\text {(SOL) }}$ & TRU & TRUM & HAZ & Hew. & $\begin{array}{l}\text { uw } \\
\text { (L口) }\end{array}$ & 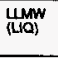 & $\begin{array}{l}\text { Industrial } \\
\text { Waste } \\
\text { Wator }\end{array}$ & $\begin{array}{l}\text { Trueded } \\
\text { Lidid } \\
\text { Ethuent }\end{array}$ & $\begin{array}{l}\text { Santary } \\
\text { (uO) }\end{array}$ & $\begin{array}{l}\text { Sankary } \\
\text { (SOL) }\end{array}$ & Acbestos & $\begin{array}{l}\text { Soectil } \\
\text { Case } \\
\text { Wasto }\end{array}$ \\
\hline Disposed & 6200.88 & 4127.58 & & & & & & & & & & & & \\
\hline End inventory & & 7752.64 & $45 \times 5.78$ & 209.37 & & & & & & & & & & \\
\hline \multicolumn{15}{|l|}{ FY 2016} \\
\hline Begin Inventory & & 775284 & 4535.78 & 289.37 & & & & & & & & & & \\
\hline Received & 5345.83 & 3004.06 & 7.180 & 100.257 & 187.400 & & & & & & & & & \\
\hline Generated & 1353.72 & & & & 1.0 & 113.55 & & & & 1325 & & & & \\
\hline Recluced & & 121529 & 2000.49 & 57.05 & & & & & & & & & & \\
\hline Transferred & & & 515.32 & 507.85 & 168.5 & 113.55 & & & & 1325 & & & & \\
\hline Disposed & 6404.55 & 4050.23 & & & & & & & & & & & & \\
\hline End Imventory & & 5097.87 & 1857.16 & 104.82 & & & & & & & & & & \\
\hline \multicolumn{15}{|l|}{ FY 2017} \\
\hline Begin Inventory & & 5287.87 & 1937.16 & 104.92 & & & & & & & & & & \\
\hline Received & 5184.51 & 205294 & 3.500 & 100257 & 167.490 & & & & & & & & & \\
\hline Generated & 1241.25 & & & 20.61 & 1.0 & 11355 & & & & 1320 & & & & \\
\hline Rechuced & & 815.4 & 1430.74 & & & & & & & & & & & \\
\hline Transferred & & & 502.16 & 294.48 & 168.5 & 113.55 & & & & 1325 & & & & \\
\hline Disposed & 6200.76 & 4115.52 & & & & & & & & & & & & \\
\hline End Inventory & & 2679.60 & 4.86 & 13.31 & & & & & & & & & & \\
\hline \multicolumn{15}{|l|}{ FY 2018} \\
\hline Begin Inventory & & 2878.90 & 4.86 & 13.31 & & & & & & & & & & \\
\hline Received & 5084.51 & 1708.26 & 3.500 & 100.257 & 166.497 & & & & & & & & & \\
\hline Generated & 8003.13 & 2340.56 & 368.9 & 10045 & 1.0 & 113.55 & & & & 1305 & & & & \\
\hline \multicolumn{15}{|l|}{ Reduced } \\
\hline Transferred & & & 390.49 & 20071 & 167.5 & t13.55 & & & & 1325 & & & & \\
\hline Disposed & 502884 & 4048.08 & & & & & & & & & & & & \\
\hline End Imventory & & 248248 & 4.86 & 13.31 & & & & & & & & & & \\
\hline \multicolumn{15}{|l|}{ FY 2019} \\
\hline Begin Inventory & & 248240 & 4.86 & 13.31 & & & & & & & & & & \\
\hline Received & 5068.51 & 1438,68 & 18.180 & 100.257 & 185.661 & & & & & & & & & \\
\hline Generated & 745.58 & 1384.32 & 0.06 & 10.79 & 1.0 & 113.55 & & & & 1330 & & & & \\
\hline \multicolumn{15}{|l|}{ Rectuced } \\
\hline Transferred & & & 3.65 & 111.05 & 166.66 & 113.55 & & & & 1325 & & & & \\
\hline Disposed & 5890.07 & 280273 & & & & & & & & & & & & \\
\hline End Inventory & & 2264.42 & 4.86 & 13.31 & & & & & & & & & & \\
\hline \multicolumn{15}{|l|}{ FY 2020} \\
\hline Recoived & & 2064.42 & 4.66 & 13.31 & & & & & & & & & & \\
\hline Generated & 744.77 & 1021.71 & 0.06 & 10.79 & 1.0 & 113.55 & & & & 1325 & & & & \\
\hline
\end{tabular}


SOLID WASTE PROGRAM

WBS 1.2.1

EY 1997 Program Plan

WHC-SP-1114. REV 2

Table 1.3.1-1 Waste Volume Inventory

(cubic meters) (Sheet 6 of 16)

\begin{tabular}{|c|c|c|c|c|c|c|c|c|c|c|c|c|c|c|}
\hline & $\begin{array}{l}\text { ㄴw } \\
\text { (SOL) }\end{array}$ & WOW & IRU & TRUM & mor & HLW & uw & WMON & $\begin{array}{l}\text { Industrial } \\
\text { Waste } \\
\text { Water }\end{array}$ & $\begin{array}{l}\text { Troeted } \\
\text { Lugid } \\
\text { Effluent }\end{array}$ & $\begin{array}{l}\text { Sanitary } \\
\text { (UQ) }\end{array}$ & $\begin{array}{l}\text { Sentary } \\
\text { (SOL) }\end{array}$ & Albestos & $\begin{array}{l}\text { Souciol } \\
\text { Coese } \\
\text { Waste }\end{array}$ \\
\hline \multicolumn{15}{|l|}{ Pectuced } \\
\hline Transferred & & & 3.5 & 111.06 & 16066 & 11355 & & & & 1305 & & & & \\
\hline Disposed & 565828 & 2407.23 & & & & & & & & & & & & \\
\hline End Imventory & & 210025 & 4.86 & 13.31 & & & & & & & & & & \\
\hline \multicolumn{15}{|l|}{ FY 2021} \\
\hline Begin Irventory & & 2100.25 & 4.86 & $13.3 \%$ & & & & & & & & & & \\
\hline Received & $5022.7 t$ & 178325 & 22.600 & 100.457 & 163.679 & & & & & & & & & \\
\hline Generated & 743.34 & 860.58 & 0.06 & 10.79 & 1.0 & 113.55 & & & & 1325 & & & & \\
\hline \multicolumn{15}{|l|}{ Reduced } \\
\hline Transferred & & & 3.60 & 111.05 & 164.68 & 113.55 & & & & 1325 & & & & \\
\hline Disposed & 550285 & 2287.15 & & & & & & & & & & & & \\
\hline End Inventory & & 1981.60 & 4.66 & 13.31 & & & & & & & & & & \\
\hline \multicolumn{15}{|l|}{ FY 2022} \\
\hline Begin Inventory & & 1981.63 & 4.66 & $\mathbf{1 3 . 3 1}$ & & & & & & & & & & \\
\hline Received & 2280.71 & 102035 & 29.600 & 100.457 & 80.706 & & & & & & & & & \\
\hline Generated & 36726 & 1334.56 & 0.06 & 10.79 & 1.0 & 113.55 & & & & 1325 & & & & \\
\hline \multicolumn{15}{|l|}{ Reduced } \\
\hline Transferred & & & 3.65 & 111.05 & 64.71 & 11355 & & & & 1205 & & & & \\
\hline Disposed & 2414.77 & 2133 & & & & & & & & & & & & \\
\hline End Inventory & & 1863.64 & 4.06 & 13.31 & & & & & & & & & & \\
\hline \multicolumn{15}{|l|}{ FY 2023} \\
\hline Begin inventory & & 186364 & 4.60 & 13.31 & & & & & & & & & & \\
\hline Received & 2280.21 & 836.906 & 47.69 & 104.357 & 62.704 & & & & & & & & & \\
\hline Generated & 38483 & 496.73 & 0.06 & 10.79 & 1.0 & $\uparrow 13.55$ & & & & 12es & & & & \\
\hline \multicolumn{15}{|l|}{ Rectuced } \\
\hline Transterred & & & 3.65 & 111.05 & 63.7 & 113.55 & & & & 1305 & & & & \\
\hline Disposed & 2300.34 & 1270.00 & & & & & & & & & & & & \\
\hline End Inventory & & 1748.19 & 4.86 & 13.31 & & & & & & & & & & \\
\hline \multicolumn{15}{|l|}{ FY 2024} \\
\hline Begin Imventory & & 1748.19 & 4.86 & 13.31 & & & & & & & & & & \\
\hline Received & 224121 & 644305 & 34,000 & 105.365 & 61.732 & & & & & & & & & \\
\hline Generated & 384.03 & 479.07 & & 9.78 & 1.0 & 113.55 & & & & 1300 & & & & \\
\hline Rectured & & & 0.94 & & & & & & & & & & & \\
\hline Transferred & & & 3.65 & 111.05 & 62.73 & 113.55 & & & & 1325 & & & & \\
\hline Disposed & 234754 & 1225.52 & & & & & & & & & & & & \\
\hline End Inventory & & $1020 \pi$ & 4.86 & 13.31 & & & & & & & & & & \\
\hline \multicolumn{15}{|l|}{ FY 2024} \\
\hline Begin Inventory & & 1627.07 & 4.86 & $13.3 t$ & & & & & & & & & & \\
\hline
\end{tabular}


SOLID WASTE PROGRAM

WBS 1.2.1

EY 1997 Program Plan

WHC-SP-1114.REV . 2

Table 1.3.1-1 Waste Volume Inventory

(cubic meters) (Sheet 7 of 16)

\begin{tabular}{|c|c|c|c|c|c|c|c|c|c|c|c|c|c|c|}
\hline & $\begin{array}{l}\text { UW } \\
\text { (SOL) }\end{array}$ & $\begin{array}{l}\text { LuW } \\
\text { (SOL) }\end{array}$ & TRU & TAUM & $H N 2$ & HLW & 㟧保) & (U⿺辶) & $\begin{array}{l}\text { Industort } \\
\text { Whate } \\
\text { Water }\end{array}$ & $\begin{array}{l}\text { Treated } \\
\text { Ligid } \\
\text { Efiluent }\end{array}$ & $\begin{array}{l}\text { Sentary } \\
\text { (UIQ) }\end{array}$ & $\begin{array}{l}\text { Sankeary } \\
(\mathrm{SO} \mathrm{L})\end{array}$ & Astoetos & $\begin{array}{l}\text { Special } \\
\text { Cese } \\
\text { Waste }\end{array}$ \\
\hline Received & 224124 & 645356 & 34.008 & 105.386 & 61.372 & & & & & & & & & \\
\hline Generated & 301.03 & 470.07 & & 9.78 & 1.0 & 113.55 & & & & 13205 & & & & \\
\hline Reduced & & & 0.94 & & & & & & & & & & & \\
\hline Transierred & & & 3.65 & 111.05 & 6273 & 11355 & & & & 1325 & & & & \\
\hline Disposed & 2347.54 & 1235.52 & & & & & & & & & & & & \\
\hline End Imentiony & & 1628.07 & 4.88 & 13.31 & & & & & & & & & & \\
\hline \multicolumn{15}{|l|}{ FY 2025} \\
\hline Begin Imventory & & 1626.07 & 4.86 & 13.31 & & & & & & & & & & \\
\hline Received & 2430.54 & 50.50 & 67.790 & 111.957 & 60.552 & & & & & & & & & \\
\hline Generated & $361.6 t$ & 458.08 & 1.07 & 11.81 & 1.0 & 11355 & & & & 1325 & & & & \\
\hline \multicolumn{15}{|l|}{ Reduced } \\
\hline Transferred & & & 4.67 & 11207 & 61.55 & 113.55 & & & & 1305 & & & & \\
\hline Disposed & 2469.57 & 1187.32 & & & & & & & & & & & & \\
\hline End Inventory & & 150699 & 4.86 & 13.31 & & & & & & & & & & \\
\hline \multicolumn{15}{|l|}{ FY 2026} \\
\hline Begin Inventory & & 150600 & 4.86 & 13.31 & & & & & & & & & & \\
\hline Peceived & 2128.46 & 615333 & 56.298 & 111.757 & 55.723 & & & & & & & & & \\
\hline Generated & 360.88 & 421.75 & 0.06 & 10.79 & 1.0 & 113.55 & & & & 1325 & & & & \\
\hline \multicolumn{15}{|l|}{ Reduced } \\
\hline Transferred & & & 3.66 & 111.06 & 56.72 & 113.55 & & & & 13205 & & & & \\
\hline Disposed & $2372 B 4$ & $1145 \pi$ & & & & & & & & & & & & \\
\hline End Inventory & & 138380 & 4.86 & 13.31 & & & & & & & & & & \\
\hline \multicolumn{15}{|l|}{ FY 2027} \\
\hline Begin Inventory & & 138380 & 4.86 & 13.31 & & & & & & & & & & \\
\hline Received & 2129.36 & 609.791 & 62289 & 112657 & 58.723 & & & & & & & & & \\
\hline Generated & 300.80 & 415.54 & 0.06 & 10.78 & 1.0 & 113.55 & & & & 1325 & & & & \\
\hline \multicolumn{15}{|l|}{ Recuced } \\
\hline Transterted & & & 3.65 & 111.05 & 56.72 & 11355 & & & & 1320 & & & & \\
\hline Disposed & 2364.85 & 1137.06 & & & & & & & & & & & & \\
\hline End inventory & & 1258.8 & 4.86 & 13.31 & & & & & & & & & & \\
\hline \multicolumn{15}{|l|}{ FY 2028} \\
\hline Begin Inventory & & 12588 & 4.86 & 13.31 & & & & & & & & & & \\
\hline Received & 1770.16 & 591.836 & 44208 & 108.757 & 55.723 & & & & & & & & & \\
\hline Generated & 261.97 & 418.79 & 0.06 & 10.79 & & 11355 & & & & 1325 & & & & \\
\hline \multicolumn{15}{|l|}{ Reduced } \\
\hline Transferred & & & 3.65 & 111.05 & 56.72 & 113.55 & & & & 1325 & & & & \\
\hline Disposed & 194123 & 180.63 & & & & & & & & & & & & \\
\hline End Inventory & & 1135.5 & 4.86 & 13.31 & & & & & & & & & & \\
\hline
\end{tabular}


Table 1.3.1-1 Waste Volume Inventory

(cubic meters) (Sheet 8 of 16)

\begin{tabular}{|c|c|c|c|c|c|c|c|c|c|c|c|c|c|c|}
\hline & $\begin{array}{l}\mathrm{uW} \\
\text { (SOL) }\end{array}$ & (SOL) & IRU & TRUM & HAZ & H.W & $\begin{array}{l}\text { uW } \\
\text { (ㄴo) }\end{array}$ & $\begin{array}{l}\text { Lumw } \\
\text { (LIO) }\end{array}$ & $\begin{array}{l}\text { Indusstal } \\
\text { Wasto } \\
\text { Weiter }\end{array}$ & $\begin{array}{l}\text { Treated } \\
\text { Lquid } \\
\text { Eftiuent }\end{array}$ & $\begin{array}{l}\text { Santary } \\
\text { (UQ) }\end{array}$ & $\begin{array}{l}\text { Senitery } \\
\text { (SOL) }\end{array}$ & Astostios & $\begin{array}{l}\text { Special } \\
\text { Case } \\
\text { Waste }\end{array}$ \\
\hline \multicolumn{15}{|l|}{ FY 2029} \\
\hline Begin Imentory & & 1135.5 & 4.86 & 13.31 & & & & & & & & & & \\
\hline Received & 579.562 & 104.978 & 50.800 & 10257 & 4.57 & & & & & & & & & \\
\hline Generated & 11.97 & 863.62 & 0.06 & $110 . \pi$ & & 113.55 & & & & 1325 & & & & \\
\hline \multicolumn{15}{|l|}{ Reduced } \\
\hline Transferred & & & 3.65 & 111.05 & 4.57 & 11355 & & & & 1325 & & & & \\
\hline Disposed & 48424 & 110388 & & & & & & & & & & & & \\
\hline End Imventory & & 906.78 & 4.86 & 13.31 & & & & & & & & & & \\
\hline \multicolumn{15}{|l|}{ FY 2030} \\
\hline Begin Inventory & & 096.70 & 4.60 & 13.31 & & & & & & & & & & \\
\hline Received & 481.121 & 68224 & 2315 & 2.657 & 1.229 & & & & & & & & & \\
\hline Generated & 12.96 & 11724 & & & & & & & & & & . & & \\
\hline Rectuced & & & 6.6 & & & & & & & & & & & \\
\hline Transfened & & & 8.65 & 0.28 & 1.23 & & & & & & & & & \\
\hline Disposed & 41.9 & 321.19 & & & & & & & & & & & & \\
\hline End Inventory & & ese.cs & 4.66 & 13.31 & & & & & & & & & & \\
\hline \multicolumn{15}{|l|}{ FY 2031} \\
\hline Begin Inventory & & 858.05 & 4.60 & 13.31 & & & & & & & & & & \\
\hline Received & 454.868 & 63.724 & 25.06 & 2.967 & 1229 & & & & . & & & & & \\
\hline Generated & 12.84 & 61.14 & & & & & & & & & & & & \\
\hline Rectuced & & & 3.87 & & & & & & & & & & & \\
\hline Transferted & & & 6.56 & 0.28 & 123 & & & & & & & & & \\
\hline Disposed & 43338 & 2567 & & & & & & & & & & & & \\
\hline End Inventory & & 723.72 & 4.86 & 13.31 & & & & & & & & & & \\
\hline \multicolumn{15}{|l|}{ FY 2032} \\
\hline Begin Inventory & & 20172 & 4.86 & 13.31 & & & & & & & & & & \\
\hline Received & 437.038 & 59.724 & 1905 & 2057 & 0257 & & & & & & & & & \\
\hline Generated & 1285 & 34.94 & & & & & & & & & & & & \\
\hline Rechued & & & 3.87 & & & & & & & & & & & \\
\hline Transferred & & & 6.58 & 0.28 & 0.26 & & & & & & & & & \\
\hline Disposed & 426.89 & 241.01 & & & & & & & & & & & & \\
\hline End Inventory & & 575.87 & 4.58 & 13.31 & & & & & & & & & & \\
\hline \multicolumn{15}{|l|}{ FY 2033} \\
\hline Begin inventory & & 575.87 & 4.86 & 13.31 & & & & & & & & & & \\
\hline Received & 443.138 & 65.284 & 28.65 & 3.467 & 0.257 & & & & & & & & & \\
\hline Generated & 1296 & 70.32 & & & & & & & & & & & & \\
\hline Recuced & & & 387 & & & & & & & & & & & \\
\hline Transferred & & & 6.58 & 0.26 & 0.20 & & & & & & & & & \\
\hline
\end{tabular}


Table 1.3.1-1 Waste Volume Inventory

(cubic meters) (Sheet 9 of 16)

\begin{tabular}{|c|c|c|c|c|c|c|c|c|c|c|c|c|c|c|}
\hline & $\begin{array}{l}\text { UW } \\
\text { (SOL) }\end{array}$ & $\begin{array}{l}\text { UMW } \\
\text { (SOL) }\end{array}$ & TRU & TRUM & HAZ & Huw & 宸W & (unW & $\begin{array}{l}\text { Induatral } \\
\text { Waste } \\
\text { Water }\end{array}$ & $\begin{array}{l}\text { Treated } \\
\text { Uefid } \\
\text { Effluent }\end{array}$ & $\begin{array}{l}\text { Santary } \\
\text { (UQ) }\end{array}$ & $\begin{array}{l}\text { Sankary } \\
(\mathrm{SOL})\end{array}$ & Ascostos & $\begin{array}{l}\text { Special } \\
\text { Case } \\
\text { Waste }\end{array}$ \\
\hline Disposed & 418.8 & 25214 & & & & & & & & & & & & \\
\hline End Inventory & & 443.14 & 4.66 & 13.31 & & & & & & & & & & \\
\hline \multicolumn{15}{|l|}{ FY 2034} \\
\hline Begin Inventory & & 443.14 & 4.86 & 13.31 & & & & & & & & & & \\
\hline Received & 430.509 & 65294 & 2286 & 2.065 & 0.257 & & & & & & & & & \\
\hline Generated & 1292 & 35.71 & & & & & & & & & & & & \\
\hline Reduced & & & 4.87 & 1.0 & & & & & & & & & & \\
\hline Transferted & & & 6.58 & 0.28 & 0.26 & & & & & & & & & \\
\hline Disposed & 431.71 & 200.08 & & & & & & & & & & & & \\
\hline End Inventory & & 297.87 & 4.86 & 13.31 & & & & & & & & & & \\
\hline \multicolumn{15}{|l|}{ FY 2035} \\
\hline Begin Inventory & & 297.87 & 4.86 & 13.31 & & & & & & & & & & \\
\hline Received & 436.781 & 61.429 & 11.5 & 1.857 & & & & & & & & & & \\
\hline Generated & 10.32 & 48.06 & 7.6 & 1.29 & & & & & & & & & & \\
\hline \multicolumn{15}{|l|}{ Reduced } \\
\hline Transferred & & & 7.6 & 1.28 & & & & & & & & & & \\
\hline Disposed & 42828 & 296.9 & & & & & & & & & & & & \\
\hline End Inventory & & 164.48 & 4.86 & $\mathbf{t 3 . 5 7}$ & & & & & & & & & & \\
\hline \multicolumn{15}{|l|}{ FY 2036} \\
\hline Begin Inventory & & 164.48 & 4.86 & 13.57 & & & & & & & & & & \\
\hline Received & 430.061 & 60.829 & 9.6 & 1.657 & & & & & & & & & & \\
\hline Generated & 10.32 & 175.80 & & & & & & & & & & & & \\
\hline \multicolumn{15}{|l|}{ Reduced } \\
\hline \multicolumn{15}{|l|}{ Transterred } \\
\hline Disposed & 428.28 & 221.06 & & & & & & & & & & & & \\
\hline End Inventory & & 164.48 & 4.86 & 13.63 & & & & & & & & & & \\
\hline \multicolumn{15}{|l|}{ FY 2037} \\
\hline Begin Inventory & & 164.48 & 4.66 & 13.83 & & & & & & & & & & \\
\hline Received & 417.211 & 60.829 & 8.6 & 1.65 & & & & & & & & & & \\
\hline Generated & 10.36 & 35.43 & & & & & & & & & & & & \\
\hline \multicolumn{15}{|l|}{ Reduced } \\
\hline \multicolumn{15}{|l|}{ Transferred } \\
\hline Disposed & 413.47 & 80.68 & & & & & & & & & & & & \\
\hline End Invantory & & 164.48 & 4.86 & 14.00 & & & & & & & & & & \\
\hline \multicolumn{15}{|l|}{ FY 2038} \\
\hline Begin Inventory & & 164.48 & 4.86 & 14.00 & & & & & & & & & & \\
\hline Received & 403.11 & 45.229 & & 0.257 & & & & & & & & & & \\
\hline
\end{tabular}


Table 1.3.1-1 Waste Volume Inventory

(cubic meters) (Sheet 10 of 16)

\begin{tabular}{|c|c|c|c|c|c|c|c|c|c|c|c|c|c|c|}
\hline & $\frac{4 W}{(5 O)}$ & $\begin{array}{l}\mathrm{LMW} \\
\text { (SOL) }\end{array}$ & TRU & TRUM & HNZ & Haw & 㟧W & (um & \begin{tabular}{|l} 
Incluestral \\
Wasto \\
Water
\end{tabular} & 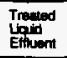 & $\begin{array}{l}\text { Santiany } \\
\text { (UO) }\end{array}$ & $\begin{array}{l}\text { Sanking } \\
\text { (sol) }\end{array}$ & Asbeatios & $\begin{array}{l}\text { Sounide } \\
\text { Comate } \\
\text { Waste }\end{array}$ \\
\hline Generated & 10.36 & 35.40 & & & & & & & & & & & & \\
\hline \multicolumn{15}{|l|}{ Recuced } \\
\hline \multicolumn{15}{|l|}{ Transterred } \\
\hline Disposed & 413,47 & 80.68 & & & & & & & & & & & & \\
\hline End inventory & & 184.48 & 4.86 & 1436 & & & & & & & & & & \\
\hline \multicolumn{15}{|l|}{ FY 2039} \\
\hline Begin Inventory & & 184.48 & 4.86 & 14.36 & & & & & & & & & & \\
\hline Recelved & 400.11 & 45220 & & 0257 & & & & & & & & & & \\
\hline Generated & 10.38 & 36.43 & . & & & & & & & & & & & \\
\hline \multicolumn{15}{|l|}{ Reduced } \\
\hline \multicolumn{15}{|l|}{ Transferred } \\
\hline Disposed & 413.47 & 80.86 & & & & & & & & & & & & \\
\hline End Inventory & & 164.40 & 4.86 & 14.62 & & & & & & & & & & \\
\hline \multicolumn{15}{|l|}{ FY 2040} \\
\hline Begin inventory & & 164.48 & 4.86 & 14.62 & & & & & & & & & & \\
\hline Recoived & 408.11 & 45229 & & 0.257 & & & & & & & & & & \\
\hline Generated & 10.36 & 35.43 & & & & & & & & & & & & \\
\hline \multicolumn{15}{|l|}{ Recuced } \\
\hline \multicolumn{15}{|l|}{ Trensferred } \\
\hline Disposed & 413.47 & 80.66 & & & & & & & & & & & & \\
\hline End Inventory & & 164.48 & 4.86 & 14.88 & & & & & & & & & & \\
\hline \multicolumn{15}{|l|}{ FY 2041} \\
\hline Begin Inventory & & 164.48 & 4.86 & 14.86 & & & & & & & & & & \\
\hline Received & 403.11 & 45229 & & 0257 & & & & & & & & & & \\
\hline Genereted & 10.36 & 36.43 & & & & & & & & & & & & \\
\hline \multicolumn{15}{|l|}{ Reduced } \\
\hline \multicolumn{15}{|l|}{ Transferred } \\
\hline Disposed & 413.47 & 80.86 & & & & & & & & & & & & \\
\hline End Imventory & & 164.48 & 4.86 & 15.14 & & & & & & & & & & \\
\hline \multicolumn{15}{|l|}{ FY 2042} \\
\hline Begin Inventory & & 184.48 & 4.88 & 15.14 & & & & & & & & & & \\
\hline Received & 400.11 & 45220 & & 0.257 & & & & & & & & & & \\
\hline Generated & 10.36 & 35.43 & & & & & & & & & & & & \\
\hline \multicolumn{15}{|l|}{ Reduced } \\
\hline \multicolumn{15}{|l|}{ Transferred } \\
\hline Disposed & 413,47 & 80.68 & & & & & & & & & & & & \\
\hline End Imventory & & 164.48 & 4.86 & 15.4 & & & & & & & & & & \\
\hline
\end{tabular}


Table 1.3.1-1 Waste Volume Inventory

(cubic meters) (Sheet 11 of 16)

\begin{tabular}{|c|c|c|c|c|c|c|c|c|c|c|c|c|c|c|}
\hline & $\begin{array}{l}\text { uw } \\
\text { (sot) }\end{array}$ & (SOL) & RRU & TRUM & muZ & H.W & Uw & WuW & $\begin{array}{l}\text { Industrins } \\
\text { Whate } \\
\text { Water }\end{array}$ & $\begin{array}{l}\text { Trealed } \\
\text { Lugid } \\
\text { Etiluent }\end{array}$ & $\begin{array}{l}\text { Santiary } \\
\text { (UO) }\end{array}$ & $\begin{array}{l}\text { Sanhlary } \\
\text { (SOL) }\end{array}$ & Asbetcos & $\begin{array}{l}\text { Speciel } \\
\text { Cene } \\
\text { Wate }\end{array}$ \\
\hline \multicolumn{15}{|l|}{$F_{22043}$} \\
\hline Begin Inventory & & 164.48 & 4.86 & 15.4 & & & & & & & & & & \\
\hline Received & 400.11 & 45.220 & & 0.257 & & & & & & & & & & \\
\hline Generated & 10.38 & 35.43 & & & & & & & & & & & & \\
\hline \multicolumn{15}{|l|}{ Recuced } \\
\hline \multicolumn{15}{|l|}{ Transferred } \\
\hline Disposed & 413.47 & 80.68 & & & & & & & & & & & & \\
\hline End Invantory & & 164.48 & 4.86 & 15.66 & & & & & & & & & & \\
\hline \multicolumn{15}{|l|}{ FY 2044} \\
\hline Begin Inventory & & 164.48 & 4.86 & 15.66 & & & & & & & & & & \\
\hline Received & 403.11 & 45.220 & 1.0 & 1296 & & & & & & & & & & \\
\hline Generated & 10.36 & 36.43 & 0.08 & 0.00 & & & & & & & & & & \\
\hline \multicolumn{15}{|l|}{ Rectuced } \\
\hline \multicolumn{15}{|l|}{ Transferred } \\
\hline Disposed & 413.47 & 80.66 & & & & & & & & & & & & \\
\hline End Inventory & & 16.48 & 5.68 & 16.94 & & & & & & & & & & \\
\hline \multicolumn{15}{|l|}{ FY 2045} \\
\hline Begin Inventory & & 164.48 & 4.86 & 16.84 & & & & & & & & & & \\
\hline Received & 403.11 & 45220 & & 0.257 & & & & & & & & & & \\
\hline Generated & 10.36 & 35.43 & & & & & & & & & & & & \\
\hline \multicolumn{15}{|l|}{ Rectuced } \\
\hline \multicolumn{15}{|l|}{ Transferred } \\
\hline Disposed & 413.47 & 80.68 & & & & & & & & . & & & & \\
\hline End Inventory & & 164.48 & 5.88 & 17.81 & & & & & & & & & & \\
\hline \multicolumn{15}{|l|}{ FY 2046} \\
\hline Begin Inventory & & 164.46 & 5.80 & 17.91 & & & & & & & & & & \\
\hline Received & 403.111 & 45220 & & 0.257 & & & & & & & & & & \\
\hline Generated & 10.36 & 36.43 & & & & & & & & & & & & \\
\hline \multicolumn{15}{|l|}{ Rechuced } \\
\hline \multicolumn{15}{|l|}{ Transferred } \\
\hline Disposed & 413,47 & 60.68 & & & & & & & & & & & & \\
\hline End Inventory & & 164.48 & 5.68 & 17.47 & & & & & & & & & & \\
\hline \multicolumn{15}{|l|}{ Fr 2047} \\
\hline Begin inventory & & 164.48 & 5.08 & 17.47 & & & & & & & & & & \\
\hline Received & $403+11$ & 45.229 & & 0.257 & & & & & & & & & & \\
\hline Generated & 10.36 & 35.43 & & & & & & & & & & & & \\
\hline \multicolumn{15}{|l|}{ Rechuced } \\
\hline Transferred & & & & & & & & & & & & & & \\
\hline
\end{tabular}


Table 1.3.1-1 Waste Volume Inventory

(cubic meters) (Sheet 12 of 16)

\begin{tabular}{|c|c|c|c|c|c|c|c|c|c|c|c|c|c|c|}
\hline & (SOL) & $\frac{\mathrm{LWMW}}{\text { (SOL) }}$ & TमU & TRUM & HAZ & HLW & $\begin{array}{l}\text { UW } \\
\text { (LiO) }\end{array}$ & $\begin{array}{l}\text { WMW } \\
\text { (UO) }\end{array}$ & $\begin{array}{l}\text { Industrital } \\
\text { Waate } \\
\text { Water }\end{array}$ & $\begin{array}{l}\text { Treated } \\
\text { Luquid } \\
\text { Ethlivent }\end{array}$ & $\begin{array}{l}\text { Sanflary } \\
\text { (UO) }\end{array}$ & $\begin{array}{l}\text { Santary } \\
\text { (SoL) }\end{array}$ & Albestos & $\begin{array}{l}\text { Special } \\
\text { Case } \\
\text { Waste }\end{array}$ \\
\hline Disposed & 413.47 & 60.66 & & & & & & & & & & & & \\
\hline End Inventory & & 764.48 & 5.88 & 17.99 & & & & & & & & & & \\
\hline \multicolumn{15}{|l|}{ FY 2048 } \\
\hline Begin Imentory & & 164.48 & 5.88 & 17.90 & & & & & & & & & & \\
\hline Received & 403.111 & 45.229 & & 0.201 & & & & & & & & & & \\
\hline Generated & 10.36 & 35.43 & & & & & & & & & & & & \\
\hline \multicolumn{15}{|l|}{ Rectuced } \\
\hline \multicolumn{15}{|l|}{ Transterred } \\
\hline Disposed & 413,47 & 80.66 & & & & & & & & & & & & \\
\hline End Imventory & & 164.48 & 5.88 & 17.00 & & & & & & & & & & \\
\hline \multicolumn{15}{|l|}{ FY 2049} \\
\hline Begin Inventory & & 164.48 & 5.88 & 17.90 & & & & & & & & & & \\
\hline Received & 403.111 & 45.229 & & 0.257 & & & & & & & & & & \\
\hline Generated & 10.36 & 35.43 & & & & & & & & & & & & \\
\hline \multicolumn{15}{|l|}{ Reduced } \\
\hline \multicolumn{15}{|l|}{ Transferred } \\
\hline Disposed & 413.47 & 80.68 & & & & & & & & & & & & \\
\hline End Imventory & & 184.48 & 5.80 & 1825 & & & & & & & & & & \\
\hline \multicolumn{15}{|l|}{ FY 2050} \\
\hline Begin Inventory & & 164.48 & 5.88 & 18.25 & & & & & & & & & & \\
\hline Received & 403111 & 45.229 & & 0.257 & & & & & & & & & & \\
\hline Generated & 10.36 & 35.43 & & & & & & & & & & & & \\
\hline \multicolumn{15}{|l|}{ Reduced } \\
\hline \multicolumn{15}{|l|}{ Transferred } \\
\hline Disposed & 413.47 & 80.68 & & & & & & & & & & & & \\
\hline End Inventory & & 164.40 & 5.60 & 18.51 & & & & & & & & & & \\
\hline \multicolumn{15}{|l|}{ FY 2051 } \\
\hline Begin Inventory & & 164.48 & 5.88 & 18.51 & & & & & & & & & & \\
\hline Peceived & 402.111 & 45.229 & & 0.257 & & & & & & & & & & \\
\hline Generated & 10.36 & 35.43 & & & & & & & & & & & & \\
\hline \multicolumn{15}{|l|}{ Recuced } \\
\hline \multicolumn{15}{|l|}{ Transterred } \\
\hline Disposed & 413.47 & 80.88 & & & & & & & & & & & & \\
\hline End Inventory & & 164.40 & 5.80 & 18.77 & & & & & & & & & & \\
\hline
\end{tabular}


Table 1.3.1-1 Waste Volume Inventory

(cubic meters) (Sheet 13 of 16)

\begin{tabular}{|c|c|c|c|c|c|c|c|c|c|c|c|c|c|c|}
\hline & $\begin{array}{l}\text { UW } \\
\text { (SOL) }\end{array}$ & $\begin{array}{l}\text { WunN } \\
\text { (SOL) }\end{array}$ & TAU & TRUM & MNZ & HLW & 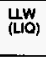 & 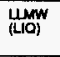 & $\begin{array}{l}\text { Industrial } \\
\text { Wease } \\
\text { Waler }\end{array}$ & $\begin{array}{l}\text { Treated } \\
\text { Lavd } \\
\text { Eftuent }\end{array}$ & $\begin{array}{l}\text { Senitary } \\
\text { (UO) }\end{array}$ & $\begin{array}{l}\text { Seritary } \\
\text { (SOL) }\end{array}$ & Neboutos & $\begin{array}{l}\text { Special } \\
\text { Caese } \\
\text { Waste }\end{array}$ \\
\hline \multicolumn{15}{|l|}{ FY 2052} \\
\hline Begin Inventory & & 164,48 & 5.88 & $18 \pi 7$ & & & & & & & & & & \\
\hline Recelved & 403.111 & 45220 & & 0.257 & & & & & & & & & & \\
\hline Generated & to.36 & 35.43 & & & & & & & & & & & & \\
\hline \multicolumn{15}{|l|}{ Reduced } \\
\hline \multicolumn{15}{|l|}{ Transferred } \\
\hline Disposed & 413.47 & 60.66 & & & & & & & & & & & & \\
\hline End Imventory & & 164.48 & 5.88 & 19.00 & & & & & & & & & & \\
\hline \multicolumn{15}{|l|}{ FY 2053} \\
\hline Begin Inventory & & 164.48 & 5.86 & 19.00 & & & & & & & & & & \\
\hline Received & 400.111 & 45.220 & & 0.257 & & & & & & & & & & \\
\hline Generated & 10.36 & 35.43 & & & & & & & & & & & & \\
\hline \multicolumn{15}{|l|}{ Reduced } \\
\hline \multicolumn{15}{|l|}{ Transferred } \\
\hline Disposed & 413.47 & $\infty 0.68$ & & & & & & & & & & & & \\
\hline End Inventory & & 164.48 & 5.88 & 1929 & & & & & & & & & & \\
\hline \multicolumn{15}{|l|}{ FY 2054} \\
\hline Begin Inventory & & 164.48 & 5.88 & 1929 & & & & & & & & & & \\
\hline Received & 400.111 & 45200 & 1.0 & 0.287 & & & & & & & & & & \\
\hline Generated & 10.38 & 35.43 & 0.01 & 0.02 & & & & & & & & & & \\
\hline \multicolumn{15}{|l|}{ Recuced } \\
\hline \multicolumn{15}{|l|}{ Transferred } \\
\hline Disposed & 413.47 & 80.66 & & & & & & & & & & & & \\
\hline End Inventory & & 164.48 & 689 & 20.58 & & & & & & & & & & \\
\hline \multicolumn{15}{|l|}{ FY 2055} \\
\hline Begin Imventory & & 184.48 & 6.89 & 20.58 & & & & & & & & & & \\
\hline Recelved & 400.111 & 45.220 & & 0.257 & & & & & & & & & & \\
\hline Generated & 10.56 & 35.43 & & & & & & & & & & & & \\
\hline \multicolumn{15}{|l|}{ Recuced } \\
\hline \multicolumn{15}{|l|}{ Transferred } \\
\hline Disposed & 413.47 & 80.68 & & & & & & & & & & & & \\
\hline End Inventory & & 164.48 & 6.89 & 20.84 & & & & & & & & & & \\
\hline \multicolumn{15}{|l|}{ FY 2056} \\
\hline Begin Inventory & & 164.48 & 6.89 & 20.84 & & & & & & & & & & \\
\hline Received & 403111 & 45.220 & & 0257 & & & & & & & & & & \\
\hline Generated & 10.36 & 35.43 & & & & & & & & & & & & \\
\hline \multicolumn{15}{|l|}{ Recurced } \\
\hline Transferred & & & & & & & & & & & & & & \\
\hline
\end{tabular}


Table 1.3.1-1 Waste Volume Inventory

(cubic meters) (Sheet 14 of 16)

\begin{tabular}{|c|c|c|c|c|c|c|c|c|c|c|c|c|c|c|}
\hline & $\frac{L W}{(50)}$ & $\begin{array}{l}\text { WMW } \\
\text { (SOL) }\end{array}$ & TRU & IRUM & HAZ & HLW & Uw & 岕OW & $\begin{array}{l}\text { Incusarina } \\
\text { Weste } \\
\text { Water }\end{array}$ & $\begin{array}{l}\text { Treated } \\
\text { Lutd } \\
\text { Ethivent }\end{array}$ & $\begin{array}{l}\text { Santary } \\
\text { (UQ) }\end{array}$ & $\begin{array}{l}\text { Sanitary } \\
\text { (SOL) }\end{array}$ & Asbeetos & $\begin{array}{l}\text { Spacial } \\
\text { Casa } \\
\text { Wasto }\end{array}$ \\
\hline Disposed & 41347 & 60.68 & & & & & & & & & & & & \\
\hline End Imventory & & 160.48 & 6.80 & 21.1 & & & & & & & & & & \\
\hline \multicolumn{15}{|l|}{ FY 2057} \\
\hline Begis Irventory & & 164.46 & 6.69 & 21.1 & & & & & & & & & & \\
\hline Received & 408.111 & 45220 & & 0.287 & & & & & & & & & & \\
\hline Generated & 10.36 & 35.40 & & & & & & & & & & & & \\
\hline \multicolumn{15}{|l|}{ Reduced } \\
\hline \multicolumn{15}{|l|}{ Transferred } \\
\hline Disposed & 433.47 & 80.66 & & & & & & & & & & & & \\
\hline End Inventory & & 164,48 & 6.60 & 21.36 & & & & & & & & & & \\
\hline \multicolumn{15}{|l|}{ FY 2058} \\
\hline Begin Inventory & & 164.48 & 6.60 & 21.36 & & & & & & & & & & \\
\hline Received & 403.111 & 45229 & 1.0 & 0.257 & & & & & & & & & & \\
\hline Generated & 1036 & 35.43 & 0.01 & 0.02 & & & & & & & & & & \\
\hline \multicolumn{15}{|l|}{ Rectuced } \\
\hline \multicolumn{15}{|l|}{ Transferred } \\
\hline Disposed & 413,47 & 60.66 & & & & & & & & & & & & \\
\hline End Inventory & & 164.48 & 6.69 & 21.62 & & & & & & & & & & \\
\hline \multicolumn{15}{|l|}{ FY 2059} \\
\hline Begin Inventory & & 164.48 & 6.88 & 21.62 & & & & & & & & & & \\
\hline Received & 400111 & 45202 & & 0.257 & & & & & & & & & . & \\
\hline Generated & 70.36 & 35.40 & & & & & & & & & & & & \\
\hline \multicolumn{15}{|l|}{ Reduced } \\
\hline \multicolumn{15}{|l|}{ Transterred } \\
\hline Disposed & 413.47 & 80.66 & & & & & & & & & & & & \\
\hline End Irventory & & 184,48 & 6.69 & 21.88 & & & & & & & & & & \\
\hline \multicolumn{15}{|l|}{ FY 2060} \\
\hline Begin Imventory & & 184.48 & 6.89 & 21.88 & & & & & & & & & & \\
\hline Received & 403.111 & 45220 & & 0.257 & & & & & & & & & & \\
\hline Generated & 10.36 & 35.43 & & & & & & & & & & & & \\
\hline \multicolumn{15}{|l|}{ Recuced } \\
\hline \multicolumn{15}{|l|}{ Transferred } \\
\hline Disposed & 413.47 & 80.80 & & & & & & & & & & & & \\
\hline End Inventory & & 164.48 & 6.80 & 22.15 & & & & & & & & & & \\
\hline \multicolumn{15}{|l|}{ FY 2061} \\
\hline Begin Irwentory & & 164.48 & 6.80 & 22.15 & & & & & & & & & & \\
\hline Received & 403.111 & 45.220 & & 0.257 & & & & & & & & & & \\
\hline
\end{tabular}




\section{SOLID WASTE PROGRAM WBS 1.2.1}

EY 1997 Program Plan

WHC-SP-1114.REV.2

Table 1.3.1-1 Waste Volume Inventory

(cubic meters) (Sheet 15 of 16)

\begin{tabular}{|c|c|c|c|c|c|c|c|c|c|c|c|c|c|c|}
\hline & $\begin{array}{l}\text { UW } \\
\text { (SOL) }\end{array}$ & $\begin{array}{l}\text { UMW } \\
\text { (SOL) }\end{array}$ & TRU & TRUM & HAZ & HWW & $\begin{array}{l}\text { UWW } \\
\text { (LIO) }\end{array}$ & $\mathrm{lum}_{(\mathrm{L} D \mathrm{O})}$ & $\begin{array}{l}\text { Industrial } \\
\text { Whasto } \\
\text { Waler }\end{array}$ & $\begin{array}{l}\text { Trecend } \\
\text { Uifid } \\
\text { Ethuent }\end{array}$ & $\begin{array}{l}\text { Sanitary } \\
\text { (LQ) }\end{array}$ & $\begin{array}{l}\text { Santary } \\
\text { (SOL) }\end{array}$ & Albeeton & $\begin{array}{l}\text { Special } \\
\text { Cees } \\
\text { Waste }\end{array}$ \\
\hline Generated & 1036 & 35.43 & & & & & & & & & & & & \\
\hline \multicolumn{15}{|l|}{ Peduced } \\
\hline \multicolumn{15}{|l|}{ Transferred } \\
\hline Disposed & 413.47 & 80.68 & & & & & & & & & & & & \\
\hline End Inventory & & 164,48 & 6.89 & 2241 & & & & & & & & & & \\
\hline \multicolumn{15}{|l|}{ FY 2062} \\
\hline Begh Inventory & & 164.48 & 6.80 & 2.41 & & & & & & & & & & \\
\hline Received & 402111 & 45.229 & & 0.287 & & & & & & & & & & \\
\hline Generated & 10.36 & 35.43 & & & & & & & & & & & & \\
\hline \multicolumn{15}{|l|}{ Reduced } \\
\hline \multicolumn{15}{|l|}{ Translerred } \\
\hline Disposed & 413.47 & B0.66 & & & & & & & & & & & & \\
\hline End Inventory & & 164.40 & 6.60 & 22.67 & & & & & & & & & & \\
\hline \multicolumn{15}{|l|}{ FY 2063} \\
\hline Begin Inventory & & 164.48 & 6.69 & 22.67 & & & & & & & & & & \\
\hline Received & 403111 & 45220 & 1.0 & 0.257 & & & & & & & & & & \\
\hline Generated & 10.38 & 35.43 & 0.01 & 0.02 & & & & & & & & & & \\
\hline \multicolumn{15}{|l|}{ Reduced } \\
\hline Transferred & & & & - & & & & & & & & & & \\
\hline Disposed & 413.47 & 80.68 & & & & & & & & & & & & \\
\hline End Invertory & & 164.48 & 6.80 & 2293 & & & & & & & & & & \\
\hline \multicolumn{15}{|l|}{ FY 2064} \\
\hline Begin Inventory & & 164.48 & 6.89 & 22.80 & & & & & & & & & & \\
\hline Recoived & 403111 & 45229 & & 0.257 & & & & & & & & & & \\
\hline Generated & 10.36 & 35.43 & & & & & & & & & & & & \\
\hline \multicolumn{15}{|l|}{ Reduced } \\
\hline \multicolumn{15}{|l|}{ Transferred } \\
\hline Disposed & 413.47 & 80.68 & & & & & & & & & & & & \\
\hline End inventory & & 164.48 & 7.81 & 24.21 & & & & & & & & & & \\
\hline \multicolumn{15}{|l|}{ FY 2065} \\
\hline Begin Inventory & & 164.48 & 7.81 & 2421 & & & & . & & & & & & \\
\hline Received & $403: 11$ & 45.220 & & 0.257 & & & & & & & & & & \\
\hline Generated & 10.36 & 35.43 & & & & & & & & & & & & \\
\hline \multicolumn{15}{|l|}{ Reduced } \\
\hline \multicolumn{15}{|l|}{ Transferred } \\
\hline Disposed & 413.47 & 80.66 & & & & & & & & & & & & \\
\hline End Inventory & & 164.48 & 7.81 & 24.47 & & & & & & & & & & \\
\hline
\end{tabular}


SOLID WASTE PROGRAM

WBS 1.2.1

EY 1927 Program Plan

WHC-SP-1114.REV 2

Table 1.3.1-1 Waste Volume Inventory

(cubic meters) (Sheet 16 of 16)

\begin{tabular}{|c|c|c|c|c|c|c|c|c|c|c|c|c|c|c|}
\hline & (SO) & $\operatorname{lumw}_{(\mathrm{sOL})}$ & TAU & TAUM & HAZ & HEW & UW & (1LO) & $\begin{array}{l}\text { Inclustrial } \\
\text { Wauter } \\
\text { Water }\end{array}$ & $\begin{array}{l}\text { Treated } \\
\text { Úpudd } \\
\text { Effluont }\end{array}$ & $\begin{array}{l}\text { Sentitary } \\
\text { (LiO) }\end{array}$ & $\begin{array}{l}\text { Sanhary } \\
\text { (SOL) }\end{array}$ & Astostos & $\begin{array}{l}\text { Special } \\
\text { Case } \\
\text { Wuste }\end{array}$ \\
\hline \multicolumn{15}{|l|}{ FY 2066} \\
\hline Begin imentory & & 164.48 & 7.91 & 24.47 & & & & & & & & & & . \\
\hline Received & 403111 & 452200 & & 0.257 & & & & & & & & & & \\
\hline Generated & 10.36 & 35.43 & & & & & & & & & & & & \\
\hline \multicolumn{15}{|l|}{ Reduced } \\
\hline \multicolumn{15}{|l|}{ Transfenred } \\
\hline Disposed & 413.47 & 80.66 & & & & & & & & & & & & \\
\hline End Imventory & & 164.48 & 7.91 & 24.74 & & & & & & & & & & \\
\hline \multicolumn{15}{|l|}{ FY 2067} \\
\hline Begin Inventory & & 164.48 & 7.91 & 24.74 & & & & & & & & & & \\
\hline Received & 402.111 & 45220 & & 0.257 & & & & & & & & & & \\
\hline Generated & 10.36 & 35.40 & & & & & & & & & & & & \\
\hline \multicolumn{15}{|l|}{ Rectuced } \\
\hline \multicolumn{15}{|l|}{ Transferred } \\
\hline Disposed & 413.47 & 80.68 & & & & & & & & & & & & \\
\hline End Imventory & & 164.48 & 7.91 & 25 & & & & & & & & & & \\
\hline \multicolumn{15}{|l|}{ FY 2068} \\
\hline Begin Inventory & & 164.40 & 7.91 & 25 & & & & & & & & & & \\
\hline Received & 403.111 & 45220 & 1.0 & 0.257 & & & & & & & & & & \\
\hline Generated & 10.36 & 35.43 & 0.01 & 0.08 & & & & & & & & & & \\
\hline \multicolumn{15}{|l|}{ Rectuced } \\
\hline \multicolumn{15}{|l|}{ Transferred } \\
\hline Disposed & 41347 & 80.86 & & & & & & & & & & & & \\
\hline End Inventory & & +64.48 & 7.91 & 25.26 & & & & & & & & & & \\
\hline \multicolumn{15}{|l|}{ FY 2069} \\
\hline Begin Inventory & & 164,48 & 7.91 & 25.26 & & & & & & & & & & \\
\hline Received & 403.111 & 45.220 & & 0.257 & & & & & & & & & & \\
\hline Generated & 10.36 & 35.43 & & & & & & & & & & & & \\
\hline \multicolumn{15}{|l|}{ Rectuced } \\
\hline \multicolumn{15}{|l|}{ Transferred } \\
\hline Disposed & 413,47 & 80.68 & & & & & & & & & & & & \\
\hline End Inventory & & 164.48 & 7.91 & 25.52 & & & & & & & & & & \\
\hline \multicolumn{15}{|l|}{ FY 2070} \\
\hline Begin Inventory & & 164.48 & 7.91 & 25.52 & & & & & & & & & & \\
\hline Recelved & 403.111 & 45220 & & 0.257 & & & & & & & & & & \\
\hline Generated & 10.36 & 36.43 & & & & & & & & & & & & \\
\hline Disposed & 413.47 & $\operatorname{coses}$ & & & & & & & & & & & & \\
\hline End Inventory & & 164.48 & 7.91 & 25.58 & & & & & & & & & & \\
\hline
\end{tabular}




\subsubsection{Nuclear Materials}

Table 1.3.2-1 (not yet developed in theHanford Technical Baseline) contains the nuclear materials (Special Nuclear Materials, Nuclear Fuel, Cesium Capsules Strontium Capsules) inventory and projection data. These data are used to track the nuclear materials through transfer, receipt, storage, and disposition.

\subsubsection{Facilities}

Table 1.3.3-1 contains the facility forecasting data (facility deactivation, D\&D, and closure). The facility data are used to track the facility through acquisition, operations and maintenance, and disposal. The Program schedule and budget reflect the plans for disposition of excess and deactivated facilities.

Table 1.3.3-1 Facility Inventory.

\begin{tabular}{|c|c|c|c|c|c|c|c|}
\hline Complex & Facility & Facility Descriplion & $\begin{array}{l}\text { Acquisition } \\
\text { Project }\end{array}$ & M\&O Project & $\begin{array}{l}\text { Deactivation } \\
\text { Project }\end{array}$ & D\&D Project & Closure Project \\
\hline 200-SWM & 218-E-10 & Burial Ground & & Solid Waste & & & \\
\hline$-\eta-S W M$ & 218-E-12B & Burial Ground & & Solid Waste & & & \\
\hline - & $218-W-3 A$ & Burial Ground & & Solid Waste & & & \\
\hline $200-5 W M$ & $218-W-4 B$ & Burial Ground & & Solid Waste & & & \\
\hline 200-SWM & $218-W-5$ & Burial Ground & & Solid Waste & & & \\
\hline 200-SWM & 218-W-6 & Burial Ground & & Solid Waste & & & \\
\hline 200-SWM & 218-Wo4C & Burial Ground & & Solid Waste & & & \\
\hline $200-5 W M$ & 218-W-3AE & Burial Ground & & Solid Waste & & & \\
\hline 200-SWM & 218-W5252 & ELECTRICAL CONTROL & & Solid Waste & $\begin{array}{l}\text { Real Estate and } \\
\text { Property } \\
\text { Management }\end{array}$ & $\begin{array}{l}\text { Real Estate } \\
\text { and Property } \\
\text { Management }\end{array}$ & $\begin{array}{l}\text { Real Estate and } \\
\text { Property } \\
\text { Management }\end{array}$ \\
\hline 200-SWM & 218-W5252A & ELECTRICAL CONTROL & & Solid Waste & $\begin{array}{l}\text { Real Estate and } \\
\text { Property } \\
\text { Management }\end{array}$ & $\begin{array}{l}\text { Real Estate } \\
\text { and Property } \\
\text { Management }\end{array}$ & $\begin{array}{l}\text { Real Estate and } \\
\text { Property } \\
\text { Management }\end{array}$ \\
\hline 200-SWM & 224- $T$ & TRUSAF & & Solid Waste & & $\begin{array}{l}\text { Environmental } \\
\text { Restoration }\end{array}$ & \\
\hline 200-SWM & $2401 W$ & $\begin{array}{l}\text { PCB/RMW Storage } \\
\text { Building }\end{array}$ & & Solid Waste & & $\begin{array}{l}\text { Environmental } \\
\text { Restoration }\end{array}$ & \\
\hline 200-SWM & $2402 W$ & CWC Storage Building & & Solid Waste & & $\begin{array}{l}\text { Environmental } \\
\text { Restoration }\end{array}$ & \\
\hline 200-SWM & 2402WA & CWC Storage Building & & Solid Waste & & $\begin{array}{l}\text { Environmental } \\
\text { Restoration }\end{array}$ & \\
\hline
\end{tabular}


EY 1997 Program Plan

WHC-SP-1114.REV.2

\begin{tabular}{|c|c|c|c|c|c|c|}
\hline 200-SWM & 2402WB & CWC Storage Building & Solid Waste & & $\begin{array}{l}\text { Environmental } \\
\text { Restoration }\end{array}$ & \\
\hline $200-5 W M$ & $2402 W C$ & CWC Storage Building & Solid Waste & & $\begin{array}{l}\text { Environmental } \\
\text { Restoration }\end{array}$ & \\
\hline 200-SWM & 2402WD & CWC Storage Building & Solid Waste & & $\begin{array}{l}\text { Environmental } \\
\text { Restoration }\end{array}$ & \\
\hline 200-SWM & $2402 W E$ & CWC Storage Building & Solid Waste & & $\begin{array}{l}\text { Environmental } \\
\text { Restoration }\end{array}$ & \\
\hline 200-SWM & $2402 W F$ & CWC Storage Building & Solid Waste & & $\begin{array}{l}\text { Environmental } \\
\text { Restoration }\end{array}$ & \\
\hline 200-SWM & 2402WG & CWC Storage Building & Solid Waste & & $\begin{array}{l}\text { Environmental } \\
\text { Restoration }\end{array}$ & \\
\hline 200-SWM & $2402 W H$ & CWC Storage Building & Solid Waste & & $\begin{array}{l}\text { Environmental } \\
\text { Restoration }\end{array}$ & \\
\hline 200-SWM & $2402 W I$ & CWC Storage Building & Solid Waste & & $\begin{array}{l}\text { Environmental } \\
\text { Restoration }\end{array}$ & \\
\hline 200-SWM & $2402 \mathrm{WJ}$ & CWC Storage Building & Solid Waste & & $\begin{array}{l}\text { Environmental } \\
\text { Restoration }\end{array}$ & \\
\hline 200-SWM & $2402 W K$ & CWC Storage Building & Solid Waste & & $\begin{array}{l}\text { Environmental } \\
\text { Restoration }\end{array}$ & \\
\hline , 0-SWM & $2402 W L$ & CWC Storage Building & Solid Waste & & $\begin{array}{l}\text { Environmental } \\
\text { Restoration }\end{array}$ & \\
\hline 200-SWM & 2403WA & CWC Storage Building & Solid Waste & & $\begin{array}{l}\text { Environmental } \\
\text { Restoration }\end{array}$ & \\
\hline 200-SWM & $2403 W B$ & CWC Storage Building & Solid Waste & & $\begin{array}{l}\text { Environmental } \\
\text { Pestoration }\end{array}$ & \\
\hline 200-SWM & $2403 W C$ & CWC Storage Bullding & Solid Waste & & $\begin{array}{l}\text { Environmental } \\
\text { Restoration }\end{array}$ & \\
\hline 200-SWM & 2403WD & CWC Storage Building & Solid Waste & & $\begin{array}{l}\text { Environmental } \\
\text { Restoration }\end{array}$ & \\
\hline 200-SWM & $2727 W$ & $\begin{array}{l}\text { Sodium Storage } \\
\text { Building }\end{array}$ & Solid Waste & & $\begin{array}{l}\text { Environmental } \\
\text { Restoration }\end{array}$ & \\
\hline 200-SWM & $285 W$ & $\begin{array}{l}\text { RMW Backflow } \\
\text { Preventer }\end{array}$ & Solid Waste & $\begin{array}{l}\text { Real Estate and } \\
\text { Property } \\
\text { Management }\end{array}$ & $\begin{array}{l}\text { Real Estate } \\
\text { and Property } \\
\text { Management }\end{array}$ & $\begin{array}{l}\text { Real Estate and } \\
\text { Property } \\
\text { Management }\end{array}$ \\
\hline 200-SWM & $286 W$ & $\begin{array}{l}\text { RMW BACKFLOW } \\
\text { PREVENTER }\end{array}$ & Solid Waste & $\begin{array}{l}\text { Real Estate and } \\
\text { Property } \\
\text { Management }\end{array}$ & $\begin{array}{l}\text { Real Estate } \\
\text { and Property } \\
\text { Management }\end{array}$ & $\begin{array}{l}\text { Real Estate and } \\
\text { Property } \\
\text { Management }\end{array}$ \\
\hline 200-SWM & 616 & $\begin{array}{l}\text { Non rad Haz. Waste } \\
\text { Facility }\end{array}$ & Solid Waste & & $\begin{array}{l}\text { Environmental } \\
\text { Restoration }\end{array}$ & \\
\hline
\end{tabular}


EY 1997 Program Plan

WHC-SP-1114.REV 2

\begin{tabular}{|c|c|c|c|c|c|c|}
\hline 200-SWM & 6653 & $\begin{array}{l}\text { SAMPLE AND } \\
\text { MONITORING }\end{array}$ & Solid Waste & $\begin{array}{l}\text { Real Estate and } \\
\text { Property } \\
\text { Management }\end{array}$ & $\begin{array}{l}\text { Real Estate } \\
\text { and Property } \\
\text { Management }\end{array}$ & $\begin{array}{l}\text { Real Estate and } \\
\text { Property } \\
\text { Management }\end{array}$ \\
\hline 200-SWM & M0223 & Burial Ground Trailer & Solid Waste & & & \\
\hline 200-SWM & M0278 & Mobile Office & Solid Waste & & & \\
\hline 200-SWM & M0279 & Mobile Office & Solid Waste & & & \\
\hline 200-SWM & M0288 & CwC Staging Trailer & Solid Waste & & & \\
\hline $200-S W M$ & M0289 & TRUSAF Trailer & Solid Waste & & & \\
\hline $200-S W M$ & M0437 & SWM Training Trailer & Solid Waste & & & \\
\hline 200-SWM & M0438 & $\begin{array}{l}\text { Health Physics } \\
\text { Technicians Trailer }\end{array}$ & Solid Waste & & & \\
\hline 200-SWM & M0535 & $\begin{array}{l}\text { Document Processing } \\
\text { and Engineering } \\
\text { Trailer }\end{array}$ & Solid Waste & & & \\
\hline 200-SWM & M0720 & Mobile Office & Solid Waste & & & \\
\hline 200-SWM & M0721 & $\begin{array}{l}\text { Mobile } \\
\text { Office/Operators \& } \\
\text { Operations } \\
\end{array}$ & Solid Waste & & & \\
\hline 200-SWM & M0738 & SWM Trailer & Solid Waste & & & \\
\hline 200-SWM & M0743 & Maintenance Trailer & Solid Waste & & & \\
\hline 200-SWM & M0941 & $\begin{array}{l}\text { TRU Retrieval } \\
\text { Trailer }\end{array}$ & Solid Waste & & & \\
\hline 200-TP & $211 \mathrm{~T}$ & $\begin{array}{l}\text { Chemical } \\
\text { Storage/Handling }\end{array}$ & Solid Waste & & & \\
\hline 200-TP & 211T52 & $\begin{array}{l}\text { Instrumentation } \\
\text { Building }\end{array}$ & Solid Waste & & $\begin{array}{l}\text { Environmental } \\
\text { Restoration }\end{array}$ & \\
\hline 200-TP & $214 \mathrm{~T}$ & $\begin{array}{l}\text { Metal Chemical } \\
\text { Storage }\end{array}$ & Solid Waste & & $\begin{array}{l}\text { Environmental } \\
\text { Restoration }\end{array}$ & \\
\hline 200-TP & $221 \mathrm{~T}$ & T Plant Canyon & Solid Waste & & $\begin{array}{l}\text { Environmental } \\
\text { Restoration }\end{array}$ & \\
\hline 200-TP & 221TA & Fan House & Solid Waste & & $\begin{array}{l}\text { Environmental } \\
\text { Restoration }\end{array}$ & \\
\hline $200-T P$ & 221 TB & $\begin{array}{l}\text { Storage Laundry } \\
\text { Building }\end{array}$ & Solid Waste & & & \\
\hline 200-TP & $225 W A$ & $\begin{array}{l}\text { Treated Effluent } \\
\text { Monitoring }\end{array}$ & Solid Wasle & & $\begin{array}{l}\text { Environmental } \\
\text { Restoration }\end{array}$ & \\
\hline
\end{tabular}


EY 1997 Program Plan

WHC-SP-1114, REV, 2

\begin{tabular}{|c|c|c|c|c|}
\hline 200-TP & $2706 \mathrm{~T}$ & Decon Building & Solid Waste & $\begin{array}{l}\text { Environmental } \\
\text { Restoration }\end{array}$ \\
\hline 200-TP & $2712 T$ & $\begin{array}{l}\text { Electrical } \\
\text { Instrumentation } \\
\text { Building }\end{array}$ & Solid Waste & $\begin{array}{l}\text { Environmental } \\
\text { Restoration }\end{array}$ \\
\hline 200-TP & $2715 T$ & Paint Storage/Materials & Solid Waste & $\begin{array}{l}\text { Environmental } \\
\text { Restoration }\end{array}$ \\
\hline 200-TP & $271 T$ & Office/Main Building & Solid Waste & $\begin{array}{l}\text { Environmental } \\
\text { Restoration }\end{array}$ \\
\hline 200-TP & $2 \pi 7 T$ & Blowdown Building & Solid Waste & $\begin{array}{l}\text { Environmental } \\
\text { Restoration }\end{array}$ \\
\hline 200-TP & $291 T$ & Stack/Exhaust & Solid Waste & $\begin{array}{l}\text { Environmental } \\
\text { Restoration }\end{array}$ \\
\hline 200-TP & $292 \mathrm{~T}$ & Building Laboratory & Solid Waste & $\begin{array}{l}\text { Environmental } \\
\text { Restoration }\end{array}$ \\
\hline $200-\mathrm{TP}$ & $296 \mathrm{~T}$ & $\begin{array}{l}\text { Gaseous Effluent } \\
\text { Release Points }\end{array}$ & Solid Waste & $\begin{array}{l}\text { Environmental } \\
\text { Restoration }\end{array}$ \\
\hline 200-TP & MO371 & Change Trailer & Solid Waste & \\
\hline 200-TP & MO433 & Change Trailer & Solid Waste & \\
\hline 7-TP & MO739 & Change Trailer & Solid Waste & \\
\hline 200-TP & MO892 & Training Trailer & Solid Waste & \\
\hline 200-TP & MO909 & Training Trailer & Solid Waste & \\
\hline 200-TP & Tunnel Trailer & Change Trailer & Solid Waste & \\
\hline 200-WRAP & $2336 \mathrm{~W}$ & WRAP Module 1 & Solid Waste & \\
\hline 200-WRAP & 2404WA & CWC Storage Building & Solid Waste & \\
\hline 200-WRAP & 2404WB & CWC Storage Building & Solid Waste & \\
\hline 200-WAAP & $2404 W C$ & CWC Storage Building & Solid Waste & \\
\hline 200-WRAP & $2470 W$ & $\begin{array}{l}\text { WRAP Maintenance } \\
\text { Trailer }\end{array}$ & Solid Waste & \\
\hline 200-WRAP & $2620 \mathrm{~W}$ & $\begin{array}{l}\text { WRAP Administrative } \\
\text { Trailer }\end{array}$ & Solid Waste & \\
\hline
\end{tabular}

\subsubsection{Infrastructure}

This section contains the forecasting data for infrastructure support. These data are used to ensure the required infrastructure is available when it is needed. Table 1.3.4-1 provides the nominal, best estimate of infrastructure needs. Table 1.3.4-2 provides an estimate of the maximum probable need. The Program schedule and budget reflect the services (infrastructure) that are necessary to achieve the program mission. 


\section{SOLID WASTE PROGRAM \\ WBS 1.2.1}

EY 1997 Program_Plan

WHC-SP-1114,REV 2

Table 1.3.4-1 Infrastructure Requirements - Average Demand.

\begin{tabular}{|c|c|c|c|c|c|c|c|c|c|c|c|}
\hline Infrestructure Type & 1997 & 1980 & 1999 & 2000 & 2001 & 2002 & 2000 & 2004 & 2006 & $2006-2070$ & Units \\
\hline Allocated Land & 20 & 20 & 20 & 20 & 20 & 20 & 20 & 20 & 20 & 20 & requests/yr \\
\hline Analytical Laboratory Services & 540 & 540 & 540 & 540 & 540 & 540 & 540 & 540 & 540 & 540 & sample/yr \\
\hline $\begin{array}{l}\text { Bioassay and Dosimetry } \\
\text { Services }\end{array}$ & 72 & 72 & 72 & 72 & 72 & 72 & 72 & 72 & 72 & 72 & sample/yr \\
\hline Biological Laboratory Services & $\mathbf{2 0}$ & 20 & $\boldsymbol{\infty}$ & $\boldsymbol{\infty}$ & $\mathbf{2 0}$ & 20 & $\boldsymbol{\infty}$ & $\mathbf{2 0}$ & 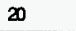 & 20 & sampletyr \\
\hline Cleen Laundy & 117550 & 117550 & 117550 & 117550 & 117550 & 117550 & 117550 & 117550 & 117550 & 117550 & Los $/ y r$ \\
\hline Custodial Services & 90888 & 90888 & 90888 & 90888 & 90888 & 90888 & 90888 & 90888 & 90888 & 90868 & Sq. Ft. \\
\hline Data (HLAN) Transmission & 343 & 343 & 343 & 343 & 343 & 343 & 343 & 343 & 343 & 343 & No. Of Pc's \\
\hline $\begin{array}{l}\text { Development Laboratory } \\
\text { Services }\end{array}$ & 62400 & 62400 & 62400 & 52400 & $\approx 200$ & 62400 & 62400 & 62400 & 62400 & 62400 & $\$ / y r$ \\
\hline Electricity & 416 & 416 & 416 & 416 & 416 & 416 & 416 & 416 & 416 & 416 & MW-hr/yr \\
\hline Fab Shop Services & 1070 & 1070 & 1070 & 1070 & 1070 & 1070 & 1070 & 1070 & 1070 & 1070 & Labor -hr/yr \\
\hline Guaranteed Ride Home & 73 & 73 & 73 & 73 & 73 & 73 & 73 & 73 & 73 & 73 & passengers/yr \\
\hline $\begin{array}{l}\text { Hanford Road Sys. Heawy } \\
\text { Traffic }\end{array}$ & 525 & 525 & 525 & 525 & 525 & 525 & 525 & 525 & 525 & 525 & Truck loads/yr \\
\hline Heay Equipment & 1505 & 1505 & 1505 & 1505 & 1505 & 1506 & 1505 & 1506 & 1505 & 1505 & equip-days/yr \\
\hline Heavy Trucks & 4186 & 4186 & 4186 & 4186 & 4186 & 4186 & 4186 & 4186 & 4186 & 4186 & Vehicle -hr/yr \\
\hline In-Field Laboratory Services & 11 & 11 & 11 & 11 & 11 & 11 & 11 & 11 & 11 & 11 & sampletyr \\
\hline Industrial Hygiene Services & $\mathbf{z 0}$ & 20 & 20 & $\mathbf{2 0}$ & $\boldsymbol{x}$ & 20 & $\mathbf{n}$ & 20 & $\mathbf{2 0}$ & 20 & sample/yr \\
\hline Lifting (Cranes) & 670 & 670 & 670 & 670 & 670 & 670 & 670 & 670 & 670 & 670 & Crane dayshr \\
\hline $\begin{array}{l}\text { Non-rad Standards } \\
\text { (Calibrations) }\end{array}$ & 40 & 40 & 40 & 40 & 40 & 40 & 40 & 40 & 40 & 40 & Calib/yr \\
\hline Office Space (Program Owned) & 49088 & 49089 & 49088 & 49088 & 49088 & 49088 & 49088 & 49088 & 49088 & 49088 & Sq. Ft. \\
\hline Pager Senvice & 208 & 208 & 208 & 208 & 208 & 208 & 208 & 208 & 208 & 200 & No. Of pagers \\
\hline Potable Water & 3197 & 3197 & 3197 & 3197 & 3197 & 3197 & 3197 & 3197 & 3197 & 3197 & mgal/yr \\
\hline $\begin{array}{l}\text { Radioactive Standards } \\
\text { (Calibrations) }\end{array}$ & 778 & 778 & 778 & $78 B$ & 778 & $\pi 8$ & 778 & $\pi 78$ & 778 & 778 & Calib/yr \\
\hline Rail Transportation & 6 & 6 & 6 & 6 & 6 & 6 & 6 & 6 & 6 & 6 & Rail cars/yr \\
\hline Sedans/Light Trucks & 14 & 14 & 14 & 14 & 14 & 14 & 14 & 14 & 14 & 14 & No. Of vohicles \\
\hline Taxi Service & 821 & 821 & 821 & 821 & 821 & 621 & 821 & 821 & 821 & 821 & passengers/yr \\
\hline Video Communication & 2245 & 2245 & 2245 & 2245 & 2245 & 2245 & 2245 & 2245 & 2245 & 2245 & hr/yr \\
\hline
\end{tabular}


Table 1.3.4-2 Infrastructure Requirements - Peak Demand.

\begin{tabular}{|c|c|c|c|c|c|c|c|c|c|c|c|}
\hline Intrastructure Type & 1997 & 1998 & 1999 & 2000 & 2001 & 2002 & 2003 & 2004 & 2005 & $\begin{array}{l}2006- \\
2070\end{array}$ & Units \\
\hline Allocated Land & 24.0 & 24.0 & 2.40 & 240 & 24.0 & 24.0 & 24.0 & 24.0 & 240 & 24.0 & requeasts/yr \\
\hline Analytical Laboratory Services & 900 & 900 & 900 & 900 & 900 & 900 & 900 & 900 & 900 & 900 & sample/yr \\
\hline $\begin{array}{l}\text { Bioassay and Dosimetry } \\
\text { Services }\end{array}$ & 72 & 72 & 72 & 72 & 72 & 72 & 72 & 72 & 72 & 72 & sample/yr \\
\hline Biological Laboratory Services & 72 & 72 & 72 & 72 & 72 & 72 & 72 & 72 & 72 & 72 & sample/yr \\
\hline Clean Laundy & 117550 & 117550 & 117550 & 117550 & 117550 & 117550 & 117550 & 117550 & 117550 & 117550 & Losfyr \\
\hline Custodial Services & 90888 & 90888 & 90888 & 90888 & 90888 & 90888 & 90088 & 90089 & 90888 & 90888 & Sq. Ft. \\
\hline Data (HLAN) Transmission & 343 & 343 & 343 & 343 & 343 & 343 & 343 & 343 & 343 & 343 & No. Of Pc's \\
\hline $\begin{array}{l}\text { Development Laboratory } \\
\text { Services }\end{array}$ & 124800 & 124800 & 124800 & 124800 & 124800 & 124800 & 124800 & 124800 & 124800 & 124800 & $\$ / y r$ \\
\hline Electricity & 3644.16 & 3644.16 & 3644.16 & 3644.16 & 3644.16 & 3644.16 & 3644.16 & 3644.16 & 3644.16 & 3644.16 & MW-hr/yr \\
\hline Fab Shop Services & 1390 & 1380 & 1380 & 1380 & 1380 & 1380 & 1390 & 1380 & 1380 & 1380 & Labor -hr/yr \\
\hline Guaranteed Ride Home & 3285 & 3285 & 3285 & 3285 & 3285 & 3285 & 3285 & 3285 & 3285 & 3205 & passengers/yr \\
\hline $\begin{array}{l}\text { Hanford Road Sys. Heavy } \\
\text { Tratfic }\end{array}$ & 9125 & 9125 & 9125 & 9125 & 9125 & 9125 & 9125 & 9125 & 9125 & 9125 & Truck loads/yr \\
\hline Heavy Equipment & 1505 & 1505 & 1505 & 1505 & 1505 & 1505 & 1505 & 1505 & 1505 & 1505 & equip-days/yr \\
\hline Heavy Trucks & 13505 & 13505 & 13505 & 13505 & 13505 & 13505 & 13505 & 13505 & 13506 & 13505 & Vehicle -hr/yr \\
\hline In-Field Laboratory Services & 60 & 60 & 60 & 60 & 6 & 6 & 6 & 60 & 6 & 60 & sample/yr \\
\hline Industrial Hygiene Services & 72 & 72 & 72 & 72 & 72 & 72 & 72 & 72 & 72 & 72 & sample/yr \\
\hline Lifting (Cranes) & 670 & 670 & 670 & 670 & 670 & 670 & 670 & 670 & 670 & 670 & Crane dayshyr \\
\hline $\begin{array}{l}\text { Non-rad Standards } \\
\text { (Calibrations) }\end{array}$ & 360 & 360 & 360 & 360 & 360 & 360 & 360 & 360 & 360 & 360 & Calib./yr \\
\hline $\begin{array}{l}\text { Office Space (Program } \\
\text { Owned) }\end{array}$ & 49088 & 49088 & 49088 & 49088 & 49088 & 49088 & 49088 & 49088 & 49088 & 49088 & Sq. Ft. \\
\hline Pager Senvice & 2008 & 208 & 208 & 208 & 208 & 208 & 208 & 208 & 208 & 208 & No. Of pagers \\
\hline Potable Water & 3554.11 & 3554.11 & 3554.11 & 3554.11 & 3554.11 & 3554.11 & 3554.11 & 3554.11 & 3554.11 & 3554.11 & mgal/yr \\
\hline $\begin{array}{l}\text { Radioactive Standards } \\
\text { (Calibrations) }\end{array}$ & 852 & 852 & 852 & 852 & 852 & 852 & 852 & 852 & 652 & 852 & Calib./yr \\
\hline Rail Transportation & 6 & 6 & 6 & 6 & 6 & 6 & 6 & 6 & 6 & 6 & Rail cars/yr \\
\hline Sedans/Light Trucks & 14 & 14 & 14 & 14 & 14 & 14 & 14 & 14 & 14 & 14 & $\begin{array}{l}\text { No. Of } \\
\text { vehicles }\end{array}$ \\
\hline Taxi Service & 5110 & 5110 & 5110 & 5110 & $\mathbf{5 1 1 0}$ & 5110 & $\mathbf{5 1 1 0}$ & 5110 & 5110 & 5110 & passengarshyr \\
\hline Video Communication & 2245 & 2245 & 2245 & 2245 & 2245 & 2245 & 2245 & 2245 & 2245 & 2245 & $\mathrm{hr} / \mathrm{yr}$ \\
\hline
\end{tabular}




\subsection{DRIVERS}

This section identifies the documents that are sources of program requirements, including Key and Regulatory Drivers.

\subsubsection{Key Drivers}

Table 1.4.1-1 lists the source documents that tend to drive the program mission (e.g., Mission Direction Document, Tri-Party Agreement).

Table 1.4.1-1 Key Drivers.

\begin{tabular}{||l|l||}
\hline Name & Title \\
\hline DOE Order 5633.3B & Control and Accountability of Nuclear Materials \\
\hline DOE/RL-96-14 & Updated Draft Mission Direction Document, June 1996 \\
\hline Tri-Party Agreement & Hanford Federal Facility Agreement and Consent Order:89-10, Rev. 1 \\
\hline
\end{tabular}

\subsubsection{Other Drivers}

All documents and applicable requirements to the Solid Waste Division are listed in WHC-IP-1120, REV. 4, "Standards/Requirements Identification Document (S/RID)."

\subsection{FUNCTIONAL DEFINITIONS}

This section contains the Hanford Site Technical Baseline functions that are assigned to the program. It describes the program work in terms consistent with the Hanford Site Technical Baseline Database System.

\subsubsection{Manage Solid Waste Remediation System}

Maintain a safe and compliant solid waste operational environment by continually assessing and maintaining the solid waste operational environment in a safe and compliant condition.

Maintain safe and compliant solid waste system, equipment and structures. Continually assess and maintain the solid waste facility systems, equipment, and structures, and their operation in a safe condition. Maintain a qualified solid waste staff, and maintain required solid waste facility and operating documentation. 
Maintain safe and compliant solid waste documentation [Safety Analysis Reports (SAR), Interim Operational Safety Requirements (OSR), Waste Acceptance Criteria, Performance Assessments (PAs), etc.]. Maintain required solid waste facility and operating documentation related to safe and compliant operations.

Provide program control including scheduling, financial analysis, change control preparation, organization financia/business management, programmatic organizational financial/schedule analysis and program/performing organization business reporting activities. Scheduling activities include fiscal year and outyear development, cost account monitoring, and change control tasks and reporting. Financial activities include fiscal year budget development and outyear requirements planning, cost account monitoring and change control tasks including variance analysis reports and estimate at completion report preparation.

Dispose solid waste. Schedule solid waste package disposal and prepare waste transport package and disposal documentation. Provide final inspection of the solid waste package, load, and place in disposal site.

Through a systems engineering approach develop and maintain baseline documentation including defining the technical baseline of the program and interfaces with other site programs, develop alternatives and trade studies for materials requiring a path-definition for management and tracking of solid waste through the Solid Waste Information Tracking System (SWITS).

Prepare solid waste disposition specifications. Generate specifications for solid waste operations include treatment, packaging, certification, storage, shipping, disposition, and archiving samples and records.

Assess solid waste characterization information. Evaluate the solid waste characterization information consisting of generator request information, process knowledge information, and waste sample characterization data, against solid waste disposition requirements and provide validation of meeting the disposition requirements.

Assess solid waste treatment, storage, and disposal (TSD) capability needs and resources based on required versus design throughput and capacities.

Issue environmental documentation for planned facilities and operations, in accordance with Solid Waste Program schedule.

Obtain and maintain required permits for operation and closure of solid waste facilities.

Issue and maintain an integrated "Solid Waste Program Multi-Year Work Plan." 


\section{SOLID WASTE PROGRAM \\ WBS 1.2.1}

EY 1997 Program Plan

WHC-SP-1114, REY, 2

\section{Maintain "Solid Waste Technical Baseline Description."}

Issue and maintain "Interface Control Documents" with all programmatic interfaces.

Establish criteria for common aspects of safety analyses and issue integrated safety documentation for planned facilities and operations.

Verify LLW and LLMW packages to confirm generator characterizations.

\subsubsection{Acquire Solid Waste Remediation System}

Not applicable to this program.

\subsubsection{Operate and Maintain Solid Waste Remediation System}

1

Table 1.5.3-1 Operate and Maintain Solid Waste Remediation System Functions.

\begin{tabular}{|c|c|}
\hline Number & Name/Description \\
\hline 4.3 & $\begin{array}{l}\text { Bemedy solld Weste } \\
\text { This function safely stores solid waste currently contained in burial grounds and above ground storage } \\
\text { facilities pending final disposition. It retrieves, treats, and disposes of buried wastes and solid waste } \\
\text { materials generated during past missions, solid wastes generated during the cleanup mission, solid waste } \\
\text { received from offsite generators, and the solid components of hazardous liquids. }\end{array}$ \\
\hline 4.3.1 & $\begin{array}{l}\text { Maintain Solid Waste Safety and Compliance Envelope } \\
\text { Continually assess and maintain the waste form and the facility structures and operations in a safe and } \\
\text { compliant condition. Includes maintaining a qualified facility stafi, and maintaining required } \\
\text { safety/compliance documentation. }\end{array}$ \\
\hline 4.3.2 & $\begin{array}{l}\text { Receive Solld Waste } \\
\text { Retrieve legacy solid waste (1970-1989) and receive solid waste generated during cleanup operations and } \\
\text { solid waste from other missions. }\end{array}$ \\
\hline 4.3.3 & $\begin{array}{l}\text { Characterize Solid. Waste } \\
\text { Identify, inventory, and characterize the waste by reviewing the process operations (current and } \\
\text { historical), by reviewing new waste information and by sampling and analy zing the waste inventory. } \\
\text { Assess the current condition of the waste. Archive the results of these investigations. }\end{array}$ \\
\hline 4.3 .4 & $\begin{array}{l}\text { Determine solid Waste Dlsposition Requirements } \\
\text { Compile and sort imposed requirements, define requirements that result from proposed solutions and } \\
\text { required capabilities, and assess constraints under which the function must operate. }\end{array}$ \\
\hline 4.3 .5 & $\begin{array}{l}\text { Disposition Solid Waste } \\
\text { Package and certify the solid waste, store or ship as needed, treat andor immobilize it, and accomplish } \\
\text { final disposition. }\end{array}$ \\
\hline
\end{tabular}




\subsubsection{Dispose of Solid Waste Remediation System}

Not yet developed in Hanford Site Technical Baseline.

\subsection{PROGRAM LIFE CYCLE REQUIREMENTS}

This section contains the requirements for each program life cycle phase including the program management requirements.

\subsubsection{Manage Solid Waste Remediation System}

Not yet developed in Hanford Site Technical Baseline.

\subsubsection{Acquire Solid Waste Remediation System}

Not applicable to this program.

\subsubsection{Operate and Maintain Solid Waste Remediation System}

Table 1.6.3-1 Operate and Maintain Solid Waste Remediation System

\begin{tabular}{|c|c|}
\hline 1 & L96MYPP(9D) Suspect TRU solid waste shall be retrieved and characterized. \\
\hline 2 & $\begin{array}{l}\text { SW 96MYPP (20D) Contaminated equipment storage and storage of spent fuel elements shall be } \\
\text { provided at T Plant. }\end{array}$ \\
\hline 3 & SW 96MYPP(15D) Solid waste shall be shipped and waste transfer verified. \\
\hline 4 & SW 96MYPP (8D) Required solid waste facility and operating documentation shall be maintained. \\
\hline 5 & $\begin{array}{l}\text { SW 96MYPP(ID) Solid Waste minimization goals shall be met by developing repackaging techniques, } \\
\text { planning, and recycling. }\end{array}$ \\
\hline 6 & $\begin{array}{l}\text { SW 96MYPP (21D) Final disposition of solid waste shall be accomplished by treatment/storage } \\
\text { and/or immobilization for packaging; certification; packaging; and storage and/or shipment. }\end{array}$ \\
\hline 7 & SW 96MYPP(7D) Safe and compliant solid waste documentation shall be maintained. \\
\hline 8 & $\begin{array}{l}\text { SW 96MYPP (19D) Solid waste shall be repackaged to ensure that the solid waste is in a container } \\
\text { suitable for the waste type. }\end{array}$ \\
\hline 9 & $\begin{array}{l}\text { SW 96MYPP (2D) Solid Waste operations shall be within requirements established by the U.S. } \\
\text { Environmental Protection Agency (EPA) Resources Conservation and Recovery Act (RCRA) and State of } \\
\text { Washington Department of Ecology (Ecology) and the Department of Health (DOH) for treatment, } \\
\text { storage, disposal, and decontamination of waste. }\end{array}$ \\
\hline
\end{tabular}




\begin{tabular}{|c|c|}
\hline 10 & $\begin{array}{l}\text { SW 96MYPP(14D)Newly generated solid waste generated during Hanford Cleanup mission operations } \\
\text { and from other missions shall be received for treatment, storage, and disposal. }\end{array}$ \\
\hline 11 & $\begin{array}{l}\text { SW 96MYPP (17D) High dose rate waste and contaminated equipment shall be processed in the } 221 \text { - } \\
T \text { canyon to meet applicable standards for disposal, storage, re-use, or free release. }\end{array}$ \\
\hline 12 & $\begin{array}{l}\text { SW 96MYPP(25D) Archival storage and retrieval operations for the analyzed solid waste process } \\
\text { sample analysis data shall be provided. }\end{array}$ \\
\hline 13 & $\begin{array}{l}\text { SW 96MYPP_(13D) Solid waste shall be stored in a safe and compliant manner prior to shipment to } \\
\text { an approved treatment and/or disposal site. }\end{array}$ \\
\hline 14 & $\begin{array}{l}\text { SW 96MYPP (3D) } \\
\text { Solid Waste operations shall utilize the best demonstrated available technologies shall be utilized. }\end{array}$ \\
\hline 15 & SW 96MYPP (24D) Solid waste shall be certified and verified. \\
\hline 16 & $\begin{array}{l}\text { SW 96MYPP(10D) Solid waste shall be treated to convert the waste to an acceptable form for final } \\
\text { disposition. }\end{array}$ \\
\hline 17 & $\begin{array}{l}\text { SW 96MYPP (12D) Contents of "unknown" or "backlog" wastes shall be inspected to provide } \\
\text { segregation and ensure compliant packaging of waste. }\end{array}$ \\
\hline 18 & $\begin{array}{l}\text { SW 96MYPP(22D) Waste received from generators shall be identified, inventoried, and } \\
\text { characterized, the current condition of the waste shall be assessed, and the results archived. }\end{array}$ \\
\hline
\end{tabular}

\begin{tabular}{|c|c|}
\hline 19 & $\begin{array}{l}96 M Y P P(23 D) \text { Solid waste characterization information shall be assessed and validation that the disposition } \\
\text { requirements were met provided. }\end{array}$ \\
\hline 20 & 96MYPP(11D) container contents of newly generated waste shall be inspected to verily waste contents. \\
\hline 21 & SW 96MYPP(4D) A safe and compliant solid waste operational environment shall be maintained. \\
\hline 22 & SW 96MYPP(5D) Safe and compliant solid waste systems. equipment and structures shall be maintained. \\
\hline 23 & SW 96MYPP(16D) Solid waste shall be disposed of by placement in a disposal site. \\
\hline 24 & $\begin{array}{l}\text { SW 96MYPP(6D) A qualified solid waste staff, and required solid waste facility and operating documentation shall be } \\
\text { maintained. }\end{array}$ \\
\hline 25 & $\begin{array}{l}\text { SW 96MYPP(18D) Incoming wastes shall be received, stored, contained, decontaminated, and repackaged for } \\
\text { reduction in radiation fields and recategorization from nixed to non-mixed and/or Greater Than Category } 3 \text { low level to } \\
\text { Category } 1 \text { or } 3 \text { low-level or, if possible, to levels making the waste a releasable nonradioactive) material. }\end{array}$ \\
\hline 26 & $\begin{array}{l}\text { MDD(229) Conduct operations and maintenance of the Non-radioactive Waste Storage Facility (Building 616) in cold } \\
\text { standby mode. }\end{array}$ \\
\hline 27 & MDD(186) Maintain Liquid Waste Tank Car: provide maintenance, repairs, testing and certification. \\
\hline 28 & MDD(187) Maintain and operate WRAP I facility. \\
\hline 29 & MDD(188) Provide surveillance, maintenance and monitoring of contaminated equipment stored in the 221-T Canyon. \\
\hline 30 & $\begin{array}{l}\text { MDD(189) provide decontamination and maintenance services in support of essential safety operations at the } \\
\text { 2706-TA Building: equipment decontamination: LW and RMW packaging. }\end{array}$ \\
\hline
\end{tabular}




\begin{tabular}{|c|c|}
\hline 31 & $\begin{array}{l}\text { MDD(190) conduct operations, surveillance, and maintenance of the 221-T TRU Storage and Assay Facility (TRUSAF), } \\
\text { the Central Waste Complex (CWC) storage facilities. }\end{array}$ \\
\hline 32 & MDD(191) Conduct operations. surveillance and maintenance of the Low Level Waste burial Grounds (LLBG). \\
\hline 33 & MDD(78) Retrievably stored TRU waste retrieved, processed. shipped off-site to WIPP. \\
\hline 34 & $\begin{array}{l}\text { MDD(79) Low-level and low-level mixed waste from on-site and off-site sources (including PNNL special case wastes) } \\
\text { will continue to be disposed of in the } 200 \text { Area. }\end{array}$ \\
\hline 35 & MDD(77) Off-site TRU shipments to Hanford for interim storage will be reinstaled. \\
\hline 36 & $\begin{array}{l}1995 \text { HMP.Yol. 1. para, } 3,3,3.2(02 \mathrm{D}) \text { The } 200 \text { Areas disposal systems shall use barriers to limit infiltration by } \\
\text { rainwater. }\end{array}$ \\
\hline 37 & $\begin{array}{l}1995 \text { HMP.Vol 1. Dara, 3.3.3.2(03D) Site-specific CERCLA RODs or RCRA past-practices corrective measures } \\
\text { shall control the actual cleanup of unsegregated buried solid waste. }\end{array}$ \\
\hline 39 & $\begin{array}{l}1995 \text { HMP.Vol. 1, para, 3.3.3.2(13D) Newly generated solid waste shall be segregated by waste type with LLW } \\
\text { disposed of onsite, mixed waste treated and disposed of onsite, and TRU waste treated and disposed of offsite. }\end{array}$ \\
\hline 40 & $\begin{array}{l}1995 \text { HMP Vol. L. Dars. 3.3.3.2(14D) Disposal of retrievably stored and newly generated TRU solid waste shall } \\
\text { be in accordance with the Hanford Defense Waste Environmental Impact Statement (HDW-EIS), DOE/EIS-113. }\end{array}$ \\
\hline
\end{tabular}

\subsubsection{Remedy Solid Waste Remediation System}

Table 1.6.3.1-1 Remedy Solid Waste Remediation System.

\begin{tabular}{||l|l||}
\hline 1 & $\begin{array}{l}\text { Offsite Dlsposition of Transuranic Wastes } \\
\text { Transuranic wastes that have been approved for offsite disposition shall be packaged in accordance with the waste } \\
\text { acceptance criteria of the offsite waste disposal facilities and shipped in accordance with transportation requirements } \\
\text { to the offsite waste disposal facilities. }\end{array}$ \\
\hline 2 & $\begin{array}{l}\text { Offsite Disposition of Hazardous and Mixed Wastes } \\
\text { Hazardous and mixed wastes that have been approved for offsite disposition shall be packaged in accordance with } \\
\text { the waste acceptance criteria of the offsite waste disposal facilities and shipped in accordance with transportation } \\
\text { requirements to the offsite waste disposal facilities. }\end{array}$ \\
\hline 3 & $\begin{array}{l}\text { Remedy Solld Waste Mission } \\
\text { Buried waste, solid waste materials generated during past missions and solid waste generated during the cleanup } \\
\text { mission shall be dispositioned. }\end{array}$ \\
\hline
\end{tabular}

\subsubsection{Dispose of Solid Waste Remediation System}

Not yet developed in Hanford Site Technical Baseline. 


\subsection{PROGRAM ISSSUES AND ASSUMPTIONS}

This section contains the issues that must be resolved by the program. These include program-specific issues, as well as the site-level issues that have been assigned to the program for resolution. It also contains the assumptions that are used as a basis for the development of program plans until the issues are formally resolved with records of decision (see Table 1.7-1).

Additionally, various Program Constraints are applicable to the MYWP. The following apply:

Uncertainties in waste data impact effective planning for treatment. storage, disposal

Volume and characterization data for some waste types are uncertain and add to planning risks. These uncertainties affect waste identified for disposition by the Solid Waste Program. Future National Environmental Policy Act (NEPA) and Federal Facility Compliance Act (FFCAct) decisions may impact the future volumes and types of waste received.

Many factors contribute to uncertainties in waste data. Data on older waste (circa post-1970 stored TRU waste) are less detailed and less specific than data on new waste. Volume forecasting contains uncertain aspects due to funding availability, programmatic impacts, and decisions awaiting approval through the NEPA process and FFCAct planning.

\section{Insufficient TRU Analytical Laboratory Support at Hanford}

Emerging requirements regarding WRAP process support and WIPP waste acceptance criteria have outpaced available analytical capabilities, both within the DOE complex and in the commercial sector. Specialized laboratory facilities are required to analyze TRU contaminated samples to environmental protocols for waste processing and shipment to the WIPP. Facilities at the Hanford site and capacity on a national scale (commercial and DOE) is extremely limited. The Hanford site needs these services to support the WRAP facility. Commercial laboratories may provide interim services for lower-activity services $(<200 \mathrm{nCi} / \mathrm{gm})$. Hanford Analytical Labs are working with offsite DOE facilities to obtain services for $>200 \mathrm{nCi} / \mathrm{gm}$ ) samples.

\section{Plant Related Issues}

Since line item funding is not provided in FY 1997 for T Plant ventilation upgrade, project C-077, then there will be a year-for-year slip in the completion date of June, 2001, resulting in risk in personnel working conditions related to temperature control, and work delays. 


\section{TRU Head Gas Sampling}

Transportation to WIPP in TRUPACT containers requires that headgas samples be taken. The headgas sampling capability was originally in Project W-112, but was removed when TRU retrieval and WRAP TRU Processing were deleted from the funding baseline. There is no current planning in place to support this effort. Funding for this will be addressed by change requewst early in FY1997 along with responses to a WIPP certification audit performed at the end of FY1997.

\section{Sodium Storage}

Solid Waste Operations maintains indefinite storage of radioactively contaminated metallic sodium material (Sodium Reactor Experiment and Hallam Sodium). The DOE plan to shutdown PUREX operations has resulted in an indeterminate delay in the use of converted sodium hydroxide. Alternate users such as the Savannah River Site were evaluated, but not found viable. Currently, it remains in non-RCRA storage classified as material. Solid Waste is awaiting a decision from TWRS (planned for FY 1998) regarding the potential use of the sodium in the privatized vitrification process. If no use is identified, this material will be designated as RCRA mixed waste. Once sodium material has been declared as waste, it must be placed in RCRA compliant storage and/or properly disposed. At this time, construction of storage facilities for tanks of Hallam sodium has not been initiated while commercial treatment is pursued. No funding currently allocated to Sodium Treatment.

\section{WIPP Operation}

The current projected capacity of the CWC is based on WIPP receiving certified TRU waste from Hanford.

\section{Initiation of TRU Waste Shipments to WIPP}

Preparatory work for shipment of TRU waste to WIPP was truncated in FY1995 and recently restarted as reflected in this MYWP. A solid basis of estimate has been performed which defines requirements for startup of the WRAP TRU line, however, a comprehensive plan for initiating shipments to WIPP (including activities such as head gas sampling originally provided by project W-112 and TRU lab capability) has not yet been completed. Additionally, a WIPP certification team recently completed an audit of WRAP and the results have not yet been published. A change request will be submitted early in FY1997 identifying all workscope required to initiate WIPP shipment on 1/7/98. If WIPP continues to be delayed, additional RCRA-compliant mixed waste storage-capacity will be required. 


\section{SOLID WASTE PROGRAM \\ WBS 1.2.1}

EY 1997 Program Plan

WHC-SP-1114.REV. 2

\section{Facility Closure}

Institutional control and the future mission of the Hanford site remain an issue concerning the Low Level Waste Burial Ground closure. Due to the proximity of facilities in the 200 Areas, utilization of land for utilities, access roads, and other adjacent activities will impact the current closure schedule.

\section{Project W-113 TRU Retrieval}

Project W-113 planning does not reflect TPA M-91-04. A change request will be intiated in FY1997 to adjust outyear schedules and budget to meet the TPA M-91 milestones.

Table 1.7-1 Program Issues and Assumptions.

\begin{tabular}{|c|c|c|c|}
\hline & Issue & Interim Decision & Champion \\
\hline 1 & $\begin{array}{l}\text { Sodium Dispealitien Potential use as a neutralization agent for } \\
\text { tank waste is projected to be beyond } 2015 \text {. }\end{array}$ & Interim store in the 400 Area and CWC until eventual use by TWR. & Mecca \\
\hline 2 & $\begin{array}{l}\text { TRU Yheste Dispositton The projected volume of TRU waste at } \\
\text { Haniord exceeds the volume allotted for Hanford waste at WIPP. }\end{array}$ & $\begin{array}{l}\text { DOE Order 5820.2A, Chapter 11, establishes the DOE pelicy for TRU } \\
\text { waste. a "Transuranic waste shail be certified to be in compliance } \\
\text { with the Waste Isolation Pilot Plant-Waste Acceptance Criteria, } \\
\text { placed in interim storage (if required), and sent to the Waste } \\
\text { Isolation Pilot Plant." DoE Order 5820.2A, Chapter 11, establishes } \\
\text { the DOE policy for. TRU waste. a. "Transuranic wastes shall be } \\
\text { certified to be in compllance with the Waste isolation Pllot Plant- } \\
\text { Waste Acceptance Criteria, placed in interim storage (if required). } \\
\text { and sent to the Waste Isolation Pilot Plant." }\end{array}$ & Guercia \\
\hline 3 & 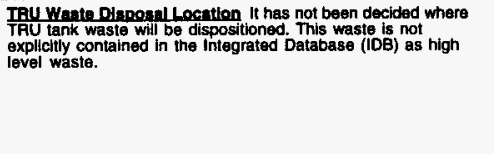 & 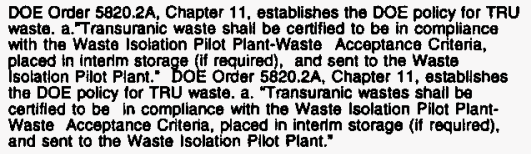 & Kinzer \\
\hline 5 & $\begin{array}{l}\text { Land Une Pian } 2000 \text { The Interim end state for the } 200 \text { Areas has } \\
\text { not been defined. }\end{array}$ & $\begin{array}{l}\text { The } 200 \text { Area and central plateau will be used for the management } \\
\text { of nuclear materiais and the collectlon and dispossal of waste } \\
\text { materials that remain on site and for other related and compatible } \\
\text { uses. Claanup levels and disposal standards will be established in } \\
\text { the CERCLA and RCFA pernit modifications. Soil sites remediated } \\
\text { consistent with ROD cleanup standards. }\end{array}$ & McClain \\
\hline 6 & $\begin{array}{l}\text { Soll.Cleanup Criterta Criteria for contaminated soil cleanup } \\
\text { have not boen integrated. }\end{array}$ & Soil sites remediated consistent with ROD cleanup standards. & McClain \\
\hline 7 & $\begin{array}{l}\text { End State for Canyon Facllition The end state for the canyon } \\
\text { facifities has not been clearly defined. }\end{array}$ & $\begin{array}{l}\text { Canyon tacilities will be partially demolished with material disposed } \\
\text { onsite. }\end{array}$ & McClain \\
\hline
\end{tabular}




$$
\begin{gathered}
\text { Section } 2.0 \\
\text { Work Breskdoun } \\
\text { Structure }
\end{gathered}
$$




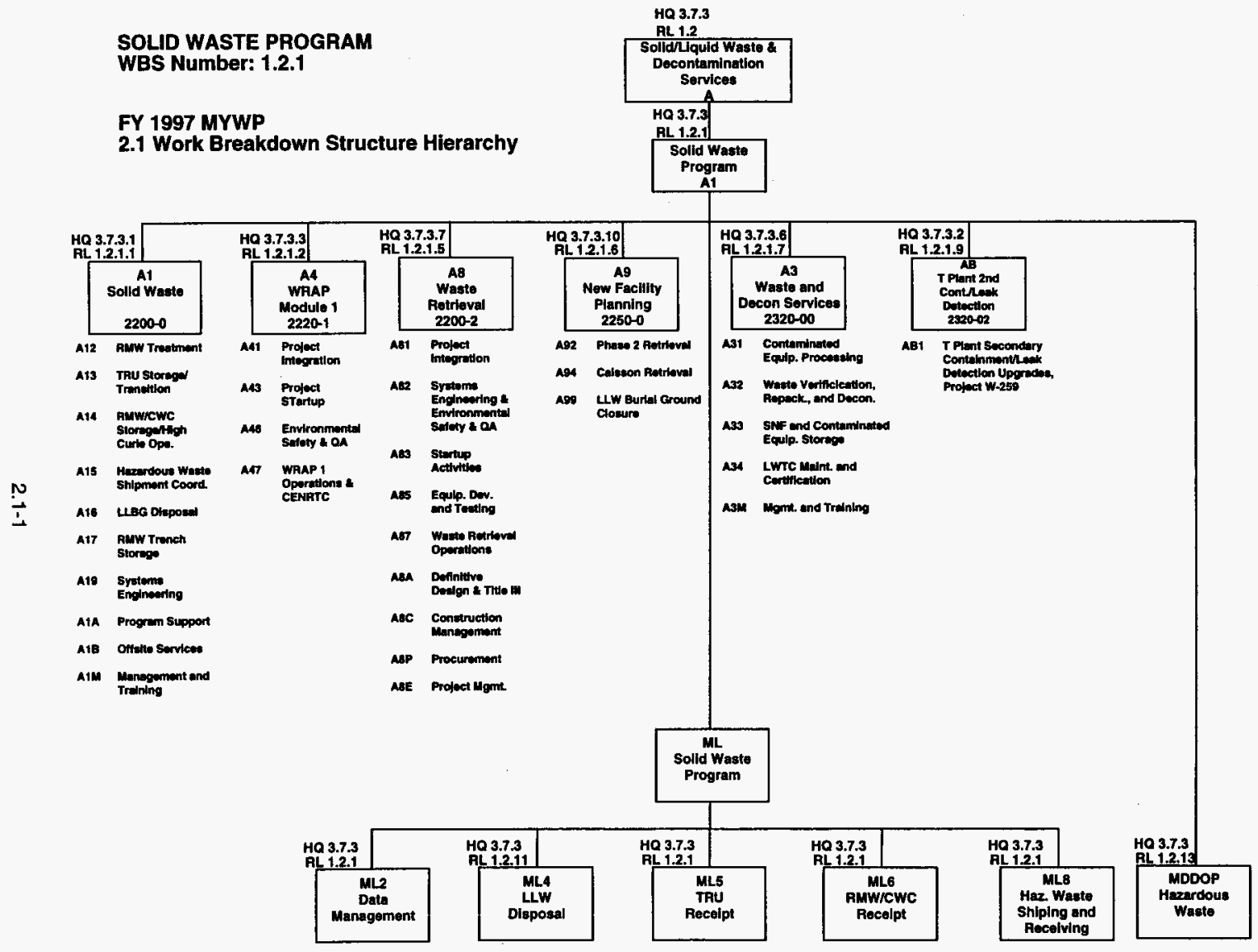




\begin{tabular}{|l|l|l||}
\hline \multicolumn{3}{|c|}{ WORK BREAKOOWN STRUCTURE DIOTIONARY } \\
\hline $\begin{array}{l}\text { 1 PROJECT TITLE/PARTICIPANT } \\
\text { Solid Waste Program }\end{array}$ & $\begin{array}{l}2 \text { DATE } \\
08 / 31 / 96\end{array}$ & $\begin{array}{l}3 \text { IDENTIFICATION NO. } \\
3.7 .3 .1\end{array}$ \\
\hline 4 WBS ELEMENT CODE & $\begin{array}{l}5 \text { WBS ELEMENT TITLE } \\
1.2 .1 .1\end{array}$ & RMW Treatment \\
\hline $\begin{array}{l}\text { 6 INDEX LINENO. } \\
\text { 1A12 }\end{array}$ & $\begin{array}{l}7 \text { REVISION NO. AND } \\
\text { AUTHORIZATION }\end{array}$ & $\begin{array}{l}8 \text { DATE } \\
\text { N/A }\end{array}$ \\
\hline 9 APPROVED CHANGES \\
N/A
\end{tabular}

\section{Element Description}

This element contains bargaining unit personnel, administrative labor and technical personnel from various disciplines. Also included is computer hardware and software, vehicle maintenance, general office supplies and field supplies, and "Termination for Convenience" costs. This element consists of MW treatment activities as follows:

- Tributyl phosphate treatment

- Thermal treatment

- Direct disposal (backlog soils)

- MW characterization

- Smallunique wastes

- Macroencapsulation 


\begin{tabular}{|c|c|c|}
\hline $\begin{array}{l}1 \text { PROJECT TITLE/PARTICIPANT } \\
\text { Solid Waste Program }\end{array}$ & $\begin{array}{l}2 \text { DATE } \\
08 / 31 / 96\end{array}$ & $\begin{array}{l}3 \text { IDENTIFICATION NO. } \\
3.7 .3 .1\end{array}$ \\
\hline $\begin{array}{l}4 \text { WBS ELEMENT CODE } \\
\text { 1.2.1.1.3 }\end{array}$ & \multicolumn{2}{|c|}{$\begin{array}{l}5 \text { WBS ELEMENT TITLE } \\
\text { Transuranic Storage/Transition }\end{array}$} \\
\hline $\begin{array}{l}6 \text { INDEX LINE NO. } \\
1 \mathrm{~A} 13\end{array}$ & $\begin{array}{l}7 \text { REVISION NO. AND } \\
\text { AUTHORIZATION } \\
\text { N/A } \\
\end{array}$ & $\begin{array}{l}8 \text { DATE } \\
\text { N/A }\end{array}$ \\
\hline \multicolumn{3}{|l|}{$\begin{array}{l}9 \text { APPROVED CHANGES } \\
\text { N/A }\end{array}$} \\
\hline $\begin{array}{l}10 \text { SYSTEM DESIGN DESCRIPTION } \\
\text { N/A }\end{array}$ & $\begin{array}{l}11 \text { BUDGE } \\
\text { EW313002 }\end{array}$ & $\begin{array}{l}\text { IING NUMBER } \\
\text { 39EW31302 }\end{array}$ \\
\hline
\end{tabular}

\section{Element Description}

This element contains bargaining unit personnel, administrative labor and technical personnel from various disciplines, as listed in more detail in the attachment. Also included is computer maintenance, vehicie maintenance, general office supplies and field supplies.

Transuranic (TRU) storage is the overall operation and maintenance of the Transuranic Storage and Assay Facility (TRUSAF). TRUSAF provides Resource Conservation and Recovery Act (RCRA) compliant storage of TRU mixed wastes and certified TRU wastes. Newly generated Contact-Handled $(\mathrm{CH})$ TRU waste drums are received at TRUSAF for $x$-ray examinations and TRU content assay. The TRUSAF (224-T Building) processes up to 3,500 $\mathrm{ft3}$ of $\mathrm{CH}$-TRU per year and provides the final quality assurance oversight necessary to assure that the waste complies with the Waste Isolation Pilot Plants (WIPP) Waste Acceptance Criteria (WAC). The assay system is capable of distinguishing whether the TRU content is above or below the $100 \mathrm{nCi} / \mathrm{g}$ limit for Low Level Waste (LLW), thereby reducing the number of drums requiring interim storage. TRUSAF also provides mixed waste storage 
in accordance with WAC 173-303. If drums of waste are below the TRU limit they are sent to the LLW Burial Grounds for disposal or Central Waste Complex for further interim storage.

Funding to perform actual receipt activities is funded by the chargeback/assessment program ML5.

These support functions include engineering analysis, building surveillance and monitoring, facility maintenance and equipment calibration, personnel training and certification, database management, quality assurance and quality control, facility utility costs, design and procurement of support equipment, Treatment, Storage, and Disposal (TSD) statutory/regulatory compliance, facility upgrades, engineering studies through development of functional design criteria, waste certification activities, waste acceptance criteria development, TSD spill prevention, RCRA dangerous waste permit application activities, safety documentation activities, National Environmental Protection Agency documentation activities, operations support, work control, facility cognizant engineering, operations, lab support, technical support, RCRA closure plan activities, sampling activities, health physics, and safety and environmental engineering support activities. This includes activities necessary for the planned transition of the 224-T Facility to EM-40. Activities include development of the memorandum of understanding (MOU) with EM-40, permit closure negotiations, work plan/procedure development, transition training, removal of waste inventory, cell entry preparation, cell characterization, and facility characterization. All inventory is planned for removal by the end of FY97 with transition to occur mid-FY98. 


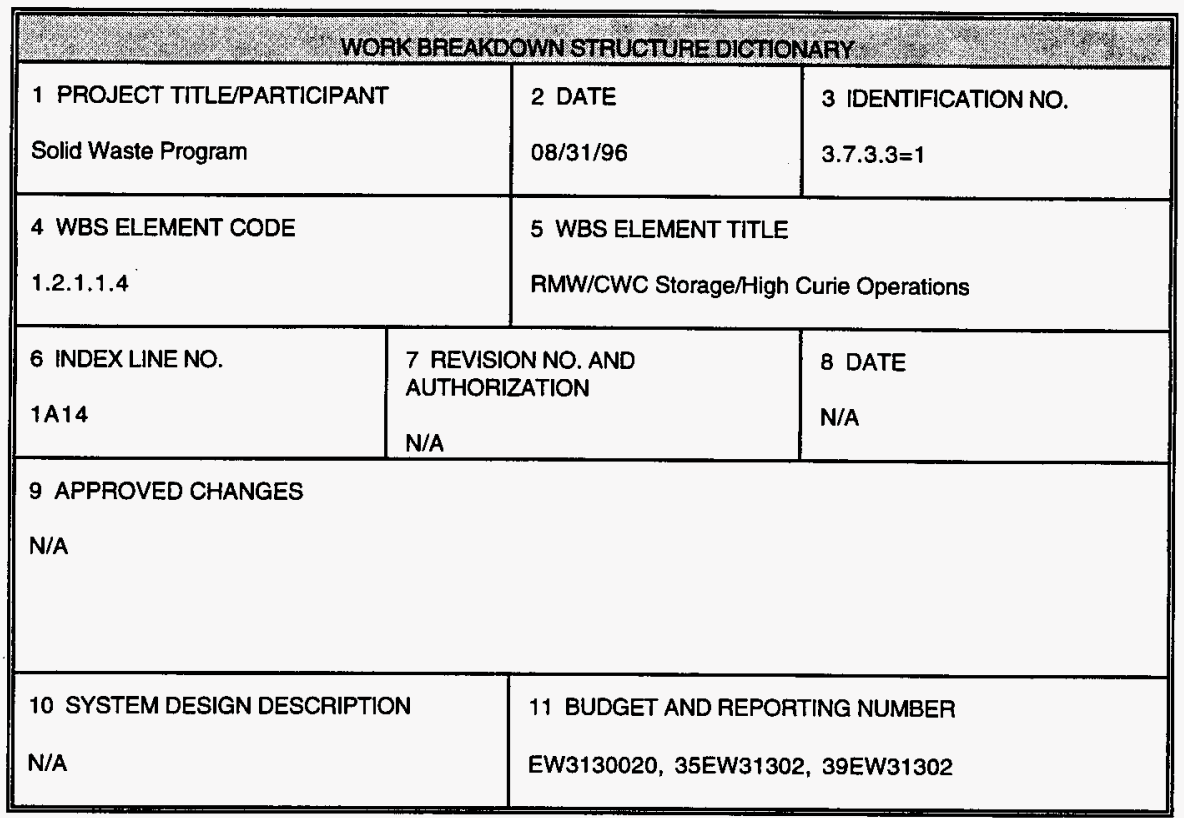

\section{Element Description}

This element contains bargaining unit personnel, administrative labor and technical personnel from various disciplines, as listed in more detail in the attachment. Also included is computer hardware and software, vehicle maintenance, general office supplies and field supplies.

Radioactive Mixed Waste (RMW)/Central Waste Complex (CWC) storage is the overall operation and maintenance of the CWC which provides permitted Resource Conservation and Recovery Act (RCRA) and Toxic Substance and Control Act (TSCA) compliant mixed waste storage for the Hanford site. Mixed waste includes radioactive low level waste and Transuranic (TRU) mixed waste. The CWC also stores other wastes such as contaminated oils, chelating agents and certain other TRU and greater than Class 3 Wastes. Approximately 12,000 cubic feet of mixed waste is received annually. The CWC consists of a series of metal above ground RMW buildings (19 total), the mixed waste storage pad, polychlorinated biphenyls storage facility, low flash point storage modules (12 total) and alkali metal waste 
storage (12 total). These support functions include engineering analysis, building surveillance and monitoring, facility maintenance and equipment calibration, personnel training and certification, data base management, quality assurance and quality control, facility utility costs, design and procurement of support equipment, Treatment, Storage, and Disposal (TSD) statutory/regulatory compliance, facility upgrades, engineering studies through development of functional design criteria, waste certification activities, waste acceptance criteria development, TSD spill prevention, RCRA dangerous waste permit application activities, safety documentation activities, National Environmental Protection Agency documentation activities, operations support, work control, facility cognizant engineering, operations, lab support, technical support, RCRA closure plan activities, sampling activities, health physics, and safety/environmental engineering support activities.

Additional activities provide for acceptance and placement in CWC of the TRU inventory from 224-T and the operations impact from the curie loading associated with this waste inventory. 


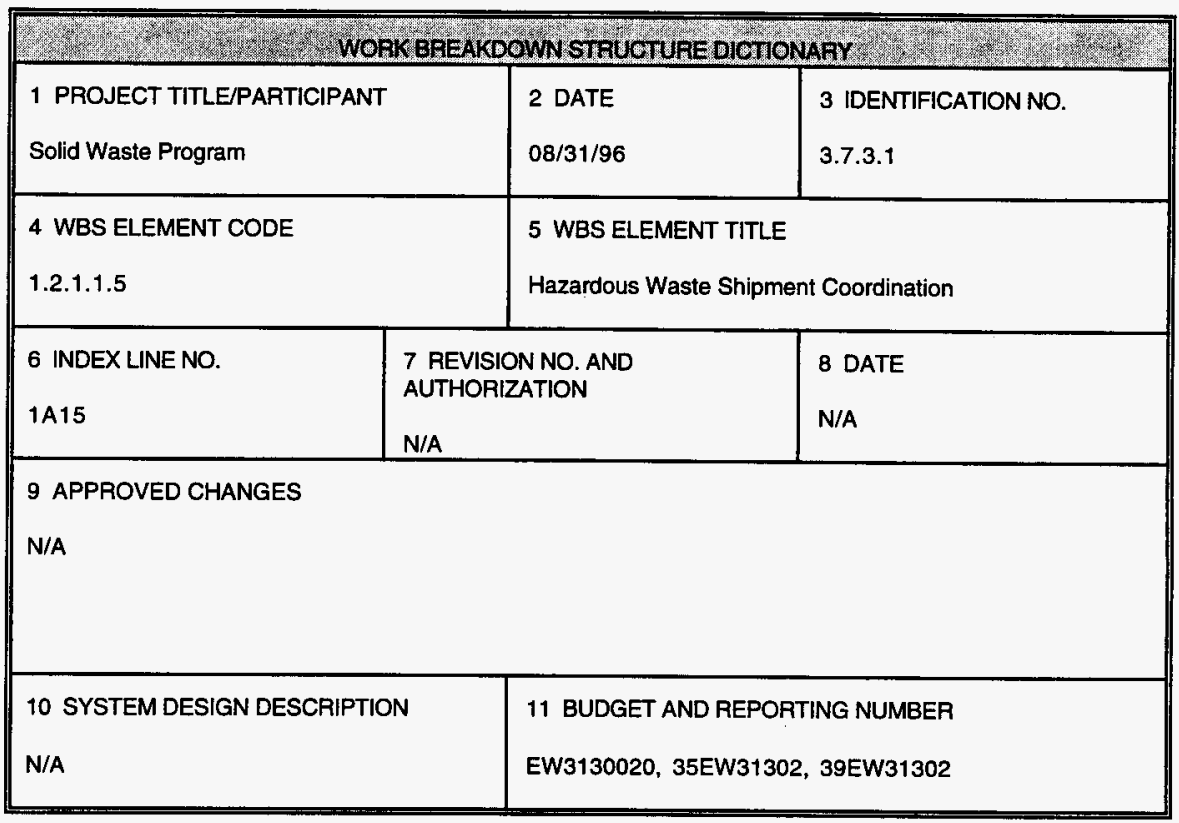

\section{Element Description}

This element contains bargaining unit personnel, administrative labor and technical personnel from various disciplines, as listed in more detail in the attachment. Also included is computer hardware and software, vehicle maintenance, general office supplies and field supplies.

Hazardous Waste Storage Operations is the overall operation and maintenance of the 616 Non-radioactive Dangerous Waste Storage Facility (NRDWSF). The 616 Building serves as a standby storage facility in support of non-radioactive dangerous waste shipments offsite for treatment and disposal. This building provides capability for Toxic Substances Control Act (TSCA) and Resource Conservation and Recovery Act (RCRA) compliant storage of Nonradioactive Dangerous Waste per WAC 173-303. Funding to perform actual shipment activities is funded by the chargeback/assessment program ML8. 


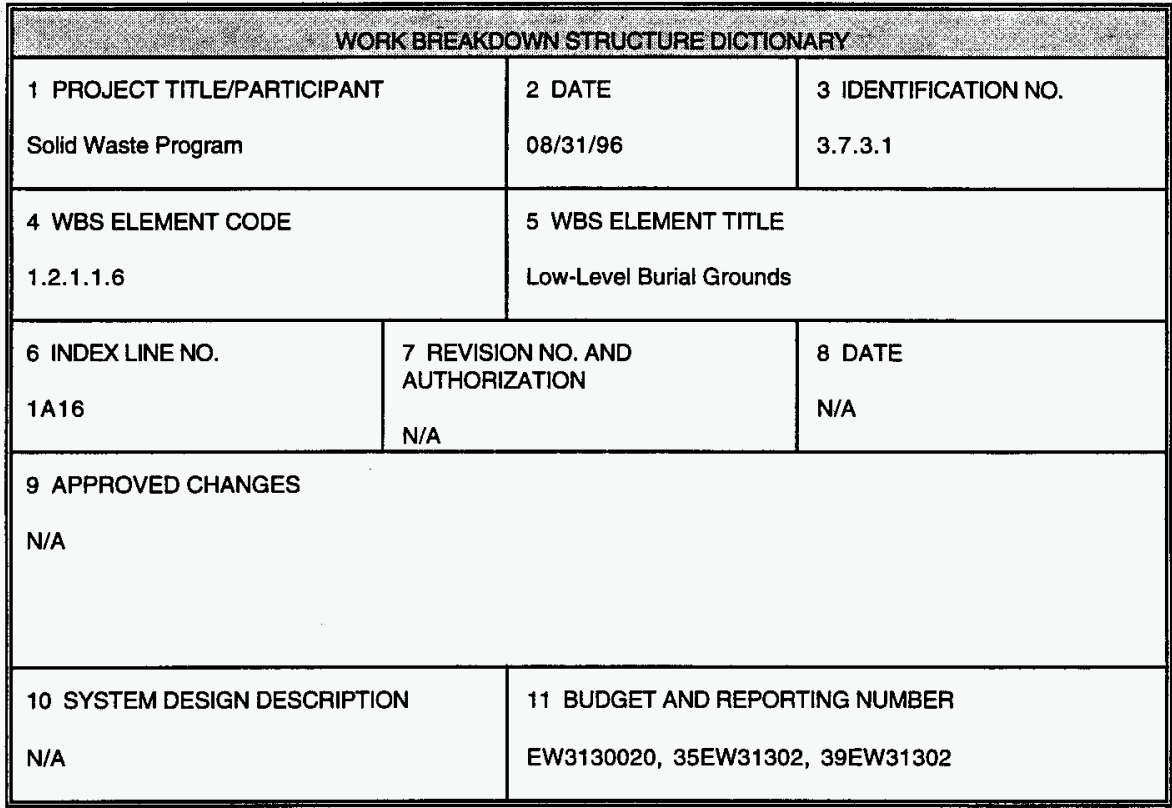

\section{Element Description}

This element contains bargaining unit personnel, administrative labor and technical personnel from various disciplines, as listed in more detail in the attachment. Also included is computer hardware and software, vehicle maintenance, general office supplies and field supplies.

Low Level Waste Burial Ground (LLBG) Operations is the overall operation and maintenance of Hanford's Low Level Waste (LLW) near-surface disposal trenches located in 200 East and 200 West Areas. The main purpose of the LLBG operations is to receive and dispose of LLW from onsite and offsite generators. It receives for disposal approximately 200,000 cubic feet of contact handled and remote-handled waste on an annual basis. The burial grounds are composed of active (unfilled) and new trenches. Active burial grounds include 218-W 3A, 218-W-3AE, 218-W-4C, 218-E-10 and 218-E-12B, (sub reactor compartments), and 218-W$4 B$ (caissons). Future low level burial grounds will include 218-W-6. 
This activity funds managerial, operational, technical, and administrative support to set up and operate the LLBGs. Support functions include engineering analysis, facility surveillance and monitoring, facility maintenance and equipment calibration, personnel training and certification, database management, quality assurance and quality control, facility utility costs, design and procurement of support equipment, Treatment, Storage, Disposal (TSD) Facility statutory/regulatory compliance, TSD spill prevention, Resource Conservation and Recovery Act (RCRA) dangerous waste permit application activities, safety documentation activities (except MSAR), National Environmental Policy Act (NEPA) documentation activities, operations support, work control, facility cognizant engineering, operations, lab support, technical support, sampling activities, health physics, Performance Assessment (PA), and safety and environmental engineering support activities. 


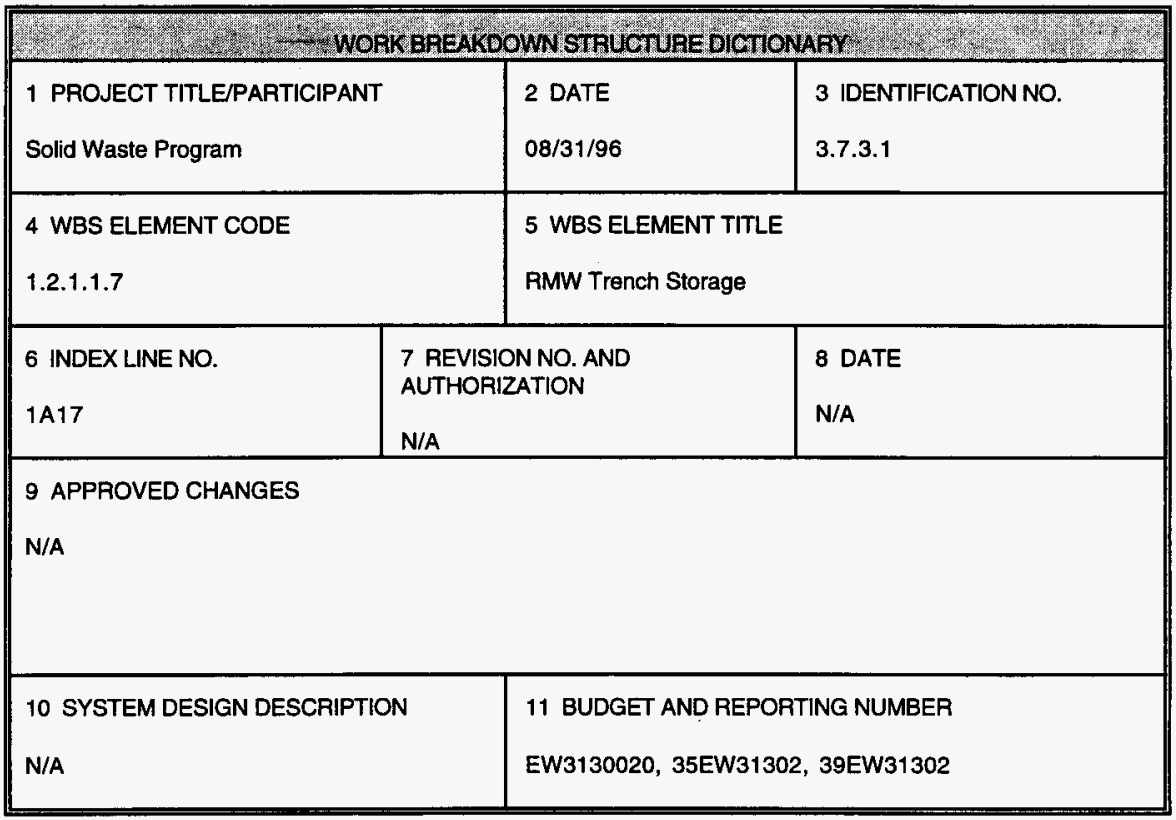

Element Description

This element contains bargaining unit personnel, administrative labor and technical personnel from various disciplines, as listed in more detail in the attachment. Also included is computer hardware and software, vehicle maintenance, general office supplies and field supplies.

Radioactive Mixed Waste (RMW) trench storage is the overall operation and maintenance of the two RMW trenches provided by Project $W-025$. These two trenches are RCRA compliant and landfills which contain double lined leachate collections systems and are located in 218W-5 Burial Ground. They provide disposal capacity for 270,000 cubic feet of low level RMW per trench. The landfill design will allow for operation of heavy equipment and placement of heavy waste loads. The waste is expected to come mainly from onsite waste generators, particularly the Environmental Restoration Program. 
This activity funds managerial, operational, technical, and administrative support to initiate storage operations in the RMW Disposal Trenches. Additionally, it provides funding for engineering and design support for final closure. Support functions include engineering analysis, facility surveillance and monitoring, facility maintenance and equipment calibration, personnel training and certification, database management, quality assurance and quality control, facility utility costs, design and procurement of support equipment, Treatment, Storage and Disposal (TSD) statutory/regulatory compliance, Resource Conservation and Recovery Act dangerous waste permit application activities, safety documentation activities, operations support, work control, facility cognizant engineering, operations, lab support, technical support, RCRA closure plan activities, sampling activities, health physics, and safety and environmental engineering support activities. 


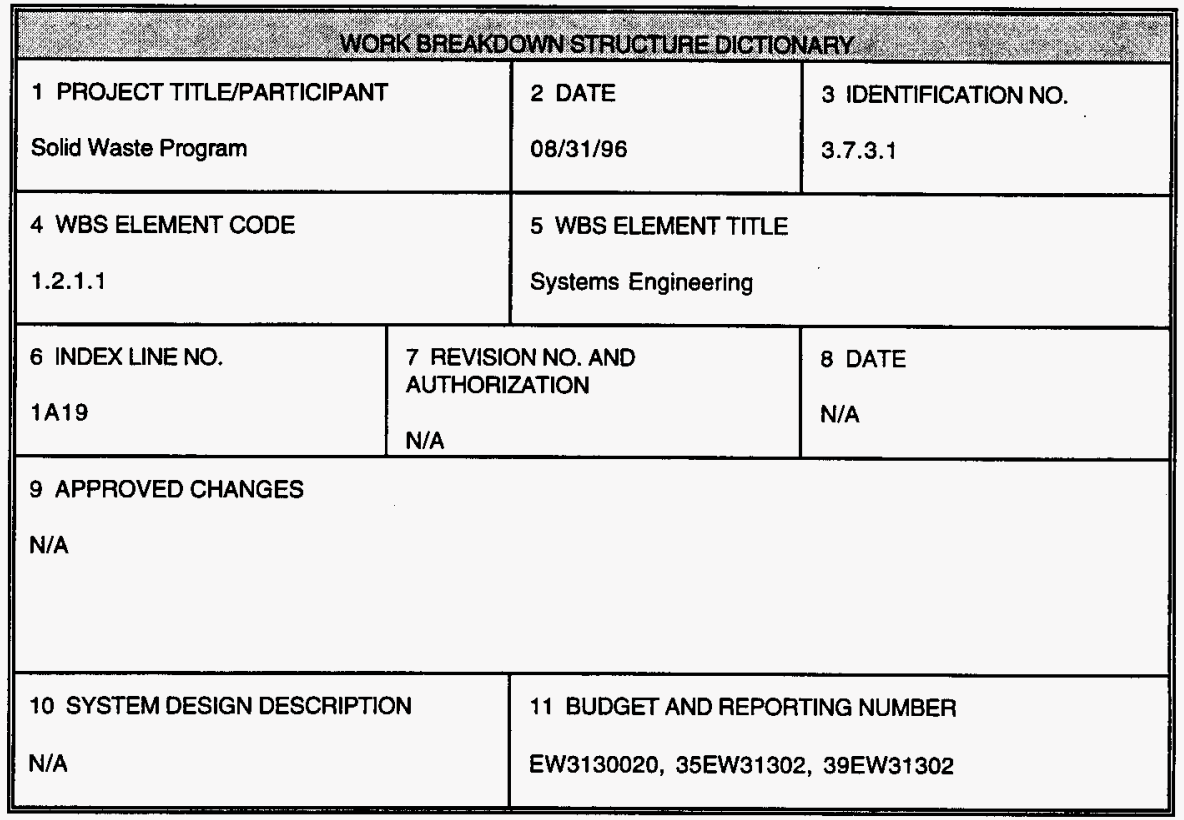

\section{Element Description}

This element contains administrative labor and technical personnel from various disciplines. Also included are computer hardware and software, vehicle maintenance and general office supplies. This element consists of Systems Engineering activities as follows:

- Solid Waste Technical Baseline Document

- Interface Control Documents

- Systems Analysis

- Risk Assessment

- $\quad$ Site-Wide Systems Engineering

- Environmental Management System

- Site Integrated Schedule

- DNFSB 94-2 


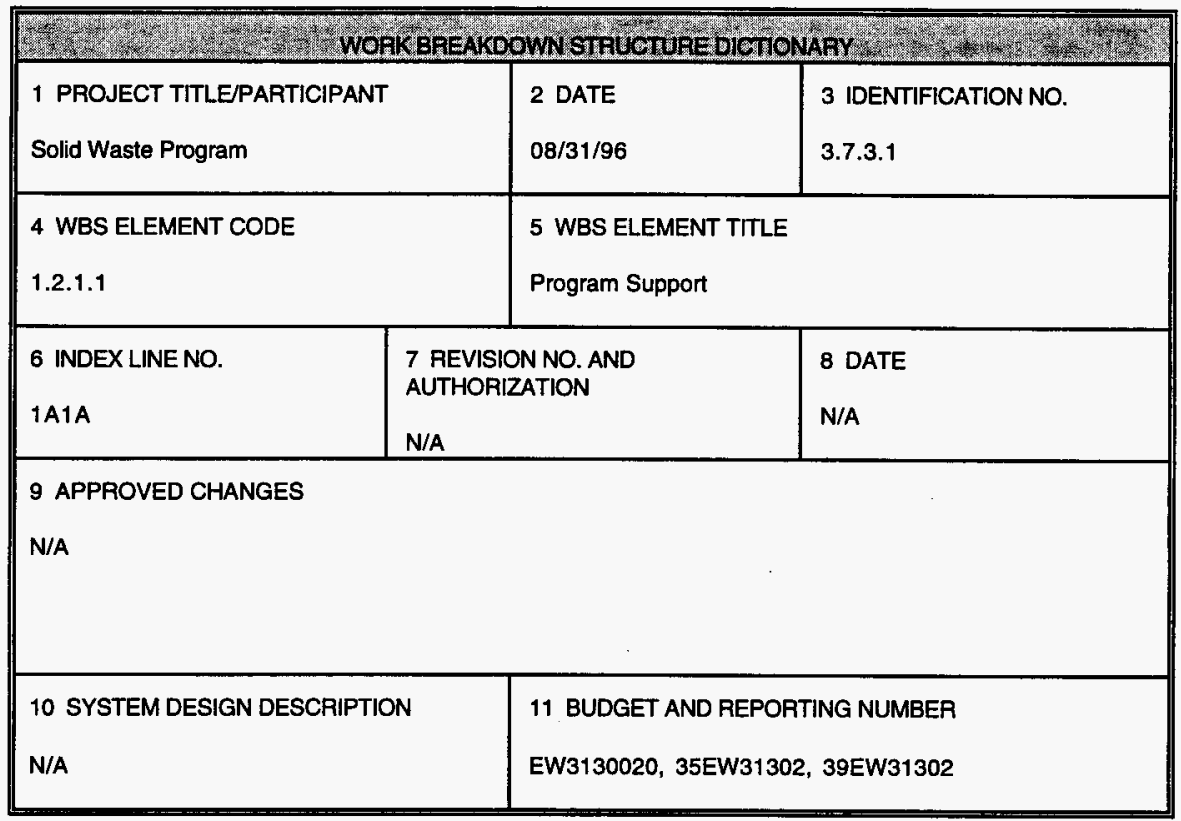

\section{Element Description}

This element contains administrative labor and technical personnel from various disciplines. Also included are computer hardware and software, and general office supplies. This element consists of Program Support activities as follows:

- Program Management (1A1A01)

- DOE Walk-in Work (1A1A06)

- Data Management (1A1A07)

- Safety and Health Integration (1A1A08)

- Master SAR (1A1A04)

- SW EIS (1A1A05) 


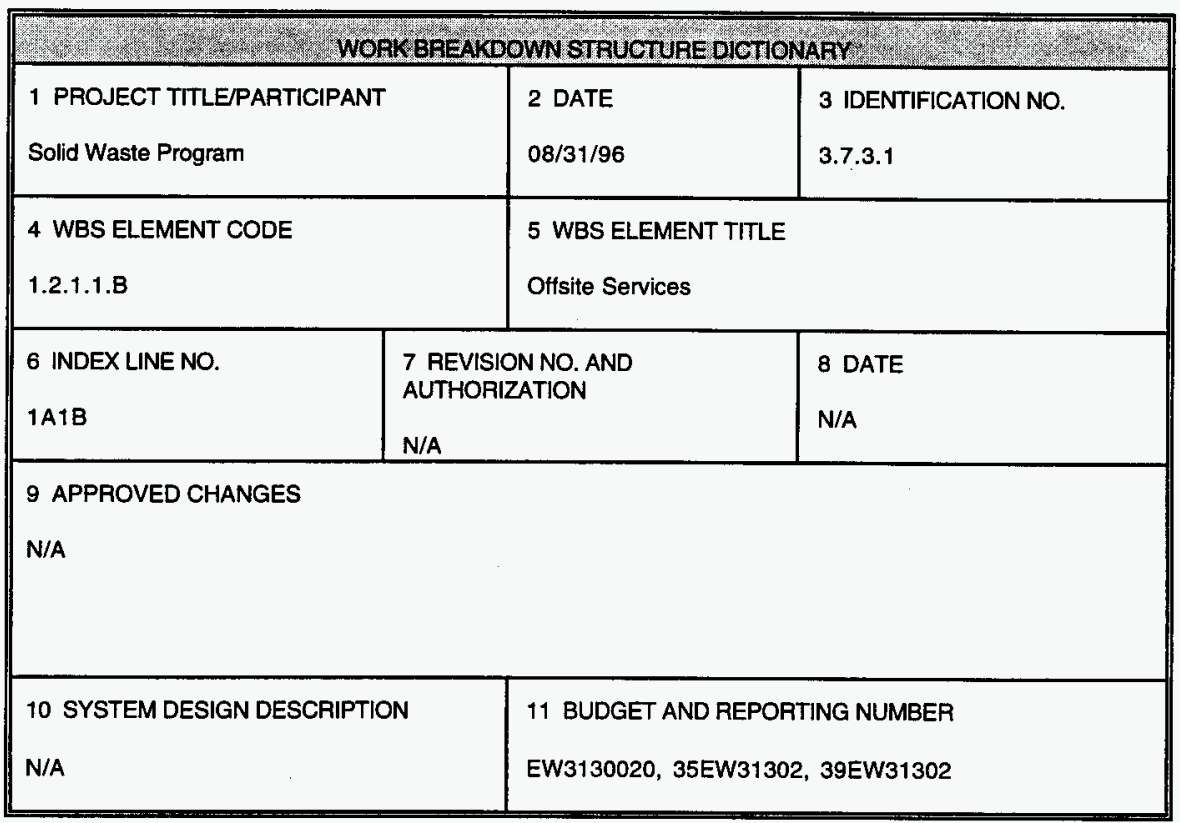

\section{Element Description}

This element contains administrative labor and technical personnel from various disciplines, as listed in more detail in the attachment. Also included are computer hardware and software, and general office supplies.

This product line represents work for off-site contractors (other U.S. Department of Energy offices and specialized agencies, i.e., PSNS) provided by Solid Waste Management. Three key areas are encompassed by this product line activity. These areas are waste receipt and handling of offsite low-level and low-level mixed wastes, off-site generator assistance, acceptance of the Navy's Defueled Submarine Reactor Compartments (SRC), and Federal Facilities Compliance Act (FFCA) technical support to the U.S. Department of Energy Richland Office (RL). Funding is provided via DOE interworks, MPO, funding (fin plan), or other approved funding mechanism, and is performed on an as requested/needed basis. 
Offsite Generator Assistance is provided via the Generator Assistance Program (GAP). This program is responsible for direct point of contact support to all offsite generators shipping low-level and radioactive mixed waste to the Hanford Site CWC and Burial Grounds. The GAP assists generators in all developmental phases of waste certification including program planning, Quality Assurance, working level procedures, training, and complete waste characterization and designation. Further support to offsite generators is provided to reclassify and maintain their program status from a limited to full approval status.

This product line further provides for the acceptance and disposal of the Navy's defueled Submarine Reactor Compartments (SRC) and other ancillary DOE wastes such as KAPL pumps, KAPL core baskets, Bettis pumps, and other Bettis wastes. Activities include preparation of waste acceptance criteria and promulgation of this criteria to the Navy via approved documentation, providing all estimates and schedules, and obtaining funding from DOE or the Navy in support of SRC disposition. Operations and maintenance of the Navy Trench (218-E-12B, Trench 94) is provided under this activity through trench preparation and construction of SRC foundations in accordance with the Navy design and applicable Hanford procedures.

Prior to receipt of waste, all necessary permits and completed documentation (e.g., Acceptance and Transport Movement Checklist) are prepared and approved. Inspection and acceptance of waste packages as they are received to verify they meet the waste acceptance criteria is also performed. For SRC disposal a Transport Manager to represent $R L$ and the Navy during waste movements is provided under this activity to coordinate onsite organizations and offsite contractors for the safe and efficient off-loading and transport of these large waste packages for disposal at their final destination. 


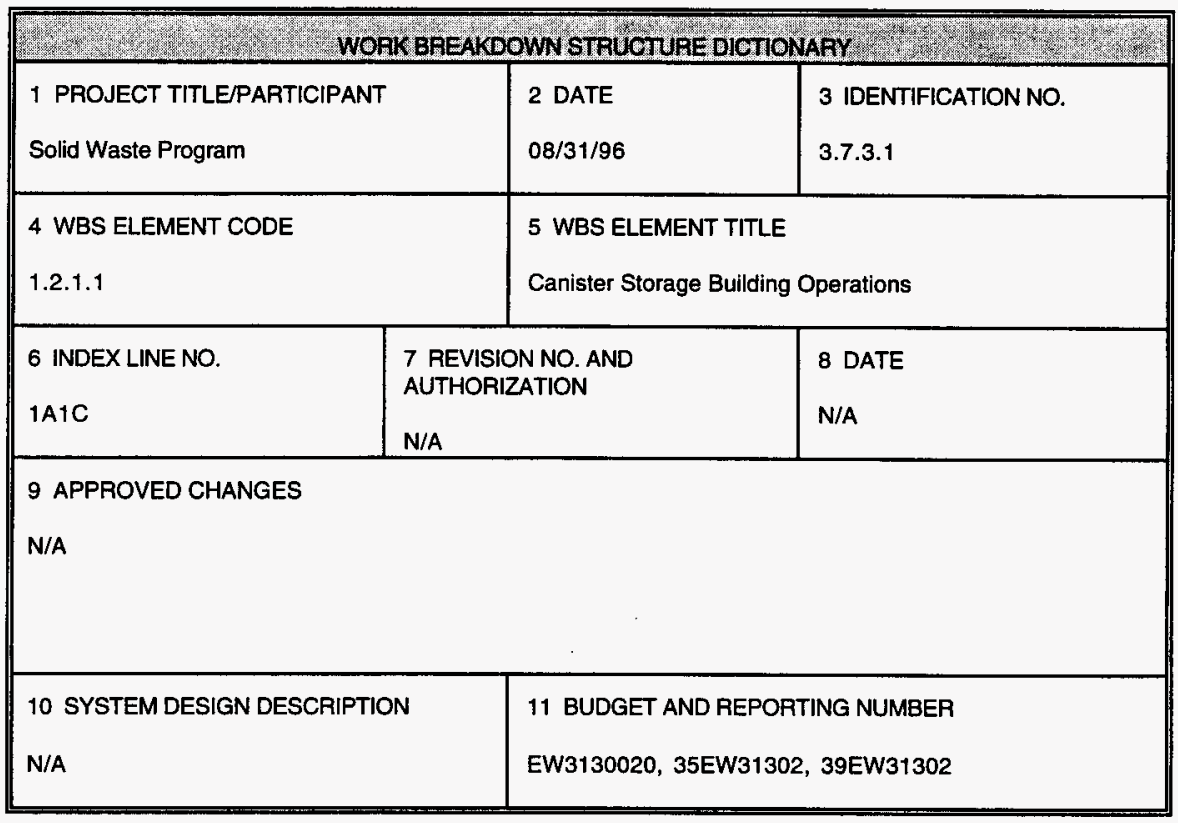

\section{Element Description}

This element contains administrative labor and technical personnel and bargaining unit personnel from various disciplines. This activity is for the operation and maintenance, beginning in FY2001, of the 212-H Canister Storage Building (CSB) after the design, construction, and placement of fuel in the CSB by SNF and the hot conditioning process of the spent nuclear fuel. The new structure is designed to safely store about 2100 metric tons of irradiated metallic uranium fuel for about $\mathbf{4 0}$ years, or until the fuel is sent to a repository or otherwise dispositioned. This fuel is currently stored in the K Basins, but the basins have exceeded their design lives, and the fuel is deteriorating due to corrosion. The CSB is a reinforced concrete vault structure with about 220 tubes designed to hold containers (referred to as Multi-Canister Overpacks - MCOs) of K Basin spent nuclear fuel, with the tubes providing secondary containment of the radioactive fuel at Hanford. 


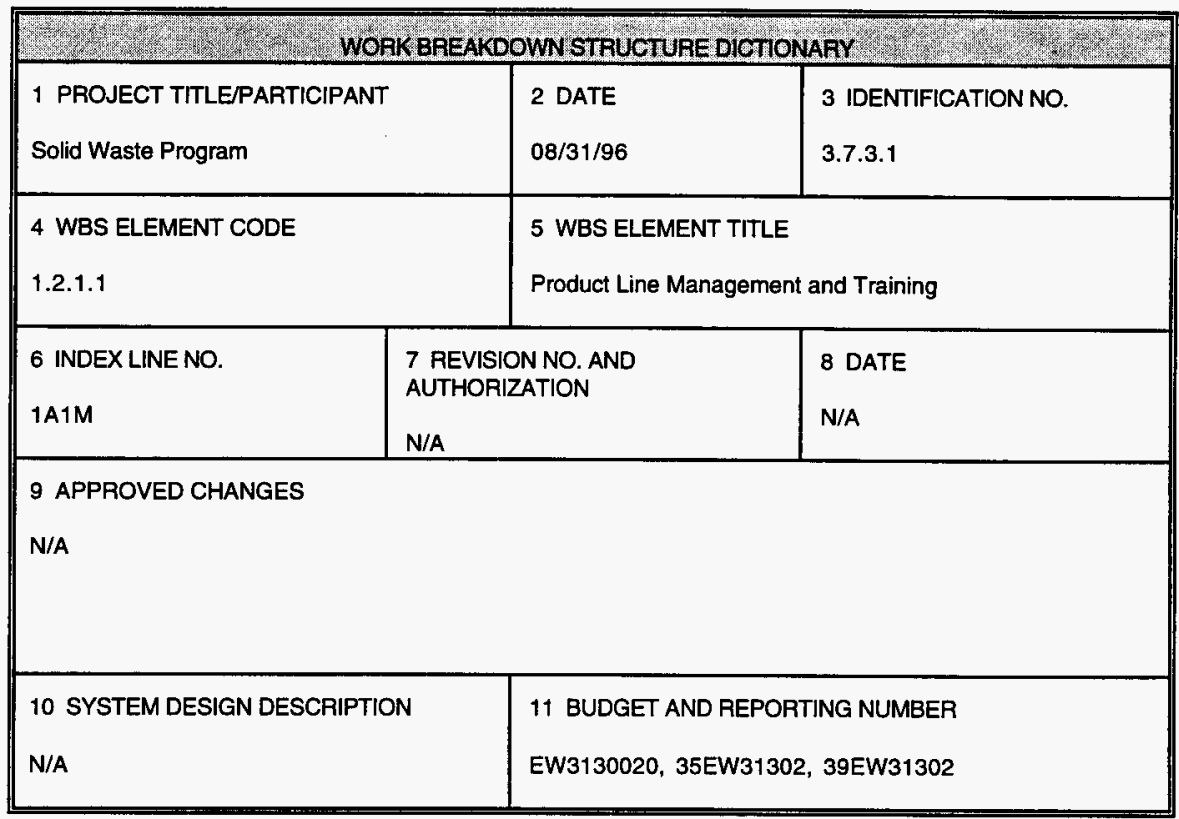

\section{Element Description}

This element contains administrative labor and technical personnel and bargaining unit personnel from various disciplines. Also included is tuition costs for training, contract costs for records management activities, and costs associated with the patrol allocation. This element consists of Management/Training activities as follows:

- Management activities

- Training activities

- Records Management

- Budget/schedule activities

- QA activities

- Patrol Allocation 


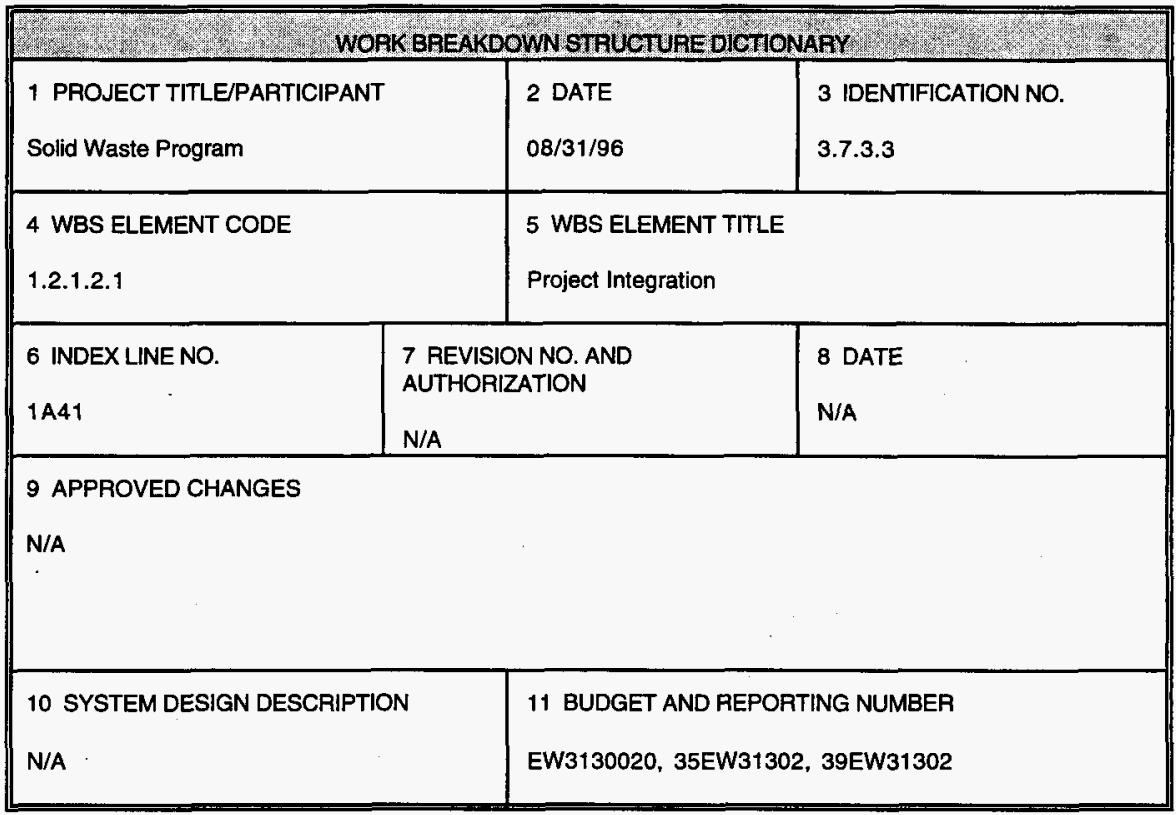

\section{Element Description}

This element contains administrative labor and technical personnel from various disciplines. Also included is computer training, hardware and software, and general office supplies. This activity provides project management for financial support and scheduling for the Waste Receiving and Processing (WRAP) 1 including the following activities: baseline management, change control administration, Site Management System implementation, project management plans, document control and records management, project engineering support and the development/maintenance of integrated schedules. Interface with Solid Waste Programs, review activity data sheets, and support five year planning. Also provides management of Architect/Engineer, and construction manager. Will provide validation review packages, Energy System Acquisition review support, Independent Cost Estimate support, and the associated reporting to U.S. Department of Energy, Richland Operations Office/U.S. Department of Energy Headquarters. 


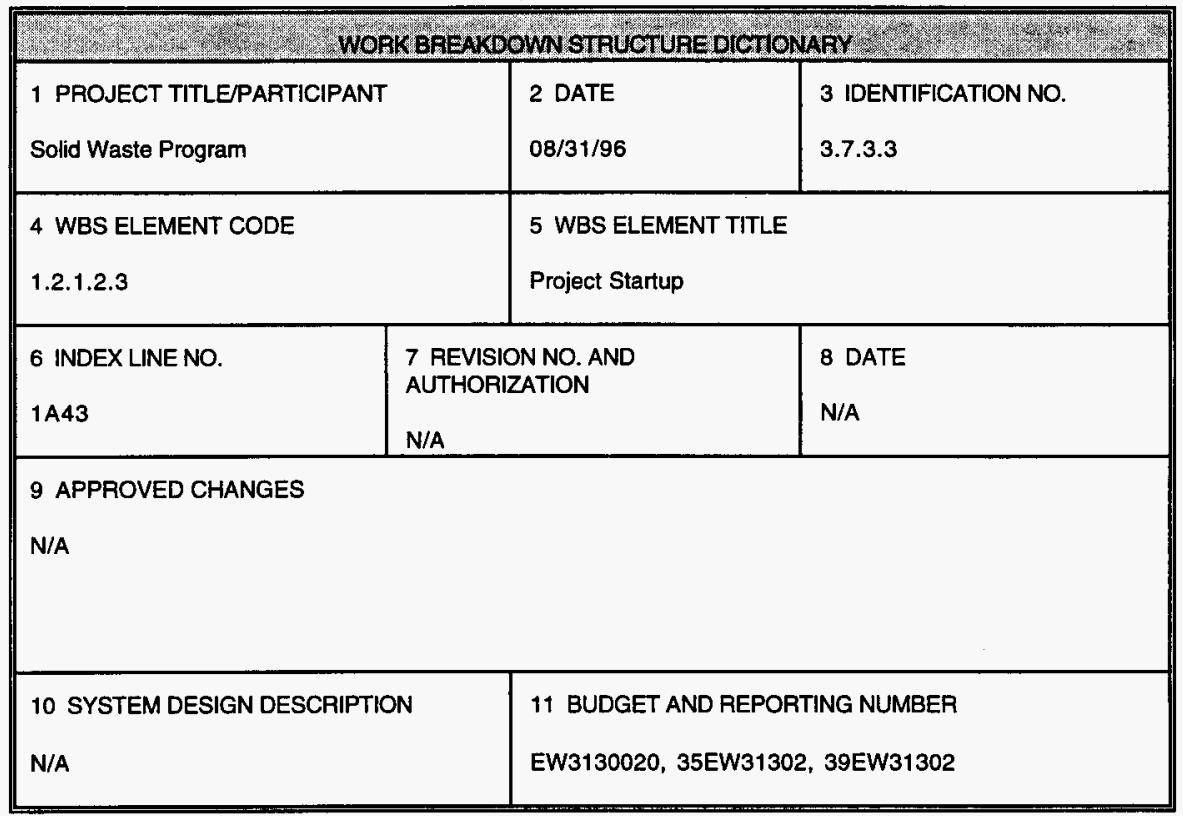

\section{Element Description}

This element contains administrative labor and technical personnel from various disciplines. Also included is travel, material, and equipment required for project startup. This activity provides operational preparedness and startup planning associated with the total project cost for the Waste Receiving and Processing 1. Prepare operational test procedures and operational readiness review documents. Will provide management review and engineering support to review project documents and responding information requests. Provide expertise to review the adequacy of vendor design and concepts from a facility operations feasibility perspective. Support planning and integration efforts including support permitting activities. 


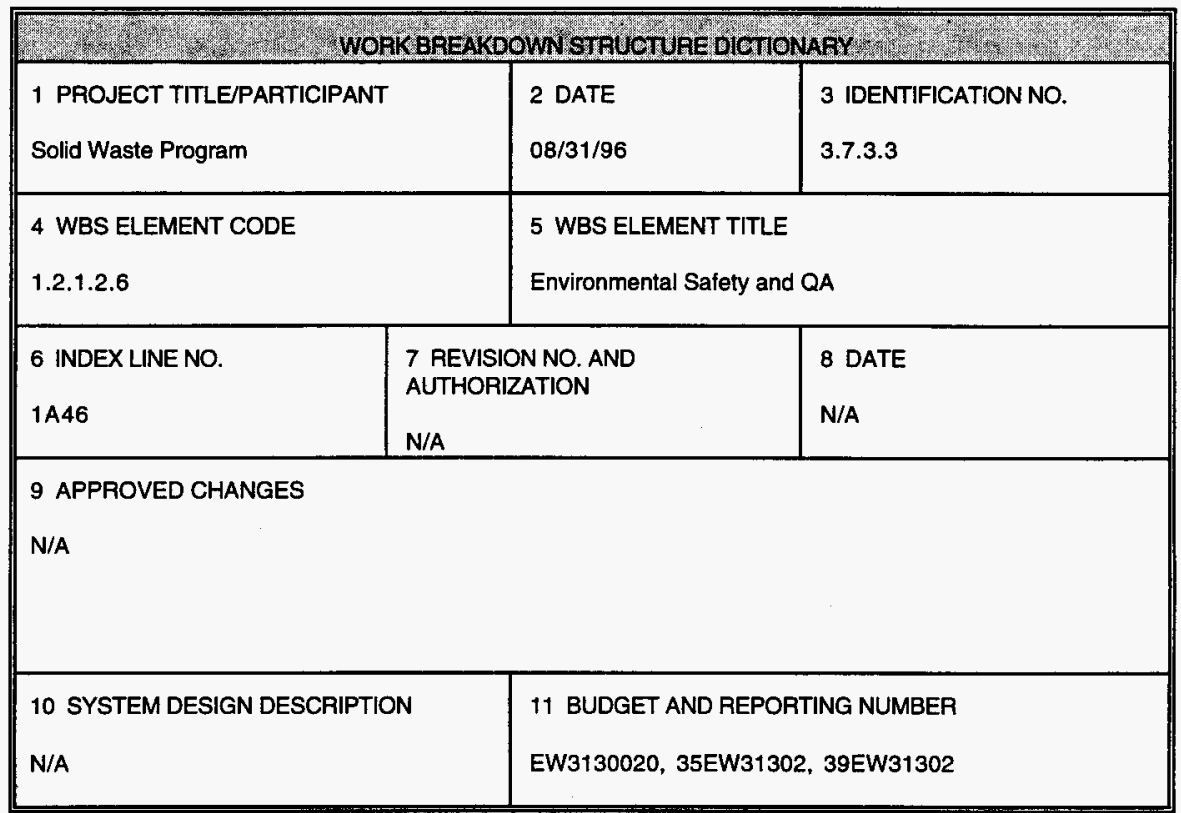

\section{Element Description}

This element contains administrative labor and technical personnel from various disciplines. Also included is PO contracts, travel, training, and general office supplies. This activity provides management review and engineering support to include review of project documents and responding to information requests. Will provide for completion of Final Safety Analysis Report (FSAR) for WRAP. Will provide update to Quality Assurance Project Plan. 


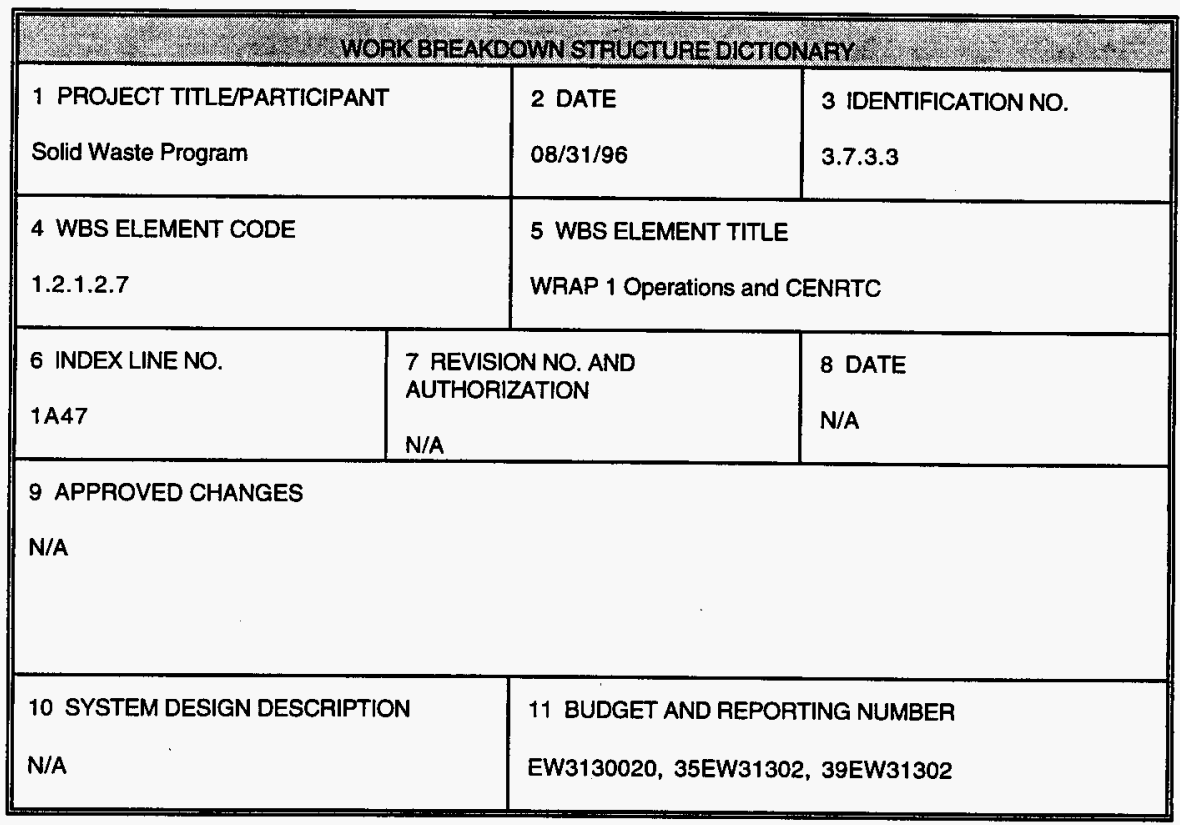

\section{Element Description}

This element contains administrative labor and technical personnel and bargaining unit personnel from various disciplines. Also included is training, travel, network access, relocation, site telephone, computer hardware and software, and general office supplies. This activity provides support for commencing the Waste Receiving and Processing 1 Operations on or before March 31, 1997, to meet Hanford Federal Facility Agreement and Consent Order Milestone $\mathrm{M}-18-00$. The activities include preparation of emergency response plans; development of waste acceptance, sampling, and batching plans; development of operating, maintenance, emergency and administrative procedures; development of waste minimization and handling plans; and technical support for permit and Final Safety Analysis Report development. This includes CENRTC funding for FY1997/1998 to acquire facility spares. This activity also covers coding the DMS software and software testing. 


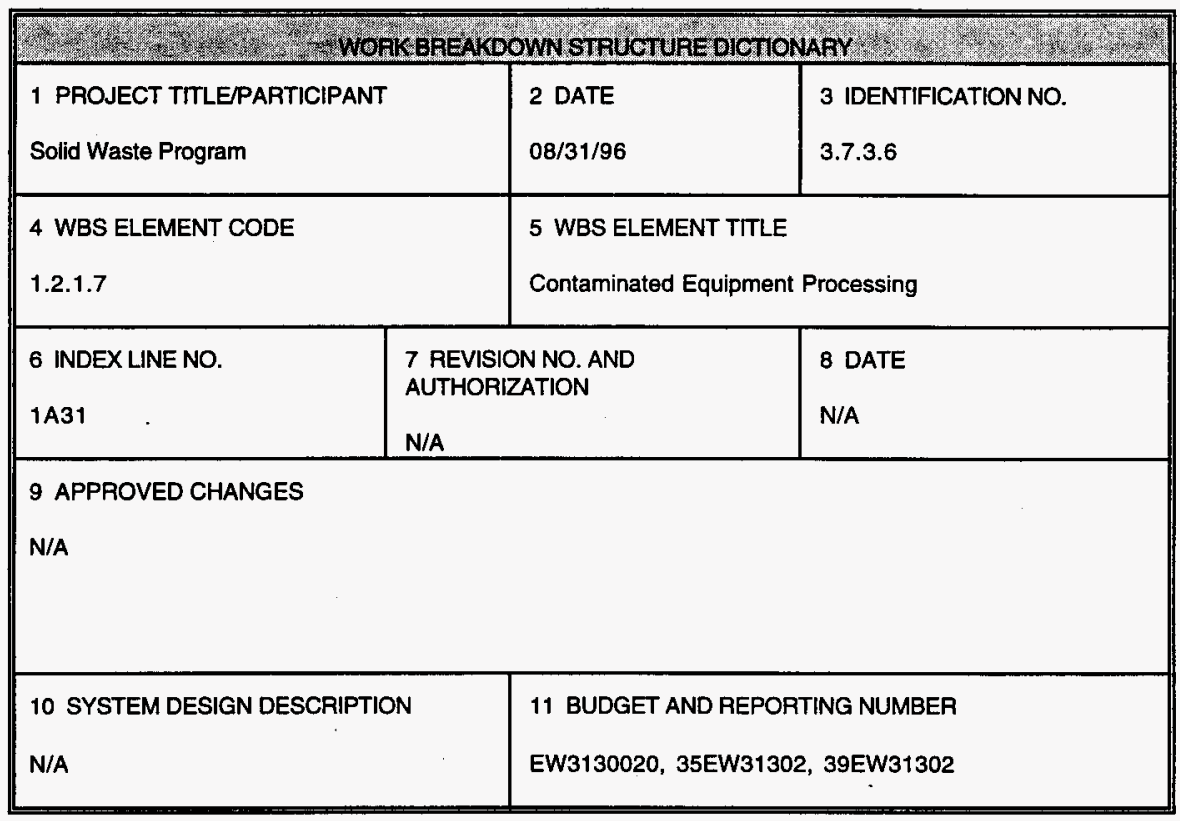

\section{Element Description}

This element contains bargaining unit personnel, administrative labor and technical personnel from various disciplines to support 221-T Facility product processing, maintenance activities, environmental permitting, waste minimization/ALARA, and site non-compliance response activities. Also included are the facility assessments for the year. 
Canyon processing includes processing of high dose rate waste and contaminated equipment in accordance with the Hanford Site Solid Waste Acceptance Criteria, WHC-EP0063-4, for disposal, re-use, or free release. The 221-T canyon processing activities include:

- Provide support for required permits.

- $\quad$ Support plant modifications necessary for product processing in 221-T.

- Identify and purchase equipment required for size reduction and decontamination.

- Provide waste stream forecasting.

- Utilize a variety of decontamination techniques to reduce the volume of the contaminated component of the waste.

- Reduce the size of large pieces of waste and equipment to fit in burial containers.

- Package waste for burial.

- Decontaminate, repair, and return to service reusable failed equipment.

- The equipment to be processed will consist of Tank Farms augers and contaminated equipment stored in the T Plant canyon. In addition, other high dose rate contaminated equipment and waste being stored at site facilities undergoing decommissioning and generated during the site cleanup will be treated. Treatment of high dose rate waste and equipment will take place in the 221-T canyon.

- Perform decontamination on equipment and debris utilizing $\mathrm{CO}_{2}$ decontamination equipment and methods. 


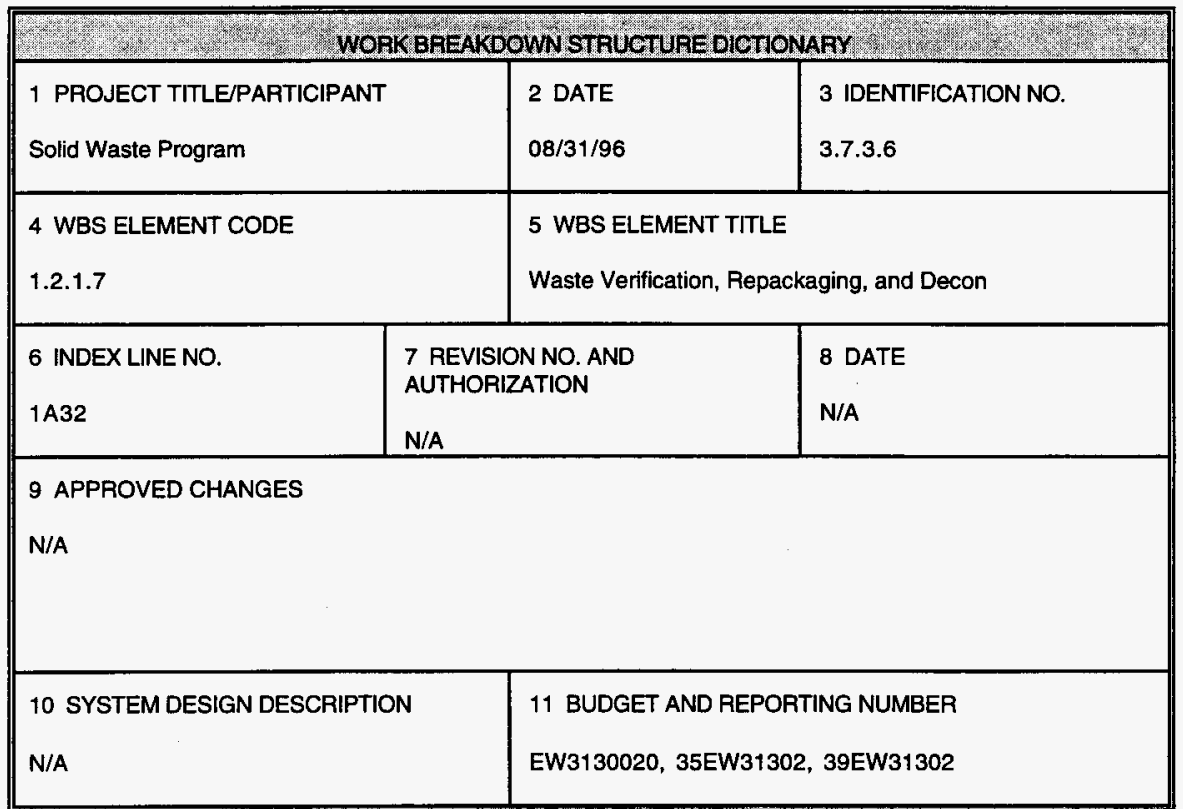

\section{Element Description}

This element contains bargaining unit personnel, administrative labor and technical personnel from various disciplines to support 2706-T Facility by maintaining compliant conditions, product processing, maintenance activities, SAR upgrades and ISB, environmental permitting, waste minimization/ALARA, site non-compliance response activities, upgrade projects and cost account plan management. Also included are the facility assessments for the year. 
Low level processing includes 2706-T hot standby activities and 2706-T product processing activities.

The hot standby work will provide safe, monitored storage for contaminated equipment located in the 2706-T facility. This work reflects the minimum scope needed to ensure public safety and environmental protection until contaminated equipment/ materials are removed from 2706-T. The 2706-T hot standby activities include:

- Provide technical support for DOE Order 5480.21 review.

- Inspection, operation, and maintenance of equipment needed to maintain the 2706-T facility in a safe configuration, including radiation monitoring equipment, fire protection and other safety equipment.

- Operation, surveillance, and maintenance of liquid waste handling equipment and ventilation systems.

- Administration and training associated with maintaining a safe, compliant facility.

- $\quad$ Preparation of T Plant SAR, ECNs, PSARs, FSARs.

The 2706-T product processing work will include processing of low dose rate waste and contaminated equipment in accordance with the Hanford Site Solid Waste Acceptance Criteria, WHC-EP-0063-4, for disposal, re-use, or free release per 10CFR835. The 2706-T product processing activities will include:

- $\quad$ Provide support for required permits.

- Support 2706-T plant modifications necessary for product processing.

- Identify and purchase equipment required for size reduction and decontamination.

- $\quad$ Provide waste stream forecasting.

- Utilize a variety of decontamination techniques to reduce the volume of the contaminated component of the waste. Reduce the size of large pieces of waste and equipment to fit in burial containers.

- Package waste for burial.

- Decontaminate, repair, and return to service reusable failed equipment.

- The equipment to be processed will consist of customers cranes, vehicles, rail cars, trucks, and tools. In addition, other facility services include the sampling, inspection of 
low level waste and radioactive mixed waste/equipment, inventory/verification of constituents and repackaging for disposal. 


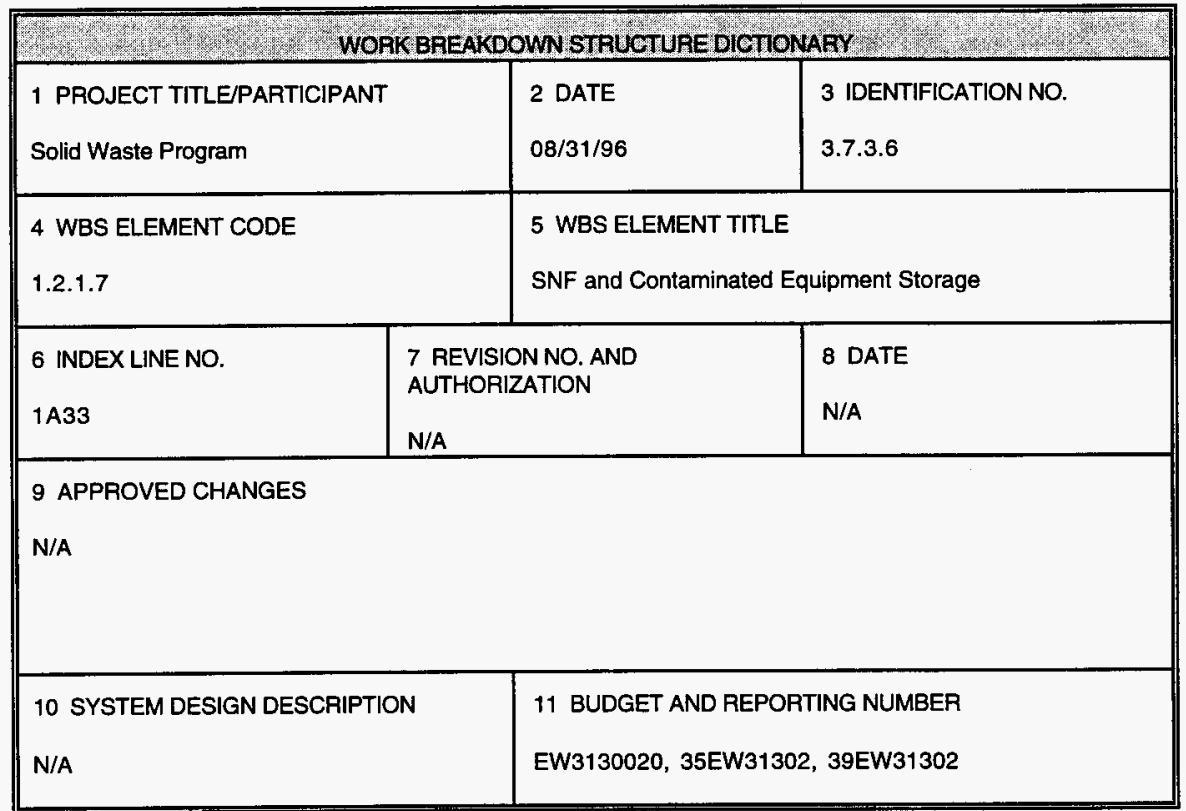

\section{Element Description}

This element contains bargaining unit personnel, administrative labor and technical personnel from various disciplines to support 221-T/271-T Facility cold standby, hot standby, and PWR pool storage and maintenance of PWR pool equipment, by maintaining compliant conditions, maintenance activities, SAR upgrades and ISB, environmental permitting, waste minimization/ALARA, site non compliance response activities, upgrade projects and cost account plan management. Also included are the facility assessments for the year. 
Contaminated equipment storage includes 221-T/271-T cold standby activities, hot standby activities, and PWR pool storage and maintenance of PWR pool equipment.

The 221-T cold standby work and the hot standby work will include providing safe, monitored storage for contaminated equipment located in the 221-T facility. This activity reflects the minimum scope needed to ensure public safety and environmental protection until contaminated equipment and materials are removed from 221-T. This scope includes Project C-157, Electrical Upgrades.

The PWR pool work includes the storage of 72 spent fuel elements, and one non-irradiated element at T Plant in accordance with the latest revision of WHC-SD-CP-SAR-007, "T Plant Safety Analysis Report". Also included is the storage of the pieces of contaminated equipment shown on figure 1 of the "T Plant Canyon Towers Work Plan", SW-PE-94-001 (April 1994) in aocordance with the most recent revision of WHC-SD-CP-SAR-007. This activity reflects the minimum scope needed to ensure public safety and environmental protection until Spent Nuclear Fuels and all contaminated equipment and materials are removed from T Plant.

The contaminated equipment storage activities include:

- Provide technical support for DOE Order reviews.

- Inspection, operation, and maintenance of equipment needed to maintain the 221-T facility and the PWE pool in a safe configuration, including radiation monitoring equipment, fire protection and other safety equipment.

- Operation, surveillance, and maintenance of liquid waste handling equipment and ventilation systems.

- Administration and training associated with maintaining a safe, compliant facility.

- Preparation of T Plant SAR, ECNs, PSARs, FSARs.

- Support plant modifications necessary for the product line.

- Prepare for steam removal in the facility by replacing all systems that are dependant on steam.

- $\quad$ Support 221-T Canyon transition activities. By TPA agreement, liquid-based decontamination must be removed from the T Plant canyon by the end of FY99. Activities in the account include canyon cleanout, spent fuel removal, decontamination operations relocations and future mission planning. 


\begin{tabular}{|c|c|c|}
\hline $\begin{array}{l}1 \text { PROJECT TITLE/PARTICIPANT } \\
\text { Solid Waste Program }\end{array}$ & $\begin{array}{l}2 \text { DATE } \\
08 / 31 / 96\end{array}$ & $\begin{array}{l}3 \text { IDENTIFICATION NO. } \\
3.7 .3 .6\end{array}$ \\
\hline $\begin{array}{l}4 \text { WBS ELEMENT CODE } \\
1.2 .1 .7\end{array}$ & \multicolumn{2}{|c|}{$\begin{array}{l}5 \text { WBS ELEMENT TITLE } \\
\text { LWTC Maintenance and Certification }\end{array}$} \\
\hline $\begin{array}{l}6 \text { INDEX LINE NO. } \\
1 \text { A34 }\end{array}$ & $\begin{array}{l}7 \text { REVISION NO. AND } \\
\text { AUTHORIZATION } \\
\text { N/A }\end{array}$ & $\begin{array}{l}8 \text { DATE } \\
\text { N/A }\end{array}$ \\
\hline \multicolumn{3}{|l|}{$\begin{array}{l}9 \text { APPROVED CHANGES } \\
\text { N/A }\end{array}$} \\
\hline $\begin{array}{l}10 \text { SYSTEM DESIGN DESCRIPTION } \\
\text { N/A }\end{array}$ & \multicolumn{2}{|c|}{$\begin{array}{l}11 \text { BUDGET AND REPORTING NUMBER } \\
\text { EW3130020, 35EW31302, 39EW31302 }\end{array}$} \\
\hline
\end{tabular}

\section{Element Description}

This element contains bargaining unit personnel, administrative labor and technical personnel from various disciplines to support railcar maintenance/certification by maintaining compliant conditions, maintenance activities, environmental permitting, waste minimization/ALARA, and site non compliance response activities. 
This activity assures the availability of at least one liquid waste tank car (LWTC) for the transport of liquid waste, generally from T Plant, the 300 area labs, and the 400 area to Tank Farms. The LWTCs also serve as site backup liquid waste transfer for Hanford site facilities currently utilizing hard piping. LWTC services are essential to maintain $<90$ day storage requirements for facilities that do not have liquid waste storage permits, and for storage tank liquid level control. Railcar activities include:

- The performance of required corrective and preventive maintenance on the LWTCs.

- Certification of the LWTCs on a regular basis to assure they meet safety requirements as specified in T Plant's Safety Analysis Report (SAR) for continued service.

- The LWTCs now in use (HO-18581, -18582) will be retired as their replacements (HO10T-3663, -35664) are placed in service. 


\begin{tabular}{||l|l|l||}
\hline \multicolumn{3}{|c|}{ WORK BREAKOOWN STRUGTURE DICTIONARY } \\
\hline $\begin{array}{l}\text { Solid Waste Program } \\
\text { PROJECT TITLE/PARTICIPANT }\end{array}$ & $\begin{array}{l}\text { DATE } \\
08 / 31 / 96\end{array}$ & $\begin{array}{l}3 \text { IDENTIFICATION NO. } \\
3.7 .3 .2\end{array}$ \\
\hline $\begin{array}{l}\text { 1.2.1.9.1 WBS ELEMENT CODE } \\
\text { 6 INDEX LINE NO. }\end{array}$ & $\begin{array}{l}5 \text { WBS ELEMENT TITLE } \\
\text { T Plant Secondary Containment/Leak Detection Upgrades, } \\
\text { Project W-259 }\end{array}$ \\
AB1 & $\begin{array}{l}7 \text { REVISION NO. AND } \\
\text { AUTHORIZATION } \\
\text { N/A }\end{array}$ & $\begin{array}{l}\text { 8 DATE } \\
\text { N/A }\end{array}$ \\
\hline $\begin{array}{l}\text { 9 APPROVED CHANGES } \\
\text { N/A }\end{array}$ & 11 BUDGET AND REPORTING NUMBER \\
\hline EW3130020, 35EW31302, 39EW31302 \\
\hline N/A SYSTEM DESIGN DESCRIPTION
\end{tabular}

\section{Element Description}

Provides funding for project design, preparation of engineering documentation, procurement, purchase, and installation of secondary containment and leak detection upgrades.

This line item will provide compliant tank and piping systems for ongoing T Plant maintenance and decontamination operations and meet Hanford Federal Facility Agreement and Consent Order milestone M-32-03 due in FY 1999.

Project W-259 will provide compliant liquid waste collection, storage, and transfer systems for the 2706-T and 2706-TA facilities. 


\section{W-259 Project Integration}

- Administration-staff \& safety meetings, HGET, and Site general training.

- Routine Performance Reports/Schedules-EAC, VAR, Mid-year, Functional Review Schedule Updates, and associated meetings.

- Project Support-manager, secretary, CAM, financial \& scheduling, support on upkeep activities for project: contract negotiations, ECN review, change control, and CAP/schedule maintenance.

Document Control \& Records Management

- Project Plans \& Validation-manager, CAM, financial \& scheduling, support on: PMP, CPDS, SDRD, validation, ICE review.

- $\quad$ ADS/MYPP Development-manager, CAM, financial \& scheduling, support.

\section{W-259 Environmental, Safety \& QA}

- Environmental Oversight-permitting plan, Environmental Permit Application, project document oversight, project close-out \& record review.

- Safety Oversight-PSE review, SAR/ISB analysis, SAR revision, project document oversight, project close-out \& record review.

- Quality Assurance Oversight-QA Program Plan, project document oversight, project close-out \& record review.

- W-259 Design/Construction Support

- Definitive Design Support

- Title III Support

- Construction Support

- $\quad$ ATP Development \& Performance Support

- W-259 Project Startup

- OTP Development \& Performance

- $\quad$ Procedures Development \& Update 
- $\quad$ As-built Drawings Support

- Operator Training

- ORR Activities 


\subsection{Work Breakdown Structure Index and Progammatic Responsibility Assignment Matrix}

\begin{tabular}{|c|c|c|c|c|c|}
\hline $\begin{array}{l}\text { Program } \\
\text { Element }\end{array}$ & Activity & $\begin{array}{c}\text { Cost } \\
\text { Account }\end{array}$ & Title & $\begin{array}{l}\text { Responsible } \\
\text { Manager }\end{array}$ & $\begin{array}{l}\text { Responsible } \\
\text { Organization }\end{array}$ \\
\hline \multirow[t]{11}{*}{ 1.2.1.1/A1 } & & & Solid Waste & & \\
\hline & $\mathrm{A} 12$ & $\begin{array}{l}1 \mathrm{~A} 1201 \\
1 \mathrm{~A} 1202 \\
1 \mathrm{~A} 1203 \\
1 \mathrm{~A} 1204 \\
1 \mathrm{~A} 1205 \\
1 \mathrm{~A} 1206\end{array}$ & RMW Treatment & D.E. McKenney & $\begin{array}{l}\text { Solid Waste } \\
\text { Programs }\end{array}$ \\
\hline & A19 & 1A1901 & $\begin{array}{l}\text { Systems } \\
\text { Engineering }\end{array}$ & D.E. McKenney & $\begin{array}{l}\text { Solid Waste } \\
\text { Programs }\end{array}$ \\
\hline & A1A & $\begin{array}{l}\text { 1A1A01 } \\
\text { 1A1A03 } \\
\text { 1A1A06 } \\
\text { 1A1A07 } \\
\text { 1A1A08 }\end{array}$ & Program Support & D.E. McKenney & $\begin{array}{l}\text { Solid Waste } \\
\text { Programs }\end{array}$ \\
\hline & AlM & $\begin{array}{l}\text { 1A1M01 } \\
\text { IAIM04 }\end{array}$ & $\begin{array}{l}\text { Management and } \\
\text { Training - } \\
\text { Programs }\end{array}$ & D.E. McKenney & $\begin{array}{l}\text { Solid Waste } \\
\text { Programs }\end{array}$ \\
\hline & ML2 & IML201 & Data Management & D.E. McKenney & $\begin{array}{l}\text { Solid Waste } \\
\text { Programs }\end{array}$ \\
\hline & A13 & $\begin{array}{l}1 \mathrm{~A} 1301 \\
1 \mathrm{~A} 1310\end{array}$ & TRU Storage & P.L. Hapke & $\begin{array}{l}\text { Solid Waste } \\
\text { Management }\end{array}$ \\
\hline & A14 & $\begin{array}{l}1 \mathrm{~A} 1401 \\
1 \mathrm{~A} 1410\end{array}$ & $\begin{array}{l}\text { RMW/CWC } \\
\text { Storage }\end{array}$ & P.L. Hapke & $\begin{array}{l}\text { Solid Waste } \\
\text { Management }\end{array}$ \\
\hline & A15 & $1 \mathrm{~A} 1501$ & $\begin{array}{l}\text { Hazardous Waste } \\
\text { Storage }\end{array}$ & R.D. Pierce & $\begin{array}{l}\text { Generator and } \\
\text { Waste Acceptance } \\
\text { Services }\end{array}$ \\
\hline & AlA & 1AtA05 & Solid Waste EIS & R.D. Pierce & $\begin{array}{l}\text { Generator and } \\
\text { Waste Acceptance } \\
\text { Services }\end{array}$ \\
\hline & A1M & 1A1M03 & $\begin{array}{l}\text { Management and } \\
\text { Training - GWAS }\end{array}$ & R.D. Pierce & $\begin{array}{l}\text { Generator and } \\
\text { Waste Accieptance } \\
\text { Services }\end{array}$ \\
\hline
\end{tabular}




\section{SOLID WASTE PROGRAM}

WBS 1.2.1

\begin{tabular}{|c|c|c|c|c|c|}
\hline & ML8 & 1ML801 & $\begin{array}{l}\text { Hazardous Waste } \\
\text { Receipt \& } \\
\text { Shipping }\end{array}$ & R.D. Pierce & $\begin{array}{l}\text { Generator and } \\
\text { Waste Acceptance } \\
\text { Services }\end{array}$ \\
\hline & A16 & $1 \mathrm{Al} 601$ & LLBG & P.L. Hapke & $\begin{array}{l}\text { Solid Waste } \\
\text { Management }\end{array}$ \\
\hline & A17 & $1 \mathrm{~A} 1701$ & $\begin{array}{l}\text { MW Trench } \\
\text { Storage }\end{array}$ & P.L. Hapke & $\begin{array}{l}\text { Solid Waste } \\
\text { Management }\end{array}$ \\
\hline & $2 \mathrm{~A} 14$ & $\begin{array}{l}2 \mathrm{~A} 1471 \\
2 \mathrm{~A} 1472\end{array}$ & CENRTC & P.L. Hapke & $\begin{array}{l}\text { Solid Waste } \\
\text { Management }\end{array}$ \\
\hline & $2 \mathrm{~A} 16$ & $2 \mathrm{~A} 1671$ & CENRTC & P.L. Hapke & $\begin{array}{l}\text { Solid Waste } \\
\text { Management }\end{array}$ \\
\hline & 2A1M & 2A1M72 & $\begin{array}{l}\text { Management and } \\
\text { Training }\end{array}$ & P.L. Hapke & $\begin{array}{l}\text { Solid Waste } \\
\text { Management }\end{array}$ \\
\hline & 5A14 & $5 A 1471$ & Small Projects & P.L. Hapke & $\begin{array}{l}\text { Solid Waste } \\
\text { Management }\end{array}$ \\
\hline & AlA & $\begin{array}{l}\text { 1A1A02 } \\
1 \mathrm{~A} 1 \mathrm{~A} 04\end{array}$ & Program Support & P.L. Hapke & $\begin{array}{l}\text { Solid Waste } \\
\text { Management }\end{array}$ \\
\hline & AlM & 1A1M02 & $\begin{array}{l}\text { Management and } \\
\text { Training - Solid } \\
\text { Waste }\end{array}$ & P.L. Hapke & $\begin{array}{l}\text { Solid Waste } \\
\text { Management }\end{array}$ \\
\hline \multirow[t]{6}{*}{$1.2 .1 .2 / \mathrm{A} 4$} & & & WRAP & & \\
\hline & A41 & $1 \mathrm{~A} 4101$ & Project Integration & S.J. Norton & $\begin{array}{l}\text { Solid Waste } \\
\text { Management }\end{array}$ \\
\hline & A46 & 1A4601 & ES\&QA & S.J. Norton & $\begin{array}{l}\text { Solid Waste } \\
\text { Management }\end{array}$ \\
\hline & A47 & $\begin{array}{l}\text { 1A4720 } \\
\text { 1A4721 } \\
\text { 1A4722 } \\
\text { 1A4724 } \\
\text { 1A4723 } \\
\text { 1A4725 }\end{array}$ & $\begin{array}{l}\text { WRAP } \\
\text { Operations/ } \\
\text { CENRTC }\end{array}$ & S.J. Norton & $\begin{array}{l}\text { Solid Waste } \\
\text { Management }\end{array}$ \\
\hline & A4M & 1A4M01 & $\begin{array}{l}\text { Management and } \\
\text { Training - WRAP }\end{array}$ & S.J. Norton & $\begin{array}{l}\text { Solid Waste } \\
\text { Management }\end{array}$ \\
\hline & $2 \mathrm{~A} 47$ & $2 A 4720$ & $\begin{array}{l}\text { WRAP } 1 \\
\text { CENRTC }\end{array}$ & S.J. Norton & $\begin{array}{l}\text { Solid Waste } \\
\text { Management }\end{array}$ \\
\hline
\end{tabular}


SOLID WASTE PROGRAM

WBS 1.2.1

EY 1997 Program Plan

WHC-SP-1114, REV 2

\begin{tabular}{|c|c|c|c|c|c|}
\hline 1.2.1.7/A3 & & & $\begin{array}{l}\text { Waste and } \\
\text { Decontamina- } \\
\text { tion Services }\end{array}$ & & \\
\hline & A31 & $1 \mathrm{~A} 3104$ & $\begin{array}{l}\text { Canyon } \\
\text { Processing }\end{array}$ & M.S. Wright & $\begin{array}{l}\text { Waste and } \\
\text { Decontamination } \\
\text { Services }\end{array}$ \\
\hline & A32 & $\begin{array}{l}1 \mathrm{~A} 3202 \\
1 \mathrm{~A} 3204\end{array}$ & $\begin{array}{l}\text { Low-Level } \\
\text { Processing }\end{array}$ & M.S. Wright & $\begin{array}{l}\text { Waste and } \\
\text { Decontamination } \\
\text { Services }\end{array}$ \\
\hline & A33 & $\begin{array}{l}1 A 3301 \\
1 A 3302 \\
1 A 3303 \\
1 A 3305 \\
1 A 3361 \\
1 A 3365\end{array}$ & $\begin{array}{l}\text { Contaminated } \\
\text { Equipment } \\
\text { Storage, GPP, and } \\
\text { CENRTC }\end{array}$ & M.S. Wright & $\begin{array}{l}\text { Waste and } \\
\text { Decontamination } \\
\text { Services }\end{array}$ \\
\hline & A34 & $1 \mathrm{~A} 3402$ & $\begin{array}{l}\text { Rail Car } \\
\text { Certification }\end{array}$ & M.S. Wright & $\begin{array}{l}\text { Waste and } \\
\text { Decontamination } \\
\text { Services }\end{array}$ \\
\hline & $\mathrm{A} 3 \mathrm{M}$ & 1А3M01 & $\begin{array}{l}\text { Management and } \\
\text { Training - T Plant }\end{array}$ & M.S. Wright & $\begin{array}{l}\text { Waste and } \\
\text { Decontamination } \\
\text { Services }\end{array}$ \\
\hline & $\mathrm{AB}$ & $1 \mathrm{AB} 101$ & $\begin{array}{l}\text { W-259 Project } \\
\text { Integration }\end{array}$ & M.S. Wright & $\begin{array}{l}\text { Waste and } \\
\text { Decontamination } \\
\text { Services }\end{array}$ \\
\hline & $3 \mathrm{AB}$ & $3 \mathrm{AB} 166$ & $\begin{array}{l}\text { W-259 Project } \\
\text { Construction }\end{array}$ & M.S. Wright & $\begin{array}{l}\text { Waste and } \\
\text { Decontamination } \\
\text { Services }\end{array}$ \\
\hline & $4 A 3$ & $4 A 3365$ & $\begin{array}{l}\text { FHA } \\
\text { Implementation }\end{array}$ & M.S. Wright & $\begin{array}{l}\text { Waste and } \\
\text { Decontamination } \\
\text { Services }\end{array}$ \\
\hline & $5 \mathrm{~A} 3$ & $5 A 3271$ & $\begin{array}{l}\text { 2706-T Free } \\
\text { Release Annex }\end{array}$ & M.S. Wright & $\begin{array}{l}\text { Waste and } \\
\text { Decontamination } \\
\text { Services }\end{array}$ \\
\hline \multirow[t]{2}{*}{ ML } & & & $\begin{array}{l}\text { Solid Waste } \\
\text { Assessments }\end{array}$ & & \\
\hline & ML2 & 1ML201 & Data Management & D.E. McKenney & $\begin{array}{l}\text { Solid Waste } \\
\text { Programs }\end{array}$ \\
\hline
\end{tabular}




\section{SOLID WASTE PROGRAM}

WBS 1.2.1

EY 1997 Propram Plan WHC-SP-1114. REV 2

\begin{tabular}{|l|l|l|l|l|l|}
\hline & ML4 & $1 \mathrm{ML402}$ & LLW Disposal & P.L. Hapke & $\begin{array}{l}\text { Solid Waste } \\
\text { Management }\end{array}$ \\
\hline & ML5 & 1 ML501 & TRU Receipt & P.L. Hapke & $\begin{array}{l}\text { Solid Waste } \\
\text { Management }\end{array}$ \\
\hline MD & ML6 & 1 ML603 & $\begin{array}{l}\text { RMW/CWC } \\
\text { Receipt }\end{array}$ & P.L. Hapke & $\begin{array}{l}\text { Solid Waste } \\
\text { Management }\end{array}$ \\
\hline & & Mazardous & & \\
Waste & $\begin{array}{l}\text { Hazardous Waste } \\
\text { Shipping and } \\
\text { Receiving }\end{array}$ & R.D. Pierce & $\begin{array}{l}\text { Generator and } \\
\text { Waste Acceptance } \\
\text { Services }\end{array}$ \\
\hline
\end{tabular}


Section 3.0

Schedule Baseline 


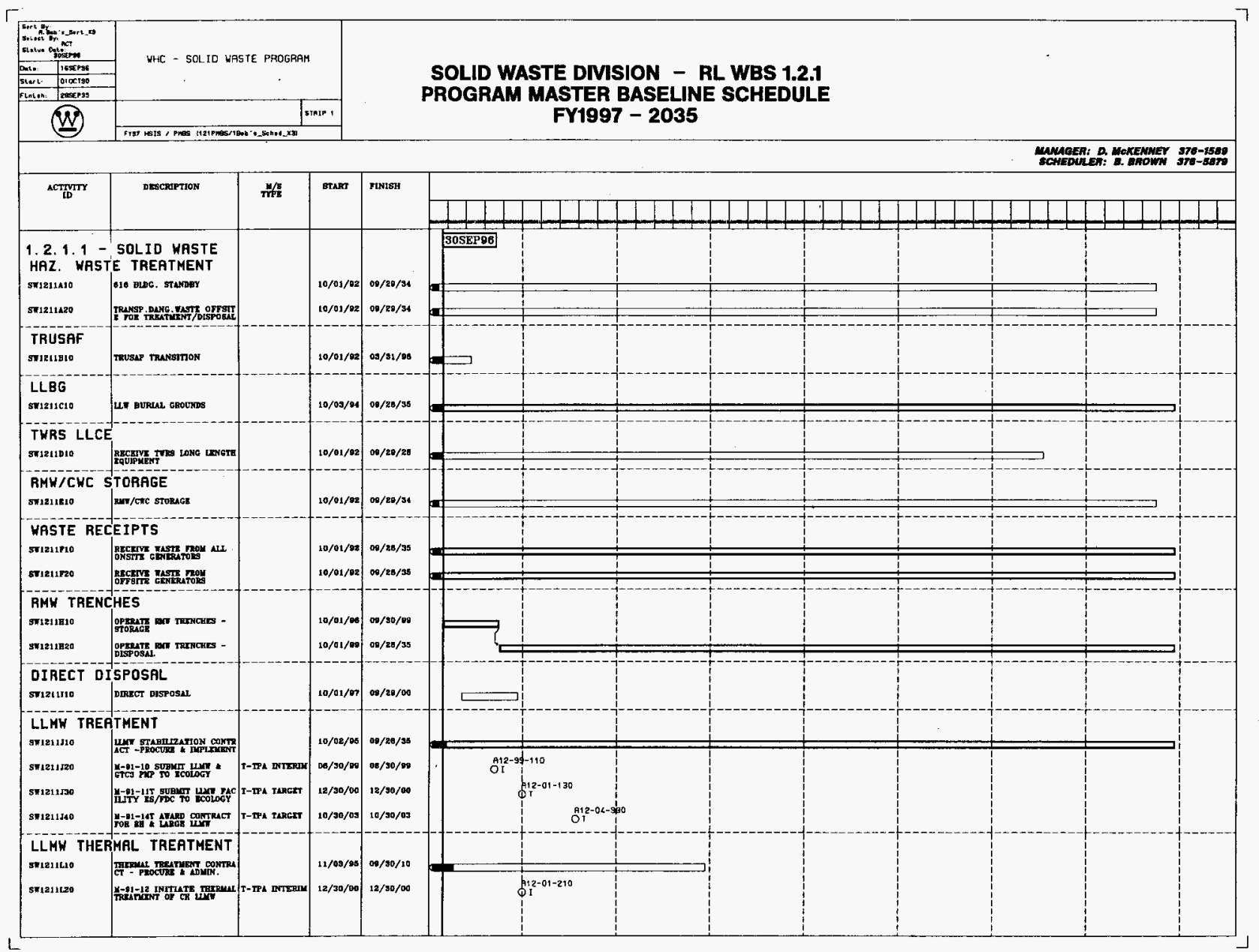




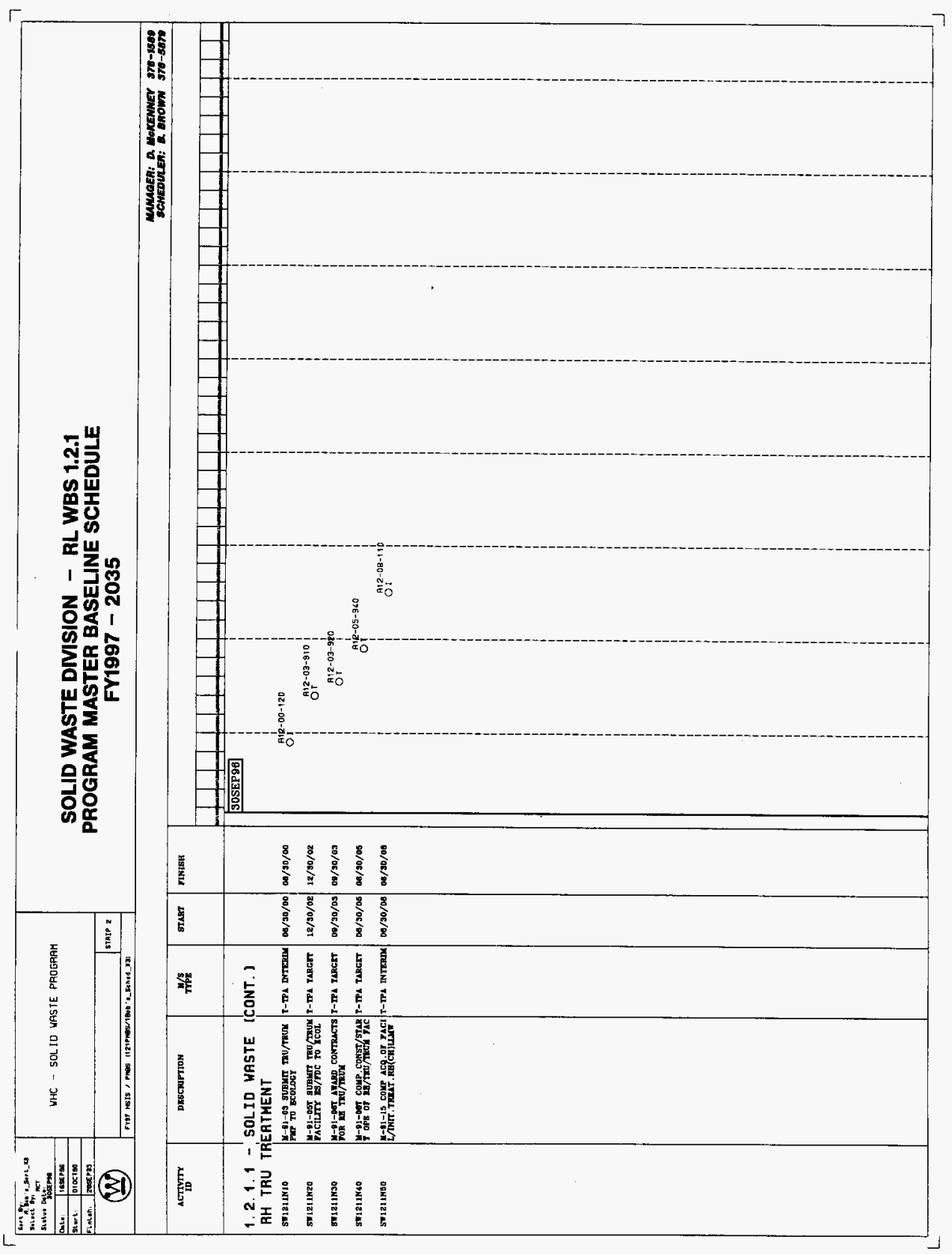




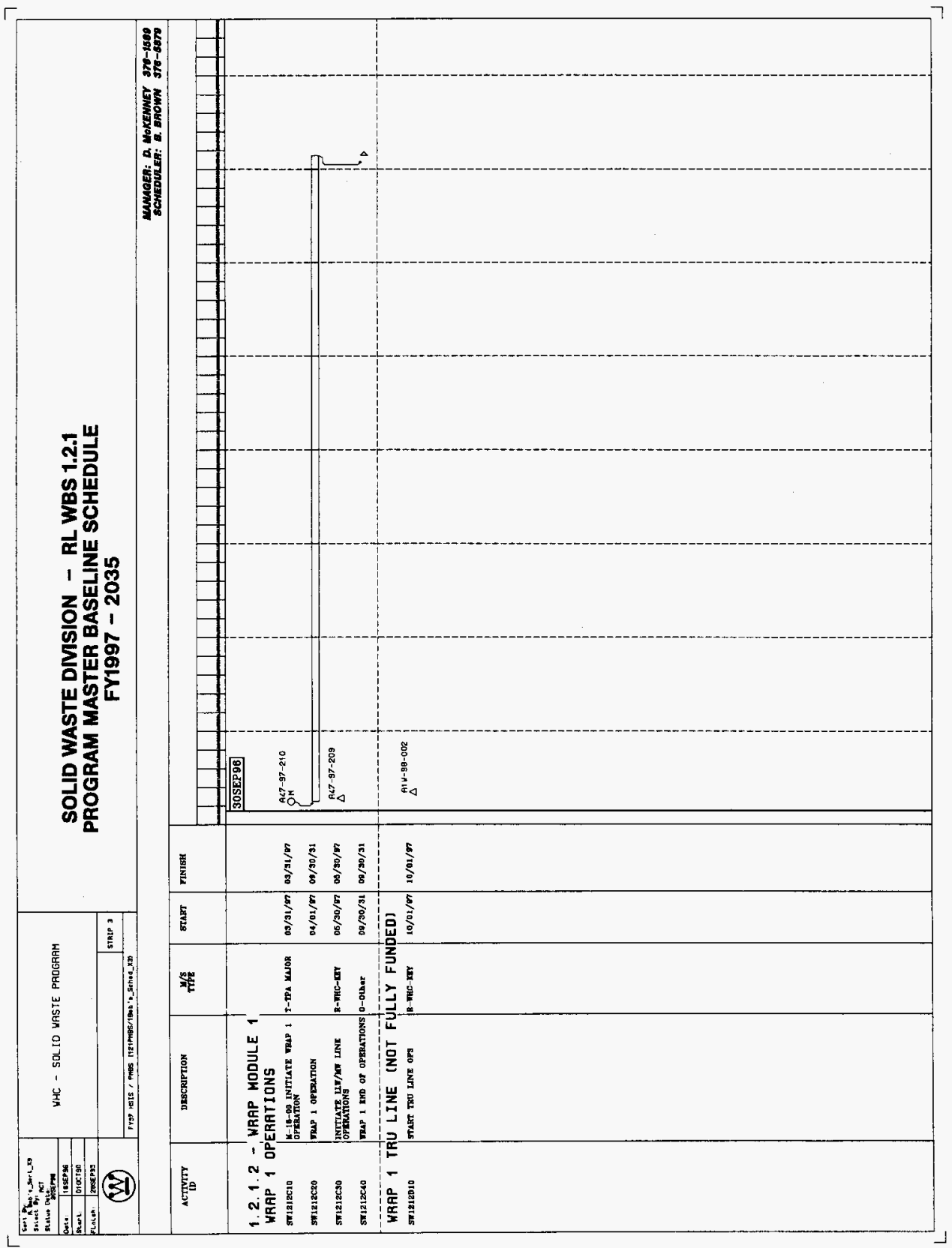




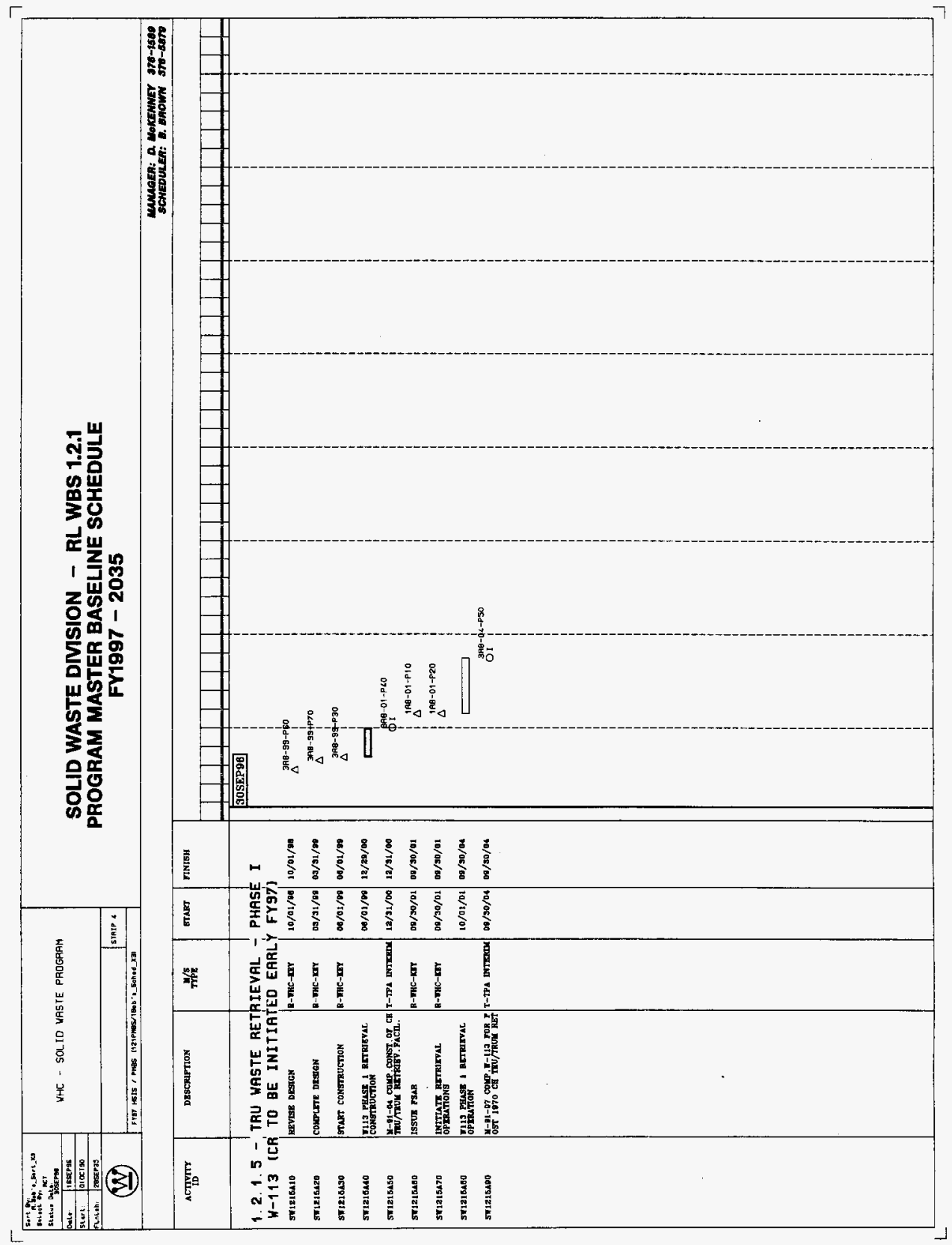




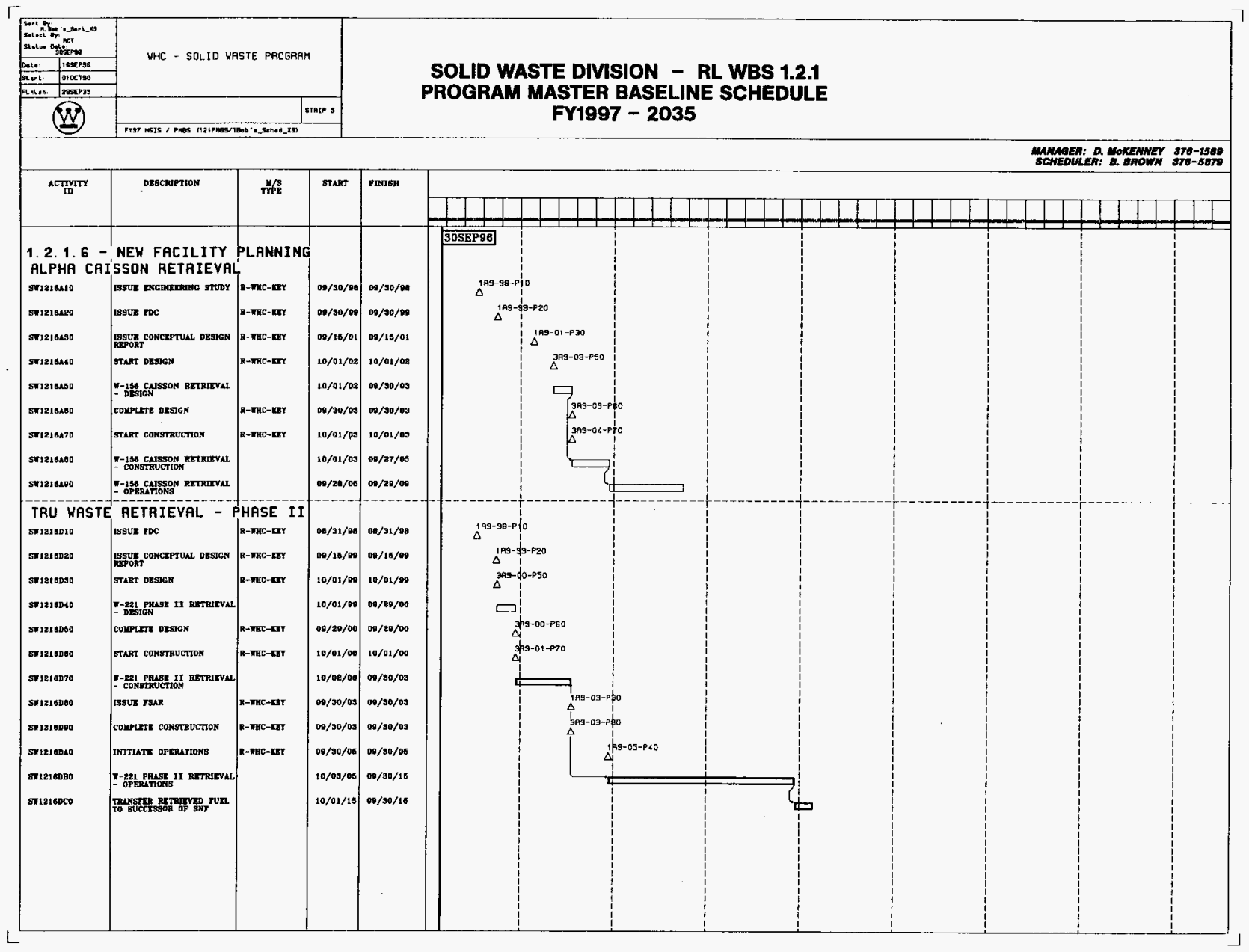




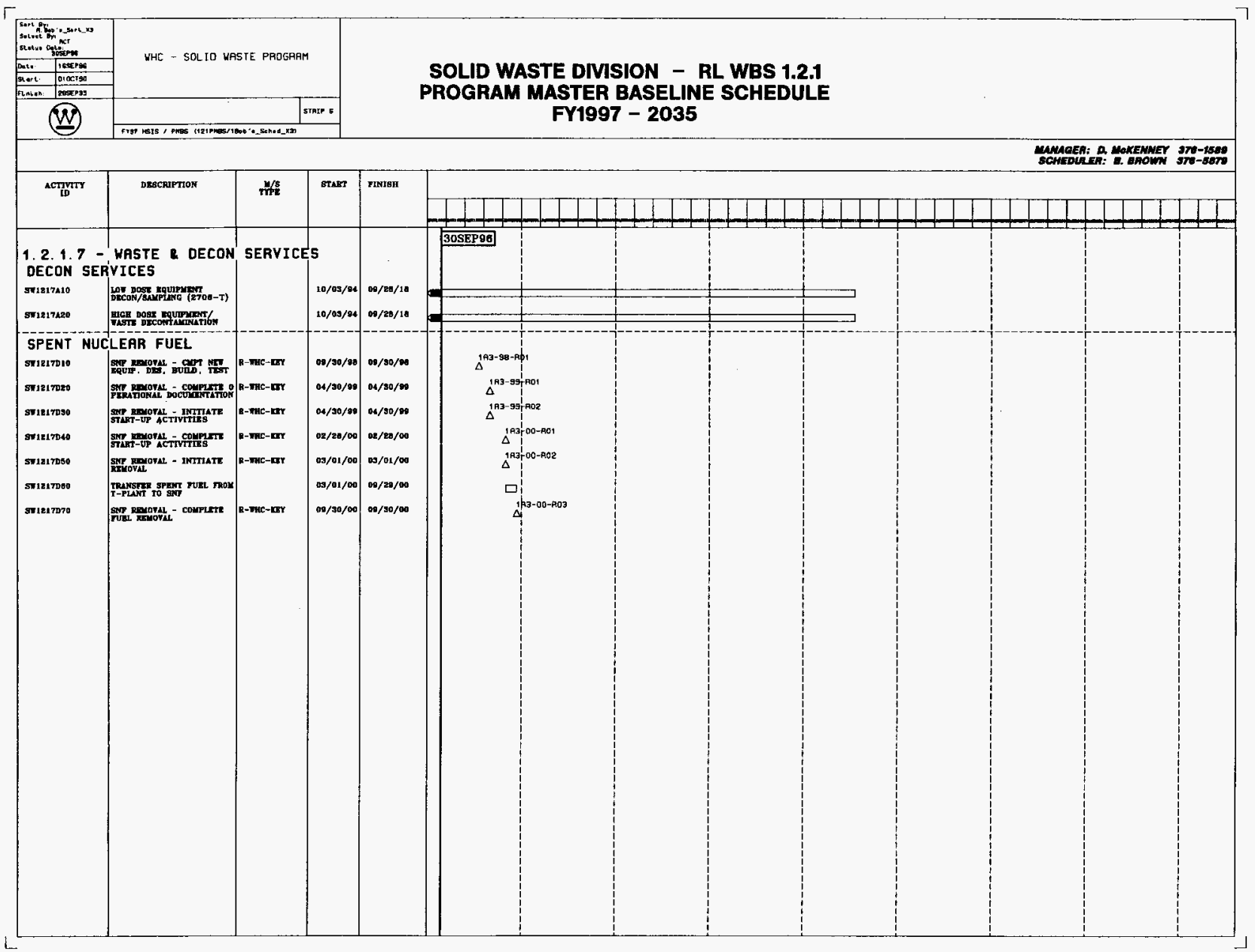




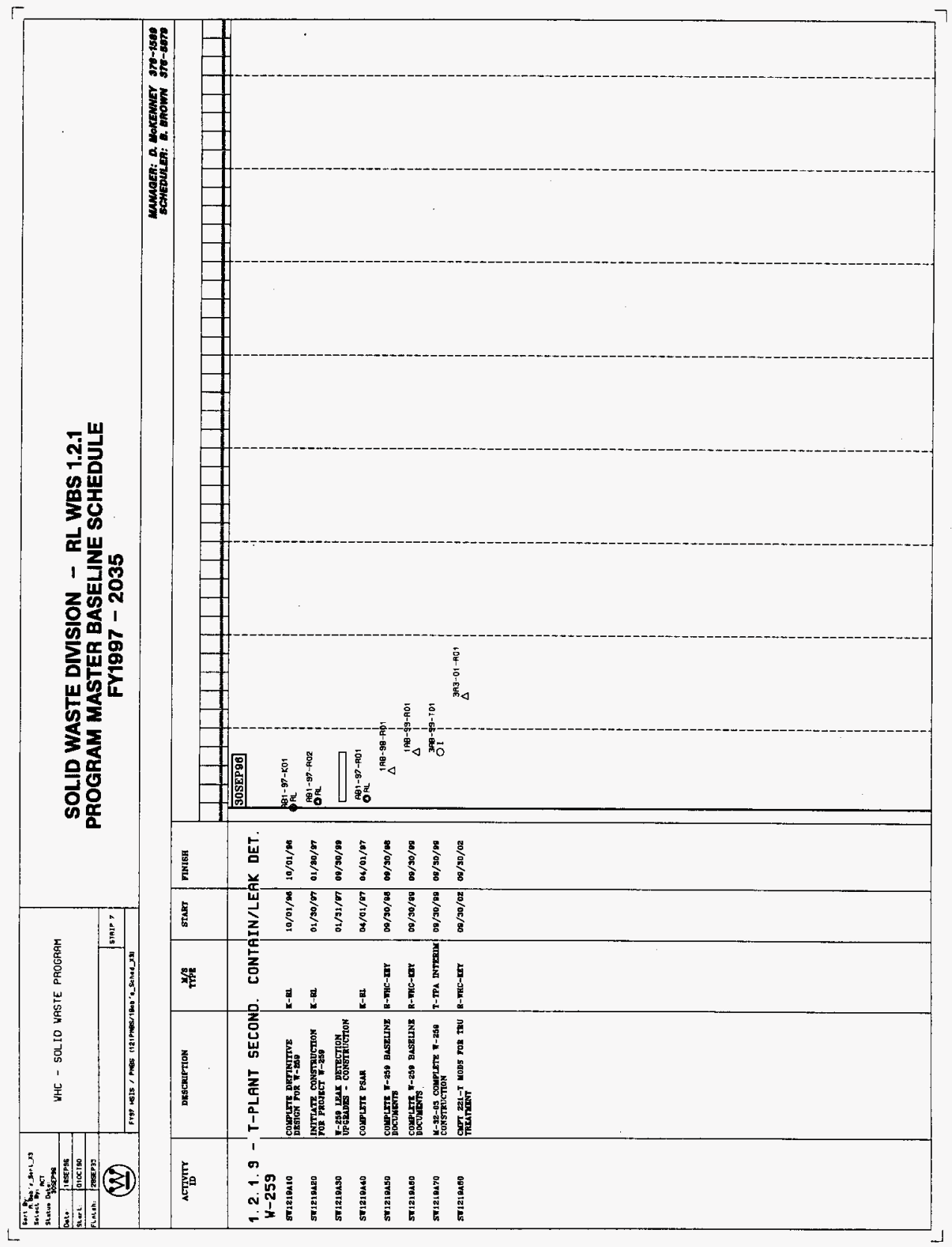




\begin{tabular}{|l|l|l|l||}
\hline $\begin{array}{c}\text { Milestone } \\
\text { Type }\end{array}$ & \multicolumn{1}{|c|}{$\begin{array}{c}\text { Control } \\
\text { Number }\end{array}$} & \multicolumn{1}{c|}{ Milestone Description } & $\begin{array}{c}\text { Milestone } \\
\text { Completion } \\
\text { Date }\end{array}$ \\
\hline RL & A12-97-015 & Complete Mixed Waste Treatment Technology Needs Report (WM 1.7.1) & 1/31/97 \\
\hline TPARL & A12-97-410 & Send Completion of Testing Notice to DOE (backlog soils) (TPA M-19-03) & $12 / 31 / 96$ \\
\hline TPARL & A14-97-101 & Initiate Operations at New CWC Slorage Facility (TPA M-91-09) & $6 / 1 / 97$ \\
\hline RL & A16-97-101 & Audits Show No Violation of Basis, PA, WAC (WM 1.6) & $9 / 30 / 97$ \\
\hline RL & A16-97-103 & Implement 200 Areas LLBG PA Maintenance Program (WM 1.8) & $6 / 30 / 97$ \\
\hline RL & A1A-97-010 & Submit Multi-Year Work Plan to DOE-RL & $7 / 30 / 97$ \\
\hline RL & A1M-97-P10 & Submit Site Waste Management Plan to DOE (WM 1.3.1) & $9 / 30 / 97$ \\
\hline RL & A1M-97-P20 & $\begin{array}{l}\text { Verify Quality Performance in Waste Managment to Industry Benchmarks } \\
\text { (WM 1.3.2) }\end{array}$ & $6 / 30 / 97$ \\
\hline RL & A31-97-K01 & Process Waste at Less than \$700/ft ${ }^{3}$ in the 221-T Facility (WM 1.4.1) & $9 / 30 / 97$ \\
\hline RL & A32-97-K03 & Process Waste at Less than \$120/ft ${ }^{3}$ in the 2706-T Facility (WM 1.5.1) & $9 / 30 / 97$ \\
\hline TPA/RL & A47-97-210 & Initiate WRAP 1 Operations (TPA M-18-00) (WM 1.1.1) & $3 / 31 / 97$ \\
\hline RL & AB1-97-R01 & Initiate Construction for Project W-259 (WM 1.2.1) & $1 / 30 / 97$ \\
\hline TPARL & A12-99-110 & M-91-10 Submit LLMW \& GTC3 PMP to Ecology (HAN-CJB-2) & $6 / 30 / 99$ \\
\hline \hline
\end{tabular}


SOLID WASTE PROGRAM

WBS 1.2.1

EY 1997 Program Plan

\begin{tabular}{||l|l|c|c||}
\hline $\begin{array}{c}\text { Milestone } \\
\text { Type }\end{array}$ & \multicolumn{1}{|c|}{$\begin{array}{c}\text { Control } \\
\text { Number }\end{array}$} & \multicolumn{1}{c|}{ Milestone Description } & $\begin{array}{c}\text { Milestone } \\
\text { Completion } \\
\text { Date }\end{array}$ \\
\hline TPA/RL & A12-00-120 & M-91-03 Submit TRU/TRUM PMP to Ecology & $6 / 30 / 00$ \\
\hline TPA/RL & A12-01-130 & M-91-11T Submit LLMW Facility ES/FDC to Ecology & $12 / 30 / 00$ \\
\hline TPA/RL & A12-01-210 & M-91-12 Initiate Thermal Treatment of CH LLMW & $12 / 30 / 00$ \\
\hline TPA/RL & A12-03-910 & M-91-05T Submit TRU/TRUM Facility ES/FDC to Ecology & $12 / 30 / 02$ \\
\hline TPA/RL & A12-03-920 & M-91-06T Award Contracts for RH TRU/TRUM & $9 / 30 / 03$ \\
\hline TPARL & A12-04-930 & M-91-14T Award Contracts for RH \& Large LLMW & $10 / 30 / 03$ \\
\hline TPA/RL & A12-05-940 & M-91-08T Comp. Constr./Start Ops. of RH/TRU/TRUM Facility & $6 / 30 / 05$ \\
\hline TPARL & $3 A 8-00-P 40^{*}$ & M-91-04 Comp. Constr. of CH TRU/TRUM Retrieval Facility & $9 / 30 / 00$ \\
\hline TPARL & $3 A 8-04-P 50$ & M-91-07 Comp. W-113 for Post 1970 CH TRU/TRUM Retrieval & $9 / 30 / 04$ \\
\hline
\end{tabular}

- Project W-113 planning does not reflect TPA M-91-04. A change request will be intiated in FY1997 to adjust outyear schedules and budget to meet the TPA M-91 milestones. 


\begin{tabular}{|c|c|c|c|}
\hline \multicolumn{4}{|c|}{$\begin{array}{l}\text { Westinghouse Hanford Company } \\
\text { MILESTONE DESCRIPTION SHEET }\end{array}$} \\
\hline \multicolumn{3}{|c|}{$\begin{array}{l}\text { Title: Complete Mixed Waste Treatment Technology Needs Report } \\
\text { (WM 1.7.1) }\end{array}$} & Date: $8 / 31 / 96$ \\
\hline \multicolumn{3}{|c|}{ Assigned To: D.E. McKEnney } & CIN: N/A \\
\hline \multicolumn{3}{|c|}{ Program WBS Designator: 1.2.1.1 } & Due Date:1/31/97 \\
\hline \multicolumn{3}{|c|}{ Control Number: A12-97-015 } & Rev: 0 \\
\hline $\begin{array}{l}\text { MILESTONE TYPE: } \\
\text { - DOE-HQ } \\
\text { X DOE-RL } \\
\text { - DOE-FO } \\
\text { - CONTRACTOR }\end{array}$ & $\begin{array}{l}\quad \text { DIVISION: } \\
\text { - State } \\
\text { - Federal } \\
\text { X DOE } \\
\text { - RCRA } \\
\text { - TPA Number }\end{array}$ & $\begin{array}{l}\text { DELIVERABLE: } \\
\text { X Report } \\
\text { - Letter } \\
\text { - Drawings } \\
\text { - Other (specify) }\end{array}$ & $\begin{array}{l}\text { ADDRESS TO: } \\
\text { - DOE-HQ } \\
\text { X DOE-RL } \\
\text { - Other (specify) }\end{array}$ \\
\hline \multicolumn{4}{|c|}{$\begin{array}{l}\text { Milestone Description: Complete a report which documents the technologies required to treat and } \\
\text { characterize waste in the SWD inventory. }\end{array}$} \\
\hline \multicolumn{4}{|c|}{ Description of what constitutes completion of this milestone: Completion of the report. } \\
\hline \multicolumn{2}{|c|}{ Cost Account Manager: Date } & \multicolumn{2}{|c|}{ Program/Project Manager Date } \\
\hline \multicolumn{2}{|c|}{ Program Element Manager } & \multicolumn{2}{|c|}{ DOE Monitor Date } \\
\hline
\end{tabular}




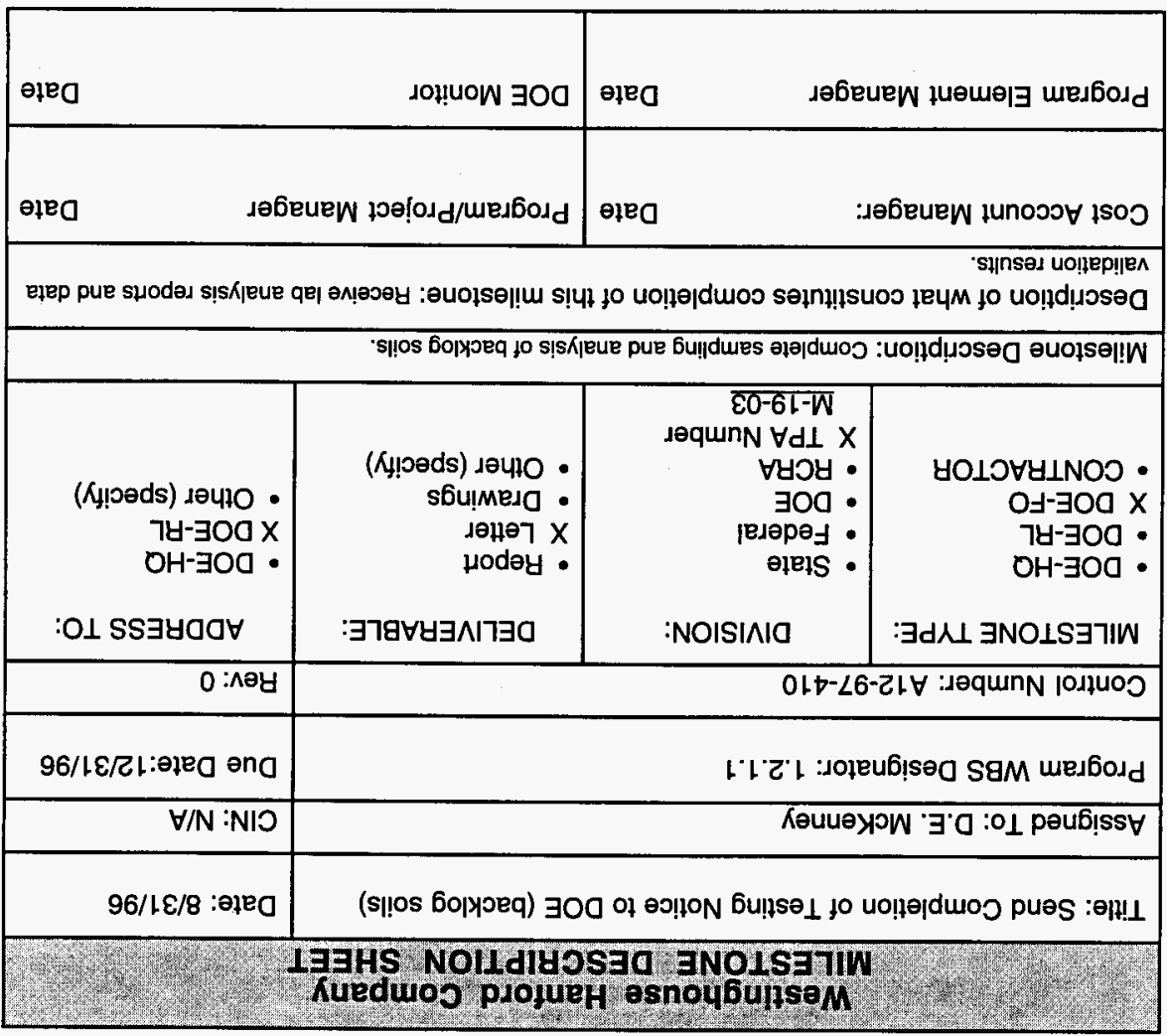




\begin{tabular}{|c|c|c|c|}
\hline (2) & $\begin{array}{l}\text { Westinghouse IHe } \\
\text { MLLESTONE DES }\end{array}$ & $\begin{array}{l}\text { anford Compan } \\
\text { GRIPTON SHE }\end{array}$ & 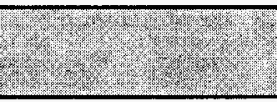 \\
\hline Title: Initiate Operatio & Is at New CWC Storage & Facility & Date: $8 / 31 / 96$ \\
\hline Assigned To: P.L. Ha & & & CIN: N/A \\
\hline Program WBS Design & ator: RL 1.2.1.1 & & Due Date: 6/01/97 \\
\hline Control Number: A14 & $97-101$ & & Rev: 0 \\
\hline $\begin{array}{l}\text { MILESTONE TYPE: } \\
\text { - DOE-HQ } \\
\text { - DOE-RL } \\
\text { X DOE-FO } \\
\text { - CONTRACTOR }\end{array}$ & $\begin{aligned} & \text { DIVISION: } \\
& \text { State } \\
& \text { - } \text { Federal } \\
& \text { - DOE } \\
& \text { - RCRA } \\
& \text { X TPA Number } \\
& \text { M-91-09 } \\
&\end{aligned}$ & $\begin{array}{l}\text { DELIVERABLE: } \\
\text { - Report } \\
X \text { Letter } \\
\text { - Drawings } \\
\text { - Other (specify) }\end{array}$ & $\begin{array}{l}\text { ADDRESS TO: } \\
\text { - DOE-HQ } \\
\text { X DOE-RL } \\
\text { - Other (specify) }\end{array}$ \\
\hline $\begin{array}{l}\text { Milestone Description: } \\
\text { storage of waste. }\end{array}$ & Initiate operations of the new & 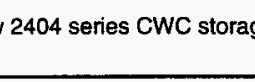 & acilities for long-term \\
\hline $\begin{array}{l}\text { Description of what cc } \\
\text { receive waste for long-term }\end{array}$ & $\begin{array}{l}\text { nstitutes completion of } t \\
\text { storage by letter to RL. }\end{array}$ & his milestone: Contrac & declaration of readiness to \\
\hline Cost Account Manage & Date & Program/Project $M$ & Date \\
\hline Program Element $\mathrm{Ma}$ & Date & DOE Monitor & Date \\
\hline
\end{tabular}




\begin{tabular}{|c|c|c|c|}
\hline \multirow{2}{*}{\multicolumn{2}{|c|}{ 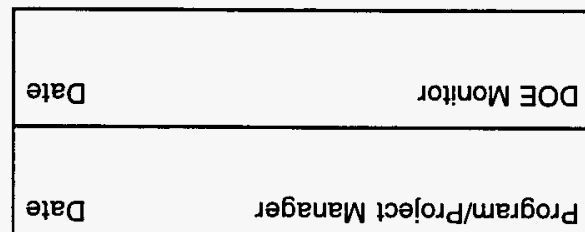 }} & \multicolumn{2}{|c|}{ 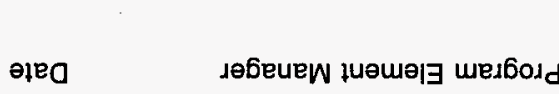 } \\
\hline & & \multicolumn{2}{|c|}{ :Jə6eueW lunosor $\forall$ lso } \\
\hline \multicolumn{4}{|c|}{ 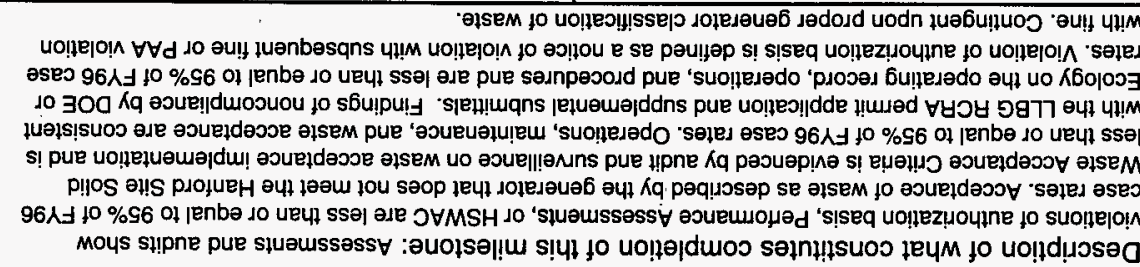 } \\
\hline \multicolumn{4}{|c|}{ 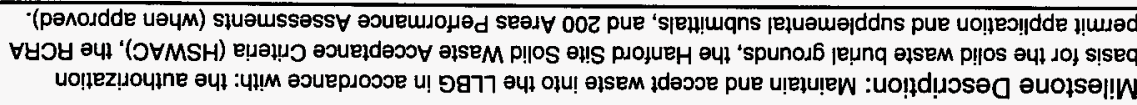 } \\
\hline 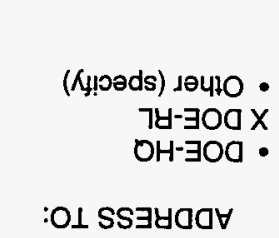 & 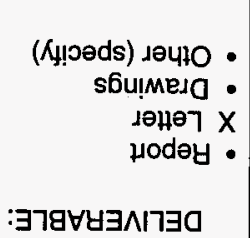 & 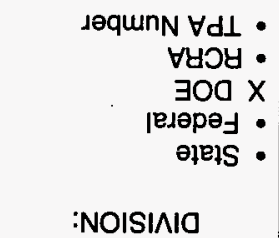 & 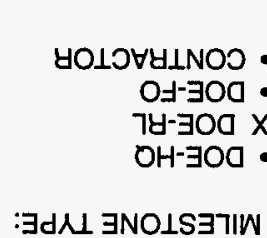 \\
\hline $0: \wedge \theta \mathrm{Y}$ & \multicolumn{3}{|c|}{ 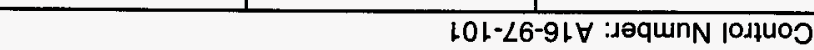 } \\
\hline L6/0ع/6:eleg əng & \multicolumn{3}{|c|}{ トトでト : גoןeu6!seg sgM wed6odd } \\
\hline$\forall / N: N I O$ & \multicolumn{3}{|c|}{ өxdeH ${ }^{\prime} T^{\prime} d: O_{\perp}$ peubịss } \\
\hline 96/LE/8 :өןe & \multicolumn{3}{|c|}{ 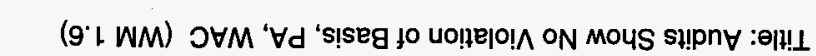 } \\
\hline$(-1)+1$ & HS Nolldith & $\begin{array}{l}\text { Ba } \\
\text { esnougursamin }\end{array}$ & (2) \\
\hline
\end{tabular}




\begin{tabular}{|c|c|c|c|}
\hline 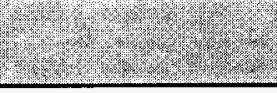 & $\begin{array}{l}\text { Westinghouse H } \\
\text { ILESTONE DES }\end{array}$ & $\begin{array}{l}\text { inford Compan } \\
\text { iPIPTION SHE }\end{array}$ & 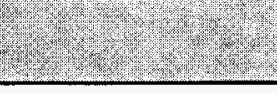 \\
\hline $\begin{array}{c}\text { Title: Implement } 200 \\
\text { (WM 1.8) }\end{array}$ & Areas LLBG PA Mainte & ance Program & Date: $8 / 31 / 96$ \\
\hline Assigned To: P.L. Ha & & & CIN: N/A \\
\hline Program WBS Desig & ator: 1.2.1.1 & & Due Date:6/30/97 \\
\hline Control Number: A16 & $97-103$ & & Rev: 0 \\
\hline $\begin{array}{l}\text { MILESTONE TYPE: } \\
\text { - DOE-HQ } \\
\text { X DOE-RL } \\
\text { - DOE-FO } \\
\text { - CONTRACTOR }\end{array}$ & $\begin{array}{l}\quad \text { DIVISION: } \\
\text { - State } \\
\text { - Federal } \\
\text { X DOE } \\
\text { - RCRA } \\
\text { - TPA Number }\end{array}$ & $\begin{array}{l}\text { DELIVERABLE: } \\
\text { - Report } \\
\text { X Letter } \\
\text { - Drawings } \\
\text { - Other (specify) }\end{array}$ & $\begin{aligned} & \text { ADDRESS TO: } \\
& \text { - } \text { DOE-HQ } \\
& \text { - } \text { DOE-RL } \\
& \text { - Other (specify) }\end{aligned}$ \\
\hline $\begin{array}{l}\text { Milestone Description } \\
\text { guidance. }\end{array}$ & stablish a maintenance $p$ & am for the 200 Area LL & PA in accordance with $\mathrm{HQ}$ \\
\hline $\begin{array}{l}\text { Description of what c } \\
\text { procedures to ensure that } \\
\text { PA is kept current thereaf }\end{array}$ & $\begin{array}{l}\text { istitutes completion of } t \\
\text { perations/waste accpetance } \\
\text { r. Confirmation to be sent to }\end{array}$ & $\begin{array}{l}\text { his milestone: DOE-R } \\
\text { are in compliance with th } \\
\text { DOE-RL by letter. }\end{array}$ & $\begin{array}{l}\text { pproved program plan and } \\
\text { A by June } 30,1997 \text { and }\end{array}$ \\
\hline Cost Account Manag & Date & Program/Project $M$ & Date \\
\hline Program Element Ma & Date & DOE Monitor & Date \\
\hline
\end{tabular}




\begin{tabular}{|l|l|}
\hline \multicolumn{2}{|c|}{ Westinghouse Hanford Company } \\
\hline Title: Submit Multi-Year Work Plan to DOE-RL
\end{tabular}




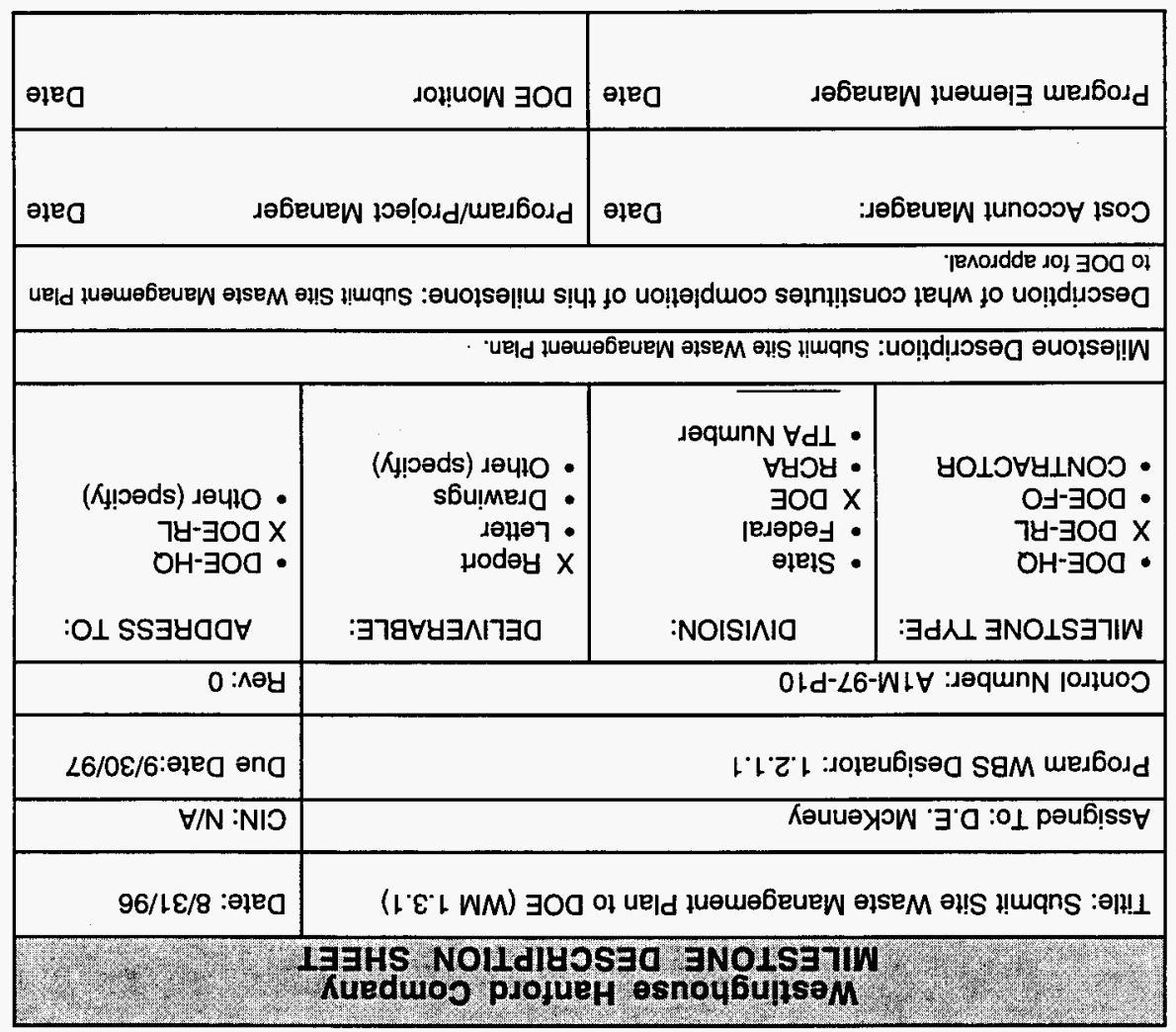




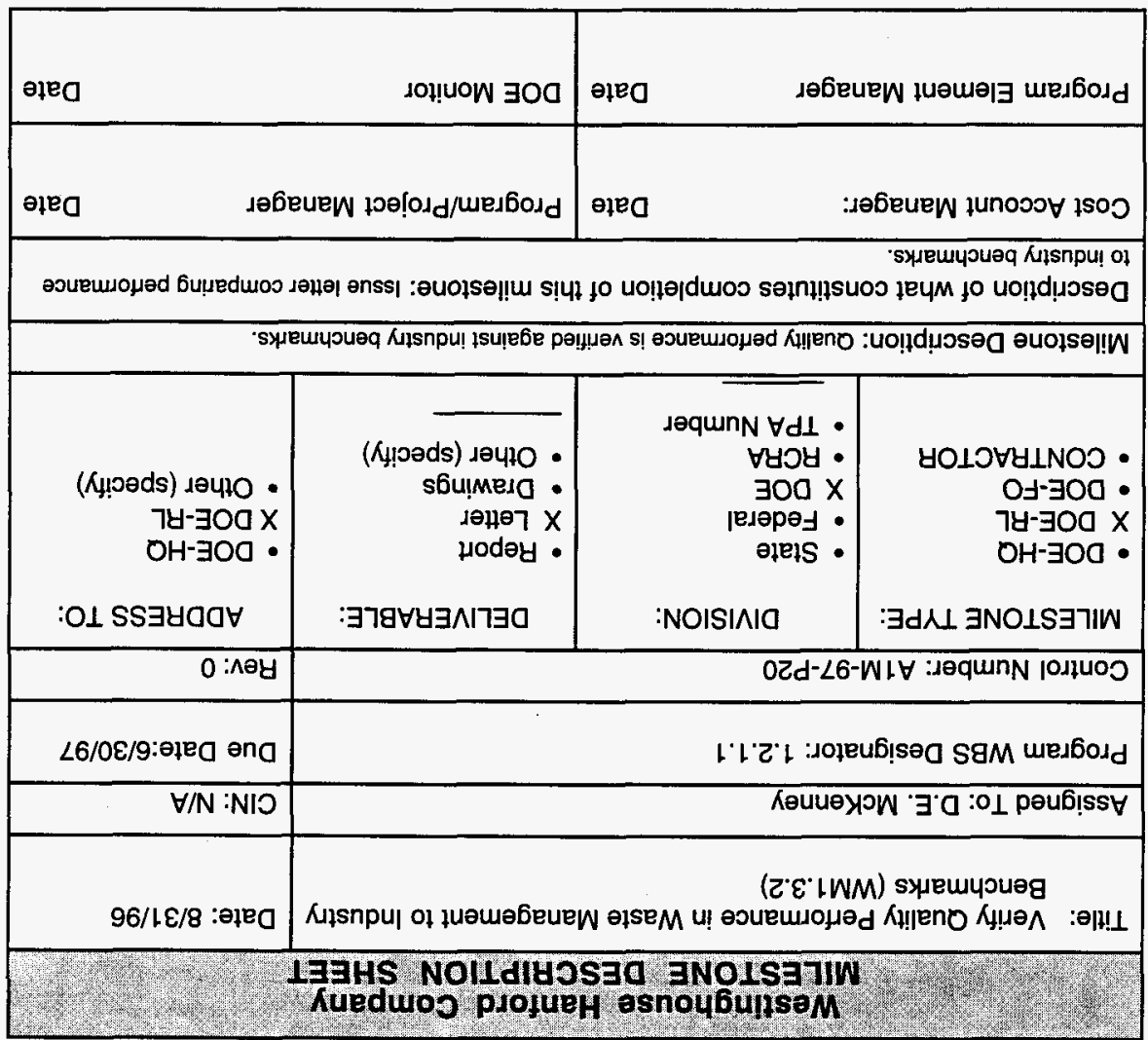




\begin{tabular}{|c|c|c|c|}
\hline Prate & $\begin{array}{l}\text { Westinghouse H } \\
\text { MILESTONE DES }\end{array}$ & $\begin{array}{l}\text { inford Compa } \\
\text { SBIPTION SH }\end{array}$ & $(2+2)+1$ \\
\hline $\begin{aligned} \text { Title: Process Waste } \\
\text { (WM 1.4.1) }\end{aligned}$ & at Less than $\$ 700 / \mathrm{t}^{3}$ in & the 221-T Facility & Date: $8 / 31 / 96$ \\
\hline Assigned To: W.S. A & & & CIN: N/A \\
\hline Program WBS Desig & ator: 1.2.1.7 & & Due Date:9/30/97 \\
\hline Control Number: A31 & $97-K 01$ & & Rev: 0 \\
\hline $\begin{array}{l}\text { MILESTONE TYPE: } \\
\text { - DOE-HQ } \\
\text { X DOE-RL } \\
\text { - DOE-FO } \\
\text { - CONTRACTOR }\end{array}$ & $\begin{array}{l}\quad \text { DIVISION: } \\
\text { - State } \\
\text { - Federal } \\
\text { X DOE } \\
\text { - RCRA } \\
\text { - TPA Number }\end{array}$ & $\begin{array}{l}\text { DELIVERABLE: } \\
\text { - Report } \\
\text { - Letter } \\
\text { - Drawings } \\
\text { X Other (specify) } \\
\text { SMS PTS Report }\end{array}$ & $\begin{array}{l}\text { ADDRESS TO: } \\
\text { - DOE-HQ } \\
\text { X DOE-RL } \\
\text { - Other (specify) }\end{array}$ \\
\hline $\begin{array}{l}\text { Milestone Description } \\
\text { for less than } \$ 700 \mathrm{ft}^{3} \text {. Dec } \\
\text { procedures and the "Hant } \\
\text { determined at the end of } \\
\text { processed is less than pla } \\
\text { assumptions to be used. }\end{array}$ & $\begin{array}{l}\text { Proces waste/equipment fo } \\
\text { ontamination is pertromed in } \\
\text { rd Site Solid Waste Acceptar } \\
\text { ee reporting period, from Oct } \\
\text { ned, the planned volume wil }\end{array}$ & $\begin{array}{l}\text { storage, disposal, and/o } \\
\text { accordance with approve } \\
\text { ce Criteria," WHC-EP-00 } \\
\text { ober } 1,1996 \text { to Septemb } \\
\text { be used for calculation. }\end{array}$ & $\begin{array}{l}\text { use in the T Plant Canyon } \\
\text { Plant operating } \\
\text { 4. Final cost will } \\
\text { 30, 1997. If volume } \\
\text { rent calculation }\end{array}$ \\
\hline $\begin{array}{l}\text { Description of what } \mathrm{C} \\
\text { documented in the Site } \mathrm{M}\end{array}$ & $\begin{array}{l}\text { nstitutes completion of } \\
\text { anagement System Report. }\end{array}$ & his milestone: Compl & of this milestone will be \\
\hline Cost Account Manag & Date & Program/Project $M$ & Date \\
\hline Program Element Me & nager & DOE Monitor & Date \\
\hline
\end{tabular}




\begin{tabular}{|c|c|c|c|}
\hline Pats & $\begin{array}{l}\text { Westinghouse H } \\
\text { MILESTONE DES }\end{array}$ & $\begin{array}{l}\text { anford Compan } \\
\text { CRIPTION SHE }\end{array}$ & 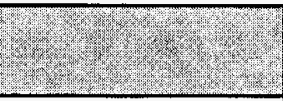 \\
\hline $\begin{array}{l}\text { Title: Process Waste } \\
\text { (WM 1.5.1) }\end{array}$ & at Less than $\$ 120 / \mathrm{ft}^{3}$ in & the 2706-T Facility & Date: $8 / 31 / 96$ \\
\hline Assigned To: W.S. Ay & & & CIN: N/A \\
\hline Program WBS Design & ator: 1.2.1.7 & & Due Date:9/30/97 \\
\hline Control Number: A32- & 97-KO3 & & Rev: 0 \\
\hline $\begin{array}{l}\text { MILESTONE TYPE: } \\
\text { - DOE-HQ } \\
\text { X DOE-RL } \\
\text { - DOE-FO } \\
\text { - CONTRACTOR }\end{array}$ & $\begin{array}{l}\quad \text { DIVISION: } \\
\text { - State } \\
\text { - Federal } \\
\text { X DOE } \\
\text { - RCRA } \\
\text { - TPA Number }\end{array}$ & $\begin{aligned} & \text { DELIVERABLE: } \\
& \text { - Report } \\
& \text { - Letter } \\
& \text { - Drawings } \\
& \text { X Other (specify) } \\
& \text { SMS PTS Report } \\
&\end{aligned}$ & $\begin{aligned} & \text { ADDRESS TO: } \\
& \text { - } \text { DOE-HQ } \\
& \text { X DOE-RL } \\
& \text { - Other (specify) }\end{aligned}$ \\
\hline $\begin{array}{l}\text { Milestone Description: } \\
\text { for less than } \$ 120 \mathrm{tt}^{3} \text {. Decc } \\
\text { procedures and the "Hanfo } \\
\text { determined at the end of th } \\
\text { processed is less than plar } \\
\text { assumptions to be used. }\end{array}$ & $\begin{array}{l}\text { Proces waste/equipment for } \\
\text { intamination is performed in } \\
\text { rd Site Solid Waste Acceptar } \\
\text { e reporting period, from Oct } \\
\text { ned, the planned volume will }\end{array}$ & $\begin{array}{l}\text { storage, disposal, and/o } \\
\text { accordance with approve } \\
\text { cce Criteria," WHC-EP-o0 } \\
\text { ober } 1,1996 \text { to Septemb } \\
\text { I be used for calculation. }\end{array}$ & $\begin{array}{l}\text { use in the } 2706-T \text { Facility } \\
\text { Plant operating } \\
\text { t. Final cost will } \\
0,1997 \text {. If volume } \\
\text { ent calculation }\end{array}$ \\
\hline $\begin{array}{l}\text { Description of what co } \\
\text { documented in the Site Ma }\end{array}$ & $\begin{array}{l}\text { nstitutes completion of } \\
\text { inagement System Report. }\end{array}$ & this milestone: Comple & of this milestone will be \\
\hline Cost Account Manage & Date & Program/Project $M$ & Date \\
\hline Program Element Mar & Date & DOE Monitor & Date \\
\hline
\end{tabular}




\begin{tabular}{|c|c|c|c|}
\hline \multicolumn{4}{|c|}{$\begin{array}{c}\text { Westinghouse Hanford company } \\
\text { MLESTONE DESCRIPTION SHEET }\end{array}$} \\
\hline \multicolumn{3}{|c|}{ Title: Initiate WAAP 1 Operations (WM 1.1.1) } & Date: $8 / 31 / 96$ \\
\hline \multicolumn{3}{|c|}{ Assigned To: S. H. Norton } & CIN: N/A \\
\hline \multicolumn{3}{|c|}{ Program WBS Designator: 1.2.1.1 } & Due Date:3/31/97 \\
\hline \multicolumn{3}{|c|}{ Control Number: A47-97-210 } & Rev: 0 \\
\hline $\begin{array}{l}\text { MILESTONE TYPE: } \\
\text { - DOE-HQ } \\
\text { - DOE-RL } \\
\text { X DOE-FO } \\
\text { - CONTRACTOR }\end{array}$ & $\begin{aligned} & \text { DIVISION: } \\
& \text { - State } \\
& \text { - } \text { Federal } \\
& \text { - DOE } \\
& \text { - RCRA } \\
& \text { X TPA Number } \\
& \text { M-18-00 } \\
&\end{aligned}$ & $\begin{array}{l}\text { DELIVERABLE: } \\
\text { - Report } \\
\text { - Letter } \\
\text { - Drawings } \\
\text { X Other (specify) } \\
\text { SMS PTS Report }\end{array}$ & $\begin{aligned} & \text { ADDRESS TO: } \\
& \text { - } \text { DOE-HQ } \\
& \text { X DOE-RL } \\
& \text { - Other (specify) }\end{aligned}$ \\
\hline \multicolumn{4}{|c|}{ Milestone Description: Initiate NDE/NDA and Waste Receipts at the WRAP 1 Facility. } \\
\hline \multicolumn{4}{|c|}{$\begin{array}{l}\text { Description of what constitutes completion of this milestone: Initiate NDE/NDA of suspect } \\
\text { TRU Waste at the WRAP } 1 \text { Facility by } 3 / 31 / 97 \text {. }\end{array}$} \\
\hline \multicolumn{2}{|c|}{ Cost Account Manager: $\quad$ Date } & \multicolumn{2}{|c|}{ Program/Project Manager Date } \\
\hline \multicolumn{2}{|c|}{ Program Element Manager Date } & \multicolumn{2}{|c|}{ DOE Monitor Date } \\
\hline
\end{tabular}




\begin{tabular}{|c|c|c|c|}
\hline (n) & $\begin{array}{l}\text { Westinghouse H } \\
\text { MLESTONE DES }\end{array}$ & $\begin{array}{l}\text { inford Compai } \\
\text { RIPTION SHE }\end{array}$ & 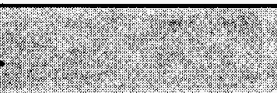 \\
\hline Title: Initiate Construc & ion for Project W-259 ( & WM 1.2.1) & Date: $8 / 31 / 96$ \\
\hline Assigned To: T. L. Ble & ckford & & CIN: N/A \\
\hline Program WBS Desigr & ator: 1.2.1.1 & & Due Date:1/30/97 \\
\hline Control Number: $A B 1$ & $97-R 01$ & & Rev: 0 \\
\hline $\begin{array}{l}\text { MILESTONE TYPE: } \\
\text { - DOE-HQ } \\
\text { X DOE-RL } \\
\text { - DOE-FO } \\
\text { - CONTRACTOR }\end{array}$ & $\begin{array}{l}\quad \text { DIVISION: } \\
\text { - State } \\
\text { - } \text { Federal } \\
\text { X DOE } \\
\text { - RCRA } \\
\text { - TPA Number }\end{array}$ & $\begin{array}{l}\text { DELIVERABLE: } \\
\text { - Report } \\
\text { X Letter } \\
\text { - Drawings } \\
\text { - Other (specify) }\end{array}$ & $\begin{aligned} & \text { ADDRESS TO: } \\
& \text { - } \text { DOE-HQ } \\
& \text { X DOE-RL } \\
& \text { - Other (specify) }\end{aligned}$ \\
\hline Milestone Description & Initiate procurement phase & of Project W-259 constru & \\
\hline $\begin{array}{l}\text { Description of what cc } \\
\text { specification to Procurem }\end{array}$ & $\begin{array}{l}\text { nstitutes completion of } \\
\text { nt to initiate procurement pro }\end{array}$ & $\begin{array}{l}\text { his milestone:Transmit } \\
\text { cess. }\end{array}$ & of procurement \\
\hline Cost Account Manag & Date & Program/Project $\mathrm{Ma}$ & Date \\
\hline Program Element Ma & nager & DOE Monitor & Date \\
\hline
\end{tabular}




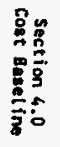




\section{SOLID WASTE PROGRAM \\ WBS 1.2.1}

FY 1997 Program Plan

\section{BUDGET AUTHORITY SUMMARY BY YEAR BY ADS}

WHC-SP-1114, REV. 2 (5000s)

\begin{tabular}{|c|c|c|c|c|c|c|c|c|c|c|c|c|c|}
\hline RL WBS \# & ADS : & TITLE & FY1997 & FY1998 & FY1999 & FY2000 & FY2001 & FY2002 & FY2003 & FY2004 & FY2005 & FY2006 & TOTAL. \\
\hline 1.2.1.1 & $2200-0$ & A1-Solid Waste & $28,605.6$ & $46,546.5$ & $55,796.0$ & $64,829.8$ & $78,272.0$ & 97.573 .8 & $102,762.4$ & $96,728.5$ & $107,495.8$ & $102,231,4$ & $780,841.9$ \\
\hline 1.2.1.2 & 2220-1 & A4-WRAP Module 1 & $10,705.4$ & & & & & & & & & & $10,705.4$ \\
\hline 1.2.1.5 & $2200-2$ & A8-Waste Retrieval & & 818.3 & $18,437.8$ & $13,258.2$ & $4,577.6$ & $6,153.4$ & $6,317.0$ & $6,487.7$ & & & $56,049.9$ \\
\hline 1.2 .1 .6 & $2250-0$ & A9-New Facility Planning & & 1.422 .7 & $4,158.0$ & $3,039.0$ & $16,875.0$ & $32,517.4$ & $26,367.9$ & $31,876.2$ & $26,696.8$ & $31,309.5$ & $174,262.5$ \\
\hline 1.2.1.7 & $2320-0$ & A3-Waste \& Decon Srve. & $21,418.8$ & $24,389.8$ & $25,309.4$ & $31,663.0$ & $34,029,4$ & $25,213,4$ & $26,010.7$ & $26,365.6$ & $27,265.4$ & $27,917.1$ & $269,582.5$ \\
\hline 1.2.1.9 & $2320-2$ & AB-T Plant 2nd Cont. & $4,770.1$ & $3,485.7$ & $2,616.8$ & & & & & & & & $10,872.6$ \\
\hline
\end{tabular}

(1) Summary Of All Programmatic ADS's And New B/A (See Exhibit 2); Does Not Include Expense Carryover. 


\section{SOLID WASTE PROGRAM}

WBS 1.2.1

FY 1997 Program Plan

BUDGET AUTHORITY BY YEAR BY ADS

Page 1 of 3

WHC-SP-1114, REV. 2

(\$000s)

\begin{tabular}{|c|c|c|c|c|c|c|c|c|c|c|c|c|c|c|}
\hline RL WBS \# & ADS \# & TITLE & FUND TYPE & FY1997 & FY1998 & FY1999 & FY2000 & FY2001 & FY2002 & FY2003 & FY2004 & FY2005 & FY2006 & TOTAL \\
\hline \multirow[t]{6}{*}{1.2 .1 .1} & $2200-0$ & A1-Solid Waste & Expense & $28,450.8$ & $44,463.9$ & $53,721.5$ & $61,500.7$ & $75,327.5$ & $94,413.4$ & $99,786.7$ & $93,529.9$ & $104,207.4$ & $98,858.7$ & $754,260.6$ \\
\hline & & & $\begin{array}{l}\text { CENRTC } \\
\text { Line Item }\end{array}$ & 157.1 & $1,426.2$ & 1,218.6 & $1,223.2$ & $1,256.3$ & $1,290.6$ & $1,325.0$ & $1,360.7$ & $1,397.6$ & 1.435 .3 & 12.090 .6 \\
\hline & & & GPP & 140.7 & 600.0 & $1,500.0$ & $1,539.2$ & $1,579.4$ & $1,621.2$ & $1,663.0$ & $1,706.5$ & $1,751.3$ & $1,790.0$ & $13,891.3$ \\
\hline & & & Subtotal New B/A & 28.748 .6 & $46,490.1$ & $56,440.1$ & $64,263.0$ & $78,163.2$ & $97,325.3$ & $102,774.6$ & $96,597.2$ & $107,356.3$ & $102,084.0$ & $780,242,4$ \\
\hline & & & Expense Carryover (1) & $2,334.1$ & & & & & & & & & & $2,334.1$ \\
\hline & & & Total B/A & $31,082.7$ & $46,490.1$ & 56.440 .4 & $64,263.0$ & $78,163.2$ & $97,325.3$ & $102,774.6$ & $96,597.2$ & $107,356.3$ & $102,084.0$ & 782.576 .5 \\
\hline \multirow[t]{4}{*}{1.2 .1 .2} & $2220-1$ & A4.WRAP Module 1 & Expense & $10,658.7$ & & & & & & & & & & $10,658.7$ \\
\hline & & & $\begin{array}{l}\text { Line Item } \\
\text { GPP }\end{array}$ & & & & & & & & & & & \\
\hline & & & $\begin{array}{l}\text { Subtotal New B/A } \\
\text { Expense Carryover (1) }\end{array}$ & $10,706.7$ & & & & & & & & & & \\
\hline & & & Total B/A & $10,706.7$ & & & & & & & & & & $10,706.7$ \\
\hline \multirow[t]{4}{*}{1.2 .1 .5} & $2200-2$ & AB-Waste Retrieval & $\begin{array}{l}\text { Expense } \\
\text { CENRTC }\end{array}$ & & 818.3 & $2,064.7$ & $\begin{array}{l}3,278.2 \\
1,633.5\end{array}$ & $4,577.6$ & 6.153 .4 & 6.317 .0 & $6,487.7$ & & & $\begin{array}{r}29,696.8 \\
1,633.5\end{array}$ \\
\hline & & & Line Item & & & $15,836.6$ & $4,893.1$ & & & & & & & $20,729.7$ \\
\hline & & & $\begin{array}{l}\text { Subtotal New B/A } \\
\text { Expense Carryover (1) }\end{array}$ & & 818.3 & $17,901.3$ & $9,804.7$ & $4,577.6$ & 6.153 .4 & 6.317 .0 & 6.487 .7 & & & $52,060.0$ \\
\hline & & & Total B/A & & 818.3 & $17,901,3$ & $9,804.7$ & $4,577.6$ & $6,153.4$ & $6,317.0$ & 6.487 .7 & & & $52,060.0$ \\
\hline
\end{tabular}


SOLID WASTE PROGRAM

WBS 1.2.1

FY 1997 Program Plan

BUDGET AUTHORITY BY YEAR BY ADS

Page 2 of 3

WHC-SP-1114, REV. 2

(\$000s)

\begin{tabular}{|c|c|c|c|c|c|c|c|c|c|c|c|c|c|c|}
\hline RL WBS * & ADS : & TITLE & FUND TYPE & FY1997 & FY1998 & FY1999 & FY2000 & FY2001 & FY2002 & FY2003 & FY2004 & FY2005 & FY2006 & TOTAL \\
\hline \multirow[t]{6}{*}{1.2 .1 .6} & $2250-0$ & A9-New Facility Planning & Expense & & 1.422 .7 & $4,158.0$ & $2,223.5$ & $2,876.9$ & $3,734.1$ & $2,648.6$ & $2,940.7$ & $4,125.7$ & $6,654.9$ & $30,755.1$ \\
\hline & (2) & & CENRTC & , & & & & 969.2 & $1,855.6$ & 993.0 & $1,019.9$ & $1,047.4$ & $2,550.1$ & $8,435.9$ \\
\hline & & & Line Item & & & & 4.581 .3 & $13,028.9$ & 26.927 .7 & $22,726.3$ & $27,945.7$ & $21,523.7$ & $22,104.5$ & $138,838.1$ \\
\hline & & & GPP & & & & & & & & & & & - \\
\hline & & & $\begin{array}{l}\text { Subtotal New B/A } \\
\text { Expense Carryover (1) }\end{array}$ & & $1,422.7$ & $4,158.0$ & $6,804.8$ & $16,875.0$ & $32,517.4$ & $26,367.9$ & $31,876.2$ & $26,696.8$ & $31,309.5$ & $178,028.3$ \\
\hline & & & Total $B / A$ & & 1.422 .7 & 4.158 .0 & 6.804 .8 & 16.875 .0 & $32,517,4$ & $26,367.9$ & $31,876.2$ & $26,696.8$ & $31,309.5$ & $178,028.3$ \\
\hline \multirow[t]{6}{*}{1.2 .1 .7} & $2320-0$ & A3-Waste \& Decon Srve & Expense & $19,022.1$ & $21,750.0$ & $22,426.8$ & $23,004.7$ & $22,422.8$ & $24,576.2$ & $25,089.1$ & $25,563.0$ & $26,445.7$ & $27,079.4$ & 237.379 .9 \\
\hline & & & CENRTC & & 796.0 & 817.4 & 839.5 & 862.2 & 885.8 & 909.4 & 933.9 & 959.2 & 985.1 & $7,988.4$ \\
\hline & & & Line Item & & & & $7,920.4$ & $10,853.3$ & & & & & & $18,773.7$ \\
\hline & & & GPP & $2,331.8$ & $1,900.2$ & $1,957.5$ & 152.8 & & & & & & & $6,342.3$ \\
\hline & & & $\begin{array}{l}\text { Subtotal New B/A } \\
\text { Expense Carryover (1) }\end{array}$ & $21,353.9$ & $24,446.1$ & $25,201.8$ & $31,917.5$ & $34,138.2$ & $25,462.0$ & $25,998.5$ & $26,496.9$ & $27,404.9$ & $28,064.5$ & 270.484 .3 \\
\hline & & & Total $B / A$ & $21,353.9$ & $24,446.1$ & $25,201.8$ & $31,917.5$ & $34,138.2$ & $25,462.0$ & $25,998.5$ & $26,496.9$ & $27,404.9$ & $28,064.5$ & $270,484.3$ \\
\hline \multirow[t]{4}{*}{ 1.2.1.9 } & $2320-2$ & AB-T Plant 2nd Cont & $\begin{array}{l}\text { Expense } \\
\text { CENRTC }\end{array}$ & 690.8 & 785.8 & 717.2 & & & & & & & & $\begin{array}{c}2.193 .8 \\
-\end{array}$ \\
\hline & & & $\begin{array}{l}\text { Line Item } \\
\text { GPP }\end{array}$ & $3,999.9$ & $2,700.0$ & $1,899.6$ & & & & & & & & $8,599.5$ \\
\hline & & & $\begin{array}{l}\text { Subtotal New B/A } \\
\text { Expense Carryover ( } t \text { ) }\end{array}$ & $4,690.8$ & $3,485.8$ & $2,616.8$ & & & & & & & & $10,793.3$ \\
\hline & & & Total B/A & $4,690.8$ & $3,485.8$ & $2,616.8$ & & & & & & & & $10,793.3$ \\
\hline
\end{tabular}


SOLID WASTE PROGRAM

WBS 1.2.1

FY 1997 Program Plan

BUDGET AUTHORITY BY YEAR BY ADS

Page 3 of 3

WHC-SP-1114, REV. 2

(\$000s)

\begin{tabular}{|c|c|c|c|c|c|c|c|c|c|c|c|c|c|c|}
\hline RL WBS \# & ADS \# & TITLE & FUND TYPE & FY1997 & FY1998 & FY1999 & FY2000 & FY2001 & FY2002 & FY2003 & FY2004 & FY2005 & FY2006 & TOTAL \\
\hline \multirow[t]{7}{*}{1.2 .1} & All Direct & Solid Waste Program & Expense & $58,822.4$ & $69,240.7$ & $83,088.2$ & $90,007.1$ & $105,204.7$ & $128,877.1$ & $133,841.5$ & $128,491.3$ & $134,778.8$ & $132,593.0$ & $1,064,944.8$ \\
\hline & & & CENRTC & 205.2 & $2,222.1$ & $2,036.0$ & $3,696.2$ & $3,087.7$ & $4,032.0$ & $3,227.3$ & $3,314.5$ & $3,404.2$ & $4,970.4$ & $30,195.7$ \\
\hline & & & Line Item & $3,999.9$ & $2,700.0$ & 17.736 .2 & $17,394.8$ & $23,882.1$ & 26.927 .7 & $22,726.3$ & $27,945.7$ & $29,523.7$ & $22,104.5$ & $186,940.9$ \\
\hline & & & GPP & $2,472.5$ & $2,500.2$ & $3,457.5$ & $1,692.0$ & $1,579.4$ & $1,621.2$ & $1,663.0$ & $1,706.5$ & $1,751.3$ & $1,790.0$ & $20,233.6$ \\
\hline & & & Subtotal New B/A & $65,500.0$ & $76,663.0$ & $106,318.0$ & $112,790.0$ & $133,754.0$ & $161,458.0$ & $161,458.0$ & $161,458.0$ & $161,458.0$ & $161,458.0$ & $1,302,315.0$ \\
\hline & & & Expense Carryover (1) & $2,334.1$ & & & & & & & & & & $2,334.1$ \\
\hline & & & Total B/A & $67,834.1$ & $76,663.0$ & $106,318.0$ & $112,790.0$ & $133,754.0$ & $161,458.0$ & $161,458.0$ & $161,458.0$ & $161,458.0$ & $161,458.0$ & $1,304,649.1$ \\
\hline
\end{tabular}

\begin{tabular}{|c|c|c|c|c|c|c|c|c|c|c|c|}
\hline PROGRAM NEW B/A & $65,500.0$ & $76,663.0$ & $106,318.0$ & $112,790.0$ & $133,754,0$ & $161,458.0$ & $161,458.0$ & $161,458.0$ & $161,458.0$ & $161,458.0$ & $1,302,315,0$ \\
\hline PROGRAM EXPENSE CARRYOVER & 2,334.1 & & & & & & & & & & $2,334.1$ \\
\hline PROGRAM TOTAL B/A & $67,834.1$ & $76,663.0$ & $106,318.0$ & $112,790.0$ & $133,754.0$ & $161,458.0$ & $161,458.0$ & $161,458.0$ & $161,458.0$ & $161,458.0$ & $1,304,649.1$ \\
\hline
\end{tabular}

(1) Includes Only Expense Carryover Approved By Site Management Board (SMB) Prior To 10/1/96. 


\section{SOLID WASTE PROGRAM}

WBS 1.2.1

FY 1997 Program Plan

\section{COST BASELINE BY YEAR BY ADS}

Page 1 of 3

(\$000s)

\begin{tabular}{|c|c|c|c|c|c|c|c|c|c|c|c|c|c|c|}
\hline RL WBS \# & ADS \# & TITLE & FUND TYPE & FY1997 & FY1998 & FY1999 & FY2000 & FY2001 & FY2002 & FY2003 & FY2004 & FY2005 & FY2006 & TOTAL \\
\hline \multirow[t]{5}{*}{1.2 .1 .1} & $2200-0$ & A1-Solid Waste & Expense & $30,784.9$ & 44.463 .9 & $53,721.5$ & $61,500.7$ & $75,327.5$ & $94,413.4$ & $99,786.7$ & $93,529.9$ & $104,207.4$ & $98,858.7$ & $756,594.6$ \\
\hline & & & $\begin{array}{l}\text { CENRTC } \\
\text { Line Item }\end{array}$ & 297.1 & $1,426.2$ & $1,218.6$ & $1,223.2$ & $1,256.3$ & $1,290.6$ & $1,325.0$ & $1,360.7$ & $1,397.6$ & $1,435.3$ & $12,230.6$ \\
\hline & & & GPP & 140.7 & 600.0 & $1,500.0$ & $1,539.2$ & $1,579.4$ & $1,621.2$ & $1,663.0$ & $1,706.5$ & $1,751.3$ & $1,790.0$ & $13,891.3$ \\
\hline & : & & $\begin{array}{l}\text { Total BCWS/PMB (1) } \\
\text { Mgmt Reserve (2) } \\
\text { Line Item Contingency (2) }\end{array}$ & $31,222.7$ & 46.490 .1 & 56.440 .1 & $64,263.1$ & $78,163.2$ & $97,325.2$ & $102,774.6$ & $96,597.2$ & $107,356.3$ & $102,084.0$ & 4500 \\
\hline & & & Total & $31,672.7$ & $46,490.1$ & $56,440.1$ & $64,263.1$ & 78.163 .2 & 97.325 .2 & 102.774 .6 & $96,597.2$ & $107,356.3$ & $102,084.0$ & $783,166.5$ \\
\hline \multirow[t]{5}{*}{1.2 .1 .2} & $2220-1$ & A4-WRAP Module 1 & Expense & $10,658.7$ & & & & & & & & & & $10,658.7$ \\
\hline & & & $\begin{array}{l}\text { Line Item } \\
\text { GPP }\end{array}$ & & & & & & & & & & & \\
\hline & & & Total BCWSPMM (1) & 10.706 .7 & & & & & & & & & & $10,706.7$ \\
\hline & & & $\begin{array}{l}\text { Line Item Contingency (2) } \\
\text { Expected Carryover (3) }\end{array}$ & & & & & & & & & & & \\
\hline & & & Total & $10,706.7$ & & & & & & & & & & $10,706.7$ \\
\hline \multirow[t]{5}{*}{ 1.2.1.5 } & $2200-2$ & A8-Waste Retrieval & $\begin{array}{l}\text { Expense } \\
\text { CENRTC }\end{array}$ & & 818.3 & $2,064.7$ & $\begin{array}{l}3,278.2 \\
1,633.5\end{array}$ & $4,577,6$ & $6,153.4$ & $6,317.0$ & $6,467.7$ & & & $\begin{array}{r}29,696.8 \\
1633.5\end{array}$ \\
\hline & & & Line Item & & & $15,836.6$ & $4,893.1$ & & & & & & & $20,729.7$ \\
\hline & & & Total BCWS/PMB (1) & & 818.3 & $17,901.3$ & $9,804.7$ & $4,577.6$ & $6,153.4$ & $6,317.0$ & 6.487 .7 & & & $52,060.0$ \\
\hline & & & $\begin{array}{l}\text { Mgmt Reserve (2) } \\
\text { Line Item Contingency (2) } \\
\text { Expected Carryover (3) }\end{array}$ & & & & & & & & & & & \\
\hline & & & Total & & 818.3 & $17,901,3$ & $9,804.7$ & $4,577.6$ & $6,153.4$ & 6.317 .0 & $6,487.7$ & & & $52,060.0$ \\
\hline
\end{tabular}


FY 1997 Program Plan

\section{SOLID WASTE PROGRAM}

WBS 1.2.1

COST BASELINE BY YEAR BY ADS

Page 2 of 3

(\$000s)

\begin{tabular}{|c|c|c|c|c|c|c|c|c|c|c|c|c|c|c|}
\hline RL WBS \# & ADS \# & TITLE & FUND TYPE & FY1997 & FY1998 & FY1999 & Fr2000 & FY2001 & FY2002 & FY2003 & FY2004 & FY2005 & FY2006 & TOTAL \\
\hline \multirow[t]{6}{*}{1.2 .1 .6} & $2250-0$ & A9-New Facility Planning & Expense & & $1,422.7$ & $4,158.0$ & $2,223.5$ & $2,876.9$ & $3,734.1$ & $2,648.6$ & $2,910.7$ & $4,125.7$ & $6,654.9$ & $30,755.1$ \\
\hline & & & CENRTC & & & & & 969.2 & $1,855.6$ & 993.0 & $1,019.9$ & $1,047.4$ & $2,550.1$ & $8,435.1$ \\
\hline & & & Line Item & & & & $4,581.3$ & $13,028.9$ & 26.927 .7 & 22.726 .3 & $27,945.7$ & $21,523.7$ & $22,104.5$ & $138,838.1$ \\
\hline & & & GPP & & & & & & & 263679 & & & 313095 & $\frac{\cdot}{1780283}$ \\
\hline & & & $\begin{array}{l}\text { Total BCWS/PMB (1) } \\
\text { Mgmt Reserve (2) } \\
\text { Line Item Contingency (2) } \\
\text { Expected Carryover (3) }\end{array}$ & & $1,422.7$ & $4,158.0$ & $6,804.8$ & $16,875.0$ & $32,517.4$ & $26,367.9$ & $31,876.2$ & $26,696.8$ & $31,309.5$ & $178,028.3$ \\
\hline & & & Total & & $1,422.7$ & $4,158.0$ & $6,804,8$ & $16,875.0$ & $32,517.4$ & 26.367 .9 & $31,876.2$ & $26,696.8$ & $31,309.5$ & $178,028.3$ \\
\hline \multirow[t]{6}{*}{ 1.2.1.7 } & $2320-0$ & A3-Waste \& Decon Srve & Expense & $19,022.1$ & $21,750.0$ & $22,426.8$ & $23,004.7$ & $22,422.8$ & $24,576.2$ & $25,089.1$ & $25,563.0$ & $26,445.7$ & $27,079.4$ & $237,379.9$ \\
\hline & & & CENRTC & 220.0 & 796.0 & 817.4 & 839.5 & 862.2 & 885.8 & 909.4 & 933.9 & 959.2 & 985.1 & $8,208.4$ \\
\hline & & & Line Item & & & & $7,920.4$ & 10.853 .3 & & & & & & 18.773 .7 \\
\hline & & & GPP & $2,931.8$ & $1,900.2$ & $1,957.5$ & 152.8 & & & & & & & $6,942.3$ \\
\hline & & & $\begin{array}{l}\text { Total BCWS/PMB (1) } \\
\text { Mgmt Reserve (2) } \\
\text { Line Item Contingency (2) } \\
\text { Expected Carryover (3) }\end{array}$ & $22,173.9$ & $24,446.1$ & $25,20 \leqslant .8$ & $31,917.5$ & $34,138.2$ & $25,462.0$ & $25,998.5$ & $26,496.9$ & $27,404.9$ & $28,064.5$ & $271,304.3$ \\
\hline & & & Total & $22,173.9$ & $24,446.1$ & $25,201.8$ & $31,917.5$ & $34,138.2$ & $25,462.0$ & $25,998.5$ & $26,496.9$ & $27,404.9$ & $28,064.5$ & $271,304.3$ \\
\hline \multirow[t]{5}{*}{1.2 .1 .9} & $2320-2$ & AB-T Plant 2nd Cont & $\begin{array}{l}\text { Expense } \\
\text { CENRTC }\end{array}$ & 690.8 & 785.8 & 717.2 & & & & & & & & $2,193.8$ \\
\hline & & & $\begin{array}{l}\text { Line Item } \\
\text { GPP }\end{array}$ & 4.099 .9 & $2,700.0$ & $1,899.6$ & & & & & & & & $8,699.5$ \\
\hline & & & $\begin{array}{l}\text { Total BCWS/PMB (1) } \\
\text { Mgmt Reserve (2) }\end{array}$ & $4,790.8$ & $3,485.7$ & $2,616.8$ & & & & & & & & $10,893.3$ \\
\hline & & & $\begin{array}{l}\text { Line Item Contingency (2) } \\
\text { Expected Carryover (3) }\end{array}$ & 200.0 & & & & & & & & & & 200.0 \\
\hline & & & Total & $4,990.8$ & $3,485.7$ & $2,616.8$ & & & & & & & & $11,093.3$ \\
\hline
\end{tabular}


SOLID WASTE PROGRAM

WBS 1.2.1

FY 1997 Program Plan

COST BASELINE BY YEAR BY ADS

Page 3 of 3

WHC-SP-1114, REV.2

(\$000s)

\begin{tabular}{|c|c|c|c|c|c|c|c|c|c|c|c|c|c|c|}
\hline RL WBS \# & ADS \# & TITLE & FUND TYPE & FY1997 & FY1998 & FY1999 & FY2000 & FY2001 & FY2002 & FY2003 & FY2004 & FY2005 & FY2006 & TOTAL \\
\hline \multirow[t]{4}{*}{1.2 .1 .4} & $2200-1$ & A7-Waste Storage & Line Item & $1,148.0$ & & & & & & & & & & $1,148.0$ \\
\hline & & s infrastructure & Total BCWS/PMB (1) & $1,148.0$ & & & & & & & & & & $1,148.0$ \\
\hline & & & Line Item Contingency (2) & 800.0 & & & & & & & & & & 800.0 \\
\hline & & & Total & $1,948.0$ & & & & & & & & & & $1,948.0$ \\
\hline \multirow[t]{8}{*}{ 1.2.1 } & All Direct & Solid Waste Program & Expense & $61,156.5$ & $69,240.6$ & 83.088 .3 & $90,007.1$ & $105,204.7$ & $128,877.1$ & $133,841.5$ & $128,491.3$ & $134,778.8$ & $132,593.0$ & $1,067,278.8$ \\
\hline & & & CENRTC & 565.2 & $2,222.2$ & $2,036.0$ & $3,696.2$ & $3,087.7$ & $4,032.0$ & $3,227.3$ & $3,314.5$ & $3,404.2$ & $4,970.4$ & 30.555 .7 \\
\hline & & & Line Item & $5,247.9$ & $2,700.0$ & 17.736 .2 & $17,394.8$ & $23,882.1$ & $26,927.7$ & $22,726.3$ & $27,945.7$ & $21,523.7$ & $22,104.5$ & $188,189.0$ \\
\hline & & & GPP & $3,072.5$ & $2,500.2$ & $3,457.5$ & $1,692.0$ & $1,579,4$ & $1,621.2$ & $1,663.0$ & $1,706.5$ & $1,751.3$ & $1,790.0$ & $20,833.7$ \\
\hline & & & $\begin{array}{l}\text { Total BCWS/PMB ( } 1 \text { ) } \\
\text { Mgmt Reserve (2) }\end{array}$ & $70,042.1$ & $76,663,0$ & $106,318.0$ & $112,790.0$ & $133,754.0$ & $161,458.0$ & $161,458.0$ & $161,458.0$ & $161,458.0$ & $161,458.0$ & $1,306,857.1$ \\
\hline & & & Line Item Contingency (2) & $1,000.0$ & & & & & & & & & & $1,000.0$ \\
\hline & & & Expected Carryover (3) & 450.0 & & & & & & & & & & 450.0 \\
\hline & & & Total & 71.492 .1 & $76,663.0$ & $106,318.0$ & $112,790.0$ & $133,754.0$ & $161,458.0$ & $161,458,0$ & $161,458.0$ & $161,458.0$ & $161,458.0$ & $1,308,307.1$ \\
\hline \multirow[t]{2}{*}{ 1.2.1.11 } & & Solid Waste Haz & Expense & 258.1 & & & & & & & & & & 258.1 \\
\hline & & Analysis Assessment & Total BCWSIPMB (1) & 258.1 & & & & & & & & & & 258.1 \\
\hline \multirow[t]{2}{*}{1.2 .1 .11} & & Solid Wasto $1 \mathrm{ML}$ & Expense & $6,775.9$ & & & & & & & & & & $6,775.9$ \\
\hline & & Pool Assessment & Total BCWS/PMB (1) & $6,775.9$ & & & & & & & & & & $6,775.9$ \\
\hline \multirow{2}{*}{\multicolumn{3}{|c|}{$\begin{array}{l}\text { PROGRAM BCWS/PMB } \\
\text { PROGRAM TOTAL }\end{array}$}} & & $77,076.2$ & $76,663.0$ & $106,318.0$ & $112,790.0$ & $133,754.0$ & $161,458.0$ & $161,458.0$ & $161,458.0$ & 161.458 .0 & $161,458.0$ & $1,313,891.2$ \\
\hline & & & & $78,526.2$ & $76,663.0$ & $106,318.0$ & $112,790.0$ & $133,754.0$ & $161,458.0$ & $161,458.0$ & $161,458.0$ & $161,458.0$ & $161,458.0$ & $1,315,341.2$ \\
\hline
\end{tabular}

(1) Budgeted Cost of Work Scheduled (BCWS) Equals Pertormance Measurement Baseline (PMB).

(2) Management Reserve And Line ltem Contingency Held By RL.

(3) Includes Expected Expense Carryover Requested By Formal Change Control in FY1997. 


\subsection{Basis of Estimate}

The Solid Waste Program, under direction from DOE-RL (ref. Letter No. 96-WPD-007), utilized the Liquid Effluent Programs (LEP) format and process for developing the Solid Waste Program Basis of Estimate (BOE) for Fiscal Year 1997 and outyears. This process used the activity-based cost (ABC) estimating methodology to create technically complete, fully documented, and defensible estimates. All of the BOEs for the Solid Waste Program used the process created by LEP as requested by DOERL.

The record copy of the Basis of Estimate for the Solid Waste Program resides with the appropriate Program Activity Engineer located in MO278/200 West Area. 
Exhibit: Program Average FTE Projections by COCS Categories

\section{Program WBS/Title: 1.2.1 SOLID WASTE PROGRAM Direct Funding ("A")}

\begin{tabular}{l|l}
\hline COCS & \multicolumn{1}{|c}{ Title } \\
\hline C000 & Crafts \\
C010 & Carpenters \\
C020 & Electricians \\
C030 & Heating Air-Conditioning and Refrig Mechanics (HVAC) \\
C040 & Machinists \\
C050 & Masons \\
C060 & Millwights \\
C070 & Painters \\
C080 & Plumbers and Pipefitters \\
C090 & Structural and Metal Workers \\
C100 & Vehicle and Mobile Equipment Mechanics \\
C110 & Welders \\
C120 & Other Crafts \\
E000 & Engineers \\
E010 & Chemical Engineers \\
E020 & Civil Engineers \\
E040 & Electrical Engineers \\
E050 & Environmental Engineers \\
E060 & Industrial Engineers \\
E070 & Mechanical Engineers \\
E080 & Nuclear Engineers \\
E090 & PetroleumMining Engineers \\
E100 & Plant Engineers \\
E110 & Quality Assurance/Control Engineers \\
E120 & Safety Engineers \\
E130 & Other Engineers \\
E140 & Construction Engineers \\
E150 & Project Engineers \\
G000 & General Admin, Secretarial \& Clerical Support \\
G010 & Administrative Assistants \\
G020 & Office Clerks (General) \\
G030 & Office Clerks (Specialized) \\
&
\end{tabular}

\begin{tabular}{|c|c|c|c|c|c|c|c|c|c|}
\hline FY 1997 & FY 1998 & FY 1999 & FY 2000 & FY 2001 & FY 2002 & FY 2003 & FY 2004 & FY 2005 & FY 2006 \\
\hline 38.6 & 39.8 & 38.6 & 37.3 & 41.3 & 44.8 & 45.0 & 44.9 & 39.0 & 42.9 \\
\hline 1.1 & 1.1 & 1.0 & 1.0 & 1.1 & 1.1 & 1.1 & 1.1 & 1.1 & 1.1 \\
\hline 12.5 & 12.9 & 12.9 & 12.4 & 13.1 & 13.0 & 13.1 & 13.0 & 13.0 & 13.0 \\
\hline 0.7 & 0.7 & 0.6 & 0.6 & 0.7 & 0.7 & 0.7 & 0.7 & 0.7 & 0.7 \\
\hline 0.02 & 0.0 & 0.0 & 0.0 & 0.0 & 0.0 & 0.0 & 0.0 & 0.0 & 0.0 \\
\hline 0.0 & 0.0 & 0.0 & 0.0 & 0.0 & 0.0 & 0.0 & 0.0 & 0.0 & 0.0 \\
\hline 7.8 & 8.4 & 8.1 & 7.9 & 8.0 & 8.0 & 8.0 & 8.0 & 8.0 & 7.9 \\
\hline 3.0 & 3.1 & 2.9 & 2.8 & 3.1 & 3.1 & 3.1 & 3.1 & 3.1 & 3.1 \\
\hline 3.0 & 3.2 & 3.1 & 3.0 & 3.0 & 2.9 & 2.9 & 2.9 & 2.9 & 2.9 \\
\hline 2.0 & 2.5 & 2.1 & 1.9 & 1.9 & 1.8 & 1.8 & 1.8 & 1.8 & 1.8 \\
\hline 0.9 & 1.1 & 1.1 & 1.1 & 3.1 & 7.1 & 7.1 & 7.1 & 1.2 & 5.1 \\
\hline 0.4 & 0.4 & 0.4 & 0.4 & 0.4 & 0.4 & 0.4 & 0.4 & 0.4 & 0.4 \\
\hline 7.2 & 6.7 & 6.4 & 6.1 & 6.8 & 6.8 & 6.8 & 6.8 & 6.8 & 6.8 \\
\hline 730.2 & 147.5 & 159.3 & 161.0 & 148.0 & 167.8 & 168.2 & 165.5 & 157.4 & 161.6 \\
\hline 9.9 & 12.0 & 12.2 & 12.3 & 12.5 & 12.7 & 12.7 & 12.7 & 12.7 & 12.7 \\
\hline 1.2 & 1.0 & 0.9 & 0.6 & 0.6 & 0.3 & 0.3 & 0.3 & 0.3 & 0.3 \\
\hline 7.6 & 6.6 & 7.3 & 6.7 & 6.9 & 7.7 & 7.7 & 7.7 & 7.7 & 7.7 \\
\hline 14.4 & 15.6 & 15.5 & 15.3 & 14.0 & 16.2 & 16.5 & 16.3 & 16.2 & 16.0 \\
\hline 0.1 & 0.1 & 0.1 & 0.1 & 0.1 & 0.2 & 0.2 & 0.2 & 0.2 & 0.2 \\
\hline 7.9 & 6.4 & 6.8 & 6.1 & 6.1 & 6.3 & 5.9 & 6.1 & 6.1 & 6.1 \\
\hline 3.2 & 2.7 & 2.6 & 3.1 & 3.4 & 3.3 & 3.3 & 3.3 & 3.3 & 3.3 \\
\hline 0.0 & 0.0 & 0.0 & 0.0 & 0.0 & 0.0 & 0.0 & 0.0 & 0.0 & 0.0 \\
\hline 29.5 & 32.8 & 33.6 & 35.5 & 30.0 & 32.7 & 32.9 & 32.0 & 35.5 & 35.5 \\
\hline 3.6 & 3.4 & 3.3 & 3.0 & 3.1 & 3.0 & 3.0 & 3.0 & 3.0 & 3.0 \\
\hline 11.9 & 12.4 & 14.7 & 14.2 & 12.5 & 18.7 & 19.4 & 20.3 & 12.8 & 14.4 \\
\hline 39.0 & 53.5 & 61.3 & 63.7 & 58.5 & 66.6 & 66.3 & 63.5 & 59.5 & 62.4 \\
\hline $\begin{array}{l}0.1 \\
1.8\end{array}$ & $\begin{array}{l}0.0 \\
0.8\end{array}$ & $\begin{array}{l}0.0 \\
0.8\end{array}$ & $\begin{array}{l}0.0 \\
0.3\end{array}$ & $\begin{array}{l}0.0 \\
0.2\end{array}$ & $\begin{array}{l}0.0 \\
0.1\end{array}$ & 0.0 & $\begin{array}{l}0.0 \\
0.1\end{array}$ & 0.0 & 0.0 \\
\hline 30.2 & 29.8 & 28.3 & 28.4 & 27.9 & 27.9 & 28.7 & 27.5 & 27.9 & 27.9 \\
\hline 1.1 & 1.1 & 0.8 & 0.8 & 0.8 & 0.8 & 0.8 & 0.8 & 0.8 & 0.8 \\
\hline 5.8 & 5.9 & 5.5 & 5.9 & 6.0 & 6.0 & 6.8 & 5.1 & 6.0 & 6.0 \\
\hline 7.4 & 6.9 & 6.6 & 6.1 & 6.5 & 6.5 & 6.5 & 6.5 & 6.5 & 6.5 \\
\hline
\end{tabular}


Exhibit: Program Average FTE Projections by COCS Categories

Program WBS/Title: 1.2.1 SOLID WASTE PROGRAM Direct Funding ("A")

\begin{tabular}{|c|c|c|c|c|c|c|c|c|c|c|c|}
\hline $\operatorname{cocs}$ & Title & FY 1997 & FY 1998 & FY 1999 & FY 2000 & FY 2001 & FY 2002 & FY 2003 & FY 2004 & FY 2005 & FY 2006 \\
\hline G040 & Secretaries & 15.9 & 15.9 & 15.3 & 15.5 & 14.6 & 14.7 & 14.6 & 15.1 & 14.6 & 14.6 \\
\hline G050 & Typist and Word Processors & .0 & .0 & .0 & .0 & .0 & .0 & .0 & .0 & .0 & .0 \\
\hline G060 & Other General Admin, Secretarial and Clerical Support & .0 & .0 & .0 & .0 & .0 & .0 & .0 & .0 & .0 & .0 \\
\hline LOOO & Laborers and General Service Workers & 10.1 & 12.5 & 11.1 & 11.0 & 71.2 & 11.1 & 11.0 & 11.0 & 11.0 & 11.0 \\
\hline L010 & Firefighters & .0 & .0 & .0 & .0 & .0 & .0 & .0 & .0 & .0 & .0 \\
\hline L020 & Food Service Workers & .0 & .0 & .0 & .0 & .0 & .0 & .0 & .0 & .0 & .0 \\
\hline L030 & Janitors and Cleaners & 6.7 & 6.7 & 6.7 & 6.7 & 6.7 & 6.7 & 6.7 & 6.7 & 6.7 & 6.7 \\
\hline L040 & Laundry Workers & .0 & .0 & .0 & .0 & .0 & .0 & .0 & .0 & .0 & .0 \\
\hline L.050 & Handlers, Helpers and Laborers (General) & 0.2 & 0.1 & 0.3 & 0.3 & 0.3 & 0.3 & 0.3 & 0.3 & 0.3 & 0.3 \\
\hline L060 & Handlers, Helpers and Laborers (Specialized) & 0.2 & 0.2 & 0.2 & 0.2 & 0.2 & 0.2 & 0.2 & 0.2 & 0.2 & 0.2 \\
\hline L070 & Light Vehicle Drivers & 2.9 & 5.5 & 3.8 & 3.7 & 3.9 & 3.8 & 3.8 & 3.8 & 3.8 & 3.8 \\
\hline L.080 & Security Guards & .0 & .0 & .0 & .0 & .0 & .0 & .0 & .0 & .0 & .0 \\
\hline LO90 & Other Laborers and General Services Workers & .0 & .0 & .0 & .0 & .0 & .0 & .0 & .0 & 0 & .0 \\
\hline M000 & Gen Mgrs, Exec, 1st Line Suprv'sr \& Prog/Proj Mgrs & 46.0 & 50.9 & 50.8 & 49.9 & 46.2 & 48.4 & 48.4 & 48.4 & 48.4 & 48.4 \\
\hline Mo10 & First Line Supervisors & 17.4 & 22.9 & 23.8 & 23.7 & 20.1 & 22.3 & 22.3 & 22.3 & 22.3 & 22.3 \\
\hline M020 & General Managers and Executives & 25.1 & 24.7 & 24.4 & 24.5 & 24.4 & 24.4 & 24.4 & 24.4 & 24.4 & 24.4 \\
\hline M030 & Project and Program Managers & 3.4 & 3.1 & 2.1 & 1.1 & 1.1 & 1.1 & 1.1 & 1.1 & 1.1 & 1.1 \\
\hline M040 & Other Managers & 0.1 & 0.2 & 0.5 & 0.5 & 0.5 & 0.5 & 0.5 & 0.5 & 0.5 & 0.5 \\
\hline P000 & Professional Administrative \& Related Occupations & 59.7 & 61.0 & 60.6 & 60.3 & 62.6 & 63.3 & 63.6 & 63.3 & 63.8 & 62.6 \\
\hline P010 & Accountants and Auditors & 10.6 & 13.0 & 13.0 & 12.5 & 11.4 & 11.3 & 12.1 & 12.0 & 12.0 & $\$ 1.1$ \\
\hline $\mathrm{P} 020$ & Architects & 0.0 & 0.0 & 0.0 & 0.0 & 0.0 & 0.0 & 0.0 & 0.0 & 0.0 & 0.0 \\
\hline P030 & Buyers, Procurement and Contracting Specialists & 0.12 & 0.1 & 0.1 & 0.1 & 0.1 & 0.2 & 0.2 & 0.2 & 0.2 & 0.2 \\
\hline P040 & Communications Specialists & 0.0 & 0.0 & 0.0 & 0.0 & 0.0 & 0.0 & 0.0 & 0.0 & 0.0 & 0.0 \\
\hline P050 & Compliance Inspectors & 0.8 & 0.8 & 0.8 & 0.8 & 0.8 & 0.8 & 0.8 & 0.8 & 0.8 & 0.8 \\
\hline P060 & Computer Systems Analysts & 2.4 & 2.3 & 2.4 & 2.4 & 2.4 & 2.7 & 2.7 & 2.7 & 2.7 & 2.7 \\
\hline P070 & Cost Estimators and Planners and Schedulers & 16.5 & 16.7 & 16.4 & 16.5 & 18.0 & 18.0 & 18.1 & 18.1 & 18.1 & 18.0 \\
\hline P080 & Health Physicists & 7.5 & 6.7 & 7.1 & 6.8 & 7.8 & 8.1 & 7.7 & 7.4 & 7.9 & 7.9 \\
\hline P090 & Industrial Hygienists & 2.6 & 2.6 & 2.4 & 2.4 & 2.6 & 2.6 & 2.6 & 2.6 & 2.6 & 2.6 \\
\hline P100 & Lawyers & 0.0 & 0.1 & 0.0 & 0.0 & 0.0 & 0.0 & 0.0 & 0.0 & 0.0 & 0.0 \\
\hline P110 & Personnel and Labor Relations Specialists & 0.0 & 0.0 & 0.0 & 0.0 & 0.0 & 0.0 & 0.0 & 0.0 & 0.0 & 0.0 \\
\hline P120 & Physicians & 0.0 & 0.0 & 0.0 & 0.0 & 0.0 & 0.0 & 0.0 & 0.0 & 0.0 & 0.0 \\
\hline P130 & Physician Assist, Nurses \& Oth Medical Supt Occup'tns & 0.0 & 0.0 & 0.0 & 0.0 & 0.0 & 0.0 & 0.0 & 0.0 & 0.0 & 0.0 \\
\hline
\end{tabular}


WBS 1.2.1

\section{Exlibit: Program Average FTE Projections by CoCs Categories}

\section{Program WBS/Title: 1.2.1 SOLID WASTE PROGRAM Direct Funding ("A")}

\begin{tabular}{|c|c|c|c|c|c|c|c|c|c|c|c|}
\hline COCS & Title & FY 1997 & FY 1998 & FY 1999 & FY 2000 & FY 2001 & FY 2002 & FY 2003 & FY 2004 & FY 2005 & FY 2006 \\
\hline P440 & Safeguards and Other Security Specialists & 0.4 & 0.9 & 1.1 & 1.0 & 1.2 & 1.1 & 1.1 & 1.1 & 1.1 & 1.1 \\
\hline P150 & Trainers & 8.1 & 7.9 & 8.1 & 8.1 & 8.2 & 8.2 & 8.2 & 8.2 & 8.2 & 7.9 \\
\hline $\begin{array}{l}\text { P160 } \\
\text { P170 }\end{array}$ & $\begin{array}{l}\text { Technical Writers, and Editors } \\
\text { Other Administrative \& Professional Other Occupations }\end{array}$ & $\begin{array}{l}1.6 \\
9.1\end{array}$ & $\begin{array}{l}1.3 \\
8.6\end{array}$ & $\begin{array}{l}1.2 \\
8.0\end{array}$ & $\begin{array}{l}1.2 \\
8.5\end{array}$ & $\begin{array}{l}1.2 \\
9.1\end{array}$ & $\begin{array}{l}1.2 \\
9.1\end{array}$ & $\begin{array}{l}1.2 \\
9.1\end{array}$ & $\begin{array}{l}1.2 \\
9.1\end{array}$ & $\begin{array}{l}1.2 \\
9.1\end{array}$ & $\begin{array}{l}1.2 \\
9.1\end{array}$ \\
\hline $\mathbf{R} 000$ & Operators & 63.1 & 78.1 & 85.9 & 93.3 & 92.9 & 127.8 & 127.8 & 127.8 & 98.1 & 109.9 \\
\hline R010 & Chemical System Operators & 0.0 & 0.0 & 0.0 & 0.0 & 0.0 & 0.0 & 0.0 & 0.0 & 0.0 & 0.0 \\
\hline R020 & Drillers & 0.0 & 0.0 & 0.0 & 0.0 & 0.0 & 0.0 & 0.0 & 0.0 & 0.0 & 0.0 \\
\hline R030 & Material Moving Equipment Operators & 3.2 & 4.4 & 2.9 & 5.1 & 1.2 & 1.1 & 1.1 & 1.1 & 3.1 & 3.1 \\
\hline R040 & Nuclear Plant Operators & 2.0 & 1.6 & 1.6 & 1.5 & 1.5 & 1.0 & 1.0 & 1.0 & 1.0 & 1.0 \\
\hline R050 & Nuclear Waste Process Operators & 57.9 & 71.9 & 79.8 & 82.7 & 84.3 & 107.8 & 107.8 & 107.8 & 92.0 & 97.8 \\
\hline R060 & Production Systems Operators & 0.0 & 0.0 & 0.0 & 0.0 & 0.0 & 0.0 & 0.0 & 0.0 & 0.0 & 0.0 \\
\hline R070 & Utilities Operators & 0.0 & 0.2 & 0.0 & 0.0 & 0.0 & 0.0 & 0.0 & 0.0 & 0.0 & 0.0 \\
\hline R080 & Other Operators & 0.0 & 0.0 & 1.5 & 4.0 & 5.9 & 17.8 & 17.8 & 17.8 & 2.0 & 7.9 \\
\hline Sooo & Scientists & 74.9 & 10.1 & 9.5 & 10.4 & 10.4 & 70.6 & 10.6 & 10.6 & 10.6 & 10.6 \\
\hline S010 & Chemists & 2.0 & 2.3 & 2.3 & 1.5 & 1.5 & 1.5 & 1.5 & 1.5 & 1.5 & 1.5 \\
\hline S020 & Environmental Scientists & 5.5 & 4.3 & 3.5 & 3.2 & 3.2 & 3.1 & 3.1 & 3.1 & 3.1 & 3.1 \\
\hline S030 & Geologists & 1.0 & 0.3 & 0.3 & 0.3 & 0.3 & 0.3 & 0.3 & 0.3 & 0.3 & 0.3 \\
\hline S040 & Life Scientists & 1.0 & 1.3 & 1.3 & 1.3 & 1.3 & 1.4 & 1.4 & 1.4 & 1.4 & 1.4 \\
\hline S050 & Materials Scientists & 0.0 & 0.0 & 0.0 & 0.0 & 0.0 & 0.0 & 0.0 & 0.0 & 0.0 & 0.0 \\
\hline s060 & Mathematicians & 0.0 & 0.0 & 0.0 & 0.0 & 0.0 & 0.0 & 0.0 & 0.0 & 0.0 & 0.0 \\
\hline S070 & Physicists & 0.0 & 0.0 & 0.0 & 0.0 & 0.0 & 0.0 & 0.0 & 0.0 & 0.0 & 0.0 \\
\hline S080 & Social Scientists & 0.0 & 0.0 & 0.0 & 0.0 & 0.0 & 0.0 & 0.0 & 0.0 & 0.0 & 0.0 \\
\hline sogo & Other Scientists & 0.9 & 0.0 & 0.0 & 0.0 & 0.0 & 0.0 & 0.0 & 0.0 & 0.0 & 0.0 \\
\hline S100 & Computer Scientists & 4.5 & 2.0 & 2.1 & 4.0 & 4.1 & 4.3 & 4.3 & 4.3 & 4.3 & 4.3 \\
\hline TOOO & Technicians & 54.2 & 61.9 & 64.9 & 64.5 & 61.7 & 76.2 & 76.8 & 77.7 & 72.8 & 75.7 \\
\hline T010 & Computer Operator/Coders & 0.8 & 0.5 & 0.5 & 0.5 & 0.5 & 0.6 & 0.6 & 0.6 & 0.6 & 0.6 \\
\hline T020 & Drafters & 7.9 & 7.2 & 5.9 & 6.0 & 6.8 & 6.8 & 6.8 & 6.8 & 6.8 & 6.8 \\
\hline T030 & Engineering Technicians & 1.0 & 1.0 & 1.1 & 1.1 & 1.1 & 1.3 & 1.3 & 1.3 & 1.3 & 1.3 \\
\hline T040 & Environmental Sciences Technicians & 0.0 & 0.0 & 0.0 & 0.0 & 0.0 & 0.0 & 0.0 & 0.0 & 0.0 & 0.0 \\
\hline T050 . & Health Physics Technicians & 34.7 & 41.5 & 44.6 & 44.9 & 42.4 & 54.5 & 55.0 & 55.9 & 51.0 & 53.9 \\
\hline
\end{tabular}


Exhibit: Program Average FTE Projections by COCS Categories Program WBS/Title: 1.2.1 SOLID WASTE PROGRAM Direct Funding ("A")

\begin{tabular}{|c|c|c|c|c|c|c|c|c|c|c|c|}
\hline COCs & Titie & FY 1997 & FY 1998 & FY 1999 & FY 2000 & FY 2001 & FY 2002 & FY 2003 & FY 2004 & FY 2005 & FY 2006 \\
\hline T060 & Industrial Safety and Health Technicians & 0.6 & 0.8 & 1.7 & 1.7 & 0.1 & 2.3 & 2.3 & 2.3 & 2.3 & 2.3 \\
\hline T070 & Instrument and Control Technicians & 8.2 & 8.4 & 8.3 & 7.8 & 8.1 & 8.0 & 8.1 & 8.0 & 8.0 & 8.0 \\
\hline T080 & Laboratory Techinicians & 0.0 & 0.0 & 0.0 & 0.0 & 0.0 & 0.0 & 0.0 & 0.0 & 0.0 & 0.0 \\
\hline T090 & Media Technicians & 0.0 & 0.0 & 0.0 & 0.0 & 0.0 & 0.0 & 0.0 & 0.0 & 0.0 & 0.0 \\
\hline T100 & Survey and Mapping & 0.0 & 0.0 & 0.0 & 0.0 & 0.0 & 0.0 & 0.0 & 0.0 & 0.0 & 0.0 \\
\hline $\mathrm{T} 110$ & Other Technicians & 0.9 & 2.6 & 2.8 & 2.5 & 2.8 & 2.8 & 2.8 & 2.8 & 2.8 & 2.8 \\
\hline Total & & 447.1 & 492.5 & 509.9 & 516.0 & 502.2 & 577.9 & 580.2 & 576.9 & 529.0 & 550.5 \\
\hline
\end{tabular}


Exhibit: Program Average FTE Projections by COCS Categories Program WBS/Title: 1.2.1 SOLID WASTE PROGRAM Indirect Funding ("ML")

\begin{tabular}{|c|c|c|c|c|c|c|c|c|c|c|c|}
\hline Cocs & Title & FY 1997 & FY 1998 & FY 1999 & FY 2000 & FY 2001 & FY 2002 & FY 2003 & FY 2004 & FY 2005 & FY 2006 \\
\hline $\mathbf{C 0 0 0}$ & Crafts & & & & & & & & & & \\
\hline 0010 & Carpenters & & & & & & & & & & \\
\hline $\mathrm{C} 020$ & Electricians & & & & & & & & & & \\
\hline $\mathrm{C} 030$ & Heating Air-Conditioning and Refrig Mechanics (HVAC) & & & & & & & & & & \\
\hline $\mathrm{CO40}$ & Machinists & & & & & & & & & & \\
\hline $\cos 0$ & Masons & & & & & & & & & & \\
\hline $\mathrm{c060}$ & Millwrights & & & & & & & & & & \\
\hline $\mathrm{C} 070$ & Painters & & & & & & & & & & \\
\hline C080 & Plumbers and Pipefitters & & & & & & & & & & \\
\hline $\operatorname{cog}$ & Structural and Metal Workers & & & & & & & & & & \\
\hline C100 & Vehicle and Mobile Equipment Mechanics & & & & & & & & & & \\
\hline C110 & Welders & & & & & & & & & & \\
\hline $\mathrm{C} 120$ & Other Crafts & & & & & & & & & & \\
\hline$E 000$ & Engineers & 17.9 & & & & & & L & & & \\
\hline E010 & Chemical Engineers & 0.04 & & & & & & & & & \\
\hline E020 & $\begin{array}{l}\text { Civil Engineers } \\
\text { S }\end{array}$ & 0.03 & & & & & & & & & \\
\hline E040 & Electrical Engineers & & & & & & & & & & \\
\hline E050 & Environmental Engineers & 10.3 & & & & & & & & & \\
\hline E060 & Industrial Engineers & & & & & & & & & & \\
\hline E070 & Mechanical Engineers & 0.02 & & & & & & & & & \\
\hline E080 & Nuclear Engineers & 0.04 & & & & & & & & & \\
\hline E090 & Petroleum/Mining Engineers & & & & & & & & & & \\
\hline E100 & Plant Engineers & 2.85 & & & & & & & & & \\
\hline E110 & Quality Assurance/Control Engineers & 0.23 & & & & & & & & & \\
\hline$E 120$ & Safety Engineers & 0.03 & & & & & & & & & \\
\hline E130 & Other Engineers & 4.17 & & & & & & & & & \\
\hline E140 & Construction Engineers & 0.22 & & & & & & & & & \\
\hline
\end{tabular}


Exhibit: Program Average FTE Projections by COCS Categories

Program WBS/Title: 1.2.1 SOLID WASTE PROGRAM Indirect Funding ("ML")

\begin{tabular}{|c|c|c|c|c|c|c|c|c|c|c|c|}
\hline $\mathrm{cocs}$ & Title & FY 1997 & FY 1998 & FY 1999 & FY 2000 & FY 2001 & FY 2002 & FY 2003 & FY 2004 & FY 2005 & FY 2006 \\
\hline G000 & General Admin, Secretarial \& Clerical Support & 0.6 & & & & & & & & & \\
\hline G010 & Administrative Assistants & & & & & & & & & & \\
\hline G020 & Office Clerks (General) & 0.5 & & & & & & & & & \\
\hline G030 & Office Clerks (Specialized) & & & & & & & & & & \\
\hline G040 & Secretaries & 0.1 & & & & & & & & & \\
\hline G050 & Typist and Word Processors & & & & & & & & & & \\
\hline Lo00 & $\begin{array}{l}\text { Other General Admin, Secretarial and Clerical Support } \\
\text { Laborers and General Service Workers }\end{array}$ & $\overline{1.4}$ & & & & & & & & & \\
\hline L010 & Firefighters & & & & & & & & & & \\
\hline L020 & Food Service Workers & & & & & & & & & & \\
\hline L030 & Janitors and Cleaners & & & & & & & & & & \\
\hline L040 & Laundry Workers & & & & & & & & & & \\
\hline L050 & Handlers, Helpers and Laborers (General) & & & & & & & & & & \\
\hline L060 & Handlers, Helpers and Laborers (Specialized) & & & & & & & & & & \\
\hline L070 & Light Vehicle Drivers & 1.4 & & & & & & & & & \\
\hline $\begin{array}{l}\text { LO80 } \\
\text { L090 }\end{array}$ & $\begin{array}{l}\text { Security Guards } \\
\text { Other Laborers and General Services Workers }\end{array}$ & & & & & & & & & & \\
\hline Mo00 & Gen Mgrs, Exec, 1st Line Suprv'sr \& Prog/Proj Mgrs & .3 & & & & & & & & & \\
\hline M010 & First Line Supervisors & 0.1 & & & & & & & & & \\
\hline M020 & General Managers and Executives & & & & & & & & & & \\
\hline M030 & Project and Program Managers & 0.2 & & & & & & & & & \\
\hline M040 & Other Managers & & & & & & & & & & \\
\hline
\end{tabular}




\section{Exhibit: Program Average FTE Projections by COCS Categories}

\section{Program WBS/Title: 1.2.1 SOLID WASTE PROGRAM Indirect Funding ("ML")}

\begin{tabular}{|c|c|c|c|c|c|c|c|c|c|c|c|}
\hline cocs & Title & FY 1997 & FY 1998 & FY 1999 & FY2000 & FY 2001 & FY 2002 & FY 2003 & FY 2004 & FY 2005 & FY 2006 \\
\hline P000 & Professional Administrative \& Related Occupations & 7.9 & & & & & & & & & \\
\hline P010 & Accountants and Auditors & $\overline{0.10}$ & & & & & & & & & \\
\hline P020 & Architects & & & & & & & & & & \\
\hline P030 & Buyers, Procurement and Contracting Specialists & 0.10 & & & & & & & & & \\
\hline P040 & Communications Specialists & & & & & & & & & & \\
\hline P050 & Compliance Inspectors & 0.20 & & & & & & & & & \\
\hline P060 & Computer Systems Analysts & & & & & & & & & & \\
\hline P070 & Cost Estimators and Planners and Schedulers & 1.45 & & & & & & & & & \\
\hline P080 & Health Physicists & 0.33 & & & & & & & & & \\
\hline P090 & Industrial Hygienists & 0.65 & & & & & & & & & \\
\hline P1:10 & Lawyers & & & & & & & & & & \\
\hline P110 & Personnel and Labor Relations Specialists & & & & & & & & & & \\
\hline P120 & Physicians & & & & & & & & & & \\
\hline P130 & Physician Assist, Nurses \& Oth Medical Supt Occup'tns & & & & & & & & & & \\
\hline P140 & Safeguards and Other Security Specialists & 3.06 & & & & & & & & & \\
\hline P150 & Trainers & & & & & & & & & & \\
\hline $\begin{array}{l}\text { P160 } \\
\text { P170 }\end{array}$ & $\begin{array}{l}\text { Technical Writers, and Editors } \\
\text { Other Administrative \& Professional Other Occupations }\end{array}$ & 1.97 & & & & & & & & & \\
\hline $\mathbf{R} 000$ & Operators & 12.5 & & & & & & & & & \\
\hline
\end{tabular}

R010 Chemical System Operators

R020 Drillers

R030 Material Moving Equipment Operators

R040 Nuclear Plant Operators

R050 Nuclear Waste Process Operators

R060 Production Systems Operators

R070 Utilities Operators

R080 Other Operators 
Exhibit: Program Average FTE Projections by COCS Categories Program WBS/Title: 1.2.1 SOLID WASTE PROGRAM Indirect Funding ("ML")

\begin{tabular}{|c|c|c|c|c|c|c|c|c|c|c|c|}
\hline COCS & Title & FY 1997 & FY 1998 & FY 1999 & FY 2000 & FY 2001 & FY 2002 & FY 2003 & FY 2004 & FY 2005 & FY 2006 \\
\hline 5000 & Scientists & 3.7 & & & & & & & & & \\
\hline S010 & Chemists & & & & & & & & & & \\
\hline s020 & Environmental Scientists & 0.81 & & & & & & & & & \\
\hline S030 & Geologists & & & & & & & & & & \\
\hline S040 & Life Scientists & 0.85 & & & & & & & & & \\
\hline s050 & Materials Scientists & & & & & & & & & & \\
\hline S060 & Mathematicians & & & & & & & & & & \\
\hline S070 & Physicists & & & & & & & & & & \\
\hline S080 & Social Scientists & & & & & & & & & & \\
\hline so90 & Other Scientists & & & & & & & & & & \\
\hline S100 & Computer Scientists & 2.01 & & & & & & & & & \\
\hline Tooo & Technicians & 10.7 & & & & & & & & & \\
\hline T010 & Computer Operator/Coders & 1.11 & & & & & & & & & \\
\hline T020 & Drafters & & & & & & & & & & \\
\hline T030 & Engineening Technicians & 2.98 & & & & & & & & & \\
\hline T040 & Environmental Sciences Technicians & & & & & & & & & & \\
\hline T050 & Health Physics Technicians & 5.69 & & & & & & & & & \\
\hline T060 & Industrial Safety and Health Technicians & & & & & & & & & & \\
\hline T070 & Instrument and Control Technicians & & & & & & & & & & \\
\hline To80 & Laboratory Technicians & & & & & & & & & & \\
\hline T090 & Media Technicians & & & & & & & & & & \\
\hline T100 & Survey and Mapping & & & & & & & & & & \\
\hline T110 & Other Technicians & 0.90 & & & & & & & & & \\
\hline Total & & 55 & .0 & .0 & .0 & .0 & .0 & .0 & .0 & .0 & .0 \\
\hline
\end{tabular}


Exhibit: Program Average FTE Projections by COCS Categories

Program WBS/Title: 1.2.1 SOLID WASTE PROGRAM Indirect Funding ("MD")

\begin{tabular}{|c|c|c|c|c|c|c|c|c|c|c|c|}
\hline $\operatorname{cocs}$ & Title & FY 1997 & FY 1998 & FY 1999 & FY 2000 & FY 2001 & FY 2002 & FY 2003 & FY 2004 & FY 2005 & FY 2006 \\
\hline $\mathbf{C 0 0 0}$ & Crafts & & & & & & & & & & \\
\hline $\mathrm{C010}$ & Carpenters & & & & & & & & & & \\
\hline $\mathrm{C} 020$ & Electricians & & & & & & & & & & \\
\hline $\cos 0$ & Heating Air-Conditioning and Refrig Mechanics (HVAC) & & & & & & & & & & \\
\hline $\mathrm{CO40}$ & Machinists & & & & & & & & & & \\
\hline $\cos 0$ & Masons & & & & & & & & & & \\
\hline $\mathrm{c060}$ & Millwrights & & & & & & & & & & \\
\hline $\mathrm{CO70}$ & Painters & & & & & & & & & & \\
\hline $\mathrm{co80}$ & Plumbers and Pipefitters & & & & & & & & & & \\
\hline C090 & Structural and Metal Workers & & & & & & & & & & \\
\hline C100 & Vehicle and Mobile Equipment Mechanics & & & & & & & & & & \\
\hline $\begin{array}{l}\mathrm{C} 110 \\
\mathrm{C} 120\end{array}$ & $\begin{array}{l}\text { Welders } \\
\text { Other Crafts }\end{array}$ & & & & & & & & & & \\
\hline EOOO & $\begin{array}{l}\text { Other Crafts } \\
\text { Engineers }\end{array}$ & 1 & & 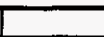 & & & 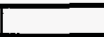 & & & L & I \\
\hline E010 & Chemical Engineers & 0.6 & & & & & & & & & \\
\hline E020 & Civil Engineers & & & & & & & & & & \\
\hline E040 & Electrical Engineers & & & & & & & & & & \\
\hline EO:O0 & Environmental Engineers & & & & & & & & & & \\
\hline E060 & Industrial Engineers & & & & & & & & & & \\
\hline E070 & Mechanical Engineers & & & & & & & & & & \\
\hline EOBO & Nuclear Engineers & & & & & & & & & & \\
\hline E090 & Petroleum/Mining Engineers & & & & & & & & & & \\
\hline E100 & Plant Engineers & & & & & & & & & & \\
\hline E110 & Quality Assurance/Control Engineers & & & & & & & & & & \\
\hline E120 & Safety Engineers & & & & & & & & & & \\
\hline $\begin{array}{l}E 130 \\
\text { E140 }\end{array}$ & $\begin{array}{l}\text { Other Engineers } \\
\text { Construction Engineers }\end{array}$ & & & & & & & & & & \\
\hline
\end{tabular}


Exhibit: Program Average FTE Projections by COCS Categories

Program WBS/Title: 1.2.1 SOLID WASTE PROGRAM Indirect Funding ("MD")

\begin{tabular}{|c|c|c|c|c|c|c|c|c|c|c|c|}
\hline COCS & Title & FY 1997 & FY 1998 & FY 1999 & FY 2000 & FY 2001 & FY 2002 & FY 2003 & FY 2004 & FY 2005 & FY 2006 \\
\hline G000 & General Admin, Secretarial \& Clerical Support & & & & & & & & & & \\
\hline G010 & Administrative Assistants & & & & & & & & & & \\
\hline G020 & Office Clerks (General) & & & & & & & & & & \\
\hline G030 & Office Clerks (Specialized) & & & & & & & & & & \\
\hline G040 & Secretaries & & & & & & & & & & \\
\hline $\begin{array}{l}\text { G050 } \\
\text { G060 }\end{array}$ & $\begin{array}{l}\text { Typist and Word Processors } \\
\text { Other General Admin Secretarial and Clerical Support }\end{array}$ & & & & & & & & & & \\
\hline Looo & Laborers and General Service Workers & & & & & & & & & & \\
\hline L010 & Firefighters & & & & & & & & & & \\
\hline L020 & Food Service Workers & & & & & & & & & & \\
\hline L030 & Janitors and Cleaners & & & & & & & & & & \\
\hline L040 & Laundry Workers & & & & & & & & & & \\
\hline L050 & Handlers, Helpers and Laborers (General) & & & & & & & & & & \\
\hline Lo60 & Handlers, Helpers and Laborers (Specialized) & & & & & & & & & & \\
\hline L070 & Light Vehicle Drivers & & & & & & & & & & \\
\hline LO80 & Security Guards & & & & & & & & & & \\
\hline $\begin{array}{l}\text { Lo90 } \\
\text { M000 }\end{array}$ & $\begin{array}{l}\text { Other Laborers and General Services Workers } \\
\text { Gen Mgrs, Exec, 1st Line Suprv'sr \& Prog/Proj Mgrs }\end{array}$ & & & & & & & & & & \\
\hline
\end{tabular}

M010 First Line Supervisors

M0?0 General Managers and Executives

M030 Project and Program Managers

M040 Other Managers 
Exhibit: Program Average FTE Projections by COCS Categories

Program WBS/Title: 1.2.1 SOLID WASTE PROGRAM Indirect Funding ("MD")

\begin{tabular}{|c|c|c|c|c|c|c|c|c|c|c|c|}
\hline COCS & \begin{tabular}{|c|} 
\\
Title \\
\end{tabular} & FY 1997 & FY 1998 & FY 1999 & FY 2000 & FY 2001 & FY 2002 & FY 2003 & FY 2004 & FY 2005 & FY 2006 \\
\hline POOO & Professional Administrative \& Related Occupations & 2 & & & & & & & & & \\
\hline P010 & Accountants and Auditors & & & & & & & & & & \\
\hline P020 & Architects & & & & & & & & & & \\
\hline P030 & Buyers, Procurement and Contracting Specialists & & & & & & & & & & \\
\hline P040 & Communications Specialists & & & & & & & & & & \\
\hline P050 & Compliance Inspectors & & & & & & & & & & \\
\hline P060 & Computer Systems Analysts & & & & & & & & & & \\
\hline P070 & Cost Estimators and Planners and Schedulers & & & & & & & & & & \\
\hline P080 & Health Physicists & & & & & & & & & & \\
\hline P090 & Industrial Hygienists & & & & & & & & & & \\
\hline$P 100$ & Lawyers & & & & & & & & & & \\
\hline $\mathrm{P} 110$ & Personnel and Labor Relations Specialists & & & & & & & & & & \\
\hline $\mathbf{P} 120$ & Physicians & & & & & & & & & & \\
\hline P130 & Physician Assist, Nurses \& Oth Medical Supt Occup'tns & & & & & & & & & & \\
\hline P140 & Safeguards and Other Security Specialists & 1.7 & & & & & & & & & \\
\hline P150 & Trainers & & & & & & & & & & \\
\hline $\begin{array}{l}\text { P160 } \\
\text { P170 }\end{array}$ & $\begin{array}{l}\text { Technical Writers, and Editors } \\
\text { Other Administrative \& Professional Other Occupations }\end{array}$ & & & & & & & & & & \\
\hline $\mathbf{R} 000$ & Operators & & & & & & & & & & \\
\hline
\end{tabular}

R010 Chemical System Operators

R020 Drillers

R030 Material Moving Equipment Operators

R040 Nuclear Plant Operators

R050 Nuclear Waste Process Operators

R030 Production Systems Operators

R070 Utilities Operators

R080 Other Operators 


\section{Exhibit: Program Average FTE Projections by COCS Categories}

Program WBS/Title: 1.2.1 SOLID WASTE PROGRAM Indirect Funding ("MD")

\begin{tabular}{|c|c|c|c|c|c|c|c|c|c|c|c|}
\hline COCS & Title & FY 1997 & FY 1998 & FY 1999 & FY 2000 & FY 2001 & FY 2002 & FY 2003 & FY 2004 & FY 2005 & FY 2006 \\
\hline 5000 & Scientists & 1 & & & & & & & & & \\
\hline S010 & Chemists & 0.1 & & & & & & & & & \\
\hline S020 & Environmental Scientists & 0.4 & & & & & & & & & \\
\hline S030 & Geologists & & & & & & & & & & \\
\hline S040 & Life Scientists & & & & & & & & & & \\
\hline s050 & Materials Scientists & & & & & & & & & & \\
\hline S060 & Mathematicians & & & & & & & & & . & \\
\hline S070 & Physicists & & & & & & & & & & \\
\hline S080 & Social Scientists & & & & & & & & & & \\
\hline so90 & Other Scientists & & & & & & & & & & \\
\hline S100 & Computer Scientists & & & & & & & & & & \\
\hline TO00 & Technicians & & & & & & & & & & \\
\hline T010 & Computer Operator/Coders & & & & & & & & & & \\
\hline T020 & Drafters & & & & & & & & & & \\
\hline T030 & Engineering Technicians & & & & & & & & & & \\
\hline T040 & Environmental Sciences Technicians & & & & & & & & & & \\
\hline T050 & Health Physics Technicians & & & & & & & & & & \\
\hline T060 & Industrial Safety and Health Technicians & & & & & & & & & & \\
\hline T070 & Instrument and Control Technicians & & & & & & & & & & \\
\hline T080 & Laboratory Technicians & & & & & & & & & & \\
\hline T090 & Media Technicians & & & & & & & & & & \\
\hline $\begin{array}{l}T 100 \\
T 110\end{array}$ & $\begin{array}{l}\text { Survey and Mapping } \\
\text { Other Technicians }\end{array}$ & & & & & & & & & & \\
\hline Total & & 2.8 & & & & & & & & & \\
\hline
\end{tabular}




\begin{tabular}{|c|c|c|c|}
\hline \multicolumn{4}{|c|}{$\begin{array}{c}\text { PROJECT WORK SCOPE MAPPING } \\
- \text { FY } 1996 \text { to FY } 1997-\end{array}$} \\
\hline \multirow{2}{*}{ 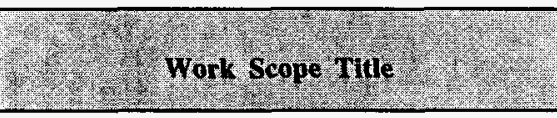 } & \multicolumn{2}{|c|}{$(1)$ Identliters } & 3) \\
\hline & FY 1996 & FY 1907 & (1) comment \\
\hline Product Line Management and Training & $\begin{array}{l}\text { Included in most } \\
\text { direct activities }\end{array}$ & $\begin{array}{l}\text { IA1M01, 1A1M04, } \\
\text { 1A1M02, 1A1M03, } \\
\text { 1A3M01, }\end{array}$ & $\begin{array}{l}\text { Management accounts previously spread across } \\
\text { all cost accounts in FY } 96 \text {. Consolidated in } \\
\text { FY97 for activity management and ABC } \\
\text { methodology. }\end{array}$ \\
\hline sWITS & 1A1901 & 1A1A07 & $\begin{array}{l}\text { Workscope removed from the Systems Eng. } \\
\text { cost account to a new cost account titled Data } \\
\text { Management. }\end{array}$ \\
\hline Safety and Health & overhead & 1A1A08 & Safety and Health activities projectized. \\
\hline Master SAR & $\begin{array}{l}\text { IA1301, 1A1401, } \\
\text { IA1601, 1A3302, } \\
\text { IA3202, 1A3402 }\end{array}$ & 1A1A04 & $\begin{array}{l}\text { SAR accounts previously spread across many } \\
\text { cost accounts in FY96. Consolidated in FY97 } \\
\text { for activity management and ABC } \\
\text { methodology. }\end{array}$ \\
\hline DOE Walk-in Work & 1A1A0107 & $1 \mathrm{~A} 1 \mathrm{~A} 06$ & $\begin{array}{l}\text { In FY96, activity was a work package. In } \\
\text { FY97, activity was made a unique cost account. }\end{array}$ \\
\hline Patrol & overhead & 1A1M04 & $\begin{array}{l}\text { Patrol costs allocated in the SWS overhead pool } \\
\text { in FY96. In FY } 97 \text {, patrol costs will be } \\
\text { allocated as a direct charge to the programs. }\end{array}$ \\
\hline
\end{tabular}




\begin{tabular}{|c|c|c|c|}
\hline 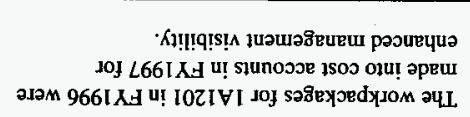 & 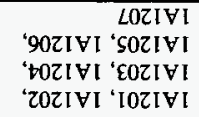 & IOZIVI & גUวunEaII $M W$ \\
\hline 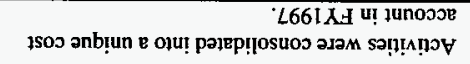 & LOVIVI & IOVIVI 'I06IVI & 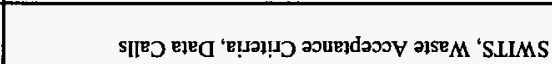 \\
\hline 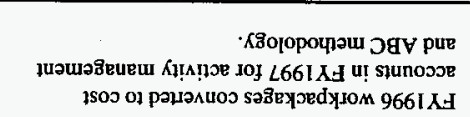 & 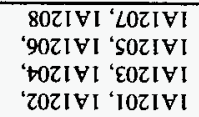 & IOZIVI & 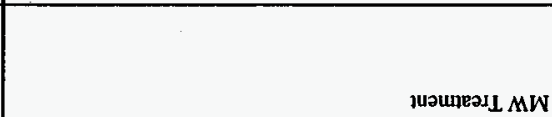 \\
\hline 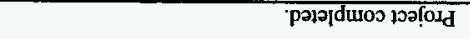 & рәэәра & I-00zZ SAV & $100 ! 0 \mathrm{~d} \mathrm{ZI} \mathrm{I}^{-M}$ \\
\hline 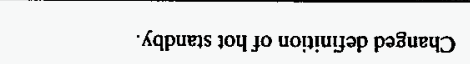 & zoદEVI 'zozદVI & 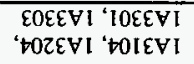 & 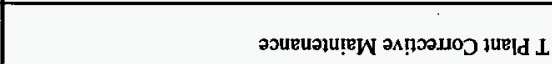 \\
\hline 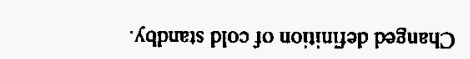 & IOEEVI & $\begin{array}{l}\text { Z0ZEVI 'EOEEVI } \\
\text { 'ZOEEVI '†OIEVI }\end{array}$ & 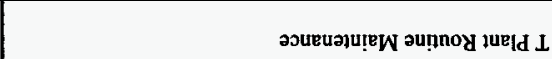 \\
\hline juantuob $(\mathrm{c})$ & 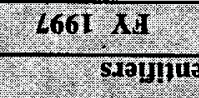 & $\frac{966 \mathrm{~J} \mathrm{NT}}{\mathrm{I} \cdot(\mathrm{I})}$ & 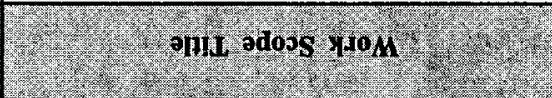 \\
\hline & (1) & $01966 \mathrm{I} \mathrm{XH} \mathrm{-}^{-1}$ & DHC \\
\hline
\end{tabular}


Program: Solid Waste

Baseline Anatysis

(1) Beginning Baseline - FY 1996 MYPP (9/26/95)

(2) FY 1996 Reported Savings (Schedule 2A and 2B) (2.1) Deleted Workscope (includes pending items) (2.2) Efficiencies

(3) FY 1996 Other C/R Activity (Schedule 2A and 2B)

(3.1) Workscope Deferrals

(3.2) Workscope Transfers

(3.3) Workscope Additions - Accelerated

(3.4) Workscope Additions - New (includes pending items)

(4) Prior Year Carryover Workscope

(5) FY 1996 MYPP Net of FY 1996 CR Actions

(6) FY 1997 Baseline Planning Actions (Schedule 3)
6.1 Deleted Workscopo
6.2 Workscope Deferrals
6.3 Workscope Additions - Accelerated
6.4 Workscope Additions - New
6.5 Workscope Transfers
Total 1997 Planning Actions

(7) FY 1997 Approved MWWP Baseline

FY 1997 MYWP GUIDANCE from RL

- Pending resolution of Security and Patrol funding

\begin{tabular}{|c|c|c|c|c|}
\hline 1996 & 1997 & 1998 & 1999 & TOTAL \\
\hline 79.0 & 58.5 & 51.2 & 51.2 & 239.9 \\
\hline$(21.0)$ & (2.0) & & & $(23.0)$ \\
\hline 0.0 & 0.0 & 0.0 & 0.0 & 0.0 \\
\hline
\end{tabular}

\begin{tabular}{|c|c|c|c|c|}
\hline$(0.5)$ & 0.0 & 0.0 & 0.0 & $(0.5)$ \\
\hline 0.0 & 0.0 & 0.0 & 0.0 & 0.0 \\
\hline 0.0 & 0.0 & 0.0 & 0.0 & 0.0 \\
\hline 9.4 & 4.3 & 0.0 & 0.5 & 14.3 \\
\hline 4.4 & 0.0 & 0.0 & 0.0 & 4.4 \\
\hline 71.3 & 60.8 & 51.2 & 51.7 & 235.0 \\
\hline 0.0 & $(11.8)$ & $(7.0)$ & (8.6) & (27.4) \\
\hline 0.0 & 0.0 & 0.0 & 0.0 & 0.0 \\
\hline 0.0 & 1.0 & 0.0 & 0.0 & 1.0 \\
\hline 0.0 & 15.5 & 32.5 & 63.2 & 111.2 \\
\hline 0.0 & 0.0 & 0.0 & 0.0 & 0.0 \\
\hline 0.0 & 4.7 & 25.5 & 54.6 & 84.8 \\
\hline 71.3 & 65.5 & 76.7 & 106.3 & 319.8 \\
\hline & & 76.7 & 106.3 & \\
\hline
\end{tabular}

4.4 5.0

.4) 
Program:

C/R NUMBER SWD-96-053R

SWD-96-062

SWD-96-067

SWD-96-067

SWD-96-068

SWD-96-053R2

SWD-96-XXX

SWD-96-XXX

SWD-96-XXX

SWD-96-XXX
Solld Waste

DESCRIPTION

Approve Work Scope for Project W-485 and W486

Power Swlvel Hook Procurement Funding Change

Support to TWRS Unfunded Work Scope

Support to TWRS Unfunded Work Scope

Transfer Funds from DOE Walk-in Support

\section{SAVINGS TYPE}

Deletion - GPPs

Deletion

Deletion - Expense

Deletion - Expenso

Deletion

Deletion

Sub Total Deletions

Deferral

Deferral

Sub Total Deferrals

Transfer

Transfer

Sub Total Transfers

Addition-Accelerated

Addition-Accelerated

Sub Total Accelerated

\section{FY 1996 Reported Savings}

Approve Work Scope for Project W-485 and W-

C-170 dollars from FY 1995

FY 1996 PBFs

Security patrol

\begin{tabular}{|lrr|r|r|r|}
\hline Addition-New-Small Projects & 0.3 & & & & 0.3 \\
Addition-New & 1.027 & & & & 1.027 \\
Addition-New & 1.658 & & & & 1.658 \\
Addition-New & & 1.015 & & & 1.015 \\
Addition-New & & & & & 0.000 \\
Addition-New & & & & 0.0 \\
\hline Sub Total New & 3.0 & 1.0 & 0.0 & 0.0 & 4.0 \\
\hline
\end{tabular}

Savings Actions

ROF Activity

Discretionary Savings/Underruns

Efficiency

Efficiency

Sub Total Efficiency

\begin{tabular}{|l|l|l|l|l|}
\hline & \multicolumn{3}{c}{} & 0.0 \\
\hline 0.0 & 0.0 & 0.0 & 0.0 & 0.0 \\
\hline
\end{tabular}

C/R Number

Narrative of Malor Savings by Chanoe Request

SWD

SWD-96-053R2 


\author{
(s in Millions)
}

SWD

SWD-96-062

\title{
SWD-96-067 \\ (Expense and Capital
}

L_._.

SWD-96-068

Defer procurement and change funding type from CENRTC FY96 to expense FY97 for power swivel hook.

Delete BCWS from walk-in work in support of RL activities. 
C/R NUMBER SWD-96-002

SWD-96-004R1

SWD-96-005

SWD-96-006

SWD-96-008

SWD-96-010

SWD-96-011

SWD-96-016

SWD-96-019

SWD-96-020

SWD-96-023

SWD-96-033

SWD-96-035

SWD-96-035

SWD-96-008

SWD-96-002 SWD-96-004R1 SWD-96-006

SWD-96-008

SWD-96-010

SWD-96-011

SWD-96-019

\section{DESCRIPTION}

Equipment Storage Bulldings

Changes to Solid Waste Program FY1996

MYPP (Carryover)

improvements in Waste Forecasting

Create FY96 Womens's Change Traller

Transfer Funds to the Asbestos Abatement Portion of W-147

Radloactive Mixed Waste Characterization Project

New Railcar Mobilization

616 Facility Standby Mode

CENRTC Closures and Funding Reallocation ML Feo Elimination

Closeout and Transfer of WRAP 2A CENRTC Funds

Redirection of FY96 Fo for New Workscope

Solid Waste Program Rebaseline Update

Solid Waste Program Rebaseline Update

Transfer Funds to the Asbestos Abatement Portion of W-147

None

Equipment Storage Buildings

Changes to Solid Waste Program FY1996

Create FY96 Womens's Change Trailer

Transfer Funds to the Asbestos Abatement Portion of W-147

Radioactive Mixed Waste Characterization

New Railcar Mobilization

CENRTC Closures and Funding Reallocation
SAVINGS TYPE

Deletion - CENRTC

Deletion

Deletion

Deletion

Deletion

Deletion - DOE Walk-in

Deietion - DOE Walk-in

Deletion - DOE Walk-in

Deletion

Deletion

Deletion

Deletion

Deletion

Deletton - Expense

Deletion - Capital

Deletion

Sub Total Deletions

Deferral-vent duct study

Deferral

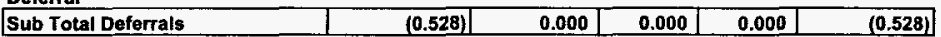

Transfer

\begin{tabular}{|c|c|c|c|c|c|}
\hline Sub Total Transfers & 0.000 & 0.000 & 0.000 & 0.000 & 0.000 \\
\hline Addition-Accelerated & & & & & 0.000 \\
\hline Addition-Acceleratod & & & & & 0.000 \\
\hline Sub Total Accelerated & 0.000 & 0.000 & 0.000 & 0.000 & 0,000 \\
\hline
\end{tabular}

Addition-New - Small Projects

0.280

0.280

Addition-New

0.509

0.509

0.120

0.675

0.023

0.123

0.114

$(4.405)$

0.000

$(0.124)$

(0.120)

(0.147)

$(0.023)$

(0.123)

(0.303)

(0.114)

(1.000)

(0.120)

(2.549)

(7.867)

0.000

Addition-New

0.023

0.123

0.114 
SWD-96-023

SWD-96-033 SWD-96-033

SWD-96-045

SWD-96-057

SWD-96-008
Closeout and Transfer of WRAP 2A CENRTC Funds

Redirection of FY96 Fee for New Workscope Redirection of FY96 Fee for New Workscope PHMC Contract Transition

Site Management Board Mid-Year Review

Transfer Funds to the Asbestos Abatement

Portion of W-147

(\$ in Millions)

Addition-New - Fee Impact
Addition-New - Expense
Addition-New - CENRTC
Addition-New
Addition-New

Addition-New
$0.120 \quad 0.120$

2.326

1.642
3.334

2.326

0.223

0.260

4.976

$0.528 \quad 0.528$

\begin{tabular}{|l|r|r|r|r|r|}
\hline Sub Total New & 6.414 & 3.334 & 0.000 & 0.528 & 10.276 \\
\hline
\end{tabular}

\section{Savings Actions}

ROF Activity

Discretionary Savings/Underruns

Efficlency

Efficiency

Sub Total Efficiency

6.414

0.000

\begin{tabular}{|l|l|l|l|l|l|}
\hline Sub Total Efficiency & 0.000 & 0.000 & 0.000 & 0.000 & 0.000 \\
\hline
\end{tabular}

Narrative of Maior Savings by Change Request

SWD-96-002

CENRTC and Small

BCWS/funds from CENATC to small project. Delete scope associated with equipment purchases in support of new small project scope.

SWD-96-004R1

Reduce FY95 carryover scope from 20 CAAs to 11 CAAs. Reduce FY97 scope of WRAP. Add scope for DOE support and sanitary water line.

SWD-96-005

Reduced scope associated with annual solid waste forecasting.

SWD-96-006
(CENRTC)

Change trailer CENRTC account to complete installation. New scope required by DOH.

SWD-96-008 Defer T Plant vent and duct study and delete DOE walk-in work in support of new asbestos abatement support. 
SWD-96-010

Delete DOE walk-in work in support of PMW characterization project.

SWD-96-011

SWD-96-016 (ML8)

SWD-96-019

SWD-96-020 (ML)

SWD-96-023

(CENRTC)

SWD-96-033

SWD-96-035

(Expense and

CENRTC)

SWD-96-045

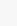

Reduced operations at 616 Facility.

Deletion of fee from ML4, ML5, ML6, and ML8 accounts.

Deletion of FY96 fee to support new workscope items.

New scope in support of transition to new contract.

\section{Delete DOE walk-in work in support of project to provide instruments for T Plant railcar.}

Deletion of scope from 5 CENRTC accounts in support of new scope in 3 separate CENRTC accounts.

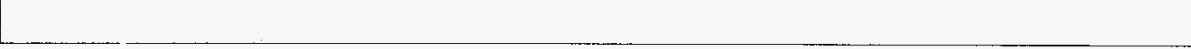

Delete procurement of extruder for WRAP 2A. Add BCWS for impacts caused from C-170 fee payment.

Reductions to FY96 baseline. Deletion of special case waste project $\bar{W}-272$, sodium treatment contract, program management
fee, and W-112 storage facilities. 
( $\$$ In Millions) 
FY 1997 BASELINE PLANNING ACTIONS

Schedule 3

( $\$$ in Millions)

\section{Program: $\quad$ Solid Waste}

FY 1997 Baseline Planning Actions

(6.1) Deleted Workscope

(1) TRUSAF Mortgage Reduction

(2) CENRTC/Small Projects

(3) Rad Area Reduction (revised scope)

(4) Tank Car Certification

(5) Systems Engineering

(6) DOE Walk-in Work

(7) SW Fee

(B) Planned efficiencies

Total

\begin{tabular}{l|l}
1996 & 1997 \\
\hline
\end{tabular}

1998

1999

TOTAL

(6.2) Workscope Deferred

$$
\text { (1) }
$$

Total

\begin{tabular}{|ccccc}
\hline \multicolumn{1}{|c}{0.0} & $(2.1)$ & $(2.9)$ & $(5.0)$ \\
& $(2.5)$ & $(0.8)$ & $(0.9)$ & $(4.2)$ \\
& 0.0 & 0.0 & $(0.4)$ & $(0.4)$ \\
& $(0.2)$ & $(0.2)$ & $(0.2)$ & $(0.6)$ \\
& $(1.0)$ & 0.0 & 0.0 & $(1.0)$ \\
& $(0.9)$ & $(1.0)$ & $(1.1)$ & $(3.0)$ \\
\hline & $(2.0)$ & $(1.6)$ & $(1.6)$ & $(5.2)$ \\
\hline 0.0 & $(5.2)$ & $(1.3)$ & $(1.5)$ & $(8.0)$ \\
\hline
\end{tabular}

(6.3) Workscope Additions - Accelerated
(1) WRAP TRU Startup
(2) Project W-259 Expense Support
Total

\begin{tabular}{|c|c|c|c|c|}
\hline 0.0 & 0.0 & 0.0 & 0.0 & 0.0 \\
\hline 0.0 & 0.0 & 0.0 & 0.0 & 0.0 \\
\hline & 0.6 & 0.0 & 0.0 & 0.6 \\
\hline & 0.4 & 0.0 & 0.0 & 0.4 \\
\hline 0.0 & 1.0 & 0.0 & 0.0 & 1.0 \\
\hline
\end{tabular}

(6.4) Workscope Additions - New
(1) WRAP LLW Processing
(2) WRAP TRU Processing
(3) WRAP NDE/NDA
(4) WRAP Personnel Training
(5) WRAP Facility Maintenance
(6) TRUSAF Transition
(7) CWC High Curie Ops.
(8) MW Trench Storage
(9) Leachate Management
(10) MW Trench Closure Cover Design
(11) Solid Waste EIS
(12) CENRTC/Small Projects
(13) TRU Drum Relocation
(14) Rad Area Reduction
(15) Dirt Cover for Filled Trench
(16) Radcon Path Forward
(17) Facility Evaluation Board

$\begin{array}{ll} & \\ 0.0 & 0.1 \\ 0.0 & 0.4 \\ 0.0 & 0.1 \\ 0.0 & 0.0 \\ 0.1 & 0.0 \\ 0.9 & 1.2 \\ 0.2 & 0.1 \\ 0.6 & 0.7 \\ 0.0 & 0.0 \\ 0.0 & 0.5 \\ 2.4 & 0.1 \\ 0.3 & 2.3 \\ 0.2 & 0.0 \\ 0.3 & 0.0 \\ 0.0 & 0.9 \\ 0.0 & 0.1 \\ 0.1 & 0.0\end{array}$




$$
\begin{aligned}
& \text { Schedule } 3 \\
& \text { ( } \$ \text { in Millions) }
\end{aligned}
$$

(18) Start-up of Storage Bldgs. (W-112)

(19) LLBG Stabilization

(20) PHMC Performance Measures

(21) Vegetation Management

(22) Interim EA for Deep Trench

(23) Master SAR (re-estimate)

(24) Office Facilities Malntenance

(25) CO2 Decon

(26) FHA Upgrade

(27) Sprung Pad Cover

(28) Transfer of Llquid Decon

(29) SNF Removal Activities

(30) Asset Account

(31) Ventilation Upgrade

(32) TRU Treatment Preparation

(33) Additional High-Dose Decon

(34) Additional Low-Dose Decon

(35) Vent Duct Study

(36) Thermal Treatment

(37) MW Stabilization

(38) Macroencapsulation

(39) Direct Disposal

(40) MW Characterization

(41) SmallUnlque Waste

(42) MW Treatment Acceleration

(43) Safety/Health

(44) Patrol

(45) Requirements Management

(46) Systems Engineering

(47) TRU Retrieval (W-113)

(48) TRU Retrieval (W-221)

(49) Caisson Retrieval (W-156)

(51) Burial Ground Closure

(52) Data Management

(53) Misc work scopes

(54) G\&A Increase

Total

(6.5) Workscope Transfers

$$
\text { (1) }
$$

\begin{tabular}{|c|c|c|c|c|}
\hline & 0.2 & 0.0 & 0.0 & 0.2 \\
\hline & 0.0 & 0.3 & 1.5 & 1.8 \\
\hline & 0.1 & 0.1 & 0.1 & 0.3 \\
\hline & 0.2 & 0.2 & 0.2 & 0.6 \\
\hline & 0.1 & 0.0 & 0.0 & 0.1 \\
\hline & 0.0 & 0.7 & 0.7 & 1.4 \\
\hline & 0.3 & 0.3 & 0.3 & 0.9 \\
\hline & 0.7 & 0.7 & 0.7 & 2.1 \\
\hline & 1.1 & 1.2 & 0.0 & 2.3 \\
\hline & 0.2 & 0.0 & 0.0 & 0.2 \\
\hline & 1.1 & 0.8 & 0.0 & 1.9 \\
\hline & 0.2 & 0.4 & 0.3 & 0.9 \\
\hline & 0.0 & 0.2 & 0.2 & 0.4 \\
\hline & 0.0 & 0.0 & 2.0 & 2.0 \\
\hline & 0.0 & 0.0 & 0.2 & 0.2 \\
\hline & 0.6 & 2.4 & 2.3 & 5.3 \\
\hline & 1.2 & 1.8 & 2.5 & 5.5 \\
\hline & 0.0 & 0.0 & 0.5 & 0.5 \\
\hline & 0.6 & 0.3 & 1.2 & 2.1 \\
\hline & 0.0 & 1.0 & 1.0 & 2.0 \\
\hline & 0.0 & 1.3 & 1.7 & 3.0 \\
\hline & 0.5 & 0.7 & 1.5 & 2.7 \\
\hline & 0.5 & 1.7 & 3.3 & 5.5 \\
\hline & 0.0 & 0.7 & 1.6 & 2.3 \\
\hline & 0.0 & 3.9 & 7.7 & 11.6 \\
\hline & 0.1 & 0.0 & 0.0 & 0.1 \\
\hline & 0.7 & 0.7 & 0.7 & 2.1 \\
\hline & 0.3 & 0.3 & 0.2 & 0.8 \\
\hline & 0.0 & 1.0 & 1.0 & 2.0 \\
\hline & 0.0 & 0.8 & 17.9 & 18.7 \\
\hline & 0.0 & 0.7 & 1.8 & 2.5 \\
\hline & 0.0 & 0.2 & 0.5 & 0.7 \\
\hline & 0.0 & 0.5 & 1.9 & 2.4 \\
\hline & 0.0 & 0.8 & 0.9 & 1.7 \\
\hline & 0.9 & 1.5 & 0.4 & 2.8 \\
\hline & 0.8 & 0.8 & 0.8 & 2.4 \\
\hline 0.0 & 15.5 & 32.5 & 63.2 & 111.2 \\
\hline
\end{tabular}$$
\text { Total }
$$

\begin{tabular}{|c|c|c|c|c|}
\hline 0.0 & 0.0 & 0.0 & 0.0 & 0.0 \\
\hline
\end{tabular} 
SOLID WASTE PROGRAM

WBS 1.2.1

FY 1997 Program Plan

WHC-SP-1114. REV 2

\section{Subcontractor Exhibit}

Dollars in Thousands

Project Hanford Breakdown Structure:

Project Hantord Title:
1.2.1

Solid Waste Program

\begin{tabular}{rr}
\multicolumn{2}{c}{ FY 1997} \\
\hline$\$$ & FTES \\
& \\
$\$ 0$ & 0 \\
\hline$\$ 7,452.3$ & 0 \\
\hline$\$ 369.4$ & 0 \\
\hline
\end{tabular}

Service Agreement

Technical Service

Clerical

Construction

Total $\mathbf{0}$

0

\begin{tabular}{cc}
$\$ 6,281.8$ & 0 \\
$\$ 14,035.5$ & 0 \\
\hline
\end{tabular}

\begin{tabular}{|c|c|}
\hline \multicolumn{2}{|c|}{ FY 1998} \\
\hline$\$$ & ETES \\
\hline$\$ 0$ & 0 \\
\hline$\$ 7,209.6$ & 0 \\
\hline$\$ 379.4$ & 0 \\
\hline$\$ 485.1$ & o \\
\hline$\$ 8,074.1$ & 0 \\
\hline
\end{tabular}


繁 


\subsection{Performance Measure: Objectives, Measures and Expectations}

This Fiscal Year 1997 Multi-Year Work Plan (MYWP) has been aligned to the performance measures contained in the Project Hanford Management Contract (PHMC) Best and Final Offer (BAFO) as approved by DOE-RL and aligned to the MDD endpoints as noted in Section 1.0 Technical Baseline.

This MYWP contains supporting goals and objectives (as shown in the Milestone-Description Sheets located in Section 3.3 of this MYWP), measures, and objectives as noted in the attached table for the execution year. These performance measures and the associated metrics can be found in Section 1.2 Project End-Point Targets and in the Milestone Description sheets.

The performance objectives, measures, expectations applicable to the Solid Waste Program are attached with the manager, cost account, and level of milestone. 
SOLID WASTE PROGRAM

WBS 1.2.1

WASTE MANAGEMENT (SOW Section C.3.B)

\begin{tabular}{|c|c|c|c|}
\hline OBJECTIVE & MEASURES & EXPECTATIONS & Rep. Mgr-Cost Account-Milestone \\
\hline \multirow{5}{*}{$\begin{array}{l}\text { WM1 Receive, store, treat, and dispose of } \\
\text { Solid Waste in a safe and environmentally } \\
\text { compliant manner such that capacity can } \\
\text { always satisfy demand, and tisposal costs } \\
\text { are reduced to a level at which they are } \\
\text { preferable to commercially available } \\
\text { altematives. }\end{array}$} & $\begin{array}{l}\text { WM1.1 Conduct safe operations and } \\
\text { maintenance of WRAP } 1 \text { Facility. }\end{array}$ & $\begin{array}{l}\text { WM1.1.1 Initiate operations of Waste } \\
\text { Receiving and Processing (WRAP) Module I } \\
\text { by March 31, } 1997 .\end{array}$ & Norton - A4721 - TPA \\
\hline & $\begin{array}{l}\text { WM1,2 Initiate Project W-259 for 2706-T } \\
\text { (providing double containment). }\end{array}$ & $\begin{array}{l}\text { WM1.2.1 Initiate construction of Project W- } \\
2592706-T \text { secondary containment by } \\
\text { January } 30,1997 \text {. }\end{array}$ & Wright - 3AB166 - RL \\
\hline & \multirow[t]{2}{*}{$\begin{array}{l}\text { WM1.3 Site wide customer waste } \\
\text { management needs analyzed, alternatives to } \\
\text { reduce costs and improve performance } \\
\text { considered and adopted where advantageous. }\end{array}$} & $\begin{array}{l}\text { WM1.3.1 Develop and submit for DOE } \\
\text { approval an integrated site-wide plan to } \\
\text { provide waste management services, } \\
\text { inctuding waste processing, characterization, } \\
\text { and vertication based on design data from } \\
\text { WAAP I, at a cost competitive with } \\
\text { commercial costs by Sept. } 30,1997 \text {. }\end{array}$ & McKenney - A1A1MO1 - RL \\
\hline & & $\begin{array}{l}\text { WM1.3.2 Quality periormance in waste } \\
\text { management functions is verified against } \\
\text { industry benchmarks. }\end{array}$ & McKenney - A1AtMO1 - RL \\
\hline & $\begin{array}{l}\text { WM1.4 Process, package, and ship } \\
\text { decontarninated materiass to the Central } \\
\text { Waste Complex (CWC), bury, or retum to } \\
\text { the customer high activity } \\
\text { waste/contaminated equipment for storage, } \\
\text { disposal, and/or reuse. }\end{array}$ & $\begin{array}{l}\text { WM1.4.1 Provide subject services for less } \\
\text { than } \$ 700 / \text { cubic foot. }\end{array}$ & Wright - 1A3104 - RL \\
\hline
\end{tabular}


SOLID WASTE PROGRAM

WBS 1.2.1

FY1997 Program Plan

WHC-SP-1114. REV. 2

\begin{tabular}{|c|c|c|c|}
\hline \multirow[t]{2}{*}{ OBJECTIVE } & MEASURES & EXPECTATIONS & \multirow{2}{*}{$\begin{aligned} \text { Rep. Mgr-Cost Account-Milestone } \\
\text { Wright - A3204 - RL }\end{aligned}$} \\
\hline & $\begin{array}{l}\text { WM1.5 Process equipment and/or waste for } \\
\text { reuse or disposal as olfered by the } \\
\text { generators. }\end{array}$ & $\begin{array}{l}\text { WM1.5.1 The waste and/or contaminated } \\
\text { equipment shall be processed at a cost less } \\
\text { than } \$ 120 \text { per cubic foot. }\end{array}$ & \\
\hline & \multirow[t]{3}{*}{$\begin{array}{l}\text { WM1.6 Maintain and accept waste into the } \\
\text { low level burial grounds in accordance with: } \\
\text { the authorization basis for the solid waste } \\
\text { burial grounds, the Hanford Site Solid Waste } \\
\text { Acceplance Criteria (HSWAC), the RCRA } \\
\text { Permit Application and supplemental } \\
\text { sulumittals, and } 200 \text { West Area and } 200 \text { East } \\
\text { (when approved) Performance } \\
\text { Assessments. }\end{array}$} & $\begin{array}{l}\text { WM1.6.1 Assessments and audits show } \\
\text { violations of authorization basis, } \\
\text { Performance Assessments, or HSWAC are } \\
\text { less than or equal to } 95 \% \text { of FY96 case rates. }\end{array}$ & \multirow[t]{3}{*}{$\begin{array}{c}\text { Hapke - A1601 - RL } \\
\text { ML4 - WHC }\end{array}$} \\
\hline & & $\begin{array}{l}\text { WM1.6.2 Acceptance of waste as described } \\
\text { by the generator that does not megt the } \\
\text { Hanford Site Solid Waste Acceptance } \\
\text { Criteria is evidenced by audit and } \\
\text { surveillance on waste acceptance } \\
\text { implementation and is less than or equal to } \\
95 \% \text { of FY96 case rates. }\end{array}$ & \\
\hline & & $\begin{array}{l}\text { WM1.6.3 Operations, maintenance, and } \\
\text { waste acceptance are consistent with the } \\
\text { LLBG RCRA permit apptication and } \\
\text { supplemental submittals. Findings of } \\
\text { noncompliance by DOE or Ecology on the } \\
\text { operating record, operations, and procedures } \\
\text { and are less tha/ or equal to } 95 \% \text { of FY } 96 \\
\text { case rates. }\end{array}$ & \\
\hline & $\begin{array}{l}\text { WM1.7 identify technology needs for waste } \\
\text { characterization, treatment, and disposal. }\end{array}$ & $\begin{array}{l}\text { WM1.7.1 Prepare "Mixed Waste } \\
\text { Technology Needs" report yearly to meet the } \\
\text { budget cycle by January 31, } 1997 \text { and } \\
\text { annualy. }\end{array}$ & Josephson - 1201 - RL \\
\hline
\end{tabular}




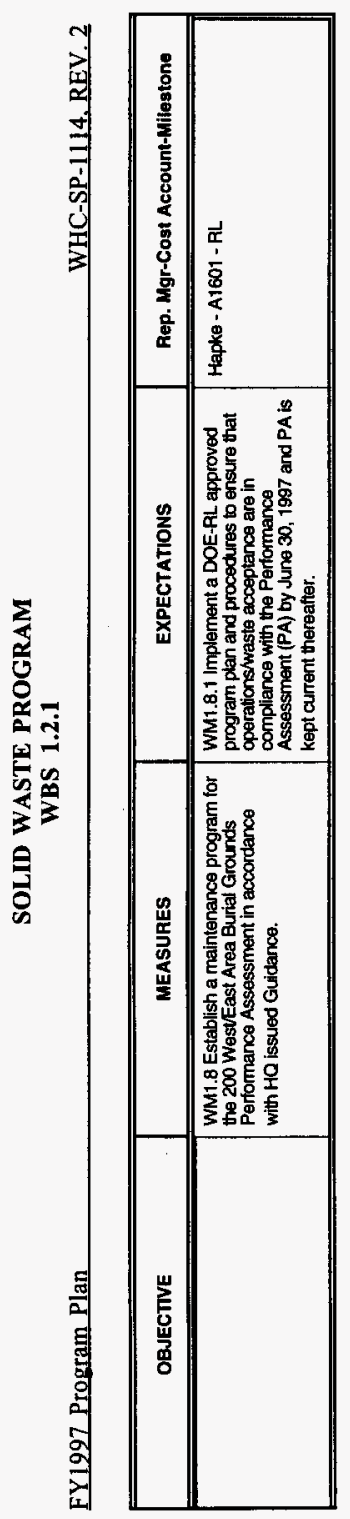




\subsection{Program Performance Baseline Schedule}

The record copies of the Level 4 Performance Baseline Schedules for the Solid Waste Program reside with the appropriate Program Activity Engineer located in MO278/200 West Area. 


\section{SOLID WASTE PROGRAM \\ WBS 1.2.1}

FY 1997 Program Plan

\section{COST BASELINE FOR EXECUTION YEAR BY PROGRAM BY FUND TYPE BY MONTH}

(\$000s)

\begin{tabular}{|c|c|c|c|c|c|c|c|c|c|c|c|c|c|c|c|}
\hline$\frac{\text { RL WBS \# }}{1.2 .1}$ & PROGRAM TITLE & FUND TYPE & OCT & Nov & DEC & JAN & FEB & MAR & APR & MAY & JUN & JUL & AUG & SEP & TOTAL \\
\hline \multirow[t]{8}{*}{1.2 .1} & SOLID WASTE "A" & Expense & $4,508.9$ & $4,785.3$ & $5,239.2$ & $4,714.0$ & $4,757.4$ & 6.158 .9 & $4,959.7$ & $4,853.4$ & $5,826.1$ & $4,142.4$ & $5,536.2$ & $5,675.2$ & $61,156.5$ \\
\hline & & CENRTC & 30.0 & 30.0 & 30.0 & 45.1 & 51.4 & 59.3 & 51.9 & 51.8 & 56.1 & 49.6 & 57.2 & 52.9 & 565.2 \\
\hline & & Line Item & 176.3 & 180.1 & 183.9 & 176.3 & 451.7 & 658.2 & 541.6 & 541.0 & 628.4 & 497.3 & 650.2 & 562.8 & 5.247 .9 \\
\hline & & GPP & 218.4 & 227.2 & 236.1 & 238.1 & 240.4 & 308.3 & 257.6 & 253.6 & 293.5 & 232.6 & 303.6 & 263.0 & $3,072.5$ \\
\hline & & $\begin{array}{l}\text { Total BCWS/PMB (1) } \\
\text { Mgmt Reserve (2) }\end{array}$ & $4,933.5$ & $5,222.7$ & $5,689.3$ & $5,173.4$ & $5,500.9$ & $7,184.7$ & $5,810.8$ & $5,699.7$ & $6,804.1$ & $4,921.9$ & $6,547.2$ & $6,554.0$ & $70,042.1$ \\
\hline & & Line Item Conting (2) & 160.0 & 160.0 & 160.0 & 160.0 & 160.0 & & & 40.0 & 40.0 & 40.0 & 40.0 & 40.0 & $1,000.0$ \\
\hline & & Expected Carryover (3) & & & & 50.0 & 50.0 & 50.0 & 50.0 & 50.0 & 50.0 & 50.0 & 50.0 & 50.0 & 450.0 \\
\hline & & Total & $5,093.5$ & $5,382.7$ & $5,849.3$ & $5,383.4$ & $5,710.9$ & $7,234.7$ & $5,860.8$ & $5,789.7$ & $6,894.1$ & $5,011.9$ & $6,637.2$ & $6,644.0$ & $71,492.1$ \\
\hline 1.2.1.11 & $\begin{array}{l}\text { SOLID WASTE HAZ } \\
\text { ANL ASSESSMENT }\end{array}$ & $\begin{array}{l}\text { Expense } \\
\text { Total BCWS/PMB (1) }\end{array}$ & 19.3 & 20.3 & 21.3 & 19.3 & 19.3 & 26.8 & 21.8 & 20.5 & 24.4 & 18.3 & 25.4 & 21.3 & 258.1 \\
\hline 1.2.1.11 & $\begin{array}{l}\text { SOLID WASTE IML } \\
\text { POOL ASSESSMENT }\end{array}$ & $\begin{array}{l}\text { Expense } \\
\text { Total BCWS/PMB (1) }\end{array}$ & 436.4 & 455.5 & 476.4 & 434.7 & 438.4 & 649.2 & 544.3 & 637.4 & 730.5 & 569.8 & 765.1 & 638.2 & 6.775 .9 \\
\hline
\end{tabular}

\begin{tabular}{|c|c|c|c|c|c|c|c|c|c|c|c|c|c|}
\hline PROGRAM BCWS/PMB & $5,389.2$ & $5,698.5$ & $6,187.0$ & $5,627.4$ & $5,958.6$ & $7,860.8$ & $6,376.9$ & $6,357.7$ & $7,559.0$ & $5,509.9$ & $7,337.8$ & 7.213 .5 & $77,076.2$ \\
\hline$\overline{\text { PROGRAI }}$ & $5,549.2$ & $5,858.5$ & $6,347,0$ & $5,837,4$ & $6,168.6$ & $7,910.8$ & 6.426 .9 & 6.447 .7 & $7,649.0$ & $5,599.9$ & 7.427 .8 & $7,303.5$ & $78,526.2$ \\
\hline
\end{tabular}

(1) Budgeted Cost Of Work Scheduled (BCWS) Equals Performance Measurement Baseline (PMB).

(2) Management Reserve Held By RL And Line Item Contingency Held By RL.

(3) Includes Expected Expense Carryover Requested By Formal Change Control In FY1997; SWD-96-081 pending. 
SOLID WASTE PROGRAM

WBS 1.2.1

FY 1997 Program Plan

COST BASELINE FOR EXECUTION YEAR BY PROGRAM BY COST ELEMENT BY MONTH

WHC-SP-1114, REV. 2

Page 1 of 2

(\$000s)

\begin{tabular}{|c|c|c|c|c|c|c|c|c|c|c|c|c|c|c|c|}
\hline RL WBS \# & PROGRAM TITLE & COST ELEMENT & OCT & NOV & DEC & JAN & FEB & MAR & APR & MAY & JUN & JUL & AUG & SEP & TOTAL \\
\hline \multirow[t]{9}{*}{1.2 .1} & SOLID WASTE "A" & o Salaries \& Cont of Serv & $3,332.1$ & $3,494,0$ & 3.772 .4 & $3,406.1$ & $3,462.4$ & $4,349.0$ & $3,395.2$ & $3,316.2$ & $4,068.9$ & $2,951,3$ & $3,926.2$ & $3,277,0$ & $42,750.8$ \\
\hline & & 1 Material & 146.2 & 159.1 & 178.6 & 173.5 & 177.9 & 239.1 & 158.2 & 152.1 & 188.5 & 144.6 & 196.9 & 158.6 & $2,073.1$ \\
\hline & & 2 Purchased Services & 935.5 & $1,015.4$ & 1.139 .9 & $1,052.7$ & $1,319.6$ & $1,823.2$ & $1,557.5$ & $1,530.2$ & $1,796.9$ & $1,347.8$ & $1,775.2$ & $2,513.4$ & $17,807.4$ \\
\hline & & 3 Charges Other Contr & 37.1 & 39.1 & 41.0 & 37.1 & 37.1 & 48.8 & 35.3 & 35.1 & 42.1 & 31.0 & 42.1 & 35.3 & 461.0 \\
\hline & & 4 Internal Services & 265.9 & 285.6 & 314.3 & 284.1 & 283.8 & 374.1 & 364.6 & 366.8 & 412.6 & 235.5 & 316.3 & 326.3 & $3,829.9$ \\
\hline & & 5 Intemal Charges & 156.5 & 166.0 & 176.3 & 159.5 & 159.5 & 273.1 & 242.0 & 241.2 & 225.5 & 157.0 & 213.6 & 178.7 & $2,348.6$ \\
\hline & & 6 BCS Richland & 22.3 & 23.7 & 25.0 & 22.7 & 22.8 & 27.6 & 18.2 & 18.3 & 21.8 & 18.9 & 27.2 & 22.8 & 271.3 \\
\hline & & 7 Fee & 37.8 & 39.8 & 41.8 & 37.8 & 37.8 & 49.8 & 39.8 & 39.8 & 47.8 & 35.9 & 49.8 & 41.8 & 500.0 \\
\hline & & Salaries are burdened $w$ & $4,933.5$ & $5,222.7$ & $5,689.3$ & $5,173.4$ & $5,500.9$ & 7.184 .7 & $5,810.8$ & $5,699.7$ & $6,804.1$ & $4,921.9$ & $6,547.2$ & $6,554.0$ & $70,042.1$ \\
\hline
\end{tabular}

Cost elements except for fee are burdened with G\&ASWS; Salaries includes org overhead.

\begin{tabular}{|c|c|c|c|c|c|c|c|c|c|c|c|c|c|c|c|}
\hline \multirow[t]{6}{*}{1.2 .1 .11} & SOLID WASTE HAZ & 0 Salaries \& Cont of Serv & 17.8 & 18.7 & 19.7 & 17.8 & 17.8 & 24.9 & 20.2 & 18.9 & 22.5 & 16.9 & 23.4 & 19.7 & 238.2 \\
\hline & ANL ASSESSMENT & 1 Material & 0.9 & 0.9 & 1.0 & 0.9 & 0.9 & 1.1 & 0.9 & 0.9 & 1.1 & 0.8 & 1.1 & 1.0 & 11.5 \\
\hline & (1MDDOP) & 2 Purchased Services & 0.4 & 0.4 & 0.5 & 0.4 & 0.4 & 0.5 & 0.4 & 0.4 & 0.5 & 0.4 & 0.5 & 0.5 & 5.4 \\
\hline & & 4 Internal Services & 0.2 & 0.2 & 0.2 & 0.2 & 0.2 & 0.2 & 0.2 & 0.2 & 0.2 & 0.2 & 0.2 & 0.2 & 2.1 \\
\hline & & 6 BCS Richland & 0.1 & 0.1 & 0.1 & 0.1 & 0.1 & 0.1 & 0.1 & 0.1 & 0.1 & 0.1 & 0.1 & 0.1 & 1.0 \\
\hline & & Total BCW/S/PMB (1) & 19.3 & 20.3 & 21.3 & 19.3 & 19.3 & 26.8 & 21.8 & 20.5 & 24.4 & 18.3 & 25.4 & 21.3 & 258.1 \\
\hline
\end{tabular}

(1) Budgeted Cost Of Work Scheduled (BCWS) Equals Performance Measurement Baseline (PMB) And Is Consistent With BCWS/PMB (Exhibit 3).

Above Amounts Do NOT Include Management Reserve Held By RL, Line Item Contingency Held By RL, Or Expected Expense Carryover Requested By Formal Change Control In FY1997. 


\section{COST BASELINE FOR EXECUTION YEAR BY PROGRAM BY COST ELEMENT BY MONTH \\ Page 2 of 2}

(\$000s)

\begin{tabular}{|c|c|c|c|c|c|c|c|c|c|c|c|c|c|c|c|}
\hline RL WBS \# & PROGRAM TITLE & COST ELEMENT & OCT & NOV & DEC & $\mathbf{J A N}$ & FEB & MAR & APR & MAY & JUN & JUL & AUG & SEP & TOTAL \\
\hline \multirow[t]{8}{*}{ 1.2.1.11 } & SOLID WASTE $1 M L$ & o Salaries \& Cont of Serv & 306.2 & 320.3 & 332.6 & 304.3 & 306.3 & 443.0 & 374.3 & 385.3 & 451.1 & 348.0 & 455.8 & 378.4 & $4,405.6$ \\
\hline & POOL ASSESSMENT & 1 Material & 20.9 & 22.0 & 23.1 & 20.9 & 20.9 & 27.5 & 22.0 & 22.0 & 29.3 & 22.4 & 31.2 & 26.2 & 288.3 \\
\hline & & 2 Purchased Services & 65.2 & 65.9 & 68.5 & 62.0 & 62.0 & 81.8 & 65.5 & 148.7 & 196.0 & 147.0 & 204.2 & 171.5 & $1,338.3$ \\
\hline & & 3 Charges Other Contr & 20.8 & 22.7 & 26.3 & 24.7 & 26.4 & 42.6 & 34.1 & 26.0 & 8.6 & 21.3 & 30.8 & 25.9 & 310.0 \\
\hline & & 4 Internal Services & 22.7 & 23.9 & 25.1 & 22.7 & 22.7 & 29.9 & 24.0 & 31.3 & 39.6 & 29.8 & 41.4 & 34.8 & 347.9 \\
\hline & & 5 Internal Charges & & & & & & 23.8 & 23.9 & 23.9 & 5.8 & 0.9 & 1.3 & 1.1 & 80.8 \\
\hline & & 6 BCS Richland & 0.6 & 0.7 & 0.8 & 0.1 & 0.1 & 0.6 & 0.5 & 0.1 & 0.1 & 0.3 & 0.5 & 0.4 & 5.0 \\
\hline & & Total BCWS/PMB (1) & 436.4 & 455.5 & 476.4 & 434.7 & 438.4 & 649.2 & 544.3 & 637.4 & 730.5 & 569.8 & 765.1 & 638.2 & $6,775.9$ \\
\hline
\end{tabular}

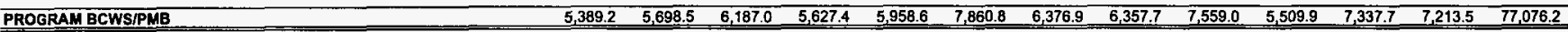

(1) Budgeted Cost Or Work Scheduled (BCWS) Equals Performance Measurement Baseline (PMB) And Is Consistent With BCWS/PMB (Exhibit 3)

Above Amounts Do NOT Include Management Reserve Held By RL, Line Item Contingency Heid By RL, Or Expected Expense Carryover Requested By Formal Change Control In FY1997. 
WBS 1.2 .1

FY 1997 Program Plan

\section{COST BASELINE BY YEAR BY ADS}

Page 1 of 3

(\$000s)
WHC-SP-1114, REV. 2

FY1997 FY1998 FY1999 FY2000 FY2001 FY2002 FY2003 FY2004 FY2005 FY2006 TOTAL

\begin{tabular}{|c|c|c|c|c|c|c|c|c|c|c|}
\hline $30,784.9$ & 444639 & 537215 & & & & & & & & \\
\hline 2971 & & & & $10,3<1,0$ & $94,413.4$ & $99,786.7$ & $93,529.0$ & $104,207.4$ & $88,858.7$ & $756,594.6$ \\
\hline 297.1 & $1,426.2$ & $1,218.6$ & $1,223.2$ & $1,258.3$ & $1,290.6$ & $1,325.0$ & $1,360.7$ & $1,397.6$ & 1.435 .3 & $12,230.6$ \\
\hline 140.7 & 600.0 & $1,500.0$ & $1,539.2$ & $1,579.4$ & $1,621.2$ & $1,663.0$ & $1,706.5$ & $1,751.3$ & $1,790.0$ & $13,891.3$ \\
\hline $31,222.7$ & 46.490 .1 & $56,440.1$ & 64.263 .1 & $78,163.2$ & $87,325.2$ & $102,774.6$ & $96,597.2$ & $107,356.3$ & $102,084.0$ & $782,716.5$ \\
\hline 450.0 & & & & & & & & & & 450.0 \\
\hline $31,672.7$ & $46,490.1$ & $56,440.1$ & $64,263.1$ & $78,163.2$ & $97,325.2$ & 102.774 .6 & $96,597.2$ & $107,356.3$ & $102,084.0$ & $783,168.5$ \\
\hline
\end{tabular}

$10,658.7$

48.0

$10,658.7$

48.0

CENRTC

GPP

Total ECWS/PMB (1)

Mgint Reserve (2)

Line llem Contingency (2)

Expected Carryover (3)

Total

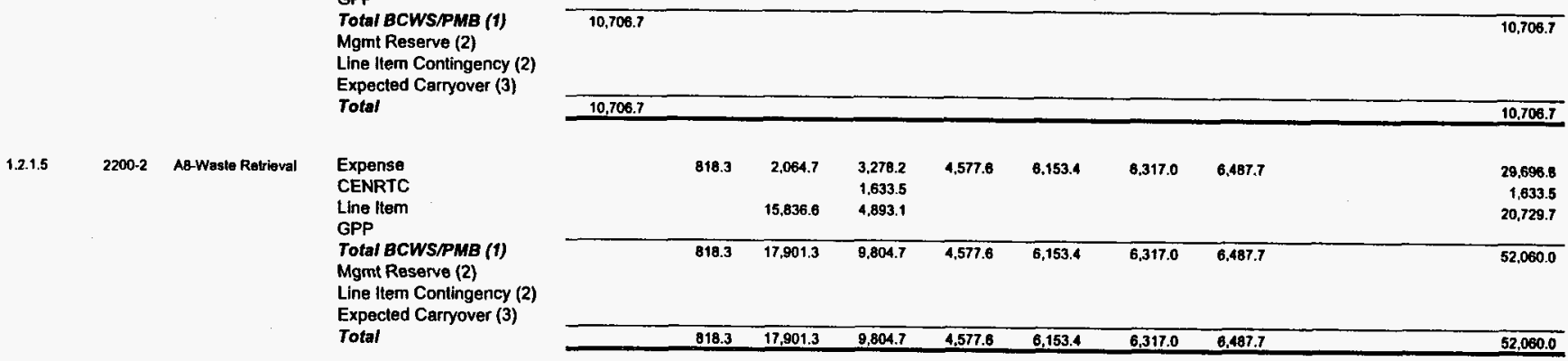


SOLID WASTE PROGRAM

WBS 1.2.1

COST BASELINE BY YEAR BY ADS

Page 2 of 3

(\$000s)
FY 1997 Program Plan

\section{WHC-SP-1114, REV. 2}

\begin{tabular}{|c|c|c|c|c|c|c|c|c|c|c|c|c|c|c|}
\hline RL WBS \# & ADS \# & TITLE & FUND TYPE & FY1997 & FY4998 & FY1999 & FY2000 & FY2001 & FY2002 & FY2003 & FY2004 & FY2005 & FY2006 & TOTAL \\
\hline \multirow[t]{7}{*}{1.2 .1 .6} & $2250-0$ & A9-New Facllity Planning & Expense & & $9,422.7$ & $4,158.0$ & $2,223.5$ & $2,876.9$ & 3.734 .1 & $2,848.6$ & $2,910.7$ & 4.125 .7 & $6,654.9$ & $30,755.1$ \\
\hline & & & CENRTC & & & & & 969.2 & $1,055.6$ & 993.0 & 1.019 .9 & 1.047 .4 & $2,550.1$ & $8,435.1$ \\
\hline & & & Line ltem & & & & 4.581 .3 & $13,028.9$ & $26,927.7$ & $22,726.3$ & $27,945.7$ & 21.523 .7 & 22.104 .5 & 138,838.1 \\
\hline & & & $\begin{array}{l}\text { GPP } \\
\text { Total BCWS/PMB (1) }\end{array}$ & & 1.4227 & & & & & & & & & $\frac{-}{178029}$ \\
\hline & & & $\begin{array}{l}\text { Torar BCW } \\
\text { Mgmt Reserve (2) }\end{array}$ & & $1,422.7$ & 4.158 .0 & $6,804.8$ & $16,875.0$ & $32,517.4$ & $28,367.9$ & $31,876.2$ & $26,696.8$ & $31,309.5$ & $178,028.3$ \\
\hline & & & $\begin{array}{l}\text { Line Item Contingency (2) } \\
\text { Expected Carryover (3) }\end{array}$ & & & & & & & & & & & \\
\hline & & & Total & & 1.422 .7 & $4,158.0$ & 6.804 .8 & 16.875 .0 & $32,517.4$ & $26,367.9$ & $31,876.2$ & $26,696.8$ & $31,309.5$ & $178,028,3$ \\
\hline \multirow[t]{6}{*}{ 1.2.1.7 } & $2320-0$ & A3.Wasle \& Decon Sivc & Expense & $19,022.1$ & $21,750.0$ & 22.426 .8 & 23.004 .7 & 22.422 .8 & $24,576.2$ & $25,089.1$ & $25,563.0$ & 26.445 .7 & $27,079.4$ & $237,379.9$ \\
\hline & & & CENRTC & 220.0 & 796.0 & 817.4 & 839.5 & 862.2 & 885.8 & 909.4 & 933.9 & 959.2 & 885.1 & 8.208 .4 \\
\hline & & & Line llem & & & & $7,920.4$ & $10,853.3$ & & & & & & 18.773 .7 \\
\hline & & & GPP & $2,931.8$ & $1,900.2$ & 1.957 .5 & 152.8 & & & & & & & $6,942.3$ \\
\hline & & & $\begin{array}{l}\text { Total BCWS/PMB (t) } \\
\text { Mgmt Reserve (2) } \\
\text { Line Itern Contingency (2) } \\
\text { Expected Carryover (3) }\end{array}$ & $22,173.9$ & $24,448.1$ & $25,201,8$ & $31,917.5$ & $34,138.2$ & $25,462.0$ & 25.998 .5 & 26.496 .9 & $27,404.9$ & $28,064.5$ & $271,304,3$ \\
\hline & & & Total & 22.173 .9 & $24,446.1$ & $25,201.8$ & $31,917,5$ & $34,138.2$ & $25,462.0$ & $25,998.5$ & $26,496.8$ & $27,404.9$ & $28,064.5$ & $271,304.3$ \\
\hline \multirow[t]{5}{*}{1.2 .1 .8} & $2320-2$ & AB-T Plant 2nd Cont & $\begin{array}{l}\text { Expense } \\
\text { CENRTC }\end{array}$ & 690.6 & 785.8 & 717.2 & & & & & & & & $2,193.8$ \\
\hline & & & $\begin{array}{l}\text { Line Item } \\
\text { GPP }\end{array}$ & $4,099.9$ & $2,700.0$ & $1,899.8$ & & & & & & & & $8,699.5$ \\
\hline & & & $\begin{array}{l}\text { Tolal BCWS/PME (1) } \\
\text { Mgmt Reserve (2) }\end{array}$ & $4,790.8$ & $3,485.7$ & $2,616.8$ & & & & & & & & $10,893.3$ \\
\hline & & & $\begin{array}{l}\text { Line Item Contingency (2) } \\
\text { Expected Carryover (3) }\end{array}$ & 200.0 & & & & & & & & & & 200.0 \\
\hline & & & Tolal & $4,990.8$ & $3,485.7$ & $2,616.8$ & & & & & & & & $11,093.3$ \\
\hline
\end{tabular}


SOLID WASTE PROGRAM

WBS 1.2.1

FY 1997 Program Plan

COST BASELINE BY YEAR BY ADS

Page 3 of 3
(5000s)

WHC-SP-1114, REV.2

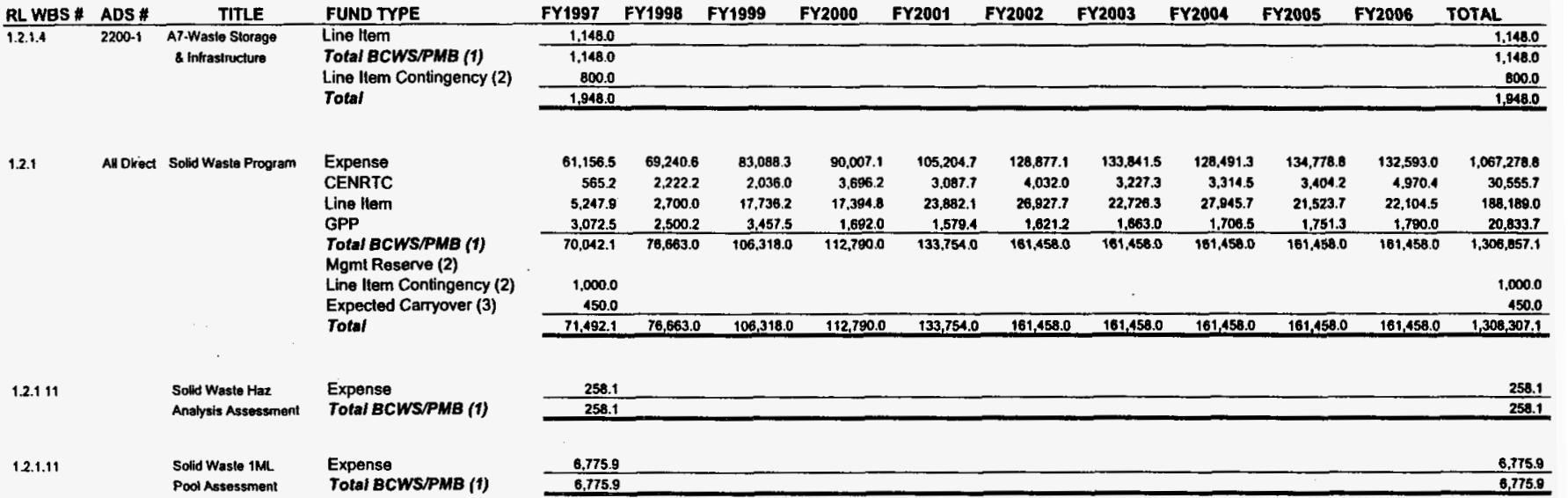

\begin{tabular}{|c|c|c|c|c|c|c|c|c|c|c|c|}
\hline PROGRAM BCWS/PMB & 77.076 .2 & 76.663 .0 & $106,318.0$ & $112,790.0$ & $133,754.0$ & $161,458.0$ & $161,458,0$ & $161,458.0$ & $181,458.0$ & $161,458.0$ & $1,313,891.2$ \\
\hline PROGRAM TOTAL & $76,526.2$ & $76,663,0$ & 106.318 .0 & $112,700.0$ & $133,754.0$ & $161,458,0$ & $161,458.0$ & $161,458.0$ & $161,458.0$ & $161,458.0$ & $1,315,341.2$ \\
\hline
\end{tabular}

(1) Budgeted Cost of Work Scheduled (BCWS) Equals Performance Measurement Baseline (PMB).

(2) Management Reservo And Line ltem Contingency Hald By RL.

(3) Includes Expected Expense Carryover Requested By Formal Change Control in FY 1997 


\section{DISTRIBUTION}

Number

of Copies

MSIN

\section{QFFSITE}

2 United States Department of Energy. Headquarters

Rob Martinez, EM-361

12800 Middlebrook Road

Germantown, MD 20874

1 Washington State Department of Ecology

Moses Jaraysi

$1315 \mathrm{~W}$. 4th Ave.

Kennewick, WA 99336

1 United States Environmental Protection Agency

D. Sherwood

712 Swift Boulevard, MSIN B5-01

Richland, WA 99352

\section{ONSITE}

6 U.S. Department of Energy.

Richland Field Office

M. S. French

S7-55

A. K. Crowell

S7-55

K. D. Bazzell

S7-55

R. F. Guercia

S7-55

T. K. Teynor

S7-55

S. L. Jones

2 Rust Federal Services-Richland

D. B. Van Leuven

H5-32

R. T. Wilde

H5-32

Distr.-1 
27 Westinghouse Hanford Company

N. J. Capron

T4-05

F. T. Green

H6-29

M. R. Ibatuan

T4-52

P. H. Jacobsen (10)

T3-01

M. E. Kurts

T3-01

M. J. La Barge

T3-28

J. J. Lang

T3-01

E. F. Mares

T3-01

D. E. McKenney (3)

T3-01

L. L. Powers

B3-63

J. G. Riddelle

R. R. Slaybaugh (2)

T3-01

Document Processing Center

T3-01

Central Files

A3-94

Unclassified Document Control

A3-88

A4-65

Distr.-2 